\title{
A Microprocessor Based Weighing G Feed Control System
}

Thesis submitted for the Degree of Master of Science

(Engineering)

By: J.H. POTGIETER B.Sc. (Eng:)

Department of Electrical Engineering,

University of Cape Town.

\section{U C T/1975}

The copyright of this thesis is held by the University of -..pe Toun.

Reproduction of tho whole or any part may be made for study purposes only, and not for publication. 
The copyright of this thesis vests in the author. No quotation from it or information derived from it is to be published without full acknowledgement of the source. The thesis is to be used for private study or noncommercial research purposes only.

Published by the University of Cape Town (UCT) in terms of the non-exclusive license granted to UCT by the author. 
The following abstract was submitted for the SECOND IFAC SYMPOSIUM ON AUTOMATION IN MINING, MINERAL AND METAL PROCESSING. held at Johannesburg, during April, 1976.

\section{ABSTRACT, \\ A MICRO-PROCESSOR BASED WEIGHING AND FEED CONTROL SYSTEM.}

By J. H. Potgieter B.Sc. (Eng.) and M.G. Rodd. Pr. Eng. . B. Sc. (Eng. ), M. Sc. (Eng.)

This paper describes the development of a micro-processor based computer control system for the raw material weighing and feed system of a submerged arc ferro-alloy furnace.

The system is designed to replace the more conventional semi-automatic analogue controller at present in use in many installations. It may be used either as a stand-alone unit or as an out-station in a distributed control computer-based system. In the latter, the micro-processor unit forms an intelligent peripheral to the central control computer.

The control unit consists of an 8-bit micro-processor with 8 kilowords of storage. It can control, at present. 3 weighing hoppers fed from 12 raw material storage bins. It responds to service requests from any of the furnace feed hoppers by setting up the necessary conveyor belt and shuttle routing and then feeding the contents of the weighing hoppers onto the transport system.

In addition to the above functions, it is capable of compensating for weighing hopper overshoot, tarring errors, moisture content of raw materials, and can effect local changes in the raw material composition being fed to the furnace. Set points would typically be entered manually via an operator's console. but may be changed at any time, either by the operator or from information supplied by a central computer.

The system is equipped with a teleprinter. This may be used to make setpoint alterations, print-out inventories at selected times, record any changes made, and so on. Any in formation typed out is accompanied by a time-of-day record.

The system described was developed in the Department of Electrical Enginee ring, University of Cape Town, for the National Institute of Metallurgy.

29th. September, 1975.

It has now been confirmed that "A Microprocessor Based Weighing and Feed Control System" has been accepted as a paper to be presented at the above Conference. 


\section{ACKNOWLEDGMENTS,}

(1) To Mike Rodd for his invaluable assistance and guidance during the past two years.

(2) To the National Institute for Metallurgy for their financial and technical support.

(3) To the staff of the Electrical Engineering Department of the University of Cape Town, for their assistance.

(4) To Prof. Ken MacGregor for his advice on the development of software systems.

(5) To Mike and Sue Rodd, Joy Davis and Iona Shand for their endless patience in the scrutiny of and/or typing of the script.

(6) To my brother, Frikkie, for the photography.

(7) To Philip Titus for his help with the endless electrical connections which had to be made.

(8) And, Lastly, to my wife, Reinett, for her patient support during the last two years. 


\section{CONTENTS.}

SECTION 1: OVERVIEW.

Chapter 1 - Arc Furnaces and the need to improve their control.

1.0 - Introduction

1.1 - Description of the Witbank System

1.2 - Disadvantages of the Conventional System

1.3 - The need to improve

Chapter 2 - Approaches to Improve the Control of the Weighing System

2.1 - Choice of a Control System

2.2 - The Need for a Digital Computer 10

2.3 - Why a Micro-Computer ? 11

2.4 - The Micro-Computer based System 12

Chapter 3 - The Microprocessor

3.1 - History

3.2 - Architecture

3.3 - Applications

Chapter ${ }_{\lambda}^{4}-$ Proposed System

4.1 - Weighing Hoppers and their Feeders 24

4.2 - The Holding Bins and their Feeders 25

4.3 - The Furnace Hoppers $\quad 25$

4.4 - The Routing 25 .

4.5 - Moisture Compensation 25

4.6 - Taring Error $\quad 26$

4.7 - Overshoot Error : 26

4.8 - Shut-Down System after each Cycle ? 26 :

4.9 - External Communication 27 :

4.10 - Interference with Existing Conventional System 27 .

SECTION 2 : SYSTEM HARDWARE. $\quad 29$

Chapter 5 - An Introduction to the Hardware $\quad 30$

5.1 - General $\quad 30$

5.2 - Integrating the Prototype with the Real Process

5.3 - Selection of Electronic Components 32

5.4 - The Intel 8008 Microprocessor . 33

5.5 - A Functional Description of the Processor

Chapter 6 - The Processor $\quad \cdots 2$

6.1 - The 8008 and its Control Circuits 42 
Contents - Continued.

6.2 - The Front Panel and its Associated Circuitry 45

6.3 - The Memory 48

6.4 - Input-Output Device Address Decoding $\quad 49$

6.5 - Interfacing of a Minicomputer $\quad: \quad 55$

6.6.- The Direct Memory Access Loader and Display 59

6.7 - External Hardware Registers $\quad 61$

6.8 - The Interrupt Controller $\quad 61$

6.9 - Interface to a Teletype 65

6.10-The Timer and Communication with the Process 65

$\begin{array}{ll}\text { Chapter } 7 \text { - Power and Power Failure } & 70\end{array}$

Chapter 8 - The Process Interface $\quad 73$

6.1 - Introduction 73

6.2 - The Interface $\quad 73$

8.3 - Process Initiated Communication $\quad 75$

8.4 - System Start and Restart $\quad 75$

Chapter 9 - The Simulator $\quad 78$

9.1 - Introduction $\quad 78$

9.2 - The Weighing System Simulator $\quad 78$

9.3 - Simulating the Transport System $\quad 80$

9.4 - Furnace Hopper Level Indicators $\quad 81$

9.5 - Moisture Content $\quad 81$

SECTION 3: THE SYSTEM SOFTWARE

Chapter 10 - Software Philosophy 83

10.1 - Objectives of Software for the Prototype System : 83

10.2 - System Flow Diagram 84

10.3 - Software Notation 88

Chapter 11 - The Utility Routines 91

11.1 - Double Word Addition $\quad 91$

11.2 - Increment the Memory Registers $\quad 91$

11.3 - Decrement the Memory Registers $\quad 92$

11.4 - Obtain 2's Complement $\quad 92$

11.5 - Double Word Subtraction . 92

11.6 - Intezer Division $\quad 93$

11.7 - Integer Multiplication $\quad 94$

11.8 - Number Output to the Teletype $\quad 94$

11.9 - Interrupts when Outputting to a Teletype 95 


\section{Contents - Continued}

$11.10-$ Number Input from the Teletype $\quad 96$

11.11 - Interrupts when Inputting from the Teletype $\quad 97$

11.12 - BCD to Binary Conversion $\quad 98$

11.13 - Sub-Routine Calc. $\quad 98$

11.14 - Binary to BCD Conversion $\quad 98$

11.15 - To Output Alpha Numeric Data via Teletype $\quad 99$

11.16 - Control Input via the Teletype $\quad 99$

$\begin{array}{ll}11.17 \text { - Record Time of Day } & 100\end{array}$

$\begin{array}{ll}11.18 \text { - Print Time of Day } & 101 .\end{array}$

$\begin{array}{ll}11.19 \text { - To Time a Short Delay } & 101\end{array}$

Chapter 12 - The Control Programs 103

12.1 - Loading the Weighing Hoppers 103

12.2 - To Perform Error Corrections on the Selected Setpoints 109

12.3 - To Address the Current Setpoint 111

12.4 - To Determine the General Setpoints 113

12.5 - To Print the Setpoints onto the Teletype 114

12.6 - To Change the Control Parameters from the Teletype. 117

12.7 - To Produce a Shift Report 121

12.8 - To Update the Inventory Record 123

12.9 - To Determine whether a Furnace Hopper requires attention 126

12.10 - To Select the next Furnace Hopper to be Serviced 126

12.11 - To Select which Furnace Hopper is to be Serviced 126

12.12 - To Load a Special Recipe for a Selected Furnace Hopper 131

12.13 - To coordinate all the Different Routines into a Control Program 132

SECTION 4 - FROM PROTOTYPE TO PROCESS

Chapter 13 - Equipment Failure $\quad 134$

13.1 - Introduction 134

13.2 - Processor Failure . . . 134

13.3 - Process Failure $\quad 135$

13.4 - Corrective Measures $\quad 136$

Chapter 14 - Prototype Evaluation $\quad 138$

$\begin{array}{ll}14.1 \text { - Introduction } & 138\end{array}$

14.2 - Hardware Evaluation $\quad 138$

$\begin{array}{ll}14.3 \text { - Software Evaluation } & 140\end{array}$

$\begin{array}{ll}14.4 \text { - System Evaluation } & 141\end{array}$

14.5 - Conclusion 143 
Contents - Continued.

BIBLIOGRAPHY $\quad 144$

$\begin{array}{ll}\text { APPENDICES } & 146\end{array}$

APPENDIX 1 - MICROPROCESSOR SCORECARD A1

APPENDIX 2 - 8008 DATA $\quad$ A2

APPENDIX 3 - 2102 DATA $\quad$ A23

APPENDIX 4 - 8212 DATA $\quad$ A26

APPENDIX 5 - WITBANK SYSTEM PARAMETERS $\quad$ A27

APPENDIX 6 - SOFTWARE LISTING 
1

SECTION 1.

OVERVIEW 


\section{CHAPTER 1}

\section{ARC FURNACES AND THE NEED TO} IMPROVE THEIR CONTROL

"The weighing and charging system represents a most important group of control variables" - Elken Corpany, Norway

\subsection{INTRODUCTION}

During the last few years, large submerged-arc furnaces have been commissioned in the South African ferro-alloy industry. It has been found that such furnaces, although more economical in operation than earlier, smaller furnaces, suffer considerably from problems relating to their design, operation, choice of raw materials, and composition of feed mixture. These problems result in inefficient operation of the furnaces.

In view of the economic importance of the ferro-alloy industry to South Africa, the National Institute of Metallurgy embarked on an investigation into the use of computers for the control of large, submergedarc furnaces. $\{2,3,4,5\}$

The furnace chosen for the investigation was a $48 \mathrm{MNA}$ ferro-chromium furnace situated at Witbank.

As a result of this investigation it was decided to investigate the defects of, and improvements to, the existing conventional, semi-automatic analogue batch-weighing and feed-mix transport and furnace-charging systems used in these types of furnaces. As a specific exarple, the one used at Witbank (the Witbank system) was to be considered. This system is used as a typical example throughout this thesis.

\subsection{DESCRIPTION OF THE WITBANK SYSTEM}

Figure 1.1 shows a mimic panel of the raw-material batch-weighing and transport system.

$\mathrm{H} 1$ to $\mathrm{HLO}$ are a battery of holding bins containing the different raw materials, such as crushed metal ores, and suitable reductants, such as coke, fluxes, and so on. 


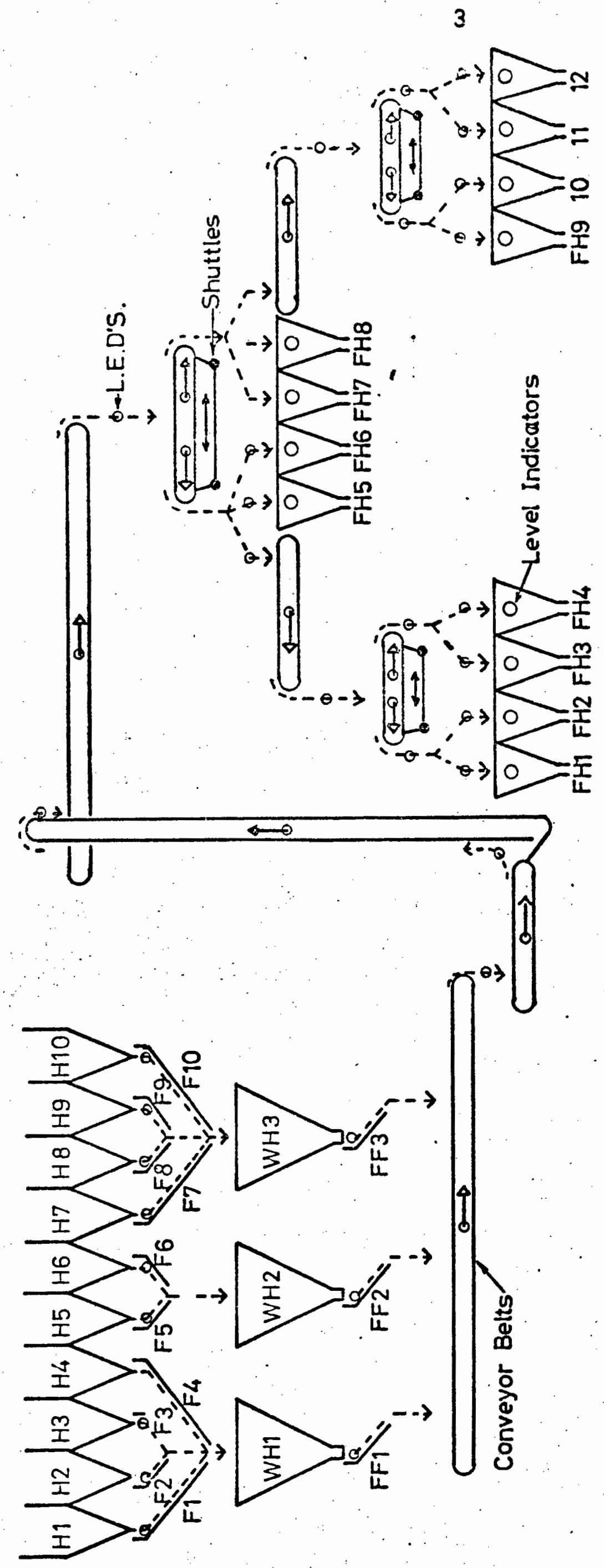

息 
(Chapter 1 - continued)

From these bins the raw materials are fed into one of the three weighing hoppers (WHI - WH3) suspended on load cells. The actual feeding operation is achieved by means of two-speed vibration feeders (F1 - F10). The feeders operate in a "coarse mode" until the weight in a weighing hopper is within $90 \%$ of a desired setpoint (a value at which a particular feeder must be stopped ). The system then changes over to a "fine mode" of feeding until the setpoint is reached. When the weighing hoppers have been filled with the desired proportions of raw materials they are emptied onto the transport system via three additional feeders (FFI - FF3). These feeders are adjusted so that the materials from each of the weighing hoppers form three equidistant layers on top of one another on the conveyor belts. This procedure is to assist in the mixing of the materials. The feed-mix is then transported by means of a system of conveyor belts and shuttles to one of the furnace hoppers (FHI - FHL2), as required. Level indicators on the furnace hoppers determine which hopper is to be serviced. The charge $\mathrm{mix}$ is fed into the furnace by gravity through tubes which lead through the furnace hoppers through the furnace cover. At present, there are four feeding points round each electrode. (Figure 1.2) For safety reasons, and to allow for maintenance and repair shutdown of the charging system, the fumace hoppers are large enough to hold sufficient material to feed for several hours.

\subsection{DISADVANTAGES OF THE CONVENTIONAL SYSTEM}

Before attempting to improve the existing, conventional system, the disadvantages of the system must be outlined.

(a) Uncontrolled and varying overshoots of the weighing hopper input feeders give rise to weighing errors which may have a magnified effect on the weight ratio between the different raw materials. This is caused by 


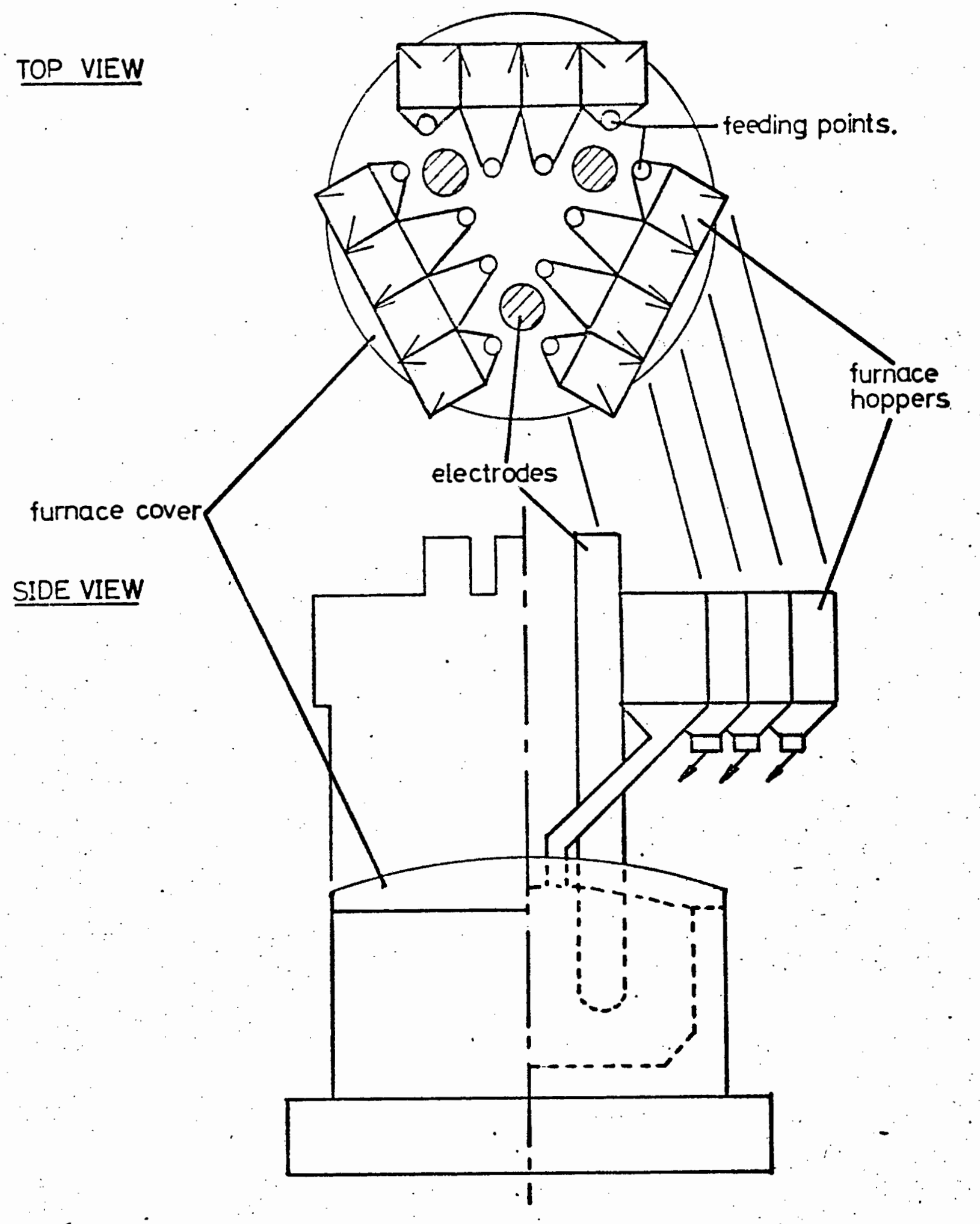

FIGURE 1.2 DIAGRAMMATICAL SKETCH: SHOWING THE RELATIVE POSITIONS OF THE ELECTRODES. FURNACE HOPPERS AND FURNACE FEEDING POINTS IN AN ENCLOSED SUBMERGED ARC FURNACE. 
the successive feeding of batches of several materials into the same hopper and using accumulated setpoints. The meaning of "accumulated setpoints" in this context may be illustrated by the following example: take the few feeders supplying WHI, and assume that the following recipe is required:

$$
\begin{array}{r}
100 \mathrm{~kg} \text { from Hl } \\
50 \mathrm{~kg} \text { from H2 } \\
150 \mathrm{~kg} \text { from H3 } \\
120 \mathrm{~kg} \text { from } \mathrm{H} 4
\end{array}
$$

The accumulated setpoints will then be:

$$
\begin{aligned}
& \text { SP1 }=100 \\
& \text { SP2 }=150 \\
& \text { SP3 }=300 \\
& \text { SP4 }=420
\end{aligned}
$$

The weight ratio error will exist because raw materials of the same composition are normally fed from the same holding bin in the same order. (b) When a weighing hopper is supposed to be empty, the load cell output may not show zero weight of material. This is caused by:

(i) material sticking to the weighing hopper or to its output feeder

(ii) drifts in the load cell amplifiers

(iii) supply voltage variations

The fact that no automatic compensation for this "taring error" exists leads to a considerable percentage error.

(c) "Accumulated setpoints" imply that if the desired amount of any one setpoint is to be changed, alI the succeeding setpoints have to be changed accordingly. This must be done at present by manual adjustments of the setpoint potentiometers.

(d) All the furnace hoppers are treated equally and receive a feed-mix of the same recipe, determined by one set of setpoints. The possibility of introducing changes to the feed-mix recipe locally in the furnace is not provided for. It is often desirable to change, for example, the coke addition to different furnace feeding points . 


\section{(Chapter 1 - continued)}

(e) The setpoint representation by means of potentiometers is unsuitable for automatic setpoint control. This is a disadvantage, not only for sophisticated furnace control, but also for simple applications, such as moisture compensation, ash compensation, and so on.

(f) No provision is made for moisture compensation. As the coke can sometimes contain up to $16 \%$ moisture, this is a serious disadvantage. Similar compensation could be provided for the ash content of the coke. (g) The only automatic aid in determining an inventory of raw materials consumed are electro-mechanical counters for the number of batches fed to the weighing hoppers of each material. This means that the inventory record relies on manual data-logging and calculation.

(h) The whole system operates on a fixed cycle which is inflexible and time-consuming; for example, if the same furnace hopper is to be serviced twice consecutively, all the feeders, conveyors, shuttles, and so on, must be shut down completely before commencing the second cycle. The system's stable state, i.e. waiting for a furnace hopper to become empty, is achieved when the weighing hoppers are fully charged. This $\mathrm{m}$ akes load correction difficult.

(i) There is no provision for external management adjustment of the setpoints. All the adjustments have to be made manually by operators whose reliability may sometimes be questioned. Ideally one would like to be able to make adjustments from a central computer or a data terminal. It would also be desirable to have all adjustments properly logged.

\subsection{THE NEED TO IMPROVE}

As previously stated, the larger arc furnaces suffer from inherent difficulties which result in inefficient operation, but is it worth spending time and money on improving the weighing and charging systems?

Certainly; the Elkern Company in Norway think it well worthwhile: "Proportioning of the feex mix and adjustments of the charge 
(Chapter 1 - continued)

composition - for the furnace as a whole, or locally. are the only control means available besides altering the electrode positions or the transformer voltage. Therefore, it is essential that the weighing system is designed as a practical control tool, so that desired control actions can be effected quickly and accurately, whether they are decided by an operator or by an automatic controller. Secondly, as most of the disturbances causing process variations which require control actions are caused by the raw materials in the first place, an accurate weighing system may help to keep these disturbances as small as possible. Further, as far as the primary disturbances (e.g. varying coke misture content) can be measured, they should be compensated for by a feed-forward control of the weighing setpoints before they reach the furnace. Poor and varying properties of the raw materials are common excuses for difficulties with furnace operation, and it is frequently assumed that better raw materials or expensive pre-treatment only can improve the situation. However, we believe that insufficient accuracy of the weighing system in many cases is more to blame than the raw material quality, and that variations in the raw materials could have been compensated for by a better control system for the weighing and charging." '\{1\}

Local experience suggests that the above is probably accurate. This, along with the need for accurate inventory and information logging, makes the design of a new batch-weighing and raw-material distribution system desirable. 


\section{CHAPTER 2}

APPROACHES TO IMPROVE THE CONTROL

OF THE WEIGHING SYSTEM

In order to improve the control of a submerged arc fumace weighing system, a careful study of the shortcomings of the conventional system must be made. Chapter I revealed the following defects:

(a) No error feedback. No provision is made to ascertain the overshoot or taring errors. The correction of these should be made in succeeding weighing batches.

(b) No attempt is made to determine the effect of the feed-mix recipe on the furnace performance and to make suitable local or universal corrections of the recipe being fed to different zones in the furnace.

(c) No error feedforward. No attempt is made to allow for the possible defects of the raw materials (e.g. the moisture or ash content of the coke).

(d) Lack of accurate inventory and logging information.

(e) Poor provision for external management adjustments.

\subsection{CHOICE OF CONTROL SYSTEM}

A full cycle of the weighing system takes approximately four minutes. There might be delays of hours between the servicing of specific furnace hoppers. If it is hoped to perform any of the feedback functions, the control system will have to store data for at least the duration of these periods.

The latter factor, together with the inventory requirements, implies that some form of data storage is required. Because of the large storage capacity needed, a digital store is indicated. The above, as well as the following factors, weighs heavily in favour of a digital control system (i) Signals will have to be transmitted to the controiler over long distances, and digital transmission offers a high degree of noise immnity. (ii) A digital system does not suffer from any long-term stability problems 
(Chapter 2 - continued)

such as drifts in amplifiers, and so on.

(iii) A digital system offers the ability to achieve any desired degree of accuracy, once the signals have been digitized. Thus the storage of the setpoints and the error correction calculations can easily be carried out to an exact, specified degree of accuracy.

(iv) A digital control system favours the use of thumbwheel switches as setpoint inputs to the system. This means that these inputs can be specified exactly.

(v) Apart from the load cell amplifier outputs, all the other signals are digital (relay contacts, etc.)

\subsection{THE NEED FOR A DIGITAL COMPUTER}

When a designer is confronted with the task of developing a large digital controller which must have large data storage ability and be able to perform arithmetical operations, the suitability of a miniconputer must be thoroughly investigated. The advantages of incorporating a computer into a digital controller for this system were found to be:

(i) Flexibility. By software (programing) changes, the system can be adapted to incorporate any possible modifications. It can also be updated, if necessary.

(ii) Reliability. Extensive use of modern components, such as integrated circuits, together with many years of experience in their use, has given minicomputers a well-deserved reputation for reliability:

(iii) The fact that, despite vastly different applications, the basic hardware of a certain make of computer remains the same, means that the computer is much easier to maintain.

(iv) A computer can be used for more than one set task and will readily perform the function of co-ordinating such tasks.

(v) $/ \ldots$. . 
(Chapter 2 - continued)

(v) The computer can continuously keep track of variables over a long period of time. In many cases, it can detect process trends and slowly developing deterioration.

\subsection{WHY A MICRO-COMPUTER?}

Paragraph 2.2 justifies the use of a computer as the control element of this system. The next problem is the question of what type of computer is to be used. Investigation showed that the micro-processor would be a suitable choice, based on the following reasons:

(a) The functions of the weighing and feeder control system are relatively simple. It was unlikely that any software would be available for the chosen micro-processor so that any complex programming would count against the use of a micro-processor based system.

(b) Cost. It was estimated that the cost would be far less than that of any mini-computer based system available.

(c) The process is slow and has many dead-time periods. Most commerical micro-processors are considerably slower than the average mini-computer.

(d) Distributed control. The micro-processor is suitable for use in a distributed control system. This might consist of various micro-processor based controllers, controlling smaller processes within a plant. All these controllers could be linked to a central station, possibly mini-computer based, from where they would receive management information calculated on a global scale. Each micro-processor controlled "outstation" should be able to operate independently of the global input parameters. The central controller would also be used for all inventory functions and external control and/or access to the system. The advantages of such a system would be:

(i) The breakdown of any part of the system need not affect the successful operation of other portions.

(ii) The cabling to each local outstation would be minimized to possibly only one transmission channel. As the outstation is capable of independent operation, a break in this channel would not have catastrophic effects. 
(Chapter 2 - continued)

(iii) The cabling from the process to the nearest local outstation would be minimized.

(iv) anly software changes would need to be made to adapt the basic microprocessor based hardware to any local function it might be required to perform.

(e) Because of the use of large-scale integration, a micro-processor based system is likely to have fewer components, fewer connections and a much lower power dissipation. It will be more reliable and easier to maintain.

\subsection{THE MICRO-COMPUTER BASED SYSTEM}

It was decided to design and build a micro-processor based control system of which the basic "computer" hardware and software must be suitable to control a batch-weighing and raw material distribution system of the type used in arc fumaces.

A simulator was to be designed and built which would simulate the Witbank system. The processor was to be interfaced to it in order to demonstrate and assess the processor's capabilities.

The above system is known as the "prototype system", throughout this thesis. 


\section{CHAPTER 3}

\section{THE MICROPROCESSOR}

\subsection{HISTORY}

When the first two microprocessors were announced in 1971, an industry which had been predicted for at least a decade came into being. The major effect that this was to have on the electronics industry may be illustrated by some of the following quotes:

(i) "The microprocessors recently introduced by various semi-conductor companies foreshadow wide changes in the design of many electronic products and systems". $\therefore 7\}$

(ii) 'Microprocessors, to some degree, are causing computer history to repeat itself. The new areas in which microprocessors are being applied are as surprising and as imaginative as were the first minicomputer applications". '\{8\}

(iii) "The advent of the microprocessor is the beginning of a new computer revolution in our technological society". ' $\{9\}$

(iv) "The microprocessor has been designed as a sub-system into a startling variety of products and applications. The dollar volume growth from one year to the next appears to be about five-to-one in microprocessor and related component sales. Clearly, it has become a popular tool. Well over 100,000 will be shipped; this year (1974)... Commercially available microprocessors aren't yet five years old (1974) and there are already more than two dozen that the designer can choose from". ' $\{6\}$

The term "microprocessor" is usually given to a central processing unit (CPU) which consists of a control and arithmetic and logic unit (ALU) implemented in one or a few large-scale integration (LSI) packages.

$$
\mathrm{A} / \ldots
$$


(Chapter 3 - continued)

A microconputer is a microprocessor which has been interconnected to a memory and generally also to a control unit by which it can "communicate" with the outside world.

A microcomputer is distinguished from a miniconputer by:

(i) Much greater use of large-scale integration techniques

(ii) Lower cost

(iii) Lower power

(iv) Generally, but not necessarily, lower speed.

\subsection{ARCHITECTURE}

In considering the different microprocessor architectures there appear to be three major areas for investigation. ' $\{6\}$

(i) Software Design - covers the features of the microprocessor as seen by the progranmer

(ii) Hardware Design - includes the requirements of the environment in which the microprocessor must operate

(iii) Systems Design - affects the use of, and the interaction between, the software and hardware to achieve the required result.

(a) System Design

Some major features of microprocessors are:

Interrupt Structure

One-chip CPU Packaging

Direct Memory Access Ability, and

Arithmetic Modes

(i) Interrupts may or may not be important, depending upon two factors:

(1) does the application require real-time quick response to external events, and

(2) does the software design strategy encourage the use of interrupts? 
(Crapter 3 - continued)

Many applications don't require interrupts - even real-time applications. Instead of allowing main-line processing programs to be interrupted, software organization may assure the testing of some extemal status signal frequently enough to guarantee service.

(ii) One-chip CPU packaging has a substantial effect on assembly and repair costs as well as affecting the size of the finished product. Even among one-chip CPUs there are tremendous varieties on the number of ICs which must be added before achieving a working computer. A CPU that multiplexes $\wedge$ through the same pins as data requires several external packages to catch and hold a memory address. Most of the larger packages avoid this multiplexing. Other areas which frequently require substantial amounts of supporting logic are state decoding and $I / O$ control. The signals required to enable interrupts, enable and address input/output ports, write into storage and interrupt or reset the system, may be available at CPU pins or they may require as many as ten or twenty packages of decoding, storage and timing logic. While the parts costs, of these extra chips is relatively small, they can double or treble the manufacturing costs of the computer.

(iii) Microprogrammability allows the fine structure of the microprocessor to be changed, while the gross structure remains. In particular, the number of registers the programmer sees and the suite of instructions can be modified, within limited realms, but gross changes are not possible. Microprograms are usually stored in read-only memories, either on the computer's control chip or externally. Microprograming is important when a microcomputer is being designed that will emulate some other (more popular) computer, or when some specialised applications-oriented instructions need to be implemented to augment existing instructions set. Conmon but time-consuming software routines (such as multiply) can be implemented as microprogrammed instructions to speed up program execution. 
(Chapter 3 - continued)

Virtually any program can be reduced to a microprogram. Relatively complicated processing tasks (like Fast Fourier Transforms) will be executed much faster if implemented as a microprogram.

(vi) Direct Memory Access: this has been a popular option on ninicomputers for a long time. DMA allows high-speed peripheral devices to gain direct access to main storage without bothering the CPU: the alternative requires the software to read/write each and every word between main storage and the peripheral equipment. In order for DMA to work, the CPU must be prevented from interfering with main storage at the same time as the data transfer is taking place. Some microprocessors have special controls that suspend the CPU cycles (and, in fact, remove the device from the data/address bus by disabling three-state output drivers) whenever DMA is in progress. Processors that do not have an inherent DMA ability can be used in DMA applications, but the additional hardware is ofter complicated.

(v) Arithmetic: is almost always performed in two's-complement form on microprocessors. In addition, some processors have special instructions designed for handling $B C D$ numbers. Whether these instructions are important or not depends upon whether the input/output data must be in BCD form.

(vi) Vendor Commitment. Whether or not the vendor will continue making the microprocessor long enough to be of use during the life of the application is important. Furthermore, the manufacturer's commitment to the support of the product line can make life easy or difficult for the system-designer. Because of the complexity of these products, there are nearly always idiosyncracies that are uncovered only after several applications of the product have been completed.

(b) HARDWARE DESIGN

Microprocessors require other ancillary parts to make entire application systems work. As an absolute minimum, a microprccessor needs a program store 


\section{(Chapter 3 - continued)}

(although the Burroughs Mini-D has it on the CPU chip). Some microprocessors are easy to interface to storage, and others are hard. To the extent that the vendor has put all the necessary interfacing circuitry on the $\mathrm{CPU}$, memory and input/output parts, a family exists.

\section{Completeness:}

Completeness of the parts-family is important. Some vendors seem to feel that if the processor can be connected to a RAM, regardless of the number of additional chips involved, then their RAM is a member of the parts-family. Other semiconductor manufacturers have taken special pains to be sure that storage and processor input/output parts can all fit together without intervening logic; such systems are considered to have complete parts sets.

\section{(ii) Master Clocks:}

Clocks are required for microprocessors, although some of the newer processors only require a frequency-controlling two-terminal device (crystal or R-C net). Some of the vendors offer clock or clock-driver chips. In many designs, the DIP socket-sized crystal oscillators such as those from Motorola or Vectron are used. In others, crystal-controlled stability is not important. Generally, the clock frequencies do not have a direct relationship to the data manipulation speed of the processor. Note, however, that some processors require multi-phased clocks with critically inter-related timing relationships and awkward logic levels.

(iii) Power

Voltage and power dissipation are considerations in many systems. Many of the MOS processors require two supplies excluding ground (usually +5 and -9 or -10 volts). Power dissipation is most often a function of the intended operating speed range. 
(Chapter 3 - continued)

(iv) Compatibility

Most of the nicroprocessors offer some degree of TTL conpatibility, but numerous variations in the input/output logic levels are common - even on one chip. Some of the processors require an output buffer stage (sometimes low power. TTL) in order to be TTL compatible.

(v) Packaging

Package-size generally varies from 16 to 48 dual-in-line (d.i.1.) pinned packages. In general, packages with fewer pins would be easier to interconnect, while packages with a large number of pins would be easier to interface to.

(c) SOFTWARE DESIGN CONSIDERATIONS

After the system is designed, and the hardware has taken shape, someone has to program the microprocessor. What the programmer sees is 1argely determined by the architecture of the computer and the environment in which that native architecture operates.

Word Size

Word size affects the microscopic efficiency of some common kinds of software operations but it seldom affects the overall throughput of the design. In many applications, four-bit processors out-perform similar designs on eight-bit processors, although the opposite is also true.

In general, ease of programming is inversely proportional to:

(1) Word size, because smaller data word sizes require multi-word operations on practical data quantities, and

(2) The number of different register sizes in a computer, because simple register-to-register data transfers are limited.

Computers with small word-sizes (four-bit) and to a lesser extent eight-bit) always require a different register size for storing some addresses. For example, the program location counter is often made an integral number of native words in size. When the word size is small, the manipulation of storage addresses $/ \ldots$. 
(Chapter 3 - continued)

addresses and program addresses is complicated. Hence, ease of programming and not necessarily the overall application efficiency is what is influenced by word size.

(ii) Addressing Capacity

How large a program can be written without resorting to special external hardware and internal software techniques is defined by the programaddressing range. The ideal processor for an application has neither too sma11 nor too large a capacity. A too small range means that extra hardware will be required to extend the addressing. On the other hand, excessive capacity means that extraneous address bits will be carried in every instruction that refers to storage.

(iii) Addition Time addition/

Register-to-register ${ }_{\Lambda}$ time is a popular estimate for the computing speed of a computer. This instruction is chosen as a selection factor because nearly every computer has an add instruction. Microprocessors with more than one progranmer-accessible register for data manipulation on the CPU chip can perform a fast regișter-addition in a minimum instruction execution time. Some processors, however, are organised as one-accumulator computers so that register-to-register additions are not provided; in this case, the addition of the contents of an arbitrary storage location to the accumulator is often scored as the minimum addition time.

(iv) Register Complement

The number of registers in the microprocessor is probably the most important feature of its architecture. Different registers in a computer have different uses; the ways that they may be used are embodied in the instruction set. The most precious resource that the programmer can allocate in software is the set of CPU registers. The more registers there are, the less likelihood there is of main storage references. Generally, references to main storage are more expensive in time than the references to on-chip registers. 
(Chapter 3 - continued)

Arithmetic (or ALU) registers are those on which ALU functions can be performed, the register can be a source or destiration of operands for the operation. Registers that can supply but not receive operands for the ALU are not considered arithmetic registers. An index register is a programmeraccessible register that is implicitly included in certain references to main storage. Unless the contents of the register can be added to another value (from, say, the instruction $i t_{s e 1 f}$ ) during the storage addressing cycle, it is not an index register.

All other programmer-accessible data registers to a microprocessor (excluding the ALU and index registers) are called general purpose or scratch-pad registers. (In some large-scale computers a general-purpose register can be used in any of these three modes; this practice is not popular' in microprocessors).

(v) Return Stack

Return addresses are usually handied through the medium of a push-down stack in microprocessors. Except for the Intersil 6100, which emulates the DEC PDP-8, microprocessors put subroutine return addresses into a push-down stack in a read-write memory; this memory may be on the CPU chip or external main storage. Return addresses are not saved within the program storage areas as in many minicomputers because most programs for microprocessors are stored in read-only memory.

When the return addresses are stored in an on-chip push-down stack, there is some natural limit to the number of dynamic subroutine calls. If there are eight stack positions, then generally only seven subroutine calls may be active at one time; if interrupts are anticipated, the real stack size must be kept smaller to allow some stack depth for the interrupt service routine. When return addresses are stored in RAM, an on-chip stack pointer is maintained in the CPU, when a subroutine is called, the return address is pushed into the RAM stack, and the pointer is updated. 
(Chapter 3 - continued)

The on-chip stack allows faster subroutine cal is because RAM does not have to be accessed. Stacks in RAM are of potentially huge depth, and this allows certain kinds of algorithms to be easily programmed. If the on-chip stack is accessible to the programer, the depth of that stack can be extended by software. Most on-chip stacks are not accessible, and this imposes a rigid limit on the allowed depth of subroutine calling.

(vi) Addressing Modes

The addressing of data and program segments is an area that presents enormous variations from computer to computer. The problem is to allow references to be made to any arbitrary word in the addressing space, yet eliminate the need for a full set of address bits in each and every instruction. If the full address is required, program sizes tend to grow. Most microprocessors take advantage of the fact that most data program references are local in scope; that is, references are mot often close to the address of the current instruction being executed or close to the last datum reference in main storage.

Any of the following addressing modes may be found in different microprocessors: direct, abbreviated (only part of the address is carried

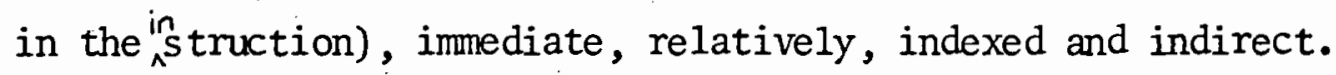

\subsection{APPLICATIONS}

The applications area of the microprocessors may be broken up into three major categories ' $\{10\}$

(i) To replace hard-wired random logic

(ii) To replace minicomputers in certain applications

(iii) Totally new applications

(a) Replace Some Minicomputers

Microprocessors were originally not intended to replace minicomputers. They were meant to provide an alternative to hardwired logic design. However, microprocessors/..... 
(Chapter 3 - continued)

microprocessors now form a viable alternative to minicomputers when only limited computation and control are required.

(b) Replace Hard-wired Random Logic

The experience gained by Tektronics $\{10\}$ shows that a microprocessor may profitably be used when the number of medium-scale integration (MSI) and single-scale integration (SSI) packages necessary to implement the controller with random logic amount to more than $30-40$. In the United States' $\{11\}$, a microprocessor and one kiloword of memory $y_{\Lambda}$ replace about 50-100 IC's, and this reflects a cost saving of about $75 \%$. Nicroprocessors may replace hard-wired random logic in existing products as well as in products still in the design phase. In some cases, the advantages of microprocessors compensate for the trouble of designing the extra software cycle. (c) New Applications

The microprocessor has brought with it a whole new era of products. We now hear of "intelligent" instruments, "intelligent" interfaces, and "intelligent terminals. The concept of adding "intelligence" to traditionally "dumb" products is a very exciting one, since it provides possibilities remote in the past.

Examples of the fields in which microprocessors are used today:

(1) Point of sale terminals

(2) Grahpic terminals

(3) Remote data entry terminals

(4) Industrial systems:

Capacitance bridges DVM's Synthesizers Waveform-processing instruments

(5) Process and numerical control systems

(6) Aircraft control systems

(7) Traffic controllers

(8) Peripheral controllers 
(Chapter 3-continued)

(9) Inventory systems

(10) Credit card verification systems

(11) Medical instrumentation 


\section{CIAPTER 4}

\section{PROPOSED SYSTEM}

Extensive investigation showed that the following system would have the characteristics desired of a microprocessor-based, batch-weighing and raw-material distribution system of the type used in arc furnaces.

The processor should be able to control a system having the following maximum specifications:

(1) No. of weighing hoppers (with feeders) : 3

(2) No. of holding bins (with feeders) : 12

(3) No. of electrodes : 3

(4) No. of furnace hoppers per electrode : 5

(5) The destination of the raw material feed-mix should be any selected furnace hopper.

(6) The routing control should be capable of being adapted to any system similar to that of the Witbank system.

(7) Moisture compensation for one material.

(8) Load cell input to processor is 13-bit BCD with a resolution of 1 in 1999.

(9) Each furnace hopper can be considered as a different zone and can have a special raw-material recipe.

(10) Taring error compensation.

(11) Overshoot error compensation.

(12) The whole system should not be shut down after each weighing cycle when unnecessary.

(13) A terminal device (such as a teletype) should be used for. inventory control Input/Output with the system.

(14) An operator control panel should be used for simplified control of the system.

(15) The existing conventional type of system must be modified as little as possible and, in particular, it should be possible to switch over to the existing emergency, normal-feed and weighing standby.

A more detailed description of some of the desired characteristics follows:

4.1 THE WEIGHING HOPPERS AND THEIR FEEDERS

The analogue outputs from the weighing load cells will have to be digitized to supply digital readout panel meters as well as the 13-bit $\mathrm{BCD}$ (binary coded decima1) imputs to the processor. The feeders which empty the weighing hoppers are thyristor-controlled vibration feeders. They will be turned on and off from the processor, but as the ratios of the materials, s particularly weighing hoppers, vary very little for a specific 
(Chapter. 4 - continued)

set-up, their feeding rates will remain manually adjustable.

4.2 THE HOLDING BINS AND THEIR FEEDERS

The system as such will not check the levels of the raw materials in the holding bins, but this can be incorporated via an alarm port which will be discussed later. The feeders feeding out of the holding bins into the weighing hoppers will, except for their feeding rates, be completely controlled from the processor in their fine and coarse feeding modes.

\subsection{THE FURNACE HOPPERS}

In conventional systems there are four furnace hoppers around each electrode. It was decided to incorporate facilities in the processor to allow for the addition of an extra furnace hopper at each electrode. This could then be used to make quick local corrections within the furnace. These furnace hoppers would be especially designed so that they could always be empty (or nearly empty), thus minimizing the time before the corrective batch reached the active region in the furnace.

\subsection{THE ROUTING}

The processor will output a signal, selecting one of the furnace hoppers. The conventional control system (such as exists at Witbank) will then be used to control the belts and shuttles so that the route is set up to the selected furnace hopper. The processor can assess this route via the data input bus to check if the route is correct and working (i.e. all the belts up to speed) before releasing the feed-mix from the weighing hoppers on a specific occasion or at any time thereafter.

\section{.4 .5 MOISTURE COMPENSATION}

One of the raw materials used, coke, has an average moisture content of $5 \%$, but this can rise as high as $16^{\circ}$. It is therefore necessary, when weighing the coke, to compensate for this discrepancy, in order to ensure an alloy of the desired quality. Typically every $100 \mathrm{~kg}$ of feed-mix contains about $22 \mathrm{~kg}$ of coke.. $\because 16 \%$ moisture in the coke equals 
(Chapter 4 - continued)

$$
\frac{16}{100} \times 22=3,52 \mathrm{~kg} \text { in } 100 \mathrm{~kg} \text { ore }
$$

$\therefore$ the relationship between weighing accuracy and percentage moisture is (for a weighing accuracy of $0,5 \%$ ):

$$
\frac{0,5 \times 22}{100} \times \frac{100}{3,52}=3,18
$$

With this accuracy required of the moisture analysis, an input of 8 bit $\mathrm{BCD}$ number for the : moisture content to the processor was chosen. This gives a resolution of 1 in 99 or $1 \%$.

\subsection{TARING ERROR}

Before a weighing hopper is filled, the processor checks the "zero weight" and ....... makes ... ... a taring error correction, :

\subsection{OVERSHOOT ERROR}

Overshoot errors exist because after the feeders feeding the weighing hoppers have been switched off, more material might fall into the weighing hopper. After switching off a particular feeder, the processor therefore waits for a suitable settling-down time and checks the weight in the weighing hopper. It uses this weight to make corrections in a similar way as for taring error correction.

\subsection{SHUT-DOWN SYSTEM AFTER EACH CYCLE?}

In $a$ conventional systems of this type, the whole system (including conveyors, and so on) shuts down after each cycle, even if the same furnace hopper is due to be serviced again. Such a process is very time-consuming, since the controller must wait for all the conveyors to come up to speed. The processor does not shut down the system but merely changes the required conditions, therefore no time is wasted if the same furnace hopper is to be serviced again. 
(Chapter 4 - continued)

\subsection{EXTERNAL COMMNICATION}

The external control elements to and from the system are to be as follows:

(i) An operator-controlled panel to start, stop, set initial setpoints, and so on.

(ii) A teletypewriter to print and/or punch onto paper tape the inventory and management information, as required. The teletypewriter will also be used as a more sophisticated input device of some control parameters.

Setpoint input to the processor: the operator will specify the initial and "average" required setpoints (recipe applicable to all the furnace hoppers) on thumbwheel switches. These are input into the processor on request from the control panel. Any special recipes for a particular furnace hopper or group of fumace hoppers and the number of changes for which they are required can be entered from the teletypewriter. This entry takes priority over the thumbwheel settings.

Inventory information will be as follows:

(i) An automatic 8-hourly shift report will be made which will consist of the following:

Original setpoints and any alterations;

Time of any report or alteration;

Sum of the weight of materials used for each holding bin;

Sum of the weight of materials (total mixture) fed into each furnace hopper.

(ii) Any of the above can be requested from the teletypewriter at any time.

(iii) Provision for any of the above to be punched onto paper tape for processing elsewhere.

4.10 INTERFERENCE WITH EXISTING CONVENTIONAL SYSTEMS

Interference with, and interconnection to, typical conventional systems will then be as follows. The outputs of the load cell amplifiers have to be conditioned, digitized and multipiexed onto the processor!s 
(Chapter 4 - continued)

input bus. The signals from the processor, selecting which furnace hopper is to be serviced, are to be converted and connected to the existing routing control. The signals from the process, i.e. "shuttle position", "belt up to speed", "furnace hopper empty", and so on, have to be conditioned and multiplexed on to the processor's input bus. 
SECTION 2.

SYSTEM HARDWARE. 


\section{CHAPTER 5}

AN INTRODUCTION TO THE HARDWARE

\subsection{GENERAL}

The prototype microprocessor-based systenl was designed to meet the following requirements:

(i) Be capable of establishing the feasibility of the project.

(ii) Be capable of evaluating the ultimate requirements of such a system. In other words, it should establish the specification for a final production model.

(iii) It should not necessarily be considered as a pre-production model and therefore need not be in an industrial form.

The prototype which was developed consists of the following sections (see Figure 5.1):

(i) A processor. This conists mainly of:

(1) An Inte1 $8008 \mathrm{microprocessor}$ (the 8008)

(2) Eight kilowords of eight-bit memory

(3) A front panel

(4) An interface to a minicomputer

(5) Process interfacing

(6) A teletype interface

(ii) Software development aids. This consists of:

(1). A direct menory access channel through which the processor's memory can be lcaded from a minicomputer - a Varian 620/L-100.

(2) A peripheral device from which any memory location can be changed or displayed. This device also uses direct memory access techniques.

(iii) A simulator (see Chapter 9). This is a digital system designed to simulate, as closely as possible, the operation of the Witbank system. This was designed to enable the programmer to test processcontrol software and to evaluate whether the chosen microprocessor is fast enough to cope with the demands of the system. 


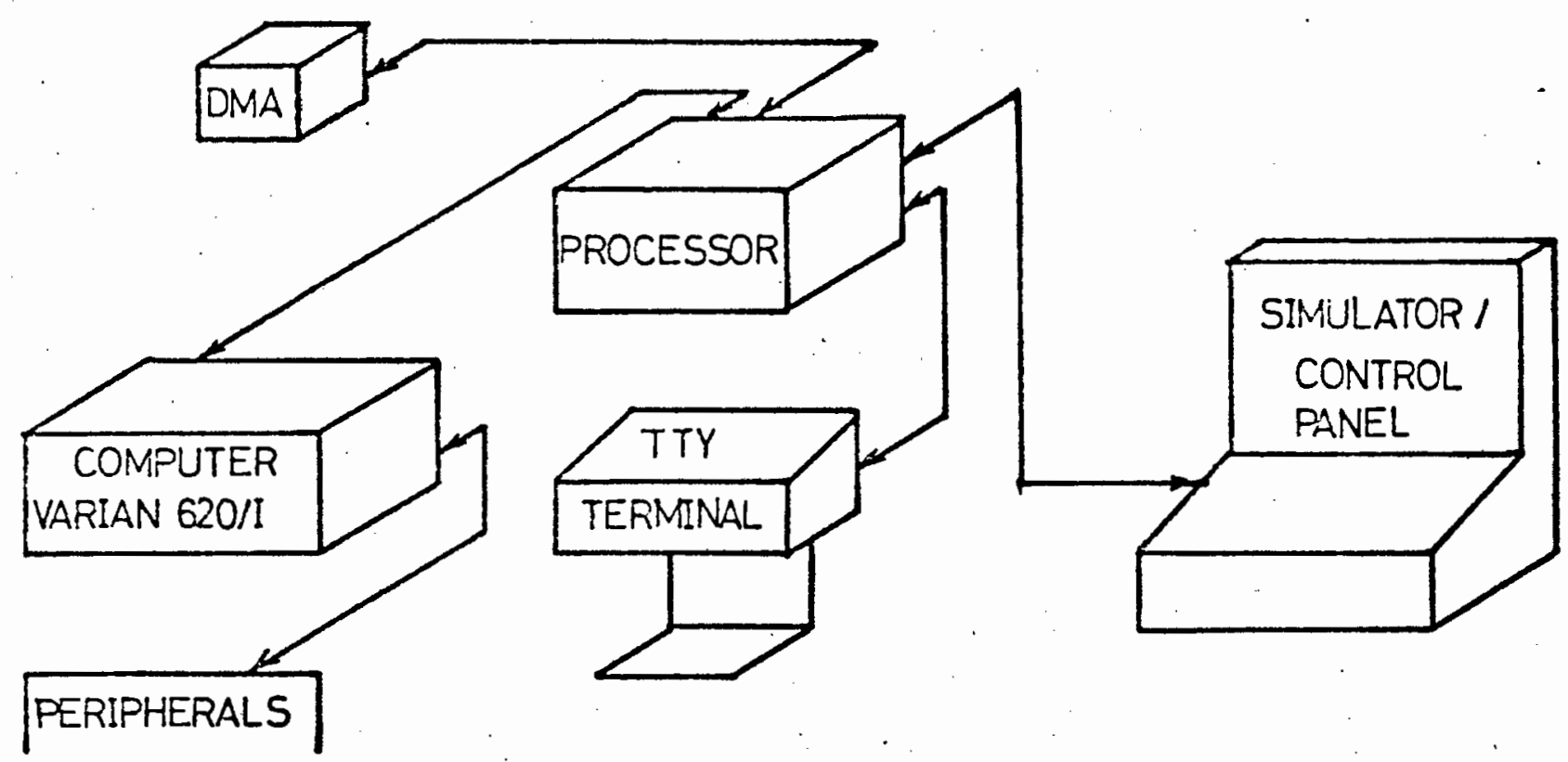

FIGURE 5.1 PROTOTYPE WITH DEVELOPMENT AIDS : SYSTEM INTERCONNECTION.
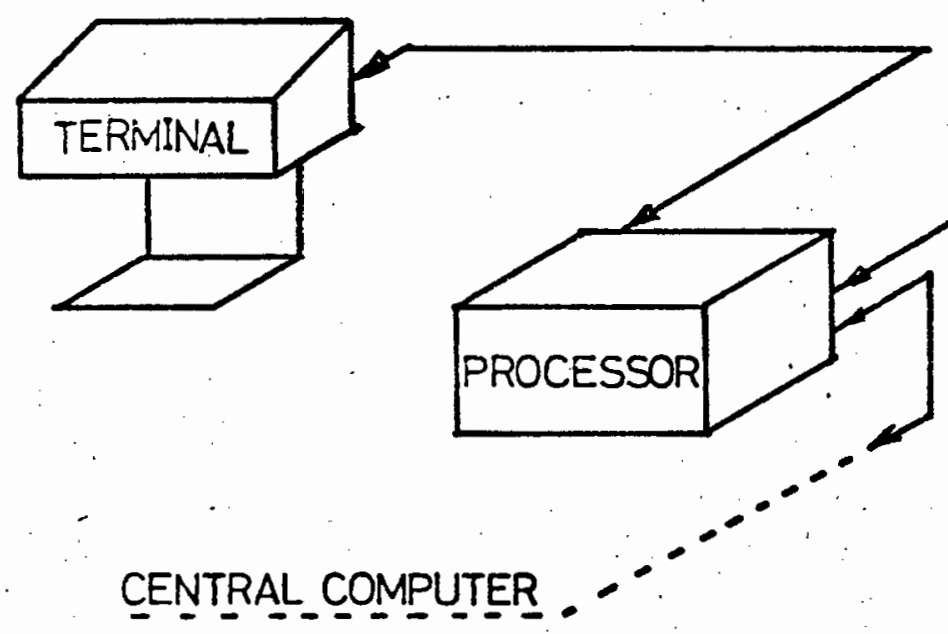

RAW MATERIAL WEIGHING AND DISTR, SYSTEM

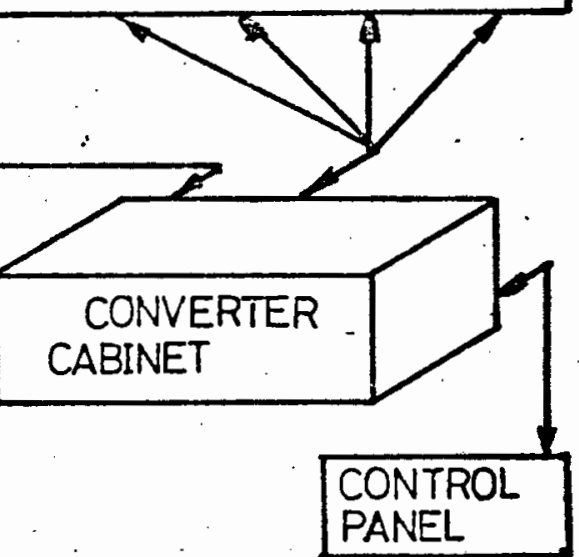

FIGURE 5.2 PROPOSED LAYOUT OF THE SYSTEM WHEN INSTALLED ON THE PLANT. 


\section{(Chapter 5 - continued)}

(iv) An operator control panel. For the prototype, this was combined in one cabinet with the simulator.

\subsection{INTEGRATING THE PROTOTYPE WITH THE REAL PROCESS}

In order to use the prototype as the control element in the actual system, the following aspects were considered. (See Figure 5.2).

(i) Replacement of the simulator by the real process. The system was designed so that the cable connecting the processor with the simulator/operator's control panel would be used to connect to a converter cabinet. From this point, the system would be inter-connected to the operator's control panel and to the process. The inter-connecting cable can be kept short so that no special transmission techniques would be required. All the necessary signal conversions to obtain the signal and noise immuity would be done in the converter cabinet.

(ii) Replacement of the software development aids. The memory is structured in such a way that the control program can be replaced by read-only (ROM) memory which is non-destructible. The control variables and inventory information will remain stored in random-access (RAM) mamory. In this way, the DMA channel to a minicomputer and to the peripheral device would no longer be required. Note that the processor is designed to incorporate the facility of obtaining the management information directly from a minicomputer.

\subsection{SELECTION OF ELECTRONIC COMPONENTS}

The choice of logic family for the prototype was transister-transisterlogic, TTL. This decision was based on the following points:

Relatively inexpensive

Fast enough to be compatible with the rest of the system Readily available

Available with a range of logic functions

Most current microprocessors are designed to be TTL compatible. Suitable memory components exist which are TTL compatible. 
TTL, and more specifically the $54 / 74$ series, has many compatible logic fanilies to which the prototype could easily be changed if this were found to be necessary.

The choice of microprocessor to be used was made during the first quarter of 1974. The choice of microprocessors at that time (especially on the local market) was reasonably limited. (See Appendix 1)

The Intel 8008 was chosen because:

(i) Lack of ROM programming facilities and of a thorough knowledge of microprograming techniques weighed heavily against the use of micro-progranmable microprocessors.

(ii) The application favoured the use of interrupts which are readily handled with an 8008 .

(iii) It was estimated that the 8008 was fast enough for the current system. The Intel 8080 , which is ten times faster than the 8008 , was not readily available. It was therefore decided that if the 8008 proved too slow the system could always be upgraded to an 8080 . The 8080 is compatible with the software required for the 8008 .

(iv) The 8008 input lines are TTL-compatible and its output lines only require low-power TTL - buffers for TTL compatibility.

(v) The 8008 is widely used in industry in the United States of America and has therefore proved its reliability. In fact, it is one of the few standard microprocessors in industry.

\subsection{THE INTEL 8008 MICROPROCESSOR $\{6\}$}

The following brief sumnary of the Intel 8008 microprocessor delineates some of its characteristics. (Appendix 2 gives the manufacturers' full description and specifications).

The 8008 is an eight-bit microprocessor available in an eighteen-pin dual-in-line package.

It can add two eight-bit numbers in 20 microseconds. 
(Chapter 5 - continued)

Internally, the Centrol Processor is arranged around seven registers, one of which is the accumulator. The accumulator is used in the arithmetic operations and with input/output instructions. Two of the registers are used for all references in main storage. In order to address a specific word in memory, the program must load these two registers individually. This implies that at least three instructions must be used to refer to a single location in memory.

The 8008 has a relatively simple interrupt mechanism. When the interrupt signal is used, the processor inhibits incrementing the program counter, issues an acknowledgement, and proceeds through the normal instruction fetch. The external interrupt hardware "jams" in a substituted instruction, usually a sub-routine call that simultaneously saves the program counter and invokes the interrupt service routine,

In most computers, when an external event causes an interrupt, the can be/ contents of the processor registers saved in main storage. These registers can then be used by the intermpt service sub-routine. In the 8008 , the register pair used for storage addressing may already contain values established before the interrupt occurred, so that they cannot be disturbed. The system designer can choose to reserve two of the general purpose registers as interrupt registers (into which the storage addressing registers can be placed during interrupt servicing). This implies the reservation of two of the seven registers available. In the case of the prototype, it was decided to use two external hardware registers (loaded and read via input/output instructions) for this purpose. (See 6.7).

Thus it can be seen that a major disadvantage of the 8008 is the addressing scheme which requires programing addresses directed into a specific register pair.

\subsection{A FUNCTIONAL DESCRIPTION OF THE PROCESSOR}

Figure 5.3 is a block schematic showing the relationships between the different sections of the prototype. All circuitry inside the dotted line is built inside the processor cabinet. The blocks in the schematic are 


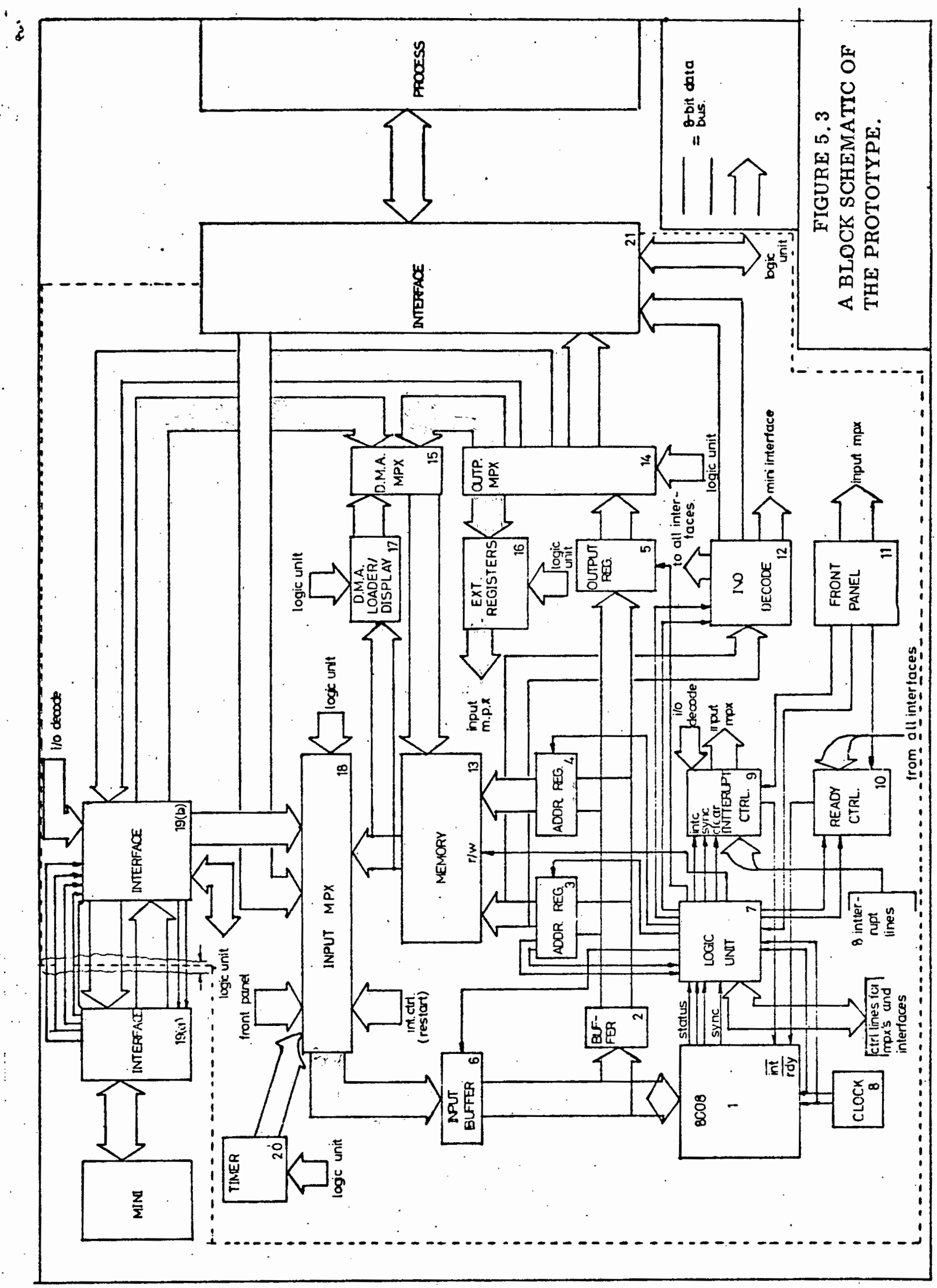


(Chapter 5 - continued)

referred to numerically.

The 8008 (1) is the heart of the processor. It has an eight-bit input/output data bus (shown shaded). The 8008 is driven from a two-phase clock (8) and its two control inputs are an interrupt line (int) and a ready line (rdy). The ready line can be used to cause the 8008 to "wait" for any desired period between the address and data phases of its normal operational cycle. The 8008 has three status output lines which display the internal processor state, and a synchronisation line (sync).

On output, the data bus is buffered by, and fed to, the address registers (3) and (4) and to the data output register (5). The six higher order bits (HOB's) of address register (3) can be decoded by the input/output controller (12) to select the appropriate external device. The two highest order bits are decoded by the logic unit (7) to indicate the current machine cycle, i.e. memory read, I/0, memory write.

On input, the correct byte of data is selected and fed to the input buffer (6) via input multiplex (18) circuits which are controlled from the logic unit (7). The data on the input buffer is selected at the correct time by the logic unit and placed on the 8008 data bus.

The "ready control" (10) causes the 8008 to enter a "wait-state" whenever any external device has not completed its required function in time to meet the requirements of the 8008 's timing cycle. The uses of the front pane1 (11) are:

(i) To stop program execution

(ii) To start program execution at a specific location

(iii) To "step" through a program one cycle at a time

(iv) To display the current processor condition

The timer (20) enables the programmer to estimate short periods of time without having to use "marking time" sub-routines.

The interface (19) to an extemal minicomputer is divided into two sections (19(a) and 19 (b)). 19 (b) is built into the processor and is 
(Chapter 5 - continued)

designed so that it can interconnect to any type of minicomputer using an appropriate interface (19 (a)) 


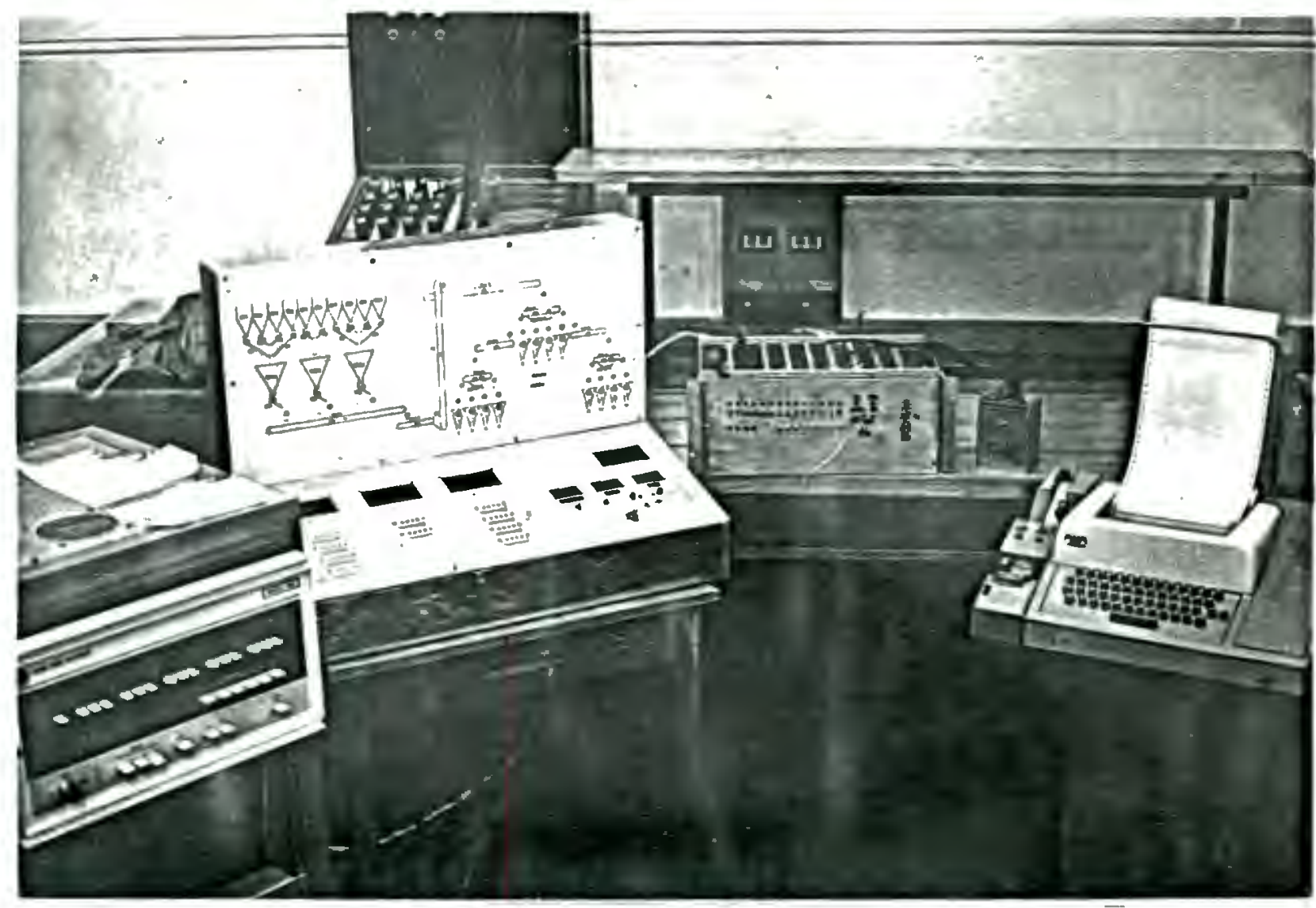

(a)

(b)

(c)

(d)

(e)
(a) VARIAN 620/L-100
(b) SIMULATOR \& CONTROL PANEL
(c) PROCESSOR
(d) DMA LOADER/ DISPLAY
(e) TELETYPE 


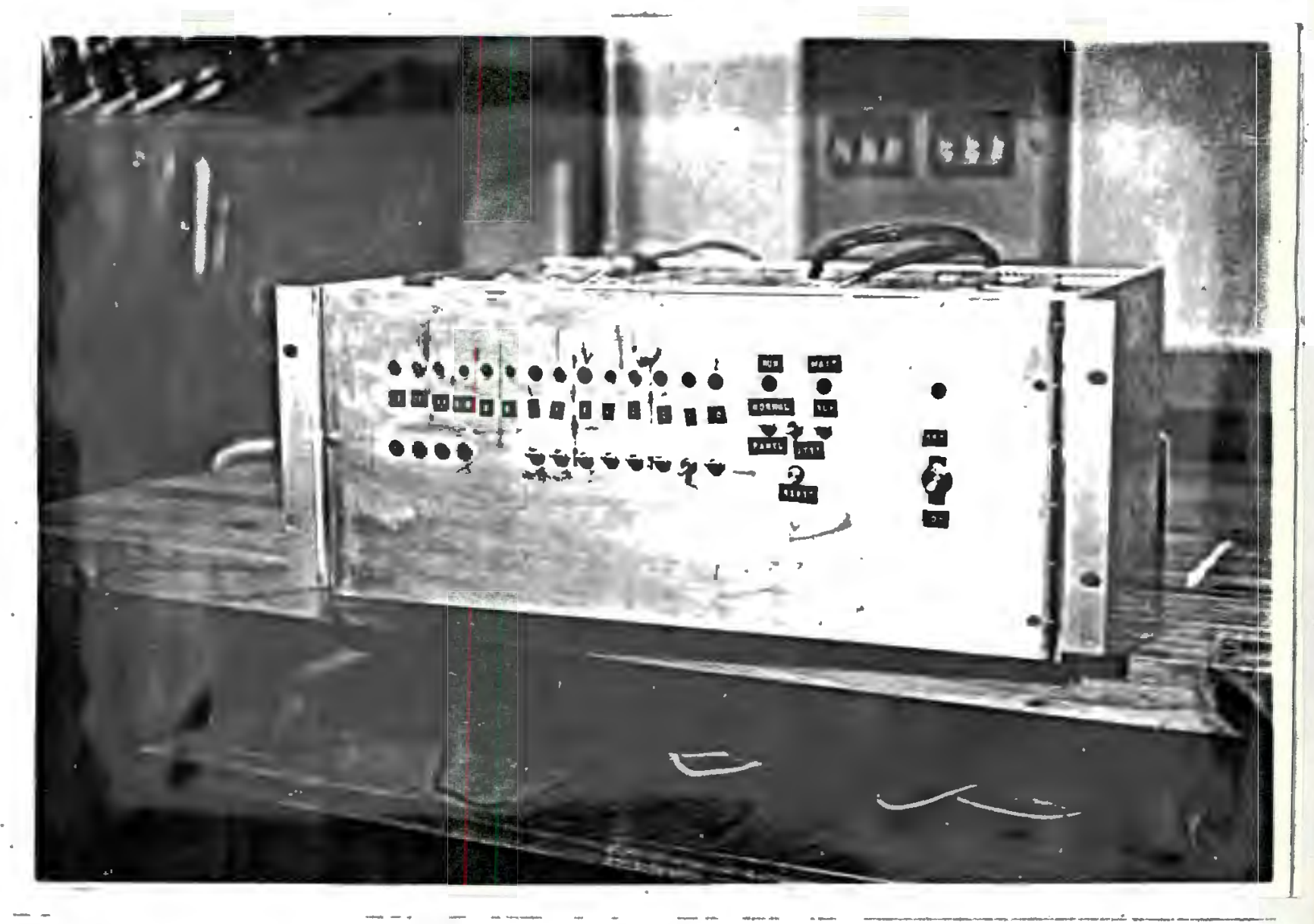

THE PROCESSOR

PLATE 2 


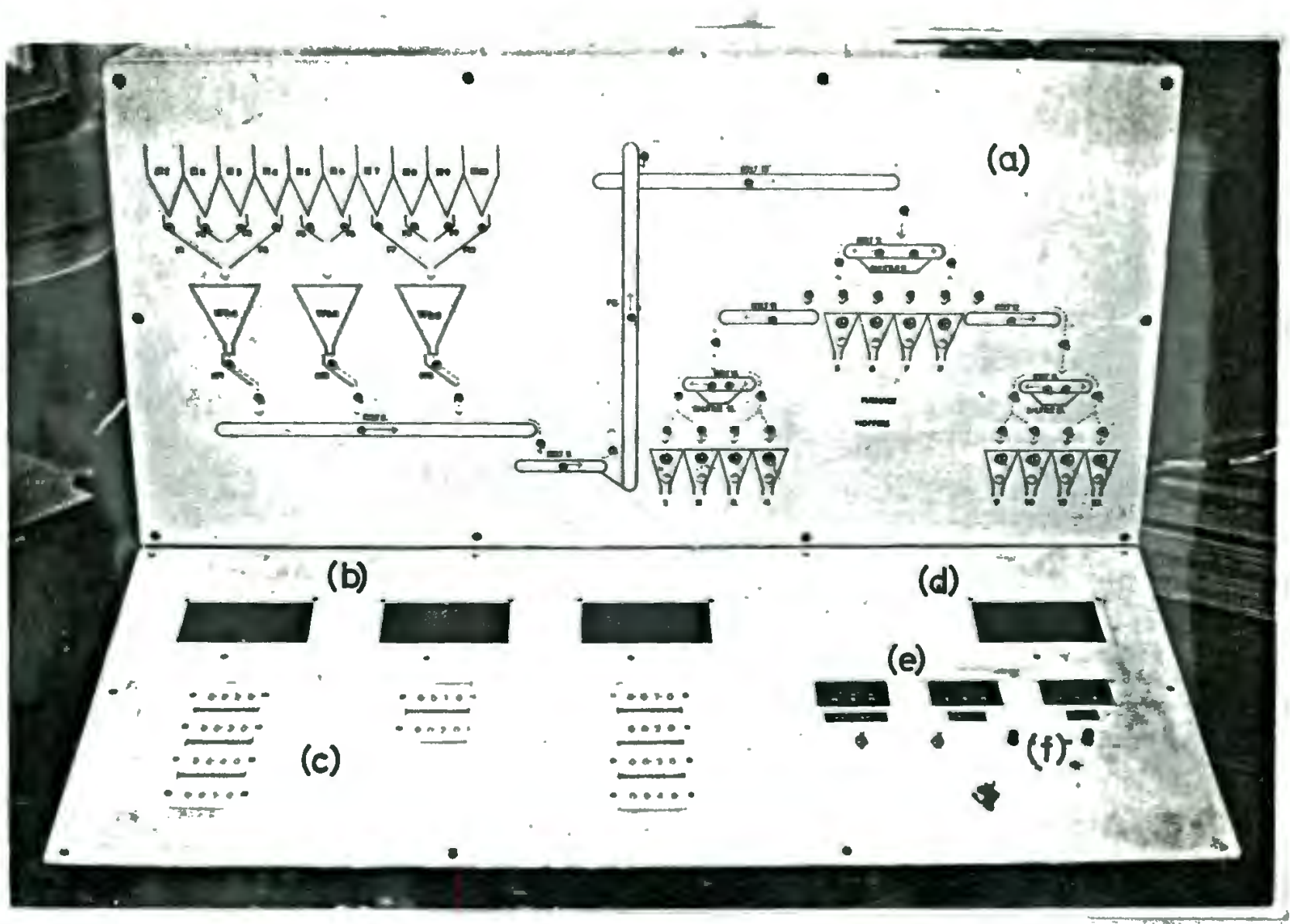

(a) MIMIC PANEL

(b)LOAD CELL OUTPUTS

(c) SETPOINTS

(d) TIME-OF-DAY CLOCK

(e) MOISTURE \& TARE

(f) START \& RESTART

PLATE 4 

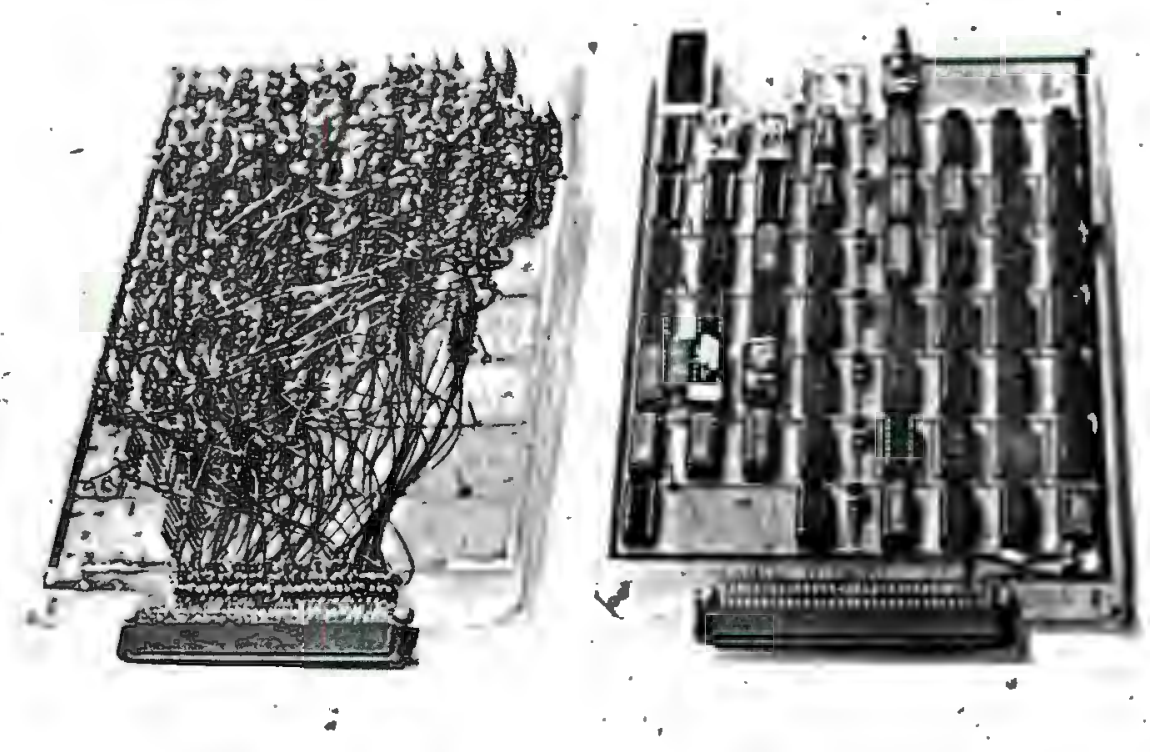

A TYPICAL CIRCUIT CARD

PLATE 3 


\section{CHAPTER 6}

THE PROCESSOR

\subsubsection{THE 8008 AND ITS CONTROL CIRCUITS}

The 8008 and its control circuits are constructed on one card occupying slot number one in the main processor rack. The circuit of this card is shown on Figure 6.1. Familiarity with the 8008 (Appendix 2) will facilitate understanding of the descriptions which follow.

External control over the 8008 is via the RUY and $\overline{\text { INT }}$ lines. In turn, the 8008 exercises control the entire system, using its state lines (SO, S1, S2) and two control bits (CBO, CB1) which are output on its data bus during every T2-state.

The state lines are continuously decoded by DI into one of the processors states (TI!, T1, T2, W, T3, S, T4 or T5).

The control bits are stored in register Feglusing the signal $\mathrm{T} 2 \cdot \emptyset_{22}$ (gate Al). They are then decoded by D2 to reveal the current machine cycle being executed.

The four possible machine cycles are:

1) PCI Designates the address is for a memory read (first byte of instruction)

2) PCR Designates the address is for a memory read (additional bytes of instruction)

3) PCC Designates the data as an input/output operation command.

4) PCW Designates the address is for a memory write data.

The SYNC output from the 8008 and the signals from the two-phase dock $\left(\emptyset_{1}, \emptyset_{2}\right)$ are decoded by D3 as follows:

$\emptyset_{11}=\operatorname{SYNC} \cdot \emptyset_{1} ; \emptyset_{12}=\operatorname{SYNC} \cdot \varphi_{1} ; \emptyset_{21}=\operatorname{SYNC} \cdot \emptyset_{2}$ and $\emptyset_{22}=\operatorname{SYNC} \cdot \emptyset_{2}$

These signals and the SYNC signal are used to obtain the correct timing relationships in the microcomputer system.

During the $\mathrm{T} 1$ (or T1I) state, the data appearing on the 8008 's data bus is stored in the LOB address register REG 2. The signal $(\mathrm{T} 1+\mathrm{T} 1 \mathrm{I}) \cdot \emptyset_{22}$ is used as the clock signal (gates 01 and A4). 


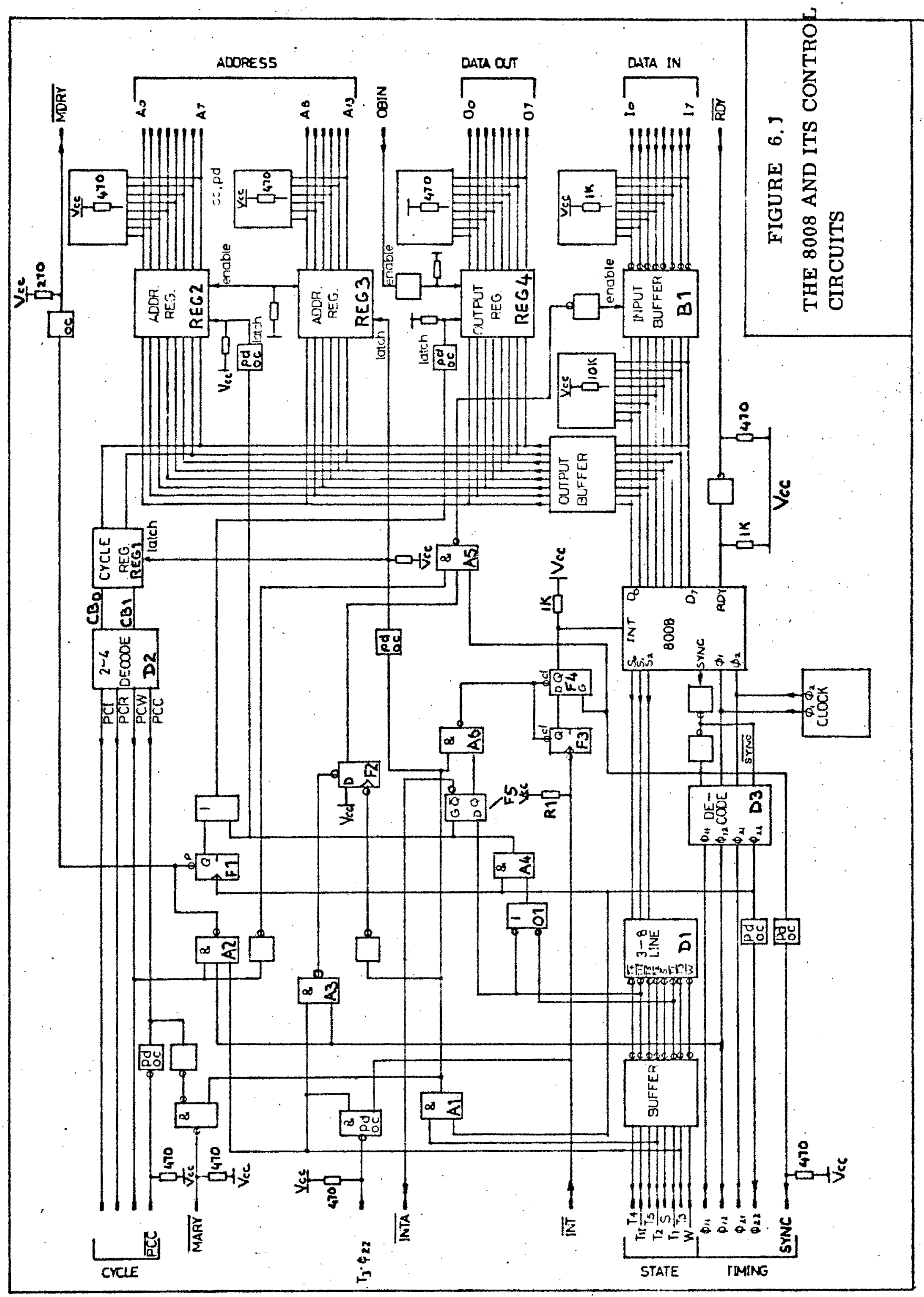


(Chapter 6 - continued)

During the T2 state, the data is stored in the HOB address register, REG 3, using the signal T2 $\cdot \varphi_{22}$. Note that the two highest order bits are stored in a separate register REG 1 to supply the control bits as previously described.

Data output from the 8008 is via REG 4. The data can be loaded into this register during two of the machine-states (T1 (or TII) and T3). When the 8008 requires to output data to its memory (PCW-Cycle), the data is made available during a T3-state. Register REG 4 is latched to receive this data during the period when the signal T3 $+\mathrm{PCW} \cdot \emptyset_{12}$ (gate A2) first appears until the end of the next $\emptyset_{22}$ signal (flip-flop FI). When the 8008 outputs data via an instruction (PCC-cycle) the data is made available during the T1 (or T1I) state. Register REG 4 is latched to receive this information using the signal $(\mathrm{TI}+\mathrm{T} 1 \mathrm{I}) \cdot \emptyset_{22}$.

Data input to the 8008 is via buffer B1. The state-output lines can have a delay of up to 1.1 microseconds before indicating the T3-state. The data input to the 8008 must appear on its data lines before or with the falling edge of $\emptyset_{11}$, during the T3-state. This means that the system is required to anticipate an imminent T3-state when data input is required. Therefore, the rising edge of the signal $\emptyset_{22} \cdot T 2$ is detected (flip-flop F2) and then "and-ed" with SYNC and PCW to achieve this (gate A5). The signal T3 $\emptyset_{12}$ (gate A3) is used to clear flip-flop F2.

Flip-flops $F_{3}$ and F4 are used to synchronize an incoming interrupt with the 8008 's clock signals. The 8008 recognizes the interrupt during its next instruction fetch cycle by the output of a TII-state in place of a T1-state. This is recognized by flip-flop F5. The outputs of F5 are used to indicate to the peripherals that an interrupt is being acknowledged (INTA) and to clear the flip-flop $F_{3}$ and $F 4$ via gate $A 6$. The pull-up resist, $t_{\lambda}$, is included so that intermpt requests ( $\overline{\text { INT }}$ ) may be "dot-ored" together, using opencollector techniques. 
(Chapter 6 - continued)

The signal, $\overline{\mathrm{T3} \cdot \emptyset_{22}}$, indicates to the peripheral controllers that one cycle in which they may be required is finished. $T \overline{3} \cdot \varphi_{22}$ is thus used as a reset signal for these controllers.

The signal, $\overline{M A R Y}$, indicates to the memory circuits that a valid memory address has been placed on the address bus, $A_{0}-A_{13}$.

The signal, $\overline{\mathrm{MDRY}}$, indicates to the memory circuits that valid data which must be stored exists on the data output bus, $0_{0}-0_{7}$.

Note that in the design of these circuits an effort was made to facilitate a changeover to an 8080 microprocessor if this was found to be necessary for reasons previously discussed. (See Chapter 5.3).

\subsection{THE FRONT PANEL AND ITS ASSOCIATED CIRCUITRY}

The light-emitting diodes (1.e.d.s.), their drivers, and the switches are located on the front panel; the associated circuitry is built on card two (slot two). Figure 6.2 shows these circuits.

The switches in the top left-hand comer are shown for the processor in the "RUN" mode, i.e. its normal functional mode.

With the toggle switch, S1, in the "RUN" position, ready pulses, $\overline{(\mathrm{RDY})}$, are supplied to the 8008 (via gate Al) using the signal, $\overline{\mathrm{PCC}} \bullet \emptyset_{22}$.

From this it can be seen that except during input/output operations ready pulses are automatically supplied; hence

(i) The memory used is fast enough to ensure that the 8008 will never be retarded by memory transfers.

(ii) As the ready signal (RDY) is inhibited during input/output (PCC-cycle) operations, all peripheral controllers have to supply the 8008 with a suitable ready signal when data transfers are taking place.

With S1 in the "STEF" position, the ready pulses are supplied via a spring-loaded switch, S2, and monostable M1, thus providing a single-step facility. 

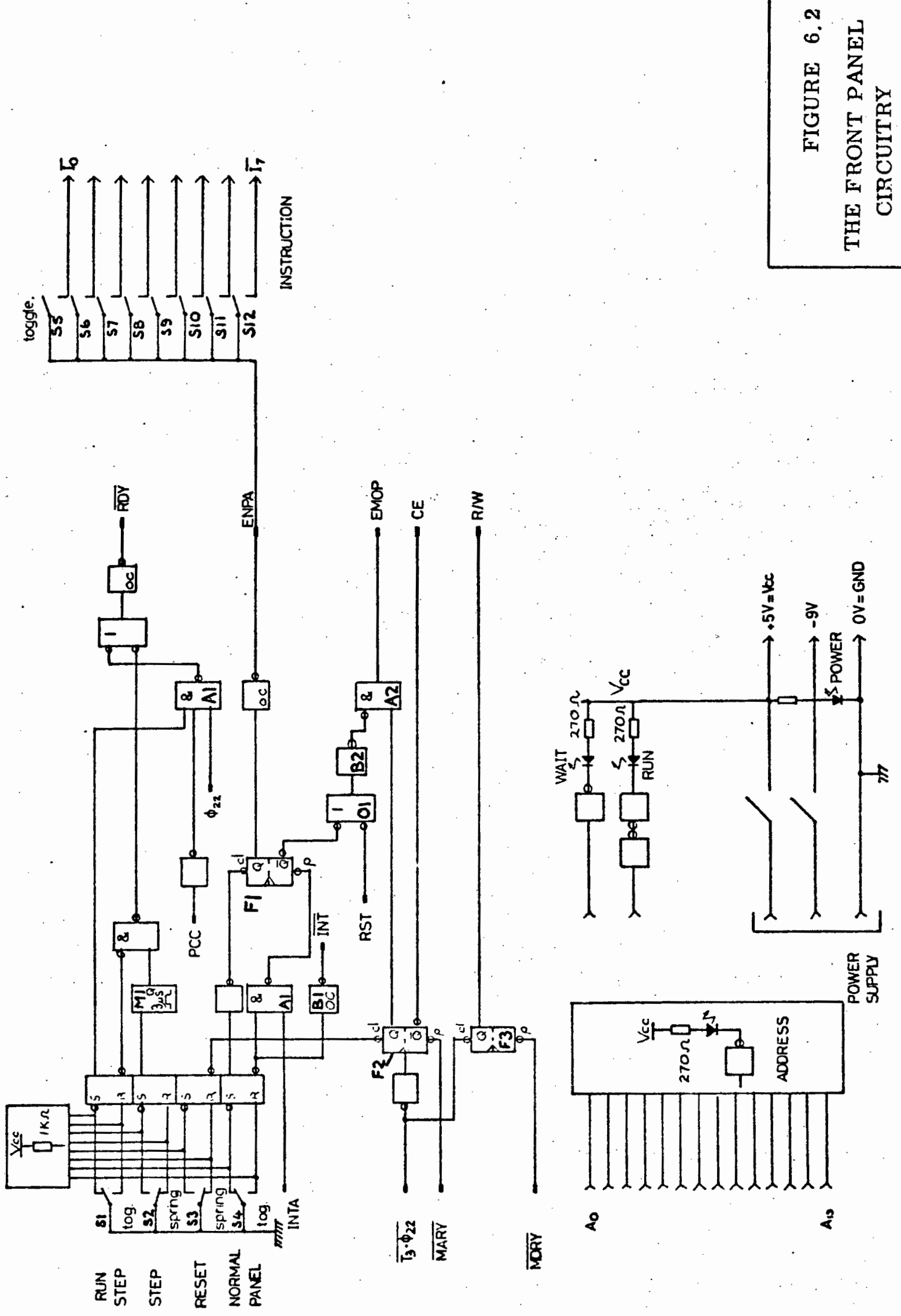
(Chapter 6 - continued)

The toggle switch, S4, performs the following functions:

(i) In the normal mode, it holds the $Q$ output of flip-flop F1 in a high state, and disables the "INSTRUCTION" input switches from the I-bus. The I-bus is now free to receive signals from memory when required because the signal EMOP (enable memory output) will be in the true state.

(ii) If $\mathrm{S} 4$ is now switched to the panel mode, the following sequence of events takes place.

(1) The "CLEAR" is removed from F1.

(2) An intermpt is generated and sent to the 8008 via buffer B2.

(3) When the 8008 acknowledges this interrupt (INTA), the flip-flop, F1, is preset via gate Al.

(4) EMOP is disabled (gates $01, B 2$ and A2).

(5) The 8008 will now receive its next

instruction from the switches S5 - S12.

As the output of buffer $B 1$ remains in a low state, all further interrupts are inhibited while $S 4$ remains in the panel mode.

RST is a signal which can disable EMDP if an interrupt occurs during normal program execution while $\mathrm{S} 4$ is in the normal mode. This is in order to "jam in" an alternate interrupt instruction, (see Chapter 6. 8 ), during normal program execution.

The switch, S4, can also be used to start the processor after power on, or to re-start the processor after it has entered a "stopped-state".

The processor can be made to begin program execution from any desired memory location, as follows:

(i) Start with the switch positions as in Figure 6.2.

(ii) Switch S1 to position "STEP".

(iii) Press reset button (switch S3)

(iv) Switch S4 to position "PANEL". 
(Chapter 6 - continued)

(v) Enter a jump instruction via the "INSTRUCTION" switcies (01000100)

(vi) Press "STEP" (switch 22 )

(vii) Enter first byte of execution address via "INSTRUCTION" switches $(A O-A 7)$

(viii) Press "STEP"

(ix) Enter second byte of execution address via "INSTRUCTION" switches (A8 - A14).

(x) Press "STEP".

(xi) The desired execution address should now be displayed on the "ADDRESS" light-emitting diode displays. Switch S4 to "NORMAL" and "is S1 to RUN: the processor will then execute the desired program.

S1 to RUN will cause the processor to execute the desired program.

By switching S4 to "NORMAL", and alternately pressing the "STEP" switch, the operator can execute the program, one byte at a time.

By switching S4 to "PANEL" and SI to "RUN", the processor will repeatedly attempt to execute the instruction set on the "INSTRUCTION" switches. If a one-byte instruction is used, the processor will continuously output all the possible memory addresses on its Address bus, $A_{0}-A_{13}$ :

The two flip-flops, F2 and F3, are used to supply the timing relationships required to interface the 8008 with its memory. (See Chapter 6.3)

\subsection{THE MEMORY}

Slots three and four of the processor rack are reserved for memory. In the prototype, only slot four is used and contains eight kilowords of eight-bit semi-conductor memory. This memory module consists entirely of 1024 × 1 bit Static MOS random access memory chips, along with the relevant decoding circuitry. The Inte1 2102 memory element is used and full manufacturer's data for this component appears

$$
\text { in/... . }
$$


(Chapter 6 - continued)

in Appendix 3.

The decoding circuits and memory organization have been chosen to ensure easy interleaving of one kiloword pages of random access or read-only memory as required.

Figure 6.3 shows the decoding circuits and the memory organization. Note that the ten low-order bits of the address are connected in parallel to the ten address-selection inputs of all the 2101 's. The four high-order bits $\left(A_{10}-A_{13}\right)$ are fed to a four-to-sixteen line decoder, D1, which selects any one of sixteen possible memory pages of one kiloword each. By interchanging the outputs of $\mathrm{D} 1$, different types of memory can be interleaved in one kiloword page increments. Note that each selected output of D1 goes to the chip select (CE) inputs of eight 2102 's making up a memory page. The read/write $(R / W)$ inputs of all the memory chips are connected together. When this signal is held in a low state, the memory is prepared for a write cycle. With the R/W signal in a high state, a read cycle is implemented.

The internal arrangement of one memory page using eight 2102 's is shown in figure 6.4. Note that all the data-out and data-in pin connections for each bit position (i.e. lowest order bit) of each page are connected together.

\subsection{INPUT-OUTPUT DEVICE ADDRESS DECODING:}

There are two decoders in the processor which determine the address of the device to which the programmer requires access. The one decoder is located on card two and serves that particular card. The other decoder is on card five and similarly serves the devices specified on card five.

The two $I / 0$ instructions of the 8008 are:

(1) For input : 0100MMI

(2) For output : OIRRMMI (RR $\neq \infty)$

It can be seen that different combinations of MMM and RR will permit a maximum of 8 input ports and 24 output ports. During input/output operations 


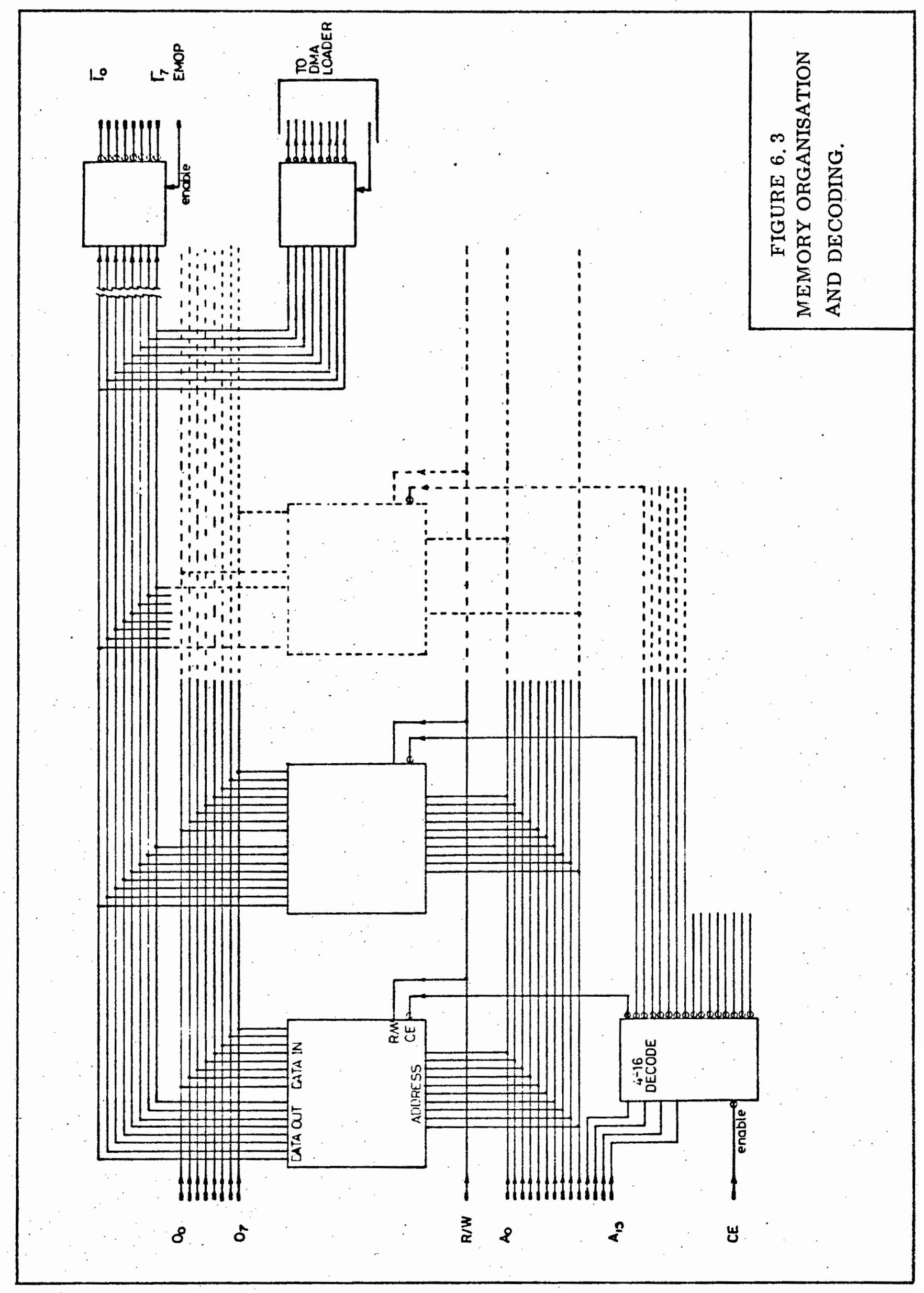




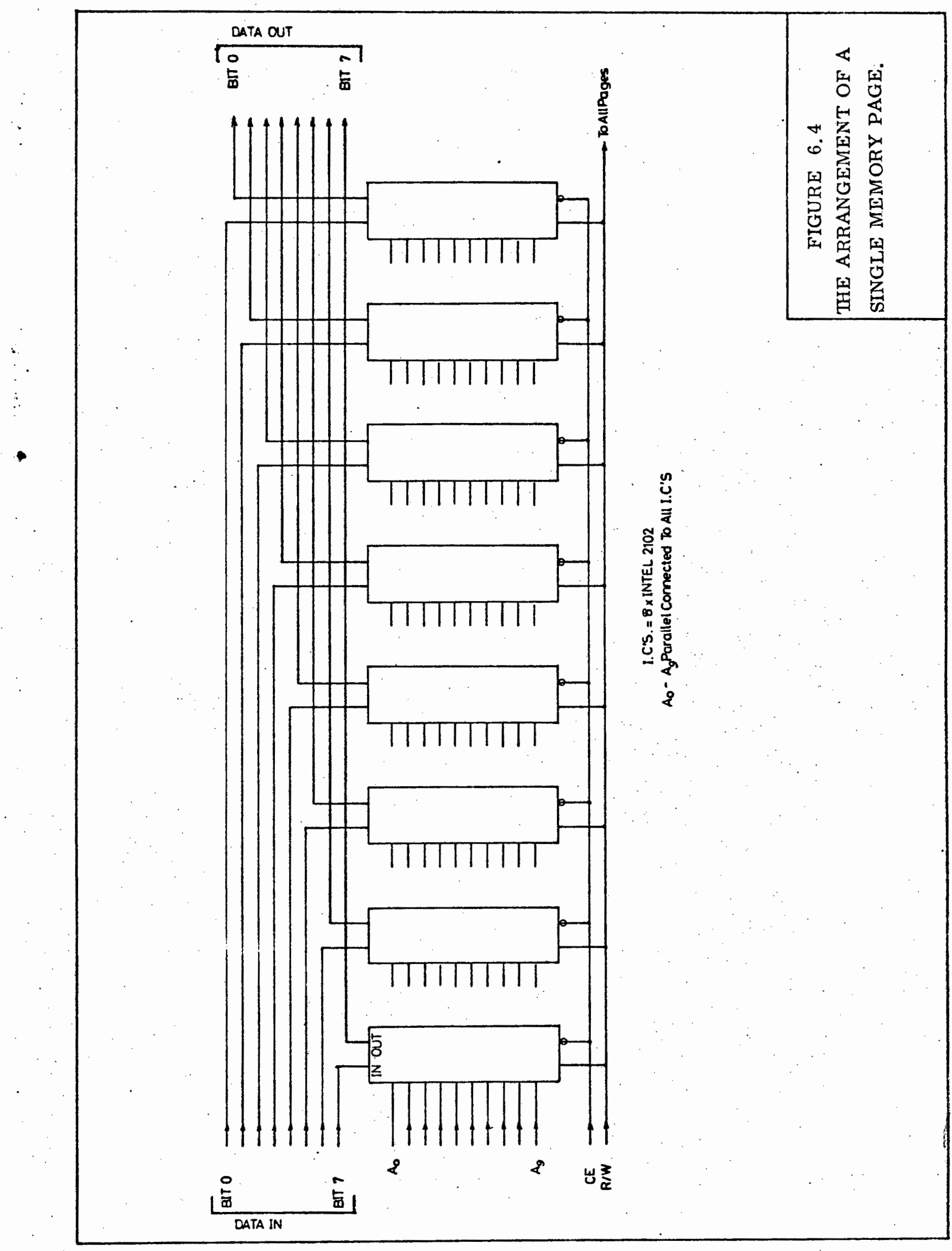


(Chapter 6 - continued)

the lower order six bits of these instructions are made available during the T2-state on the address lines $A_{8}-A_{13}$.

Figure 6.5 shows the circuitry of the decoders. I/O DECODER 1 serves card two and I/O DECODER 2 serves card five. Table 6.1 shows all possible combinations of the input/output instructions, along with their octal equivalents and how they are utilized. All further references to these instructions will be in octal form.

(a) I/O DECODER 1:

From Table 6.1 it can be seen that this decoder detects four input $(111,113,115,117)$ and four output $(131,133,135,137)$ instructions.

These eight instructions can be uniquely distinguished from the others by the fact that the signal, $A_{13}$, is always low and the signal, ${ }_{11}$, is always high. Therefore the signal $\overline{\mathrm{PCC}} \cdot \mathrm{W} \cdot \mathrm{A}_{\|} \overline{\mathrm{SYNC}} \cdot \overline{\mathrm{A} 13}$ (gates Al and A2) is used to enable the decoder D1 which selects one of these eight I/O instructions according to the state of the signals $A_{12}, A_{10}$, and $A_{9}$. When any of the instructions, 135, 133, 131 or 111, have been decoded for the 8008 , it is not necessary to "wait" for the $\overline{\mathrm{RDY}}$ signal to be generated (Gates 01 and $A 3$ ).

(b) I/O DECODER $\cdot 2$ :

This decoder detects four input $(101,103,105,107)$ and twelve output $(121,123,125,127,141,143,145,147,161,163,165,167)$ instructions.

These sixteen instructions can be distinguished from the others by the fact that the signal $A_{11}$ is always low when such instructions occur. Therefore the signal $\overline{\mathrm{PCC}} \cdot \mathrm{W} \cdot \overline{\mathrm{A}}_{11} \cdot \overline{\mathrm{SYNC}}$ (gate $\mathrm{A}^{4}$ ) is used to enable the decoder which selects one of these sixteen $I / O$ instructions.

As none of these instructions requires the 8008 to enter a "wait" state, $\overline{\mathrm{RDY}}$ signals are generated by gate $\mathrm{A} 5$ when they are used. 


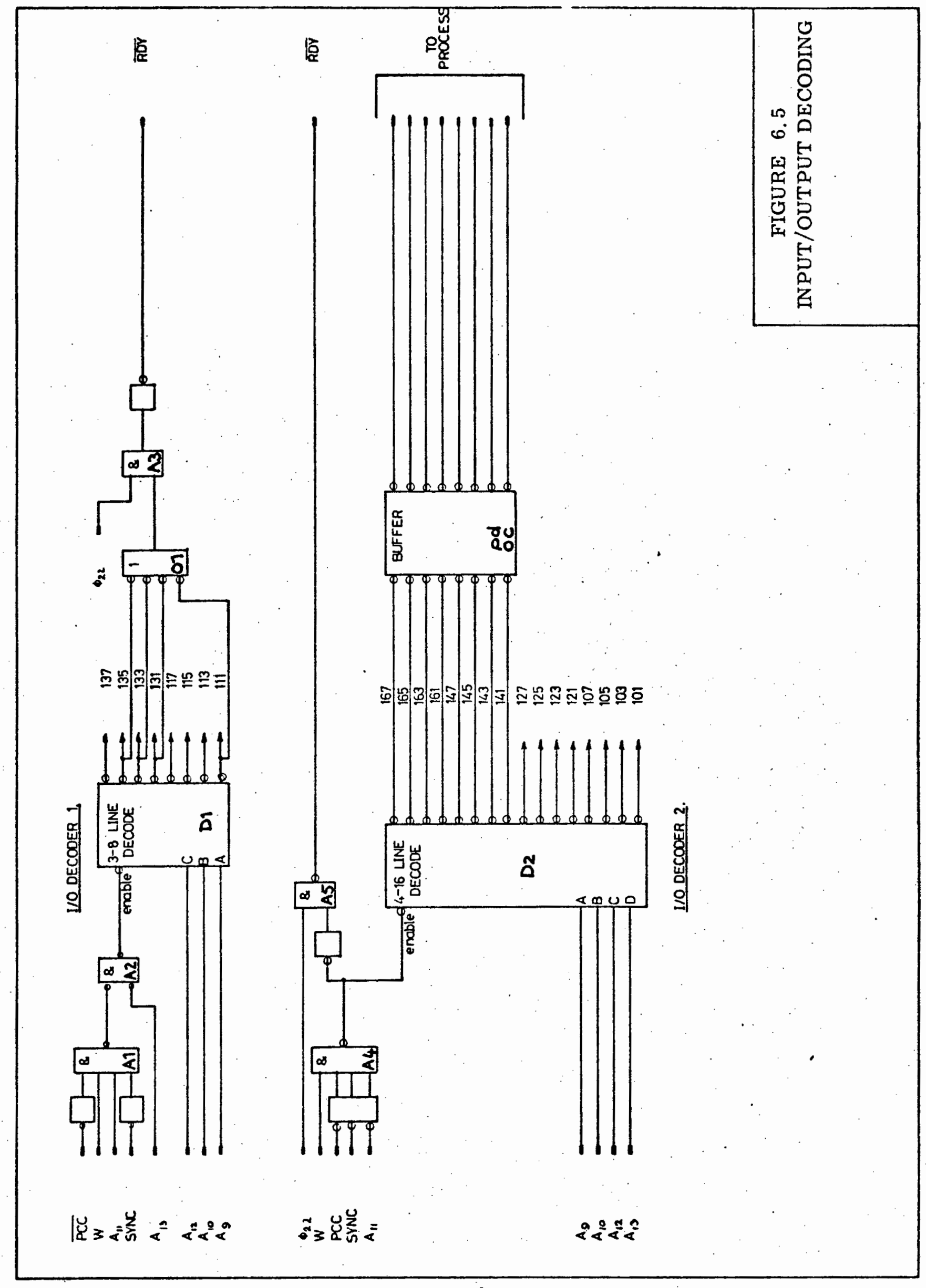




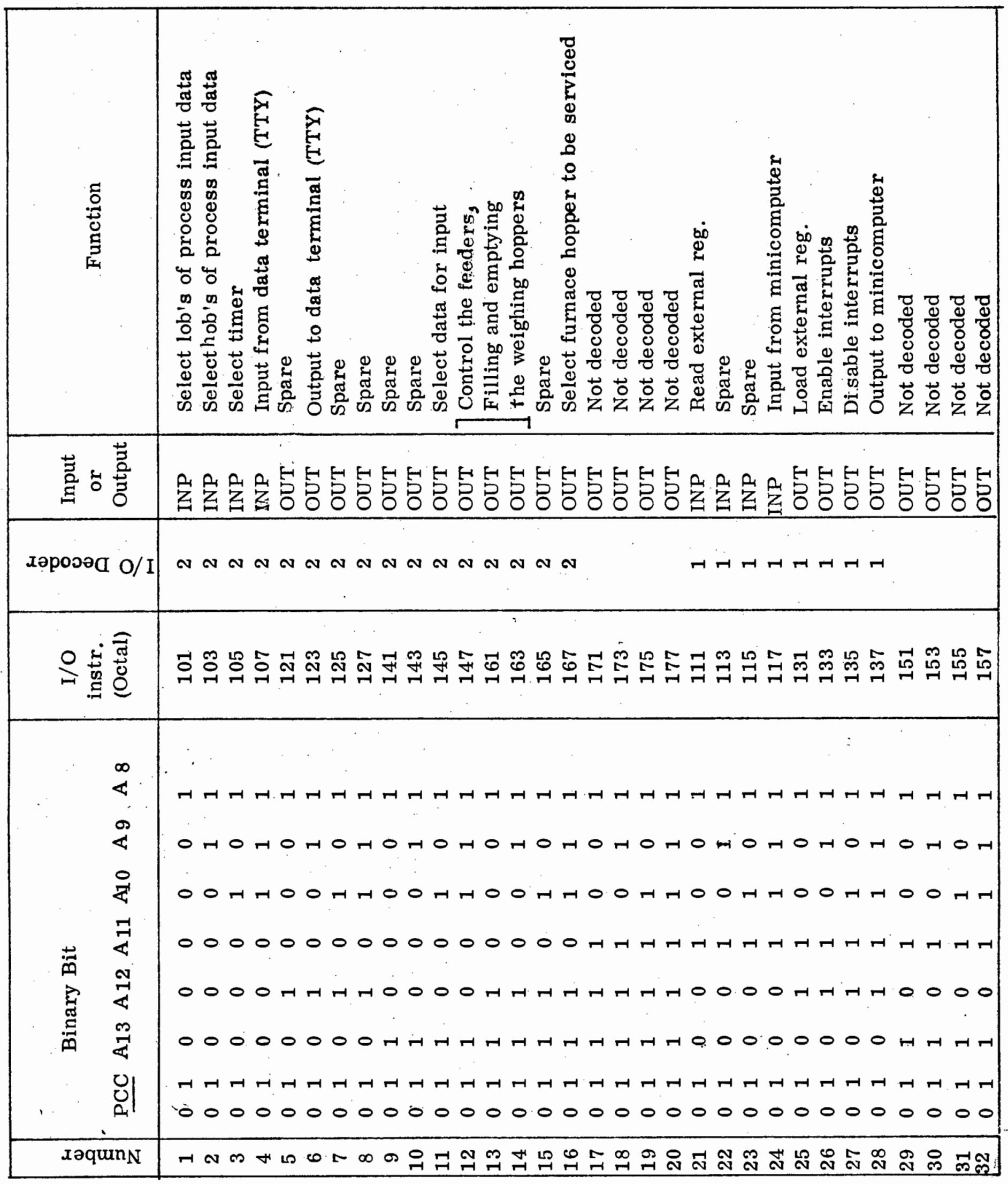


(Chapter 6 - continued)

\subsection{INTERFACING TO A MINICOMPUTER}

As previously mentioned, interfacing to a minicomputer is implemented using two interfaces. An interface on board two is designed to accept signals from, and send signals to, a minicomputer. These signals are fully decoded, and it was ensured that they could easily be interfaced. A further interface is required to adapt these signals to the particular minicomputer being used - in this case a VARIAN 620/L - 100 .

These interfaces serve two functions:

(i) During program development: the programmer can use a minicomputer directly to load the microcomputer memory. A Direct Memory Access channel is used for this operation.

(ii) In a distributed control system: this link has been provided so that the two computer systems can pass data between each other under program control.

Figure 6.6 shows the interfacing associated with the processor. Similarly Figure 6.7 shows the circuitry needed to interface the processor to a VARIAN $620 / \mathrm{L}-100$.

Note: As the VARIAN was used only for program development on the prototype, no attempt was made to interlace the prototype into the VARIAN'S priority interrupt chain.

(a) To exchange data under program control

This sequence is normally initiated by the minicomputer executing an external control command, an "EXC' instruction for the VARIAN: If the processor needs to initiate such a data exchange, it must issue an interrupt signal to the minicomputer. The resulting interrupt service routine causes the minicomputer to input a status word supplied by the processor, thus obtaining information about the processor's requirements. 


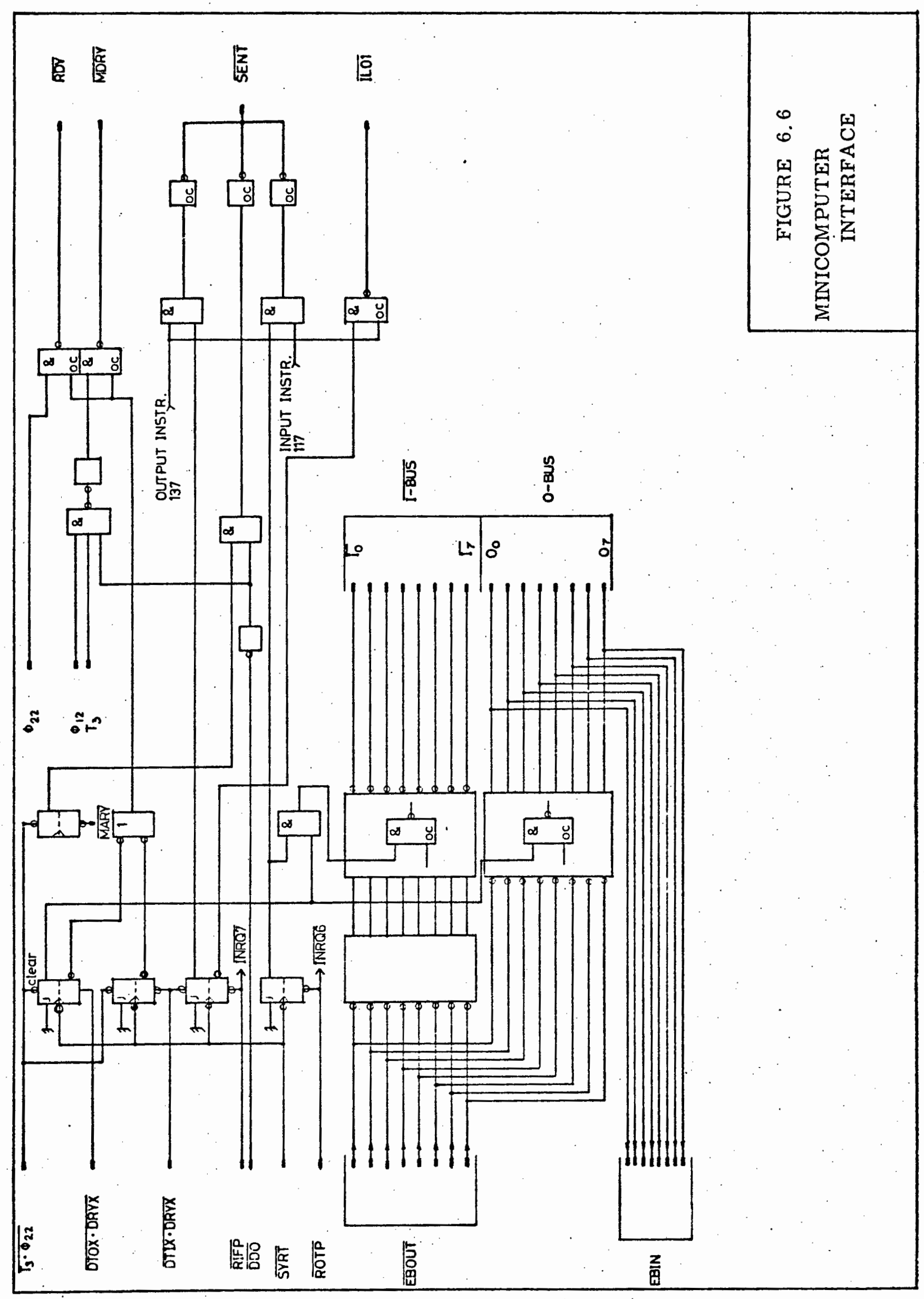




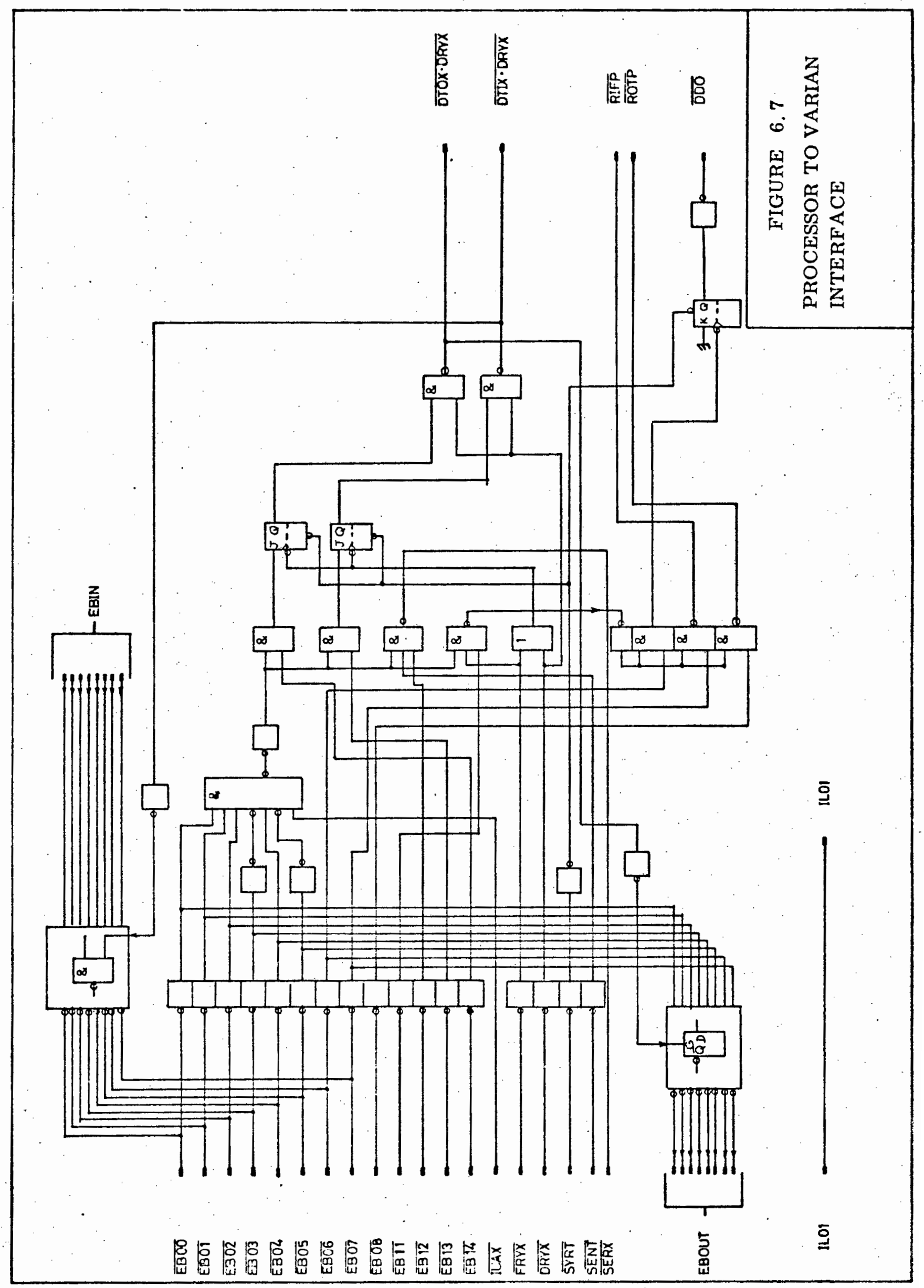


(Chapter 6 - continued)

(i) To send data from the VARIAN to the processor:

An EXC command $\left(100427_{\delta}\right)$ is decoded and sent to the processor $\overline{(\mathrm{ROTP})}$. The VARIAN then enters into a "sense 10op", waiting to output data to device number 27 , which is the processor. In the meantime, the signal $\overline{\mathrm{ROTP}}$ causes the processor to be interrupted. The processor's interrupt service subroutine then initiates data input using input instruction 117. This instruction is decoded by I/O DECODER 1 , and a "sense true" signal is sent back to the VARIAN. In receipt of this "sense true" signal, the VARIAN outputs the required data during the signal $\overline{\mathrm{DTOX}} . \overline{\mathrm{DRYX}}$ (data transfer out and data phase of computer cycle). This signal also produces the necessary "ready" pulse to return the processor from its "wait" state.

(ii) To send data from the Processor to the VARIAN:

A similar process to (i) above is used, except for the following points:

$\therefore$ EXC instruction $=1002278$

- Decoded signal to processor $=\overline{\mathrm{KIFP}}$

- Processor output instruction $=137_{8}$

- VARIAN data input signal $=\overline{\text { DTIX.DRYX }}$

(b) Loading the Processor Memory using Direct Memory Access from a Minicomputer:

This sequence is entirely controlled from the VARIAN. The VARIAN expects the processor to be in a "wait" state, with its memory address register containing the address of the lowest memory location to be loaded. The processor's front panel switches must be set for "step" and "panel" modes. The instruction switches must contain a "no-operation" instruction code, i.e. $300_{8}$.

The sequence is initiated by the execution of an external control command, EXC $100127_{8}$, by the VARIAN. This sets a flip-flop which, via signals $D D O$ and $O B I N$, disables the microprocessor's data output register. 
Any data sent from the VARIAN is loaded into the chosen processor memory location and a "ready" pulse is supplied to the processor. The processor then executes the "no-operation" instruction and increments its address register. This will then hold the address of the next memory location.

Thus the processor's program counter is used to generate the memory addresses to be loaded. Consecutive memory locations of the processor are loaded by repeatedly sending the required data from the VARIAN.

\subsection{THE DIRECT MEMORY ACCESS LOADER AND DISPLAY}

This device was designed as a software development aid. It consists of the following ( see Figure 6.8):

- Eight light-emitting diodes (1ed 1-8)

- Eight Octal push-button switches (SO-S7)

- A toggle switch which can be in either the "examine" or "load" modes.

- A push-button switch to clear the current entry displayed on the led's. 1- 8 .

- A push-button switch to load the current entry into the current memory location.

- The necessary control circuits for the above.

The memory loader has the following functions:

- To display the contents of the current memory location while "stepping" through a program (examine mode).

- To load the current memory location with the data displayed while the processor is in the "panel-mode".

Note: The address register is automatically incremented in a similar way as for a Direct Memory Access load from a minicomputer.

The data is entered in OCTAL ( 3 digits) and decoded into binary by a diode Read Only Memory. The binary information is shifted along a shift register consisting of Lrtype flip-flops, and is loaded into the current memory location when the "load" button is pressed.

At present, the Direct Memory Access loader is contained in a separate box but could, with value, be included on the front panel. 


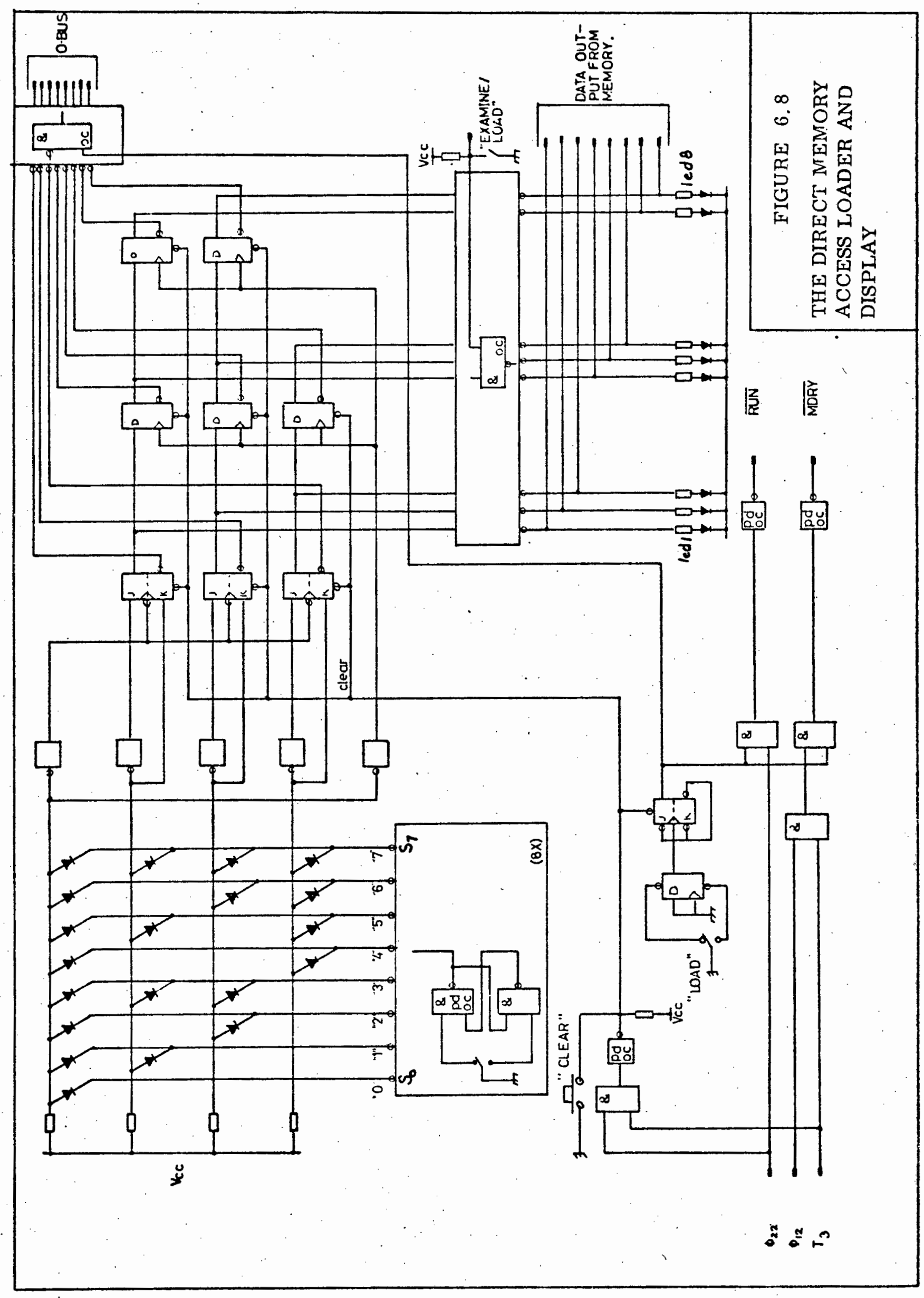


(Chapter 6 - continued)

\subsection{EXTERNAL HARDWARE REGISTERS}

As discussed in Clapter 5.4, a major disadvantage of the 8008 microprocessor is the addressing scheme enpioyed which requires memory addresses to be held in a specific register pair. Therefore, two external hardware registers have been added into which the programmer can deposit the contents of the A-register and any other register. This will then leave two registers free into which the current address can be temporarily stored during an interrupt servicing routine.

The circuitry of these two registers is built on board 2, and is shown in Figure 6.9. They consist essentially of two Intel integrated circuits which are 8-bit latches. (See Appendix 4) Output instruction $13 I_{8}$ causes the current contents of the A-register to be sent to one of these registers. Input instruction $111_{8}$ causes the A-register to be loaded from one of these registers.

After each communication with any one of the registers, flip-flop F1 toggles, causing a pointer to select the other register for the next imput or output operation.

Chapter 11.9 gives an example of how the progranmer may use these registers during an interrupt sequence.

\subsection{THE INTERRUPT CONTROLLER}

The intermpt controller is also built onto card two. Its circuitry is shown on figure 6.10. The interrupt controller features

- a software enable/disable facility

- an interrupt priority scheme

- the ability to intermpt $\wedge$ anyone of eight addresses by using the RST instruction (OOAAA101)

- the ability to store all interrupt requests until they are serviced. 


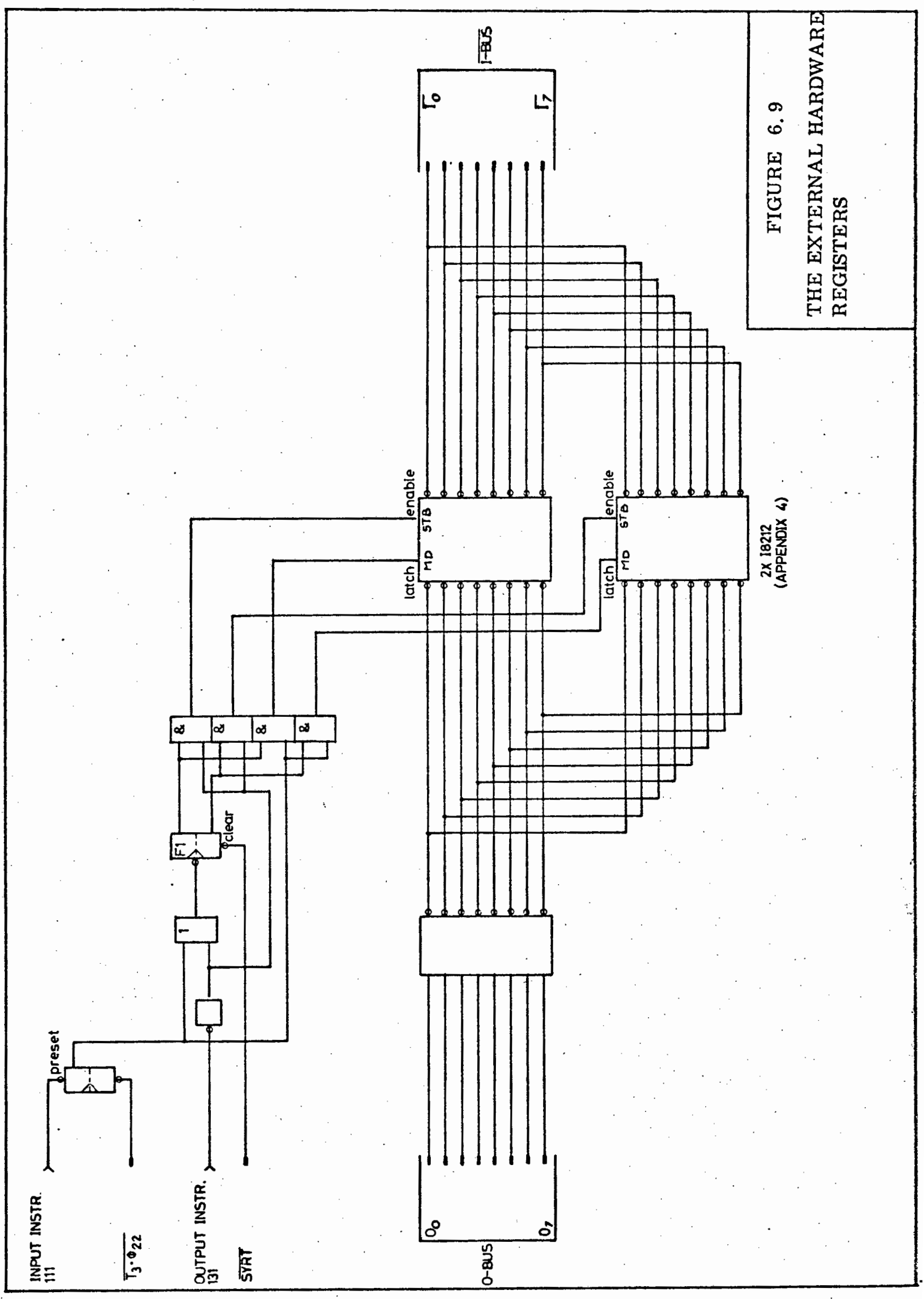




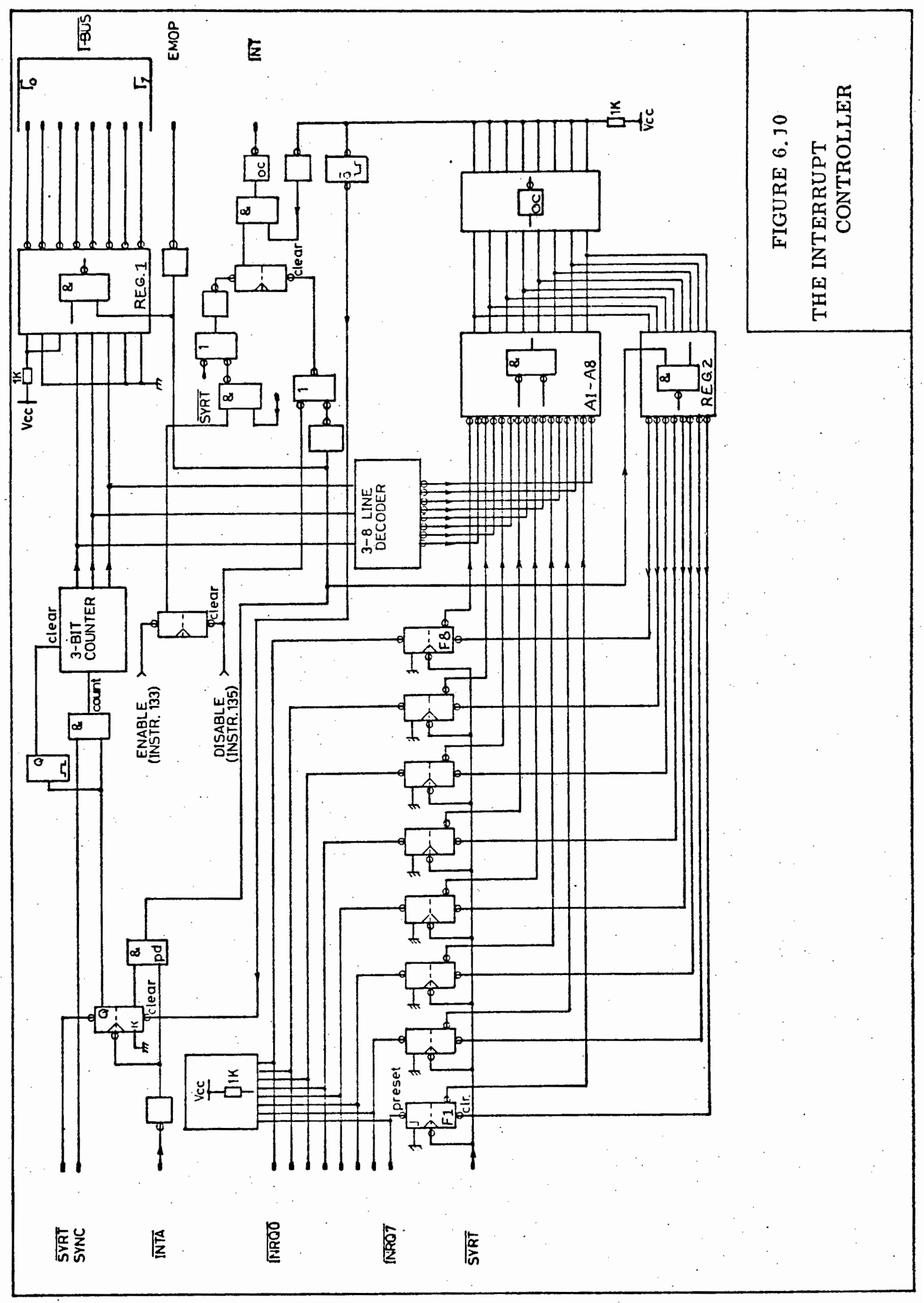


(Chapter 6 - continued)

An interrupt request will cause one of the flip-flops F1 - F8 to be pre-set, thus causing the flip-flop complement output to go to a low state. $\mathrm{Cl}$, a synchronous three-bit counter feeds a 3-8 line decoder, which alternately tests each one of the F1-F8 flip-flop's outputs. When any of these outputs is in a low state, this condition will be detected by one of gates $\mathrm{Al}-\mathrm{A} 8$. The outputs of $\mathrm{Al}-\mathrm{A} 8$ are dot-ored together, thus generating an interrupt to the 8008 microprocessor, and stopping the, counter CI with the address of the interrupting device on its output. These three bits then form part (AAA) of the RST instruction which is placed on to the processor's input via Register $I$ at the time when the processor acknowledges

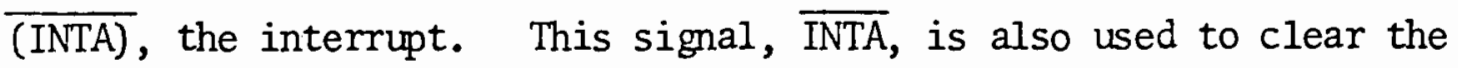
appropriate flip-flop, using REG. 2. The counter is also reset via monostable, MI.

The interrupts are assigned as follows:

$$
\text { ( } \overline{\text { INRQO }}=\text { highest priority) }
$$

\begin{tabular}{|c|c|c|c|}
\hline INTERRUPT VECTOR & RST (OCTAL) & $\begin{array}{l}\text { INTERRUPT } \\
\text { ADDRESS } \\
\text { (OCTAL) }\end{array}$ & FUNCTION \\
\hline$\overline{\text { INRQO }}$ & 005 & 00000 & To start the processor \\
\hline$\overline{\text { INRQ1 }}$ & 015 & 00010 & \\
\hline$\overline{\text { INRQ2 }}$ & 025 & $00020^{\circ}$ & For use by the process \\
\hline$\overline{\mathrm{INQR3}}$ & 035 & $\infty 0030$ & \\
\hline$\overline{\mathrm{INQR4}}$ & 045 & 00040 & Data to Teletype \\
\hline$\overline{\mathrm{INQR5}}$ & 055 & 00050 & Data from Teletype \\
\hline$\overline{\mathrm{INQR6}}$ & 065 & 00060 & Data from minicomputer \\
\hline$\overline{\text { INQR7 }}$ & 075 & 00070 & Data to minicomputer \\
\hline
\end{tabular}

Note: For power failure re-start, a different interrupt mechanism is to be used, as discussed in Chapter 7. 
(Chapter 6 - continued)

Figure 6.11 shows circuitry of the teletype interface. The output (parallel to serial) and input (serial to prarllel) shifts registers are shown in Figure 6.12. All these circuits are located on board 5 .

The interface is designed for a teletype having a twenty milliamp signalling current and a forty volt battery. After each input or output operation is completed, the interface interrupts the processor indicating that the teletype is ready for the next operation.

6.10 THE TIMER AND COMUNICATION WITH THE PROCESS

Figure 6.13 shows these circuits.

(a) The Timer: This consists of a $1 \mathrm{~Hz}$ clock fed intọ a $\div 256$ counter. Input instruction 105 causes the output of the counter to be placed on the microprocessor's data input bus via register REG. 1 .

(b) Data Input to the Processor: This is a sixteen-bit bus (DIBO to DIB15) which can be placed onto the microprocessor's data input bus in one of two eight-bit bytes (REG. 2 and REG. 3). Input instruction 101 is used for the low-order bits and instruction 103 is used for the high-order bits. (c) Data Output to the Process: As shown in Chapter 6.4, eight output instructions are decoded by I/O DECODER 2 and are available to the process. The microprocessor's data-output (O-BUS) is buffered by B1 and is available to the process.

These output signals may be used as follows:

(i) To send an eight-bit byte to any one of eight destinations.

(ii) To select one of eight sixteen-bit data words which are to be placed on the data input bus (DIBO-D1B15).

(iii) Each eight-bit data output bus can be decoded into one of two hundred and fifty-six output control pulses.

(iv) Each eight-bit data bus can be decoded to select any one of two hundred and fifty-six, sixteen-bit words of data to be placed on DIBO-D1B15. 


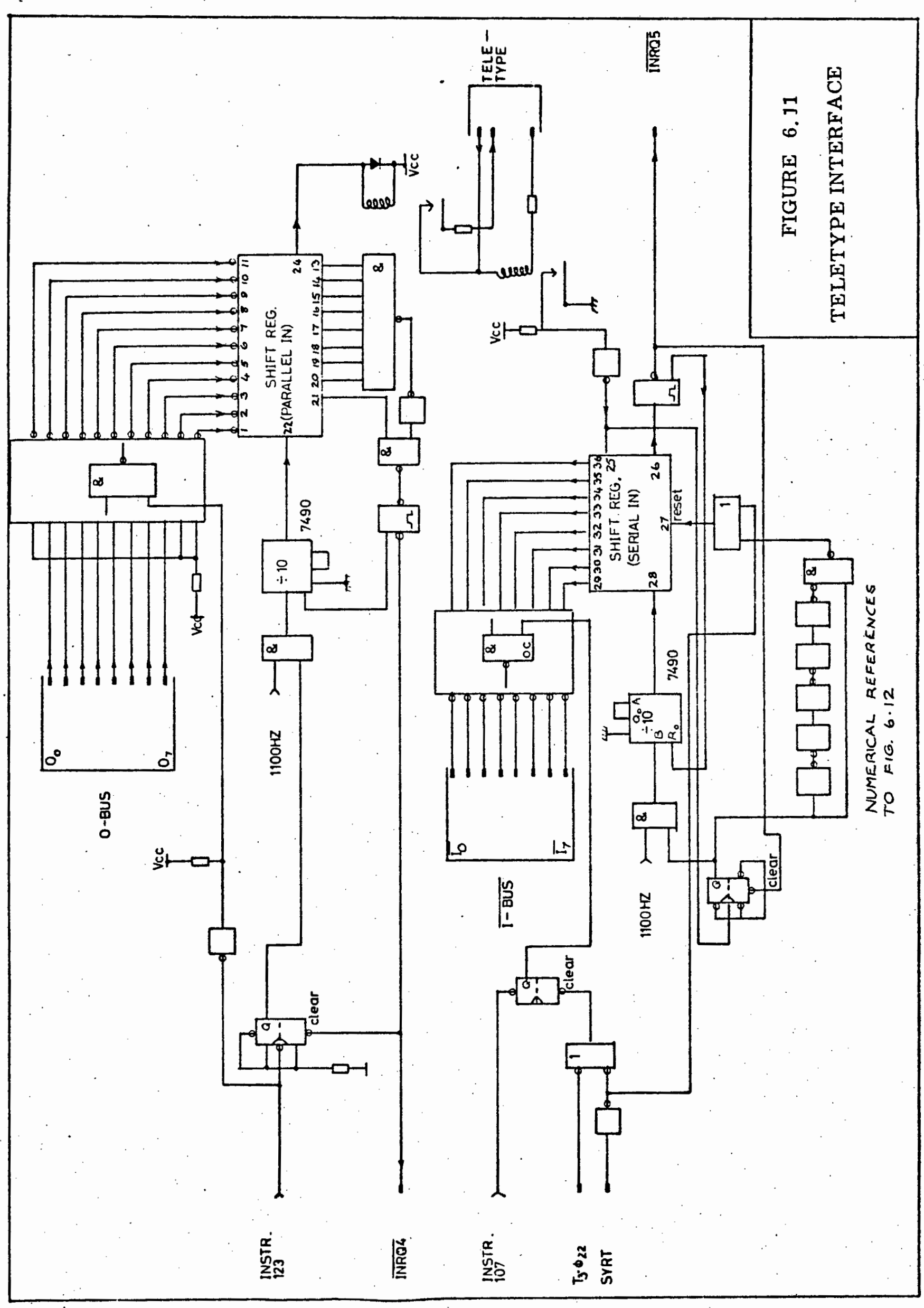




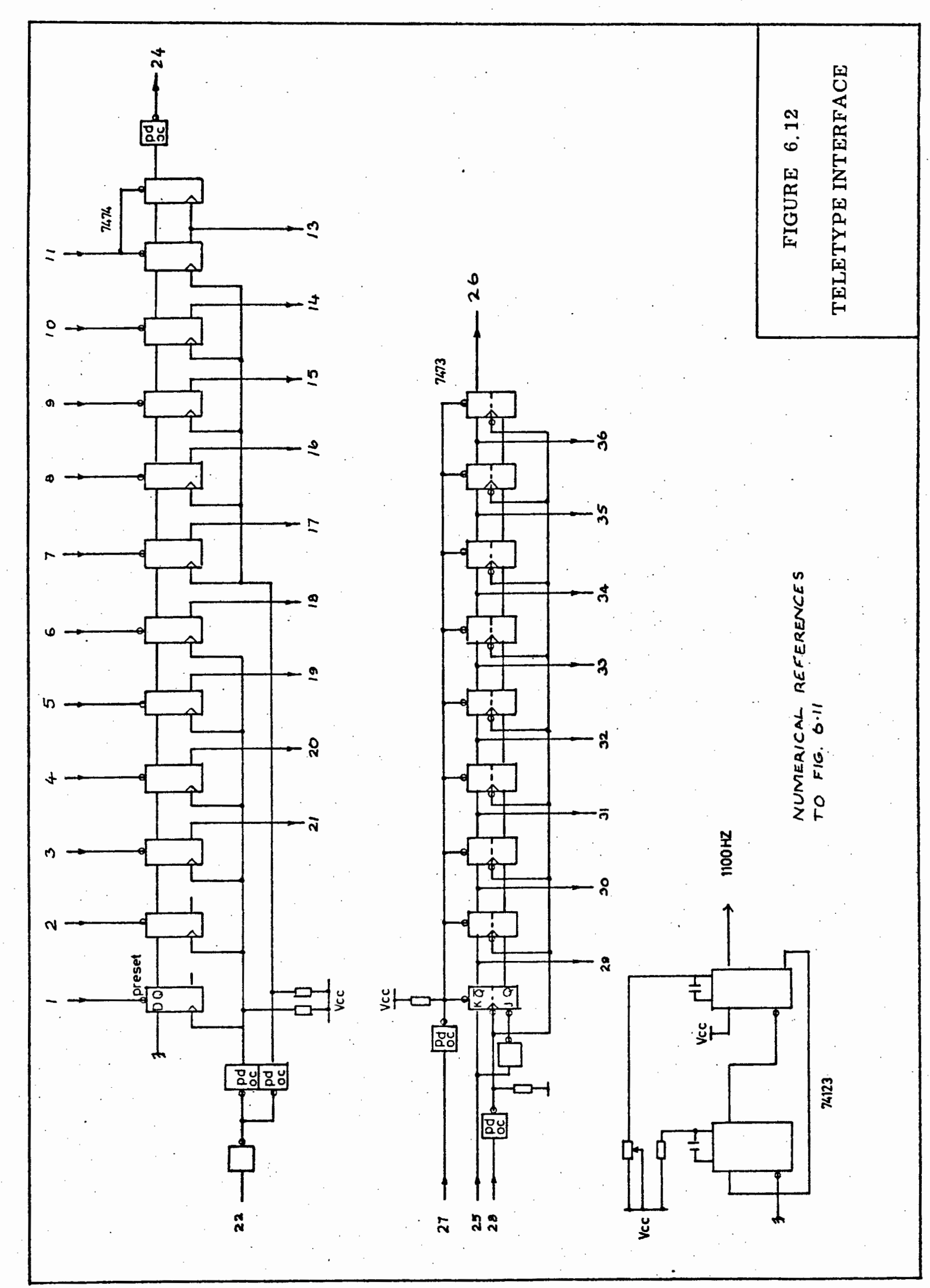




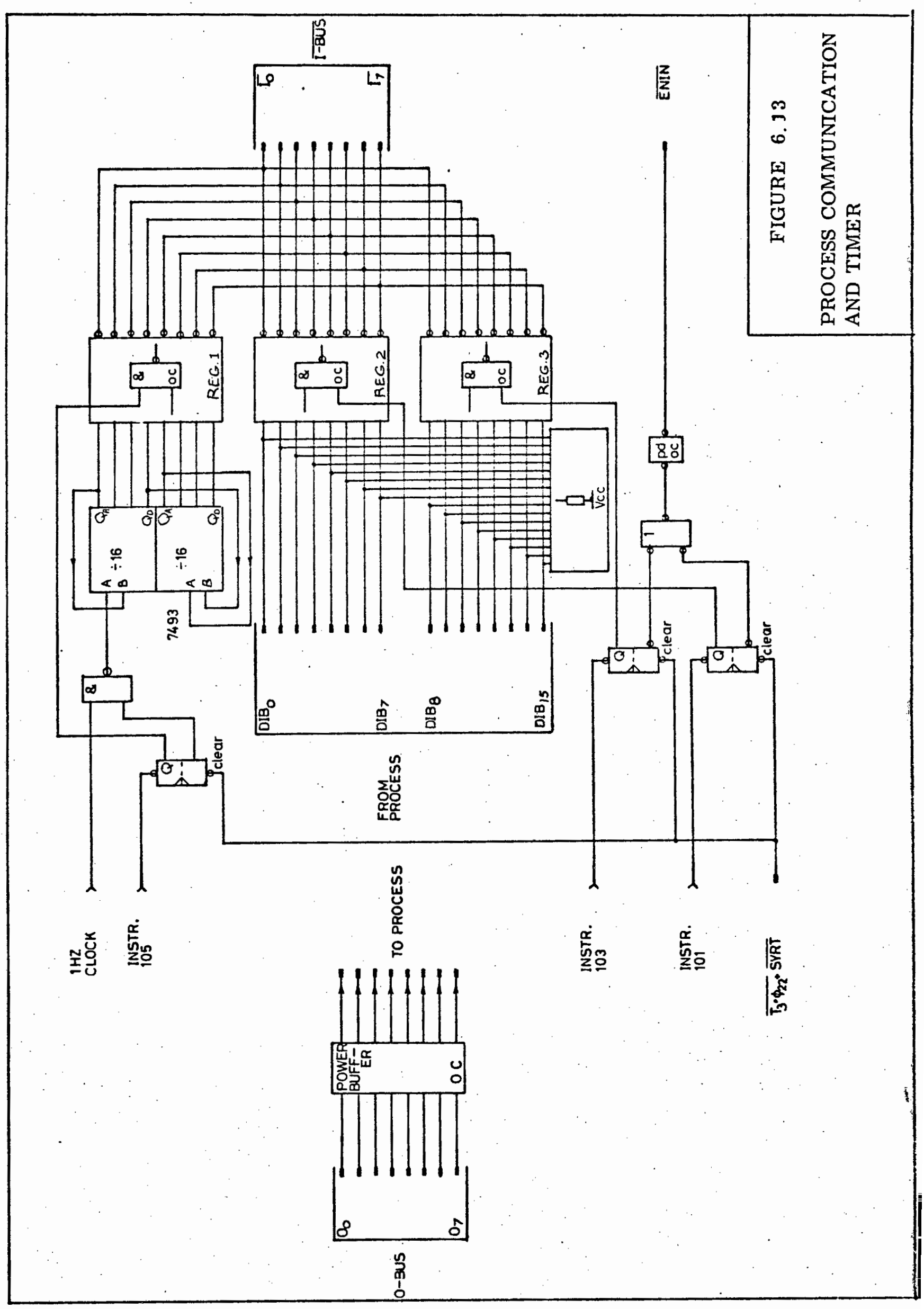


(Chapter 6 - continued)

(v) Combinations of the above are possible.

Note: That whenever the processor inputs from D1BO-D1B15, a signal

$\overline{\text { ENIN }}$ is made available to the process. $\overline{\text { ENIN }}$ enables the designer to multiplex inter-process signal onto D1BO-D1B15. Whenever the signal, $\overline{\mathrm{ENIN}}$, is in a true state, the bus should be freed for use by the processor. 


\section{CHAPTER $\cdots 7$}

\section{POWER AND PONER FAILURE}

An investigation was undertaken into the power standby requirements of intelligent controllers during a power failure. This, along with the specific needs of the processor, revealed the following points:

- The semi-conductor random access memory which will be used for variable and inventory storage is destructible during a power failure.

- It is desirable that the inventory information is protected for at least one shift period (eight hours). Any power failure longer than this is regarded as being so catastrophic that the loss of inventory information is of relatively minor importance.

- The processor's power failure software will require approximately ten milliseconds in order to effect a program-controlled shut-down after the power failure is first detected.

- The processor will need to know the extent of the power failure, i.e. which parts of the process are affected, and so on.

- The process has an under-voltage trip mechanism which shuts off the motors driving the feeders, belts, and so on. This trip mechanism has to be reset when the power supply is restored.

Experiments were carried out to determine a suitable power supply system. As a result of this, the power system shown in Figure 7.1 was devised.

Note: The processor is first notified of the power failure by a special interrupt which cannot be disabled (Figure 7.1). ${ }_{\text {and }}$ The processor can also monitor the state of the power supply system via $\Lambda_{\Lambda}$ input port.

Sugges ted circuits for a power failure interrupt generation are shown in Figure 7.2. This interrupter "jams" in three, successive bytes of a jump instruction, thus causing the program to transfer control directly to the power failure routine. 


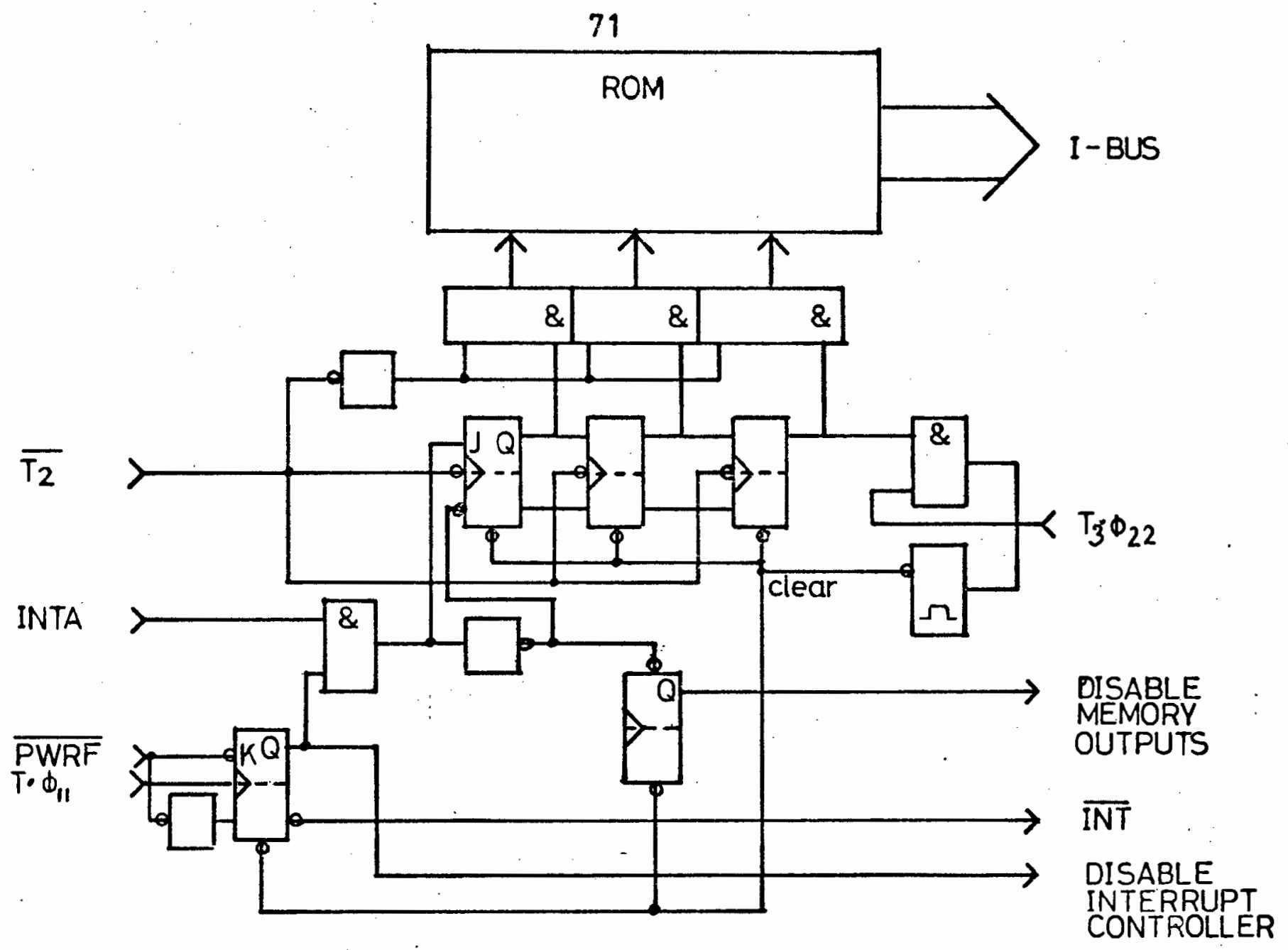

FIGURE 7.2 SUGGESTED POWER FAILURE INTERRUPTER

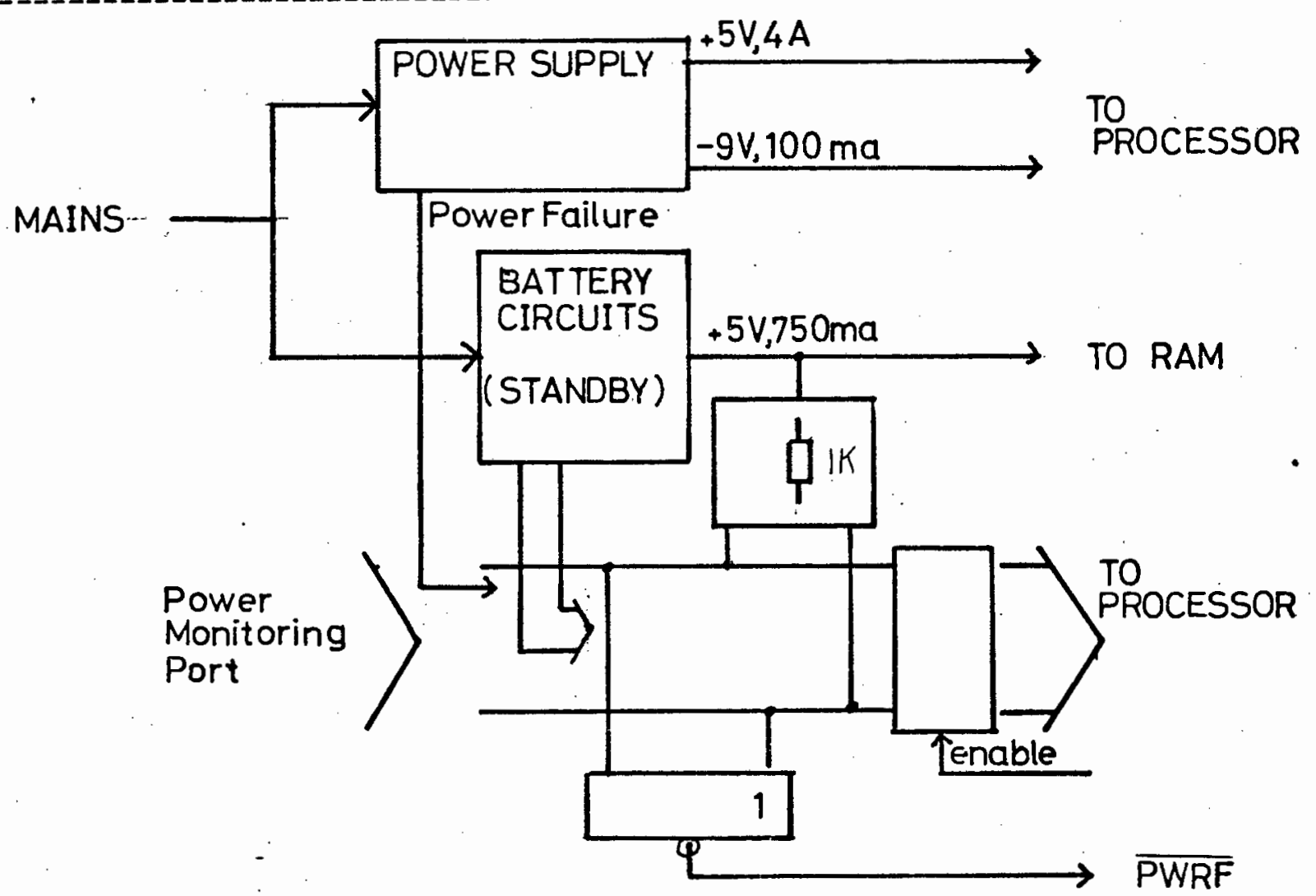

FIGURE 7.1 SUGGESTED POWER SUPPLY SYSTEM 
(Chapter 7 - continued)

When a power failure occurs, the line PWRF goes to a low state, causing the processor to enter its power failure routine. The current contents of all the registers are stored and the processor "reads" the power failure monitoring port. If the power failure is only momentary, none of these lines will be in a low state and the processor can return to its previous task: otherwise, suitable action can be taken. During a power failure program, a flag in memory should be set so that a normal "power-on" can be distinguished from a "power-on" after a power failure. In each case, the processor will start from address 0000 . 


\section{QHAPTER 8}

\section{THE PROCESS INTERFACE}

\subsection{INTRODUCTION}

Chapter 6.10 discusses hardware facilities which are provided by the processor for process control functions. This chapter will deal with how these facilities have been utilized to control the batch weighing and transport system simulator.

On the prototype the process interface is situated in the simulator cabinet and directly inter-connected to the simulator. On a real plant this interface would be built into the converter cabinet where the necessary signal conditioning would also take place. The systems designer would also have to ensure that the necessary signal levels are obtained and also the necessary degree of isolation between the processor and the process is maintained.

\subsection{THE INTERFACE}

Refer to Figure 8.1. An appropriate output instruction causes the contents of the A-register to be output on the O-bus $\left(O_{0}-O_{7}\right)$ and stored in one of six registers (REG 1 - REG 6). The first four bits of REG 1 are decoded by $\mathrm{D} 1$ into one of sixteen signals which will be used to control the conveyor and shuttle system so that a route is established to the selected furnace hopper.

REG 3 sets the weighing hopper mode, i.e. whether it is being filled in the coarse or fine feed mode, or whether it is being emptied.

REG 4 and 5 control the vibration feeders. The feeders are either in the stopped or running modes according to the contents of REG 4 and 5

REG 6 selects one byte of data (maximum 16-bits) which is to be placed on the processor's data input bus (DIB). Decoder, D2, selects general input ports which enable the processor to monitor the process. Decoder, D3, selects 


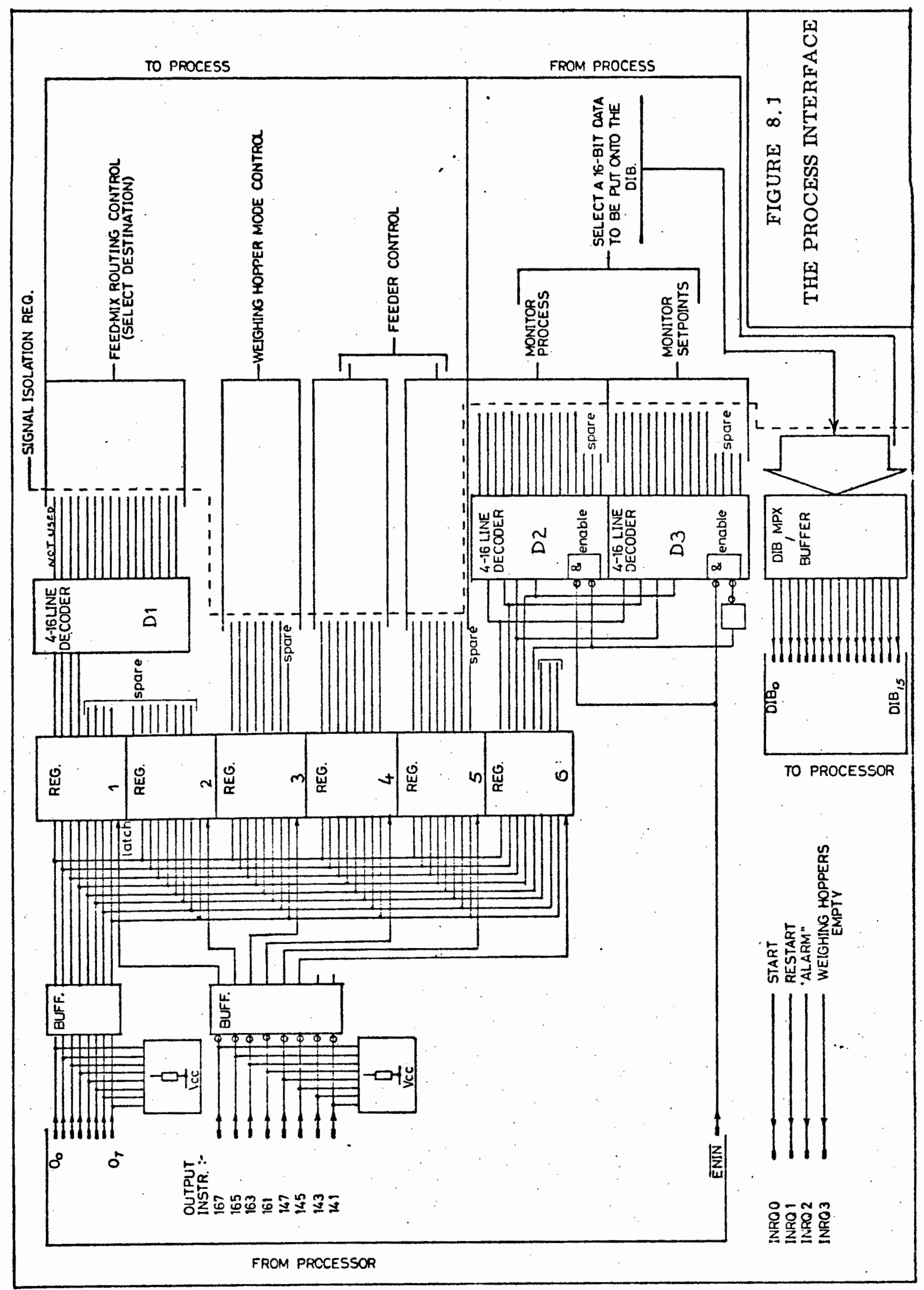


(Chapter $8 . . . .$. continued)

which setpoint (on thumbwheel switches) is to be put on the DIB. The signal ENIN frees the DIB when the processor does not require it and this enables the system's designer to multiplex other (inter-process) signals on to the DIB.

Table 8.1 gives all the software codes which are used to control the process.

\subsection{PROCESS INITIATED COMMNICATION}

One interrupt vector, INRQ2, is reserved for use whenever the process wants to initiate communication with the processor. This will normally be during an emergency. The software routine initiated by $\overline{\text { INRQ2 }}$ would load the A register with $0_{8}$ and output it using instruction $145_{8}$. (See Table 8.2). This would select a sixteen-bit data byte which the system designer could utilize to send the required information to the processor.

The interrupt vector, $\overline{\mathrm{INRQ}}$, is used to inform the processor when all three weighing hoppers are empty.

\subsection{START-UP AND RESTART}

The initial start-up of the processor is via the interrupt vector, INRQO, from the operator's control panel.

Whenever the operator wishes the processor to re-read the setpoints from the thumbwheel switch bank, he can do so via the control panel. This action is commuicated to the processor by INRQI. The processor will cancel all previously determined setpoints and revert to the setpoints currently displayed on the thumbwheel switches. 


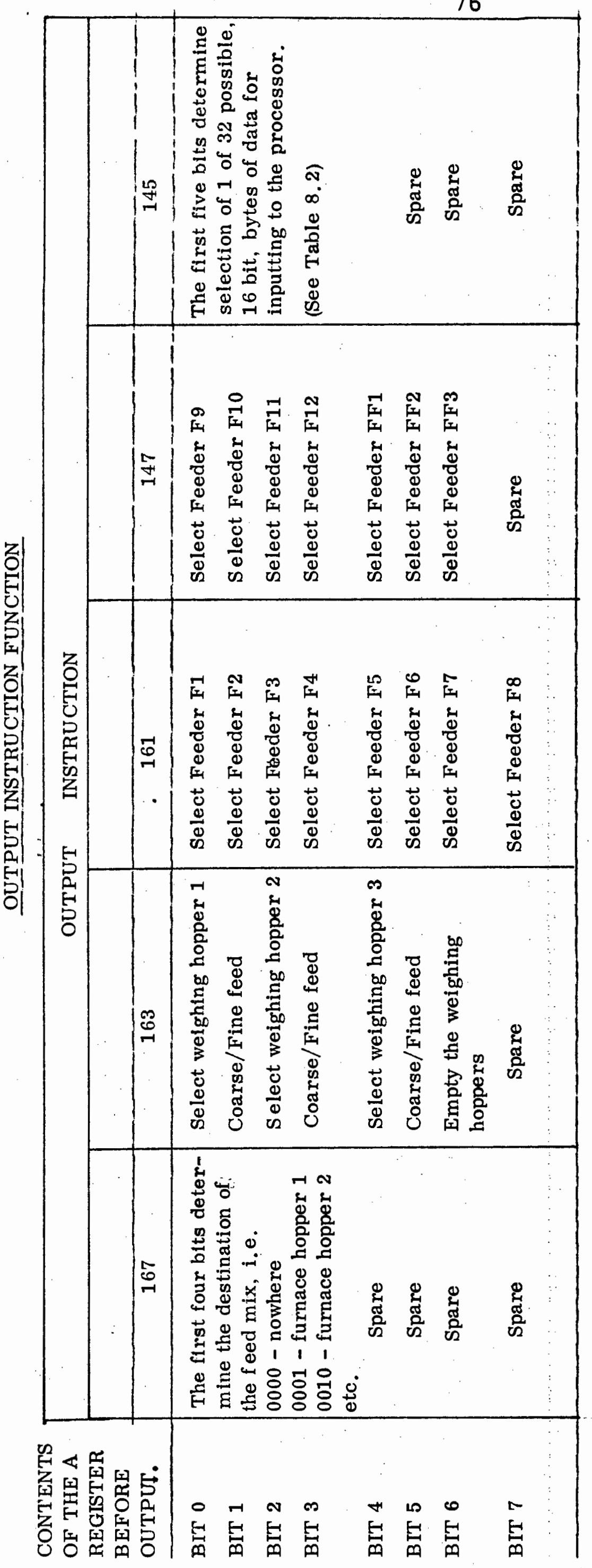


TABLE 8.2

FUNCTION OF THE SELECTED DATA BYTE

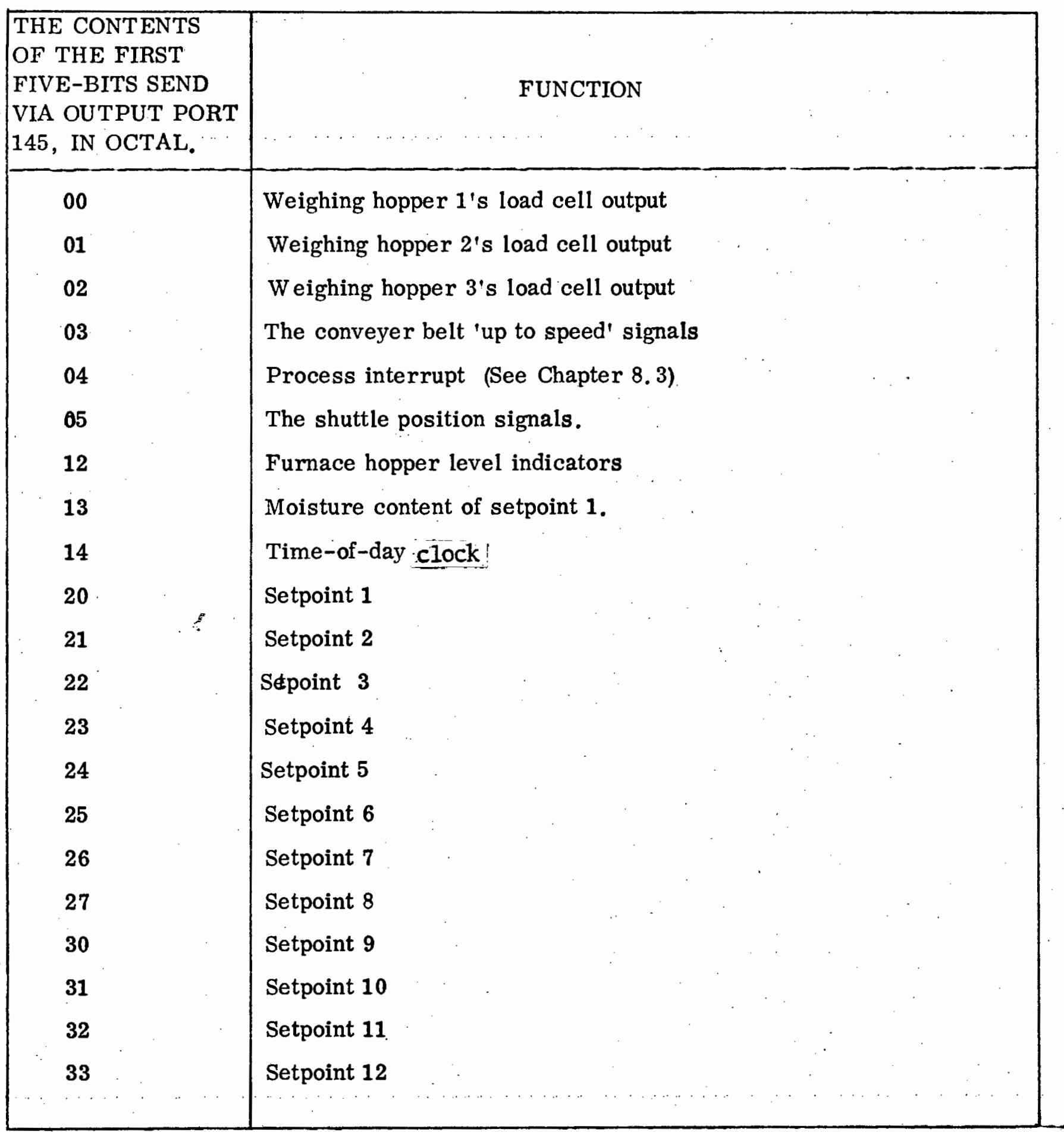




\section{CHAPTER 9}

\section{THE SIMULATOR}

\subsection{INTRODUCTION}

The simulator was designed to ensure an accurate assessment of the processor's capabilities as a control unit for the weighing and transport system of an arc furnace. In particular, it was feared that the microprocessor system would not be fast enough to control the filling of the weighing hoppers.

While the weighing hoppers are being filled, the three load cell outputs have to be continually monitored in order to control the feeders, F1 - F10, correctly. It was therefore important to simulate accurately the time dependant variables of the system.

In order to test the software developed on the prototype, the simulator should display on a mimic panel how the real process would react to the processor's signals.

Attention, however, was concentrated on the need to assess the suitability and capacity of the processor and the software, rather than on simulating all the possible fault conditions which might occur.

As a simulator with a suitable mimic panel would be needed to facilitate industry acceptance and operator training, it was decided not to attempt the simulation on a computer. To ensure easy input/output interfacing between the simulator and the processor, the simulator was designed and built using TTL-logic; therefore analogue simulation was discounted.

\subsection{THE WEIGHING SYSTEM SIMULATOR}

Refer to Figure 9.1 for the block diagram of the system. The three weighing hoppers are simulated identically as is shown for "HOPPER 3".

An up/down counter is used to simulate the effect of the increase or decrease of the load cell voltage. The controller selects the appropriate clock frequency to represent the different feeding rates. 


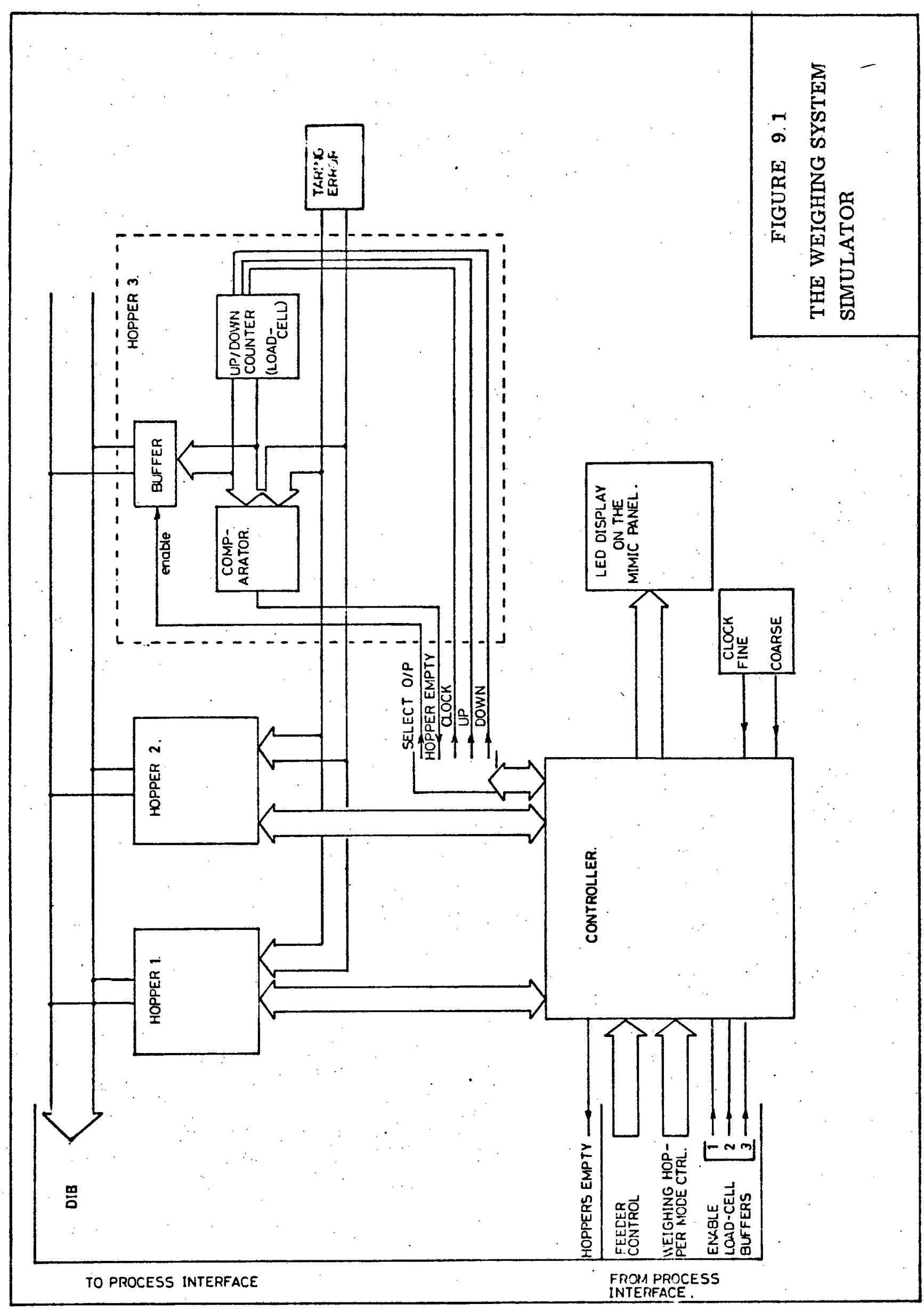


(Chapter 9 - continued)

Flashing light-emitting diode displays on the mimic panel are used to indicate whether any feeder is 'on' or 'off', and also the passage of feed mix onto the conveyor system. Seven-segmented displays are used to indicate the current counter outputs-the quantity of feed mix in any of the weighing hoppers.

Taring errors are simulated by using a bank of thumbwheel switches. When the counter is in the counting down mode (weighing hopper being emptied), the comparator's output indicates when the counter's output equals the taring; error and the controller turns the counter off. (For further details of the weighing system simulator, see "DIGITAL SIMULATION OF A WEIGHING SYSTEM. 1974 B.SC. THESIS No. 21 " by G. Bloch).

\subsection{SIMLATING THE TRANSPORT SYSTEM}

Conveyor belts were simulated using serial shift registers. The clocking frequency of each shift register corresponds to the length of the conveyor belt which it simulates. The different routes and shuttle positions are set up using combinational logic to 'steer' the route to the selected furnace hopper.

Light-emitting diodes on the mimic panel indicate:

(i) which belts are moving

(ii). in which directions the belts are moving

(iii) the presence of feed $\operatorname{mix}$ at the source and destination of each belt.

The present 'state' of the transport system is coded with combinational logic and made available so that the processor can have access to it via the Data Input Bus (DIB). The processor can therefore input signals from the simulator representing:

(i) the belt 'up to speed' signals

(ii) in which directions the belts are moving

(iii) the positions of the shuttles 
(Chapter 9 - continued)

\subsection{FURNACE HOPPER LEVEL INDICATORS}

The furnace hopper level indicators are simulated using toggle switches which are set by the simulator operator. The state of these twoposition toggle switches are read by the processor to determine which furnace hoppers are 'empty'.

\subsection{MOISTURE CONTENT}

A bank of thumbwheel switches represent the percentage (0-99\%) moisture content of the material fed from holding Bin 1. The outputs of the thumbwheel switches can be placed onto the DIB and thus read by the processor. 
SECTION 3 .

SYSTEM SOFTWARE. 
CHAPTER 10

SOFTWARE PHILOSOPHY

The heart and soul of any computer control system is the software the collection of programs which marshalls together the hardware resources and controls their interaction in a predetermined fashion.

\subsection{OBJECTIVES OF SOFTWARE FOR THE PROTOTYPE SYSTEM}

It must be appreciated that the programs written for the prototype are not intended to create fully operational control systems. The programs are to be used in conjunction with a simulator, not a real system. As such, they must perform the following functions:

(i) Evaluate the Hardware:

Are there enough input and output ports and are they of the correct types?

Has sufficient provision been made for intermpt vectors?

Is there provision for enough peripheral devices?

What type of peripherals is suitable?

Is the operator's control panel functional?

Is the mimic panel suitable?

(ii) Evaluate the Programming Aids:

Is it feasible to tackle a programing task of this magnitude in pure machine code?

If not, will an Assembler be sufficient, or should the system's designer have to start thinking in terms of higher-level languages?

Are the hardware programming aids provided suitable for program development?

(iii) Develop Control Strategy:

Computer control systems can be re-programmed: the system designer is not necessarily committed to follow the control strategy used on the prototype. Every effort, however, was made to develop the prototype into a practical control tool. The existing software was written so that additions or alterations can be done vithout disturbing the overall memory structure. 
(Cnapter 10 - continued)

A1l the fault conditions which might occur on a real plant have not been provided for. It is proposed to use the prototype with its simulator in conjunction with users of this type of batch weighing and transport system. This will enable the systems designer to gain knowledge of the type of fault conditions likely to occur and the best corrective measures to be taken. Throughout the software description, suggestions for possible fault detection and correction measures will be given where they are appropriate.

\subsection{SYSTEM FLOW DIAGRAM}

The software consists of a set of sub-routines which are utilized by a main control program. Each sub-routine may make use of other subroutines. All the sub-routines which perform a general function, such as multiplication, $\mathrm{BCD}$ to binary conversions, and so on, are termed utility sub-routines and are not included in the flow diagram description.

The system flow diagram is shown in Figure 10.1. The alphabetically referenced blocks are contained within the main control program. The numerically referenced blocks are implemented by subroutines. The blocks within dotted lines have not been included in the prototype software but would be required in a fully operational system.

(a) The main program is entered after the operator presses a button on the control panel, causing an interrupt to the main program which initiates the following sequence of events:

(1) Further interrupts are disables. (A)

(2) The RAM memory is initialized.

(3) The time of day is recorded on the teletype.

(4) The "general" setpoints are read from thumbwheel switches, stored (2), and recorded by the tele type: (3).

(5) The interrupts are then enabled

(C)

(b) $/ \ldots$ 


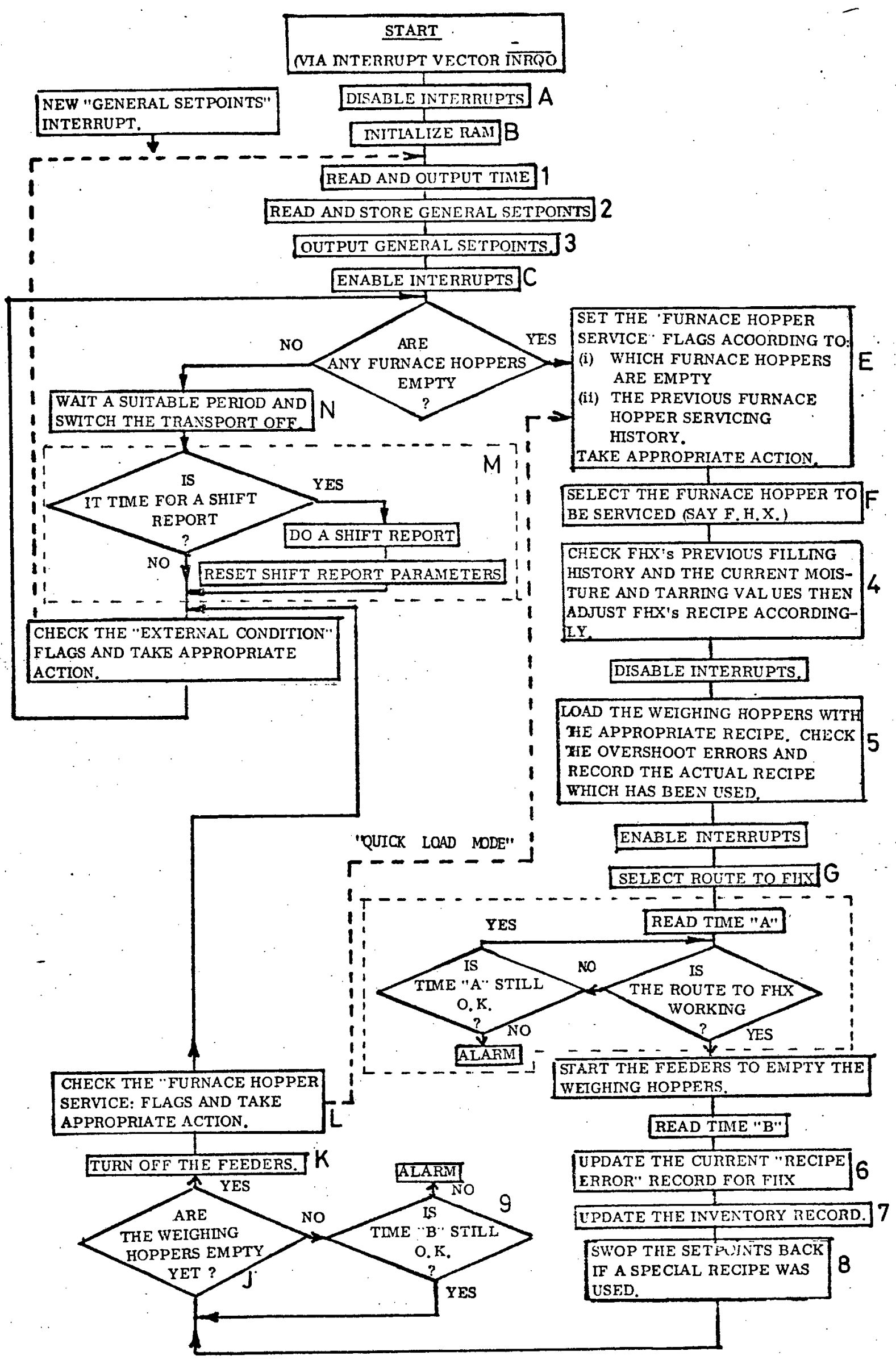

FIGURE 10.1 SYSTEMS FLOW DIAGRAM 
(Chapter 10 - continued)

(b) A check is made to see if any of the furnace hoppers requires servicing. If no furnace hoppers are empty, the following events take place:

(1) The transport system is switched off after enough time has elapsed to ensure that the previous weighing batch has reached its destination $(\mathrm{N})$

(2) A shift report is created if due and the shift report parameters are re-set after the information has been typed out (M)

(3) The "EXTERNAL CONDITIONS" flags are checked, to see if any device (or the process) has requested attention. Typical external conditions checked for are:

(i) The operator's requiring input control parameters to be entered from the teletype.

(ii) The interrupt indicating that a new set of setpoints has been entered on the thumbwheel switches has taken place.

(iii) The central computer requiring attention.

(iv) The process wishing to indicate a certain condition to the processor via the alarm port.

(4) The control is then transferred to (b) above.

(c) If one furnace hopper (or more) is empty the following events take place:

(1) In block $\mathrm{E}$ a check is made to see how many furnace hoppers are empty, and which furnace hoppers are empty. This information, together with the previous furnace hopper servicing history, is used to set flags indicating the present servicing state.

(2) The information processed in (C) (1) above, is used to select which furnace hopper is to be serviced next (say, F.H.(X)).

(3) F.H. (X)'s previous filling history is checked to determine the total recipe errors which were made in the mix previously fed to it. The error, plus the current moisture and taring error, is used to determine the desired feed-mix recipe for F.H.X. 
(Chapter 10- continued)

(4) The interrupts are disabled. The weighing hoppers are filled (5) with the desired recipe. After each feeder is turned off, the overshoot error is determined after a suitable settling-down time. All the overshoot errors are then stored. The intermpts are enabled after the weighing hoppers have been filled.

(5) Next, conveyers and shuttles, which make up the route to F.H. (X) are started (G); a check is made to see when the route is set up and if this is achieved within a certain period.

(6) The feeders which empty the weighing hoppers on to the conveyer system are now started.

(7) While the weighing hoppers are being emptied, the errors in the desired recipe are used to calculate the total recipe error for F.H.(X) and stored (6). Also the inventory record is updated (7).

(8) A check of the "FURNACE HOPPER SERVICE" flags is made to see if a special recipe was used. If so, the old recipe is re-stored (8).

(9) The processor then waits for an interrupt which will indicate that the weighing hoppers are empty $(J)$. It also checks to see that this happens during a specified period.

(10) The feeders which emptied the weighing hoppers are turned off $(\mathrm{K})$.

(11) The "FURNACE HOPPER SERVICE" flags are then interrogated to see if a "fast cycle mode" is required when many (more than six) furnace hoppers were empty $(\mathrm{L})$.

(12) The control is transferred to point (b) (3) above. 
(Chapter 10 - continued)

\subsection{SOFTWARE NOTATION}

In order to streamline the software documentation, it was decided to establish certain conventions.

(a) The following notation is used in the program descriptions:

(1) $A_{R}, B_{R}, C_{R}, D_{R}, E_{R}, H_{R}, L_{R}$ : internal processor registers

(2) LOC. 10-16: memory locations from address $10_{8}$ to address $16_{8}$

(3) C, Z, S, P : internal processor flags, carry zero, sign and parity

(4) $M_{R}$ : current memory location being addressed by $H_{R}$ (HOB) and $L_{R}$

(5) $\mathrm{HOB}:$ highest order byte in a multi-byte reference

(6) LOB : 1owest order byte

(7) hob : highest order bit

(8) Iob : lowest order bit

(9) Unless otherwise indicated, octal numbers are used.

(b) There are 24 different input or output instructions which can be used.

These are listed in Table 6.1. Refer to Table 10.1 for the mnemonics which, for convenience, have been assigned to certain of these input/output instructions.

(c) Detailed program listings can be found in Appendix 6 which is bound separately.

Note: These listings have been produced by a pseudo-dissembler program from the original machine-code.

(d) Memory usage has been assigned as follows:

loc 0-3777 ROM (Read Only Memory)

loc 4000-7777 RAM (Random Access Memory)

loc 10000-17777 ROM (Read Only Memory)

Note:

(i) In order to provide flexibility in the prototype, only random access memory was used. However, the software is structured so that the above memory structure may be adopted. 
TABLE $\quad 10.1$

INPUT/OUTPUT INSTRUCTION MNEMONICS

\begin{tabular}{lll} 
INSTR. & MNENONIC & \multicolumn{1}{c}{ FUNCTION } \\
\hline 101 & ILB & Input lob's of process data \\
103 & IHB & Input hob's of process data \\
105 & PPZ & Input from timer \\
107 & ITY & Input from the teletype \\
123 & OTY & Output to the teletype \\
145 & OSD & Select the next byte of input data \\
147 & OFF & Select feeders F9 to F12 and FF1 to FF3 \\
161 & OSF & Select feeders, F1 to F8 \\
163 & OWH & Weighing hopper control \\
167 & ODC & Select furnace hopper \\
111 & WAR & Read external register \\
117 & IP7 & Input from minicomputer \\
131 & WRA & Load external register \\
133 & ENI & Enable interrupts \\
135 & DSI & Disable interrupts \\
137 & OT7 & Output to minicomputer
\end{tabular}


(Chapter $10^{\circ}$ - continued)

(ii) During program development on the prototype it was found convenient not to use the top kiloword (loc 16000-17777) of available ROM space and to use loc 6600-7777 as ROM memory. This could easily be altered.

(iii) As the program was written in machine code, all references to any of loc 4000-6577 (RAM) should be read as if a variable name is implied. 


\section{CHAPTER 11 \\ THE UTILITY ROUTINES}

In this chapter are discussed the utility routines used by the control program. Such routines are necessary for successful system operation but have no specific relation to the batch-weighing and raw material distribution system.

\subsection{DOUBLE WORD ADDITION}

Sub-rautine ADDM

(a) Purpose

To add the contents of $\mathrm{E}_{\mathrm{R}}$ and $\mathrm{D}_{\mathrm{R}}$ (HOB) to memory locations 4000 and 4001 (HOB).

(b) Limitations

No check is made to see if the maximum negative number possible is exceeded.

(c) Error Checks

If overflow occurs $10 c .4002$ is set to some non-zero number.

(d) Program Location

Address 143-216.

(e) Entrance

Via a sub-routine call to address 143.

(f) Descriptions

The lower order bytes are first added together. The carry bit is added to the higher order bytes when they are added.

11.2 INCREMENT THE MEMORY REGISTERS

Sub-routine INCM

(a) Purpose

To increment the contents of the $\mathrm{H}(\mathrm{HOB})$ and $\mathrm{L}$ registers.

(b) Program Location

Address $220-230$

(c) $/ \ldots$. 
(Chapter 11 - continued)

(c) Entrance

Via a sub-routine call to address 220

11.3 DECREMENT THE MEMORY REGISTERS

Sub-routine DECM

(a) Purpose

To decrement the contents of the $\mathrm{H}(\mathrm{HOB})$ and $\mathrm{L}$ registers.

(b) Program location

Address 231-241

(c) Entrance

Via a sub-routine call to address 231

11.4 OBTAIN 2'S COMPLEMENT

Sub-routine COMP.

(a) Purpose

To obtain the 2's complement of $E_{R}$ and $D_{R}$ (HOB)

(b) Program location

Address 242-260

(c) Entrance

Via a sub-routine call to address 242

11.5 DOUBLE WORD SUBTRACTION

Sub-routine SUBR

(a) Purpose :

To subtract the contents of $E_{R}$ and $D_{R}$ (HOB) from $10 c .4000$ and 4001 (HOB)

(b) Limitations

Similar to 11.1

(c) Error Checks

Similar to 11.1

(d) Program location

Address 261-267

(e) $/ \ldots \ldots$ 


\section{(e) Entrance :}

Via a sub-routine call to address 261.

(f) Other routines used:

(i) Sub-routine ADDM (11.1)

(ii) Sub-routine COMP $(11.4)$

(g) Description : The 2's complekent of $E_{R}$ and $D_{R}$ is obtained and added to $10 \mathrm{c} 4000$ and 4001

\subsection{INTEGER DIVISION}

Sub-routine DIVD

(a) Purpose

To divide $E_{R}$ and $D_{R}$ (HOB) into loc 4003-4006 (HOB). The quotient is placed in loc 4000-4001 (HOB), and the remainder in loc 4003-4006.

(b) Limitations:

The program is written to divide a 2-byte divisor into a 4-byte dividend and the user is required to ensure that these limits are not exceeded.

(c) Error Checks:

See (f) below.

(d) Program Location:

(e) Entrance:

Via a sub-routine call to $10 \mathrm{c} 300$.

(f) Other Routines Used:

(i) Sub-routine COMP $(11.4)$

(g) Description:

The program follows "the pencil-and-paper" method, i.e. it follows the same method as a mathematician would use to do a binary division on paper. 
(Chapter 11 - continued)

\subsection{INTEGER MUTIPLICATION}

sub-routine MULT.

(a) Purpose:

To multiply $E_{R}$ and $D_{R}$ (HOB) by 1 oc $4000-4001$ (HOB). The product is placed in loc 4003-4006 (HOB).

(b) Limitations:

The program is written to multiply 2, 2-byte numbers to obtain a 4-byte product. This sub-routine was written in conjunction with sub-routine DIVD. They are structured so that the intermediate total of a mixed calculation can be 4 bytes ( 32 bits) long.

(c) Error Checks:

None.

(d) Program Location:

Loc 520-706

(e) Entrance:

Via a sub-routine call to $10 \mathrm{c} 520$.

(f) Other Routines Needed:

None

(g) Description:

Similar to $11.6(\mathrm{~g})$.

11.8 NUMBER OUTPUT TO THE TELETYPE

Sub-routine OCHA

(a) Purpose:

To output $E_{R}, D_{R}$ and $C_{R}$ (HOB) to a teletype in the form of a six-digit number plus a sign digit. If $10 \mathrm{c} 4030$ is non-zero the lower order 4 digits only are output.

(b) Limitations:

The number must be within the range $-32,769-327,680$. The user must ensure that $C_{R}=377$ (ail I's) when he wishes to output a negative number. 
(Chapter 11 - continued)

\section{(c) Error Checks}

See (f) below.

(d) Program Location:

1oc. $720-1057$

1oc. $120-141$

(e) Entrance:

Via a sub-routine call to loc. 720 . If the user wants to output a negative number or more than 4 digits, 10c. 4030 must be zero, otherwise loc. 4030 must be non-zero.

(f) Other Routines Used:

(i) Sub-routine COMP (11.4)

(ii) Sub-routine DIVD (11.6)

(iii) Interrupt vector INRQ4 (11.9)

(iv) Sub-routine OUTF (11.9)

(g) Description:

Before being processed the -ve numbers are complemented. The decimal digits are extracted from the binary word by repeated divisions by $10_{10}$. They are then converted to ASCII and stored. If the number was -ve, a -ve sign (in ASCII) is stored with it. These words are then outputted in series.

\subsection{INTERRUPTS WHEN OUTPUTTING TO A TELETYPE} Sub-routine OUTF.

(a) Purpose

Whenever the peripheral controller has completed an output operation to the teletype, an interrupt to $10 \mathrm{c} .40$ is generated. This routine sets a flag in memory to indicate that the teletype is ready to receive data.

(b) $/ \ldots$... 
(Chapter 11 - continued)

(b) Program Location .

Loc. 40-44 (interrupt vector)

Loc. 1060-1103 (set flag)

(c) Entrance

(i) Via interrupt vector INRQ4 or,

(ii) Via a sub-routine call to the 1oc. 1060.

(d) Description

This routine can be entered as the result of an external interrupt; thus all the internal processor register contents must be saved. $A_{R}$ and $B_{R}$ are stored in the external hardware registers so that these registers can be used to save $H_{R}$ and $L_{R}$, the memory address registers. The memory 10c. 4026 is set and all the registers are restored.

11.10 NUMBER INPUT FROM THE TELETYPE

Sub-routine INPN

\section{(a) Purpose}

To input a positive or negative number from the teletype and convert it into a 16-bit binary number.

(b) Limitations

The numbers must be within the range $+32,768$ to $-32,767$.

(c) Error Checks

If the number entered is beyond the above range or if an illegal character is entered, a question mark is printed. If the operator types a "/" character, the number which has been entered is deleted and the required number may be re-entered. The use of INPN must be completed within five minutes; otherwise, the operator must re-negotiate the input transaction.

(d) Program Location 1oc. $1110-1427$

(e) Entrance

Via a sub-routine call to loc. 1110. 
(Chapter 11 - continued)

(f) Other Routines Used

(i) Sub-routine WAIT 2 (porposed for the 5-minute period)

(ii) Sub-routine MLLT (11.7)

(iii) Sub-routine OUTP (11.5)

(iv) Sub-routine COMP (11.4)

(v) Sub-routine IN F (11.11)

(g) Description

The binary word is formed by extracting the decimal digits from the ASCII code words and multiplying by 10. If the first ASCII code is a -ve sign, the 2's complement of the binary word is taken.

\subsection{INTERRUPTS WHEN INPUTTING FROM THE TELETYPE}

Sub-routine INF

(a) Purpose

Whenever the peripheral controller has completed an imput operation using the teletype, an interrupt to 1oc. 50 is generated. This routine sets a flag in memory to indicate that a character has been entered.

(b) Program Location

$$
\begin{aligned}
& \text { 1oc. } 50-54 \text { (interrupt vector) } \\
& \text { 1oc. } 1430-1461 \text { (set flag) }
\end{aligned}
$$

(c) Entrance

(i) Via interrupt vector INRQ5, or

(ii) A sub-routine call to $1 \propto 0.1430$

(d) Description

See Chapter 11.9 (d) 
(Chapter 11 - continued)

\subsection{BCD TO BINARY CONVERSION}

Sub-routine BCDTB

\section{(a) Purpose}

To convert the $B C D$ coded contents of the $C_{R}$ and $B_{R}$ (HOB) into binary information. The binary number is placed in 1oc. 4000 and 4001 (HOB).

(b) Limitation

The highest order $\mathrm{BCD}$ bit (the sign bit) is ignored and a positive number is always produced.

(c) Program Location 10c. $2040-2106$

(d) Entrance

Via a sub-routine call to $10 \mathrm{c} .2040$

(e) Other Routines used

(i) Sub-routine CALC $(11.13)$

(f) Description

The $\mathrm{BCD}$ digits (groups of four bits) are separated, multiplied by the corresponding order of $10_{10}$ and added together.

\subsection{SUB-ROUTINE CALC.}

(a) Purpose

To be used with sub-routine BCDTB

(b) Program location Loc. $2110-2150$

(c) Entrance

Via a sub-routine call to 1oc. 2110

\subsection{BINARY TO BCD CONVERSION}

Aub-routine BTBCD

(a) Purpose

To convert the binary number in 1oc. 4003 and 1oc. 4004 (HOB) into four $B C D$ numbers. The four-bit binary (BCD) numbers are placed in loc. 4045 and 4046 with the units bit-string occupying the 4 lower-order bit 
(Chapter 11 - continued)

positions of 1oc. 4045 .

(b) Limitations

The binary number may not be -ve.

(c) Program location

1oc. 2155-2221

(d) Entrance

Via a sub-routine call to loc. 2155

(e) Description

The $\mathrm{BCD}$ digits are extracted by repeatedly dividing by 10 and using the resulting remainders.

11.15 TO OUTPUT ALPHA NUMERIC DATA VIA THE TELETYPE

Sub-routine OUTP.

(a) Purpose

To print the contents (ASCII characters) of all memory loc. between two specified addresses. The first address is specified by $H_{R}$ (HOB) and $C_{R}$. The last address is specified by $H_{R}(H O B)$ and $B_{R}$.

(b) Limitations

The sub-routine can only output a string of memory locations of which the higher order byte $\left(\mathrm{H}_{\mathrm{R}}\right)$ is the same.

(c) Program Location

1oc. $2011-2037$

(d) Entrance

Via a sub-routine call to loc. 2011.

11.16 CONTROL INPUT VIA THE TELETYPE Sub-routine CINP

(a) Purpose

To examine a character received from the teletype and to branch to the routine which executes the required action as indicated by that character. (b) $/ \ldots$. 
(Chapter 11 - continued)

(b) Limitations

Due to periods during which the intermpts may have been disabled, the operator has to wait for a response from the processor after he has depressed a key indicating that a specific control action is required. CINP caters for a maximm of 5 different input control characters.

(c) Error Checks

When any character is entered which "CINP" does not recognise or expect, the following is printed : "?", "RETURN", "LINE FEED"

(d) Program Location

1oc. 10477-10562

1oc. $3540-3547$

(e) Entrance

via a sub-routine call to $10 \mathrm{c} .10477$

(f) Other Routines Used

(i) Sub-routine OUTP (11.15)

NOTE: "CINP" jumps to one of several different routines according to which control action is desired as specified by the input-fed character.

(g) Description

When the main program examines the "EXTERNAL CONDITION" flags and determines that the operator has entered a character from the teletype, a call to CINP is made. The character is examined and a branch is made to the appropriate routine.

11.17 RECORD TIME OF DAY

Sub-routine TIME 1 .

(a) Purpose

To read the time from a digital clock (in BCD code) and store it in 1oc. 4045 (minuites and tens of minutes) and Loc. 4046 (hours and tens/... 
(Chapter 11 - continued)

tens of hours )

(b) Program Location

Ioc. $11730-11775$

(c) Entrance

Via a sub-routine call to 1oc. 11730

11.18 PRINT TIME OF DAY

Sub-routine TIMP

(a) Purpose

To convert the $\mathrm{BCD}$ coded contents of 1oc. 4045 and 4046 into ASCII coded characters and to print them onto the teletype. (See Chapter 11.17)

(b) Program Location Loc. $12000-12134$

(c) Entrance

Via a sub-routine call to loc. 12000

(d) Other Routines Used

(i) Sub-routine OUTP (11.15)

11.19 TO TIME A SHORT DELAY

Sub-routine TIMD

(a) Purpose

To enable a program to estimate any period from 1 to 127 seconds without having to use "marking-time" routines. The period to be timed corresponds to the contents of $D_{R}$.

(b) Limitations

Timing to within 1 second is only possible if TIMD is used at least once every second.

(c) Program Location 1oc: $13300-13340$

(d) Entrance Via a sub-routine call to 1oc. 13300. 


\section{(e) Description}

Input instruction code 105 causes the output of an eight-bit counter rumning at one hertz to be read. When the progranmer wants to program a short time delay into a routine, he is required to read the timer and store the count in a specific memory location. When the programmer wishes to use TIMD, $M_{R}$ must point to the memory location, where the count is stored and $D_{R}$ must contain the desired timing period. TIMD sets the carry flip-flop as follows:
$\mathrm{C}=0$ : when the timed period is not over
$C=1$ : when the timed period is over 
CHAPTER 12

THE CONTROL PROGRAMS

\subsection{LOADING THE WEIGHING HOPPERS}

Sub-routine CONW, Sub-routine WEIGH

These two sub-routines together control the proportioning of the ingredients which are fed into the weighing hoppers. The inputs to these routines are:

(i) The desired setpoints which will have been converted into the $B C D$ code and stored in specific memory locations, and

(ii) The $\mathrm{BCD}$ coded outputs of the three load cells which are located underneath the weighing hoppers.

These routines control the feeders which feed contents of the holding bins into the weighing hoppers.

Sub-routine CONW is the main controlling program and sub-routine WEIGH is its main sub-routine.

\subsubsection{SUB-ROUTINE WEIGH}

(a) Purpose

Every time this sub-routine is entered, one of the following actions can take place. The specific action is controlled by "flags" in memory which are continually interrogated and updated by WEIGH and CONW.

(i) Return to CONW if the selected weighing hopper has been loaded.

(ii) Start the selected feeder.

(iii) Stop the selected feeder if its setpoint has been reached.

(iv) Switch the selected feeder from the coarse feed to the fine feed mode if the weight is within $90 \%$ of the setpoint.

(v) Return to CNW if not enough time has el apsed for the selected load cell output to stabilize after a feeder has been stopped.

(vi) Record the actual setpoint used (including overshoot error) after the selected load cell' $\hat{s}^{\prime}$ output has settled down. 
(Chapter 12 - continued)

(vii) If the current feeder has completed its task, then select the next feeder and its setpoint.

(b) Limitations

WEIGH is controlled from CONW; CONW decides which feeder (and weighing hopper) is to be serviced. As WEIGH can only "look" at the one load cell output at a time but material may be feeding into all three weighing hoppers, there will be a certain feeding rate (mass per unit time) beyond which the feeders may not be turned off quickly enough, resulting in excessive overshoot errors.

(c) Program Location

$$
\begin{aligned}
& \text { 1oc. } 2260-2574 \\
& \text { 1oc. } 2705-2767 \\
& \text { 1oc. } 3000-3010
\end{aligned}
$$

(d) : Entrance

Via a sub-routine call to $10 \mathrm{c} .2260$.

(e) Other Routines Used.

(i) Sub-routines CUFE (12.1.3)

(ii) Sub-routine FLAG (12.1.4.)

(iii) Sub-routine NESP (12.1.5.)

(iv) Sub-routine FEED $(12.1 .6$.

(v) Sub-routine TIMD $(11.1 .9$.

(vi) Sub-routine COMS $(12.1 .7$.

(f) Description

Refer to the flow chart, Figure 12.1.

\subsubsection{SUB-ROUTINE CONW}

\section{(a) Purpose}

To perform the function of a main controlling routine for the loading of the weighing hoppers. CONW controls the initiating, sequencing, ending, and error checks for the loading process. 


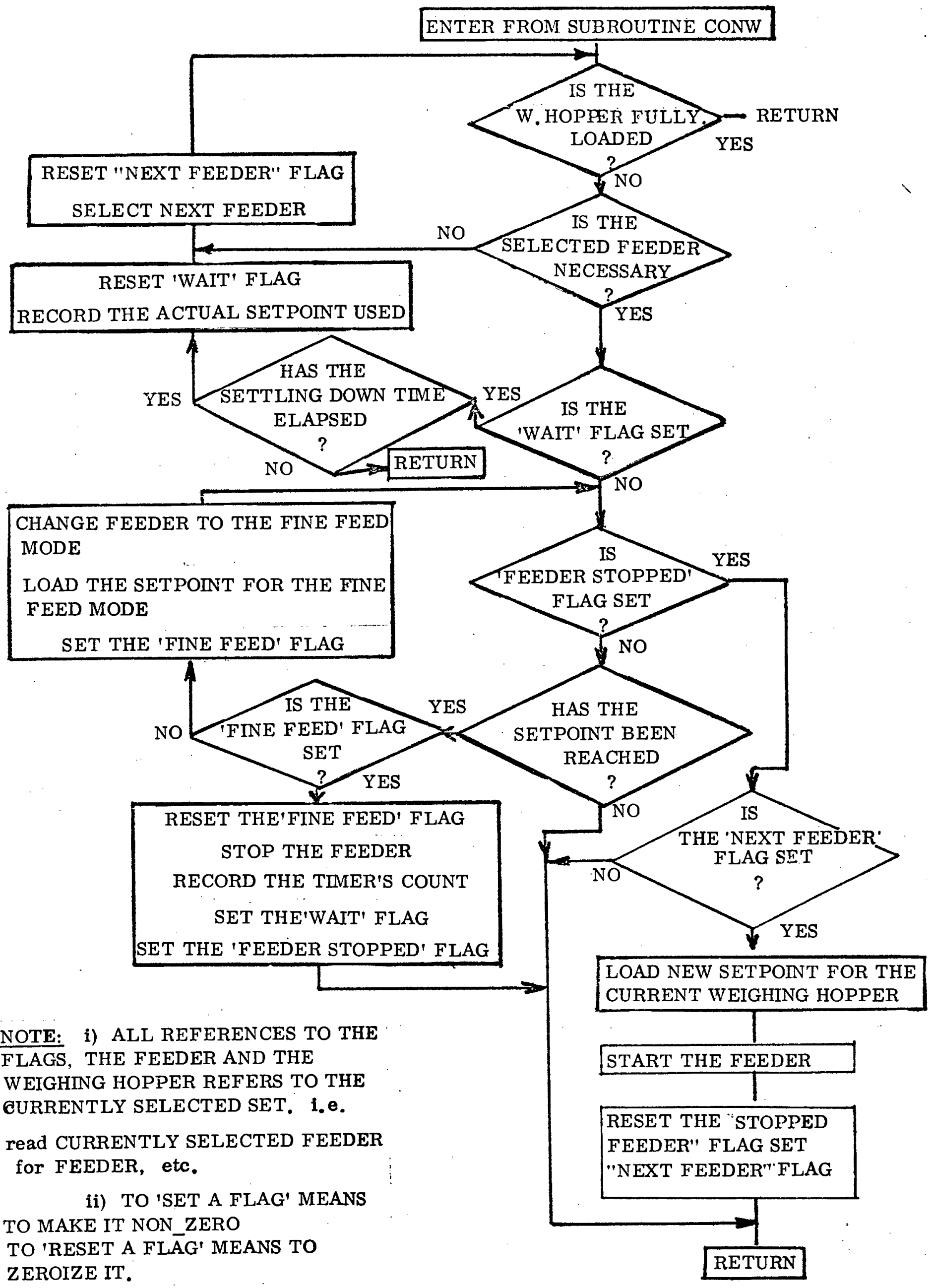

FIGURE 12.1 LOADING THE WEIGHING HOPPERS. 


\section{(b) Limitations}

See sub-routine WEIGH

(c) Error Checks

Recommended error checks are that a check should be made that each feeder performs its function and that the weighing hoppers are loaded within a specified maximm period.

(d) Program Location

loc. $13420-13543$

(e) Entrance

Via a sub-routine call to loc. 13420 .

(f) Other Routines Used

(i) Sub-routine COSP $(12.1 .8)$

(ii) Sub-routine WEIGH (12.1.1)

(iii) Sub-routine ERR (Proposed)

(g) Description

Refer to flow chart, figure 12.2 .

ERR is a sub-routine whose function will be to perform the error checks recommended in (c) above.

12.1.3 TO HAVE ACCESS TO THE CURRENTLY SELECTED FEEDER

Sub-routine CUFE

(a) Purpose

CUFE places the pointer (1oc. 4771) to the currently selected weighing hopper in $C_{R}$. It also places the pointer (loc. 4730,4733 , or 4736 ) selecting the current feeder in $B_{R}$. Upon exit from CUFE, $M_{R}$ contains this memory location.

(b) Program Location

Loc. $2600-2614$.

(c) Entrance

Via a sub-routine call to loc. 2600. 


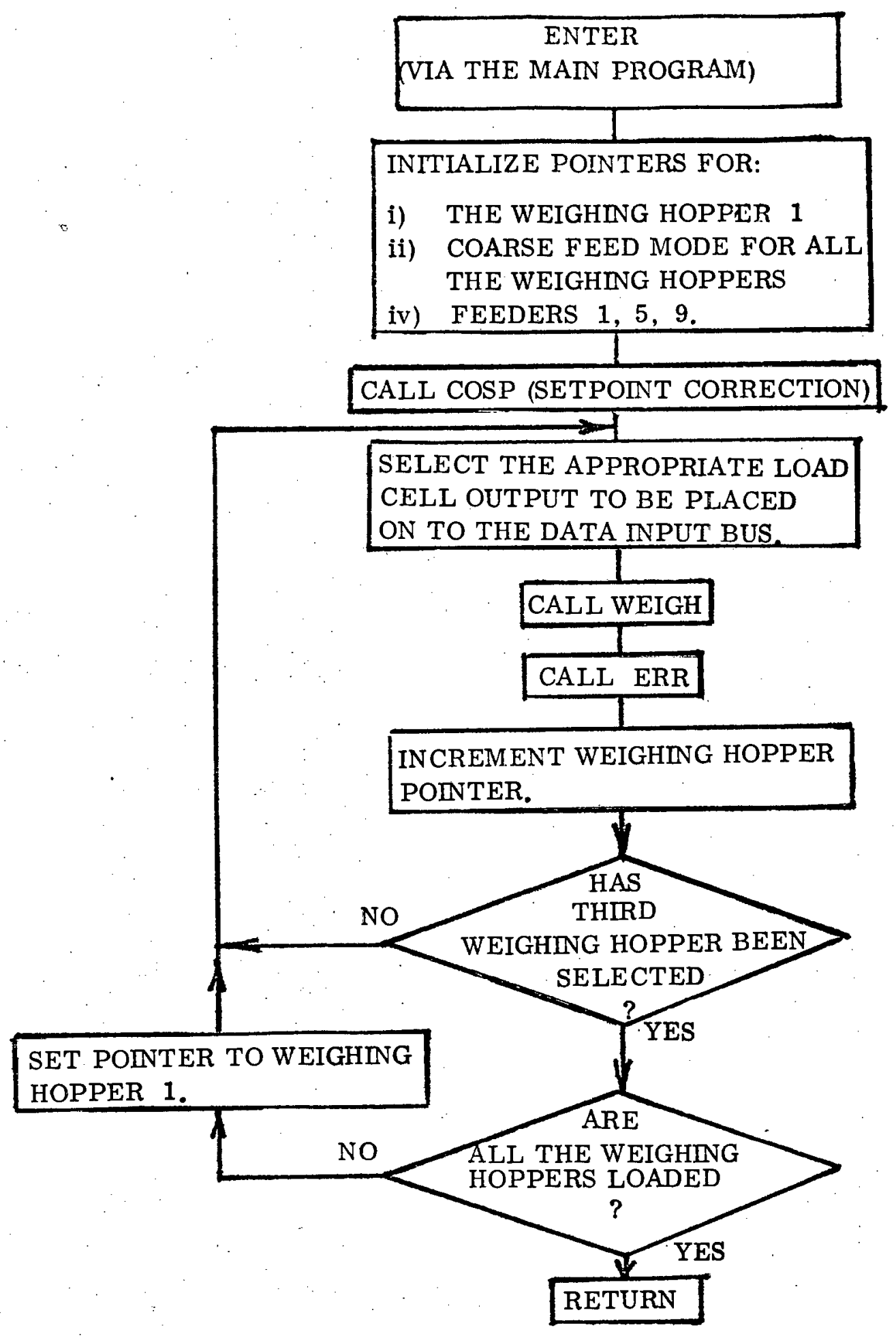

FIGURE 12.2 TO CONTROL THE WEIGHING HOPPER LOADING 
(Chapter 12 - continued)

\subsubsection{TO HAVE ACCESS TO THE CURRENTLY RELEVANT FLAGS}

Sub-routine FLAG.

(a) Purpose

To make $M_{R}$ point to the relevant address in memory which contains the desired program flag and is used by WEIGH.

(b) Program Location

Loc. $2615-2630$.

(c) Entrance.

Via a sub-routine cill to $10 \mathrm{c} .2613$.

(d) Description

There are four flags relevant to each weighing hopper: NEXT FEEDER; WAIT, STOPPED and FINE FEED. These are numbered 0, 1, 2 and 3, respectively. This number must be loaded into $A_{R}$ before entrance to FLAG. Upon exit from FLAG, $M_{R}$ will point to the memory location containing information relevant to the flag.

\subsubsection{TO LOAD THE NEXT SETPOINT}

Sub-routine NESP

(a) Purpose

After the previous setpoint has been reached, NESP is used to select the next appropriate setpoint for a particular. weighing hopper.

(b) Program Location

Loc. $2635-2702$.

(c) Entrance

Via a sub-routine call to loc. 2635 .

(d) Other Routines Used

Sub-routine CUFE $(12.1 .3)$

(e) Description

For each weighing hopper there are specific memory locations which store the current setpoint for a particular feeder to that hopper. NESP loads these locations as required. 
(Chapter 12 - continued)

12.1.6 TO CONTROL THE FEEDERS WHICH LOAD THE WEIGHING HOPPERS

Sub-routine FEED

(a) Purpose

To turn the currently selected feeder on or off.

(b) Program Location

(c) Entrance. 2730-2767

Loc. $13150-13275$.

Loc. 2730-2767

Via a sub-routine call to $10 \mathrm{c} \cdot 13150$.

(d) Description

If $E_{R}$ is non-zero the currently selected feeder is turned on.

If $E_{R}$ is zero the currently selected feeder is turned off.

12.1.7 TO CHECK IF THE CURRENTLY SELECTED SETPOINT HAS BEEN REACHED Sub-routine COMS

(a) Purpose

To read the relevant load cell output to see if the current setpoint has been reached.

(b) Program Selection

Loc. $1770-2010$

(c) Entrance

Via a sub-routine call to $10 \mathrm{c} .1770$

(d) Description

COMS sets the carry-flag if the setpoint has been reached.

12.2 TO PERFORM ERROR CORRECTIONS ON THE CURRENTLY SELECTED SETPOINTS Sub-routine COSP

(a) Purpose

COSP does the following manipulations on the setpoints of the currently selected furnace hopper:

(i) Reads/..... 
(Chapter 12 - continued)

(i) Reads the percentage moisture content of the ingredients in holding bin one and adds the required amount to setpoints $1,2,3$ and 4 , (remembering that they are accumulative).

(ii) Reads the load cell outputs when the weighing hoppers are supposedly empty and adjusts the setpoints according to these taring errors. All the setpoints corresponding to each of the weighing hoppers are adjusted for the taring error.

(iii) Corrects the setpoints according to the overshoot errors from the previous occasion when the currently selected furnace hopper was serviced.

(iv) Calculates $90^{\circ}$ of the setpoints as determined by i), ii) and iii) above.

(v) Converts the corrected setpoints and their $90 \%$ equivalents into the $B C D$ code and stores these ready for use by the weighing hopper loading routines.

\section{(b) Limitations}

Moisture correction is only done to the first order of accuracy. For example, if the recipe requires $100 \mathrm{~kg}$ of the ingredient which contains, say, $10 \%$ moisture, then COSF will correct setpoint according to the formulae:

$$
\begin{aligned}
\text { Corrected setpoint } & =100+\frac{100}{1} \times \frac{10}{100} \\
& =110 \mathrm{~kg}
\end{aligned}
$$

Remember that the $10 \%$ of the ingredient added also contains $10^{\circ}$ or 1 KG of misture, etc. In this case, therefore, too little will have been added to the setpoint.

(c) Error Checks

Provision has been made for an alarm indication if a taring error of more than $256 \mathrm{~kg}$ is detected.

(d) Program Location

Loc. $3020-3533$ 
(Chapter 12 - continued)

(e) Entrance

Via a sub-routine call to $10 c .3020$.

(f) Other Routines Used
i) Sub-routine $\mathrm{BCDTB}(11.12)$
ii) Sub-routine FISP $(12.3)$
iii) Sub-routine MLT (11.7)
iv) Sub-routine DIVD $(11.6)$
v) Sub-routine $\operatorname{COMP}(11.4)$
vi) Sub-routine $\mathrm{ADDM}(11.1)$
vii) Sub-routine BTBCD (11.14)

(g) Description

Refer to the flow chart, Figure 12.3.

12.3 TO ADDRESS THE CURRENT SETPOINT

Sub-routine FISP

(a) Purpose

FISP sets $M_{R}$ to the memory location address in which the first byte of the desired setpoint is stored.

(b) Progress Location

Loc. $12614-12701$.

(c) Entrance

Via a sub-routine call to 1oc. 12614.

(d) Other Routines Used.

i) Sub-routine MUT (11.7)

ii) Sub-routine ADDM (11.1)

(e) Description

Throughout the control porcess a pointer selecting which furnace hopper is to be serviced is stored in 1oc. 4063. Similarly, loc. 4064 is/.... 
SUBROUTINE COSP

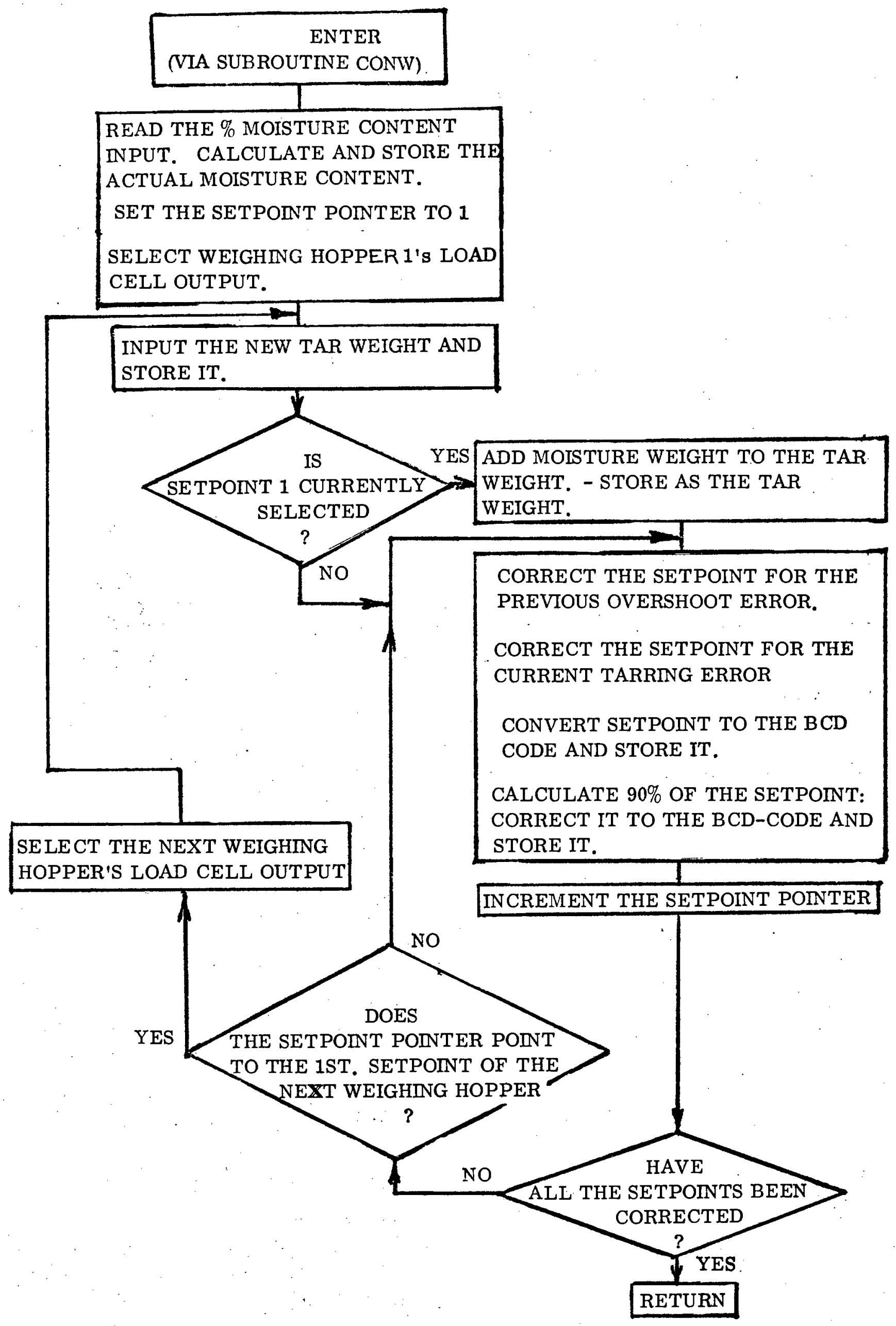

FIGURE 12.3 TO PERFORM ERROR CORRECTIONS ON THE CURRENTLY SELECTED SETPOINTS. 
(Chapter 12 - continued)

is used for a setpoint pointer. FISP makes use of these two pointers to set up $M_{R}\left(H_{R} \& I_{R}\right)$. The overshoot error for each setpoint is: stored ${ }_{\Lambda}$ exactly . 1000 memory locations away from the setpoint; and the programmer can therefore increment $H_{R}$ twice in order to set up $M_{R}$ to the memory location address containing the first byte of the overshoot error.

\subsection{TO DETERMINE THE GENERAL SETPOINTS}

Sub-routine RESP.

(a) Purpose

To read the thumbwheel switch outputs which specify the "general". setpoints for each furnace hopper, convert these values into 2-byte binary numbers and store them as the current setpoints.

(b) Error Checks

If any succeeding setpoint is smaller than the preceding setpoint and both these setpoints apply to feeders feeding into the same weighing hopper, then that setpoint is made equal to the preceding one. Because the setpoints are accumulative, this will imply that nothing will be fed from the corresponding holding bin. Therefore, if the plant hardware or the desired recipe dictates that a certain holding bin should not be used, a zero indication on the corresponding setpoint will be the only change necessary.

(c) Program Location Loc. $12135-12332$.

(d) Entrance

Via a sub-routine call to loc. 12135.

(e) Other Routines Used

i) Sub-routine BCDTB (11.12)

ii) Sub-routine FISP (12.3)

(f) Description/...... 


\section{(f) Description}

Refer to Flow Chart, Figure 12.4 .

\subsection{TO PRINT THE SETPOINTS ONTO THE TELETYPE}

Sub-routine ROSP : Sub-routine ROGP.

Together these two sub-routines list the setpoints. ROSP is used during a shift report to list the setpoints which currently apply to each furnace hopper (see Figure 12.5). ROGP is used to list the general setpoints which apply to all the furnace hoppers after the system has just been started up or re-started upon the operator's request. (See Figure 12.6)

\subsubsection{SUB-ROUTINE ROSP}

(a) Purpose

As discussed above.

(b) Program Location

$$
\begin{aligned}
& \text { Loc. } 12340-12520 \\
& \text { Loc. } 12710-12760 \quad\} \quad \text { The output literal } \\
& \text { Loc. } 13000-13125 \quad \text { lists coded in ASCII }
\end{aligned}
$$

(c) Entrance

Via a sub-routine call to loc. 12340.

(d) Other Routines Used
i) Sub-routine OUTP $(11.15)$
ii) Sub-routine OCHA (11.8)
iii) Sub-routine FISP (12.3)

12.5.2. SUB-ROUTINE ROGP

\section{(a) purpose}

As discussed above.

(b) Program Location

$$
\begin{aligned}
& \text { Loc. } 14410-1431 \\
& \text { Loc. } 1432-1467 \text { The output literal list coded in ASCII } \\
&
\end{aligned}
$$




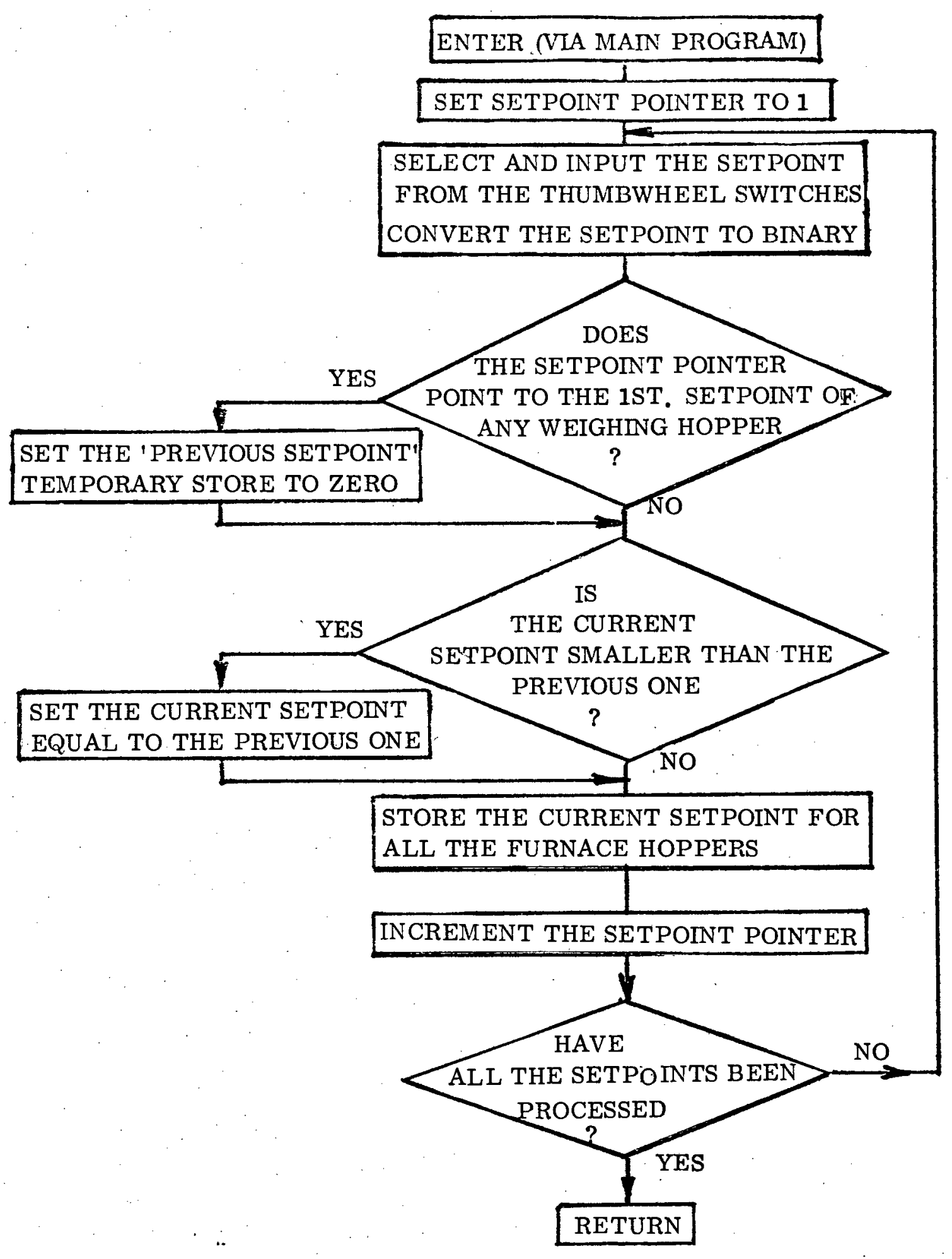

FIGURE 12.4 TO DETERMINE THE GENERAL SETPOINTS 
THE CURFENT SETPOINTS ARE:

\section{SETPRINTS:}

\begin{tabular}{|c|c|c|c|c|c|c|c|c|c|c|c|c|}
\hline H. & 1 & 2 & 3 & 4 & 5 & 6 & 7 & 8 & 9 & 10 & 11 & 2 \\
\hline & 0110 & 275 & 0350 & 0350 & 0100 & 0150 & 0150 & 0150 & 250 & & & \\
\hline 02 & 110 & 5 & & 0 & 0 & & 0 & 50 & 0 & n & r & \\
\hline 003 & 0110 & 0275 & 035 & 50 & 100 & 0 & 0 & 50 & 50 & co & 10 & \\
\hline 004 & 0110 & 0275 & 0350 & 0350 & 0100 & 0150 & 0150 & 0150 & 0250 & O70n & 1000 & 1 \\
\hline 0005 & 011 & 0275 & 0350 & 0350 & 0100 & 0150 & 0150 & 0150 & 0250 & 0700 & 1000 & 05 \\
\hline 006 & 0120 & 0300 & 0375 & 0405 & 0100 & 01 & 01 & in & 0250 & 0700 & 1000 & 1050 \\
\hline Jur & 0120 & 0 & 0375 & 0405 & 0100 & 0150 & 0150 & 0150 & 0250 & 0700 & 1000 & 1050 \\
\hline 0008 & 0 & 0300 & 0375 & 0405 & 0100 & 0 & 0150 & 0. & 0250 & 0700 & 1000 & 1050 \\
\hline 009 & 0110 & 0275 & 0350 & 0350 & 0100 & 0 & 0 & 0 & 0250 & 0700 & $10 \cap 0$ & 1050 \\
\hline 10 & 0110 & 0275 & 0350 & 0350 & 0100 & 0150 & 0150 & 50 & 0250 & 0700 & 0 & 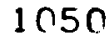 \\
\hline 011 & 0110 & 0275 & 0350 & 0350 & 0100 & 0150 & 0150 & 0150 & 0250 & 0700 & 1000 & 1050 \\
\hline 12 & 10 & 275 & 0350 & 0350 & 0100 & 0150 & 0150 & 0150 & 02.50 & 0700 & $1 \cap 00$ & 1050 \\
\hline & 0 & 0275 & 0 & 0 & 01 & 0150 & 0150 & 0 & 0250 & 0700 & 1000 & 1050 \\
\hline & & 0275 & 0350 & 0350 & 01 & 0150 & 50 & 0. & 0250 & 0700 & 1000 & 1050 \\
\hline 11 & 110 & 275 & 0350 & 0350 & 0100 & 150 & 0150 & 0150 & $025 n$ & 0700 & 1000 & 15 \\
\hline
\end{tabular}

FIGURE 12.5 A SETPOINT REPORT

TIME: 1100 HOUFS

THE GENERAL SETFOINTS ARE:

SETFOINTS:

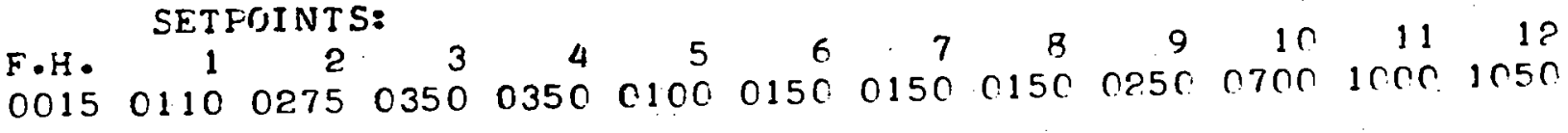

FIGURE 12.6 A GENERAL SETPOINT REPORT 
(Chapter 12 - continued)

(c) Entrance

Via a sub-routine call to 1oc. 14410 .

(d) Other Routines Used

Sub-routine OUTP (11.15)

Note: ROGP does not exist in the normal manner but performs a jump into ROSP (Chapter 12.4.1).

(e) Description

ROGP prints the "general setpoints" heading. It then sets pointers in memory to indicate that only the last furnace hopper's setpoints are still to be outputted. ROSP is then entered at loc. 12357 so that only the last furnace hopper's setpoints are printed as normal: Note that it is only intended to use ROGP immediately after RESP (Chapter 12.4) when the setpoints for all the furnace hoppers will be equal.

\subsection{TO CHANGE THE CONTROL PARAMETERS FROM THE TELETYPE}

Routine PERC. Routine LOCO.

These routines are used to make adjustments to the setpoints from the teletype. They are entered via jump instructions from sub-routine CINP (11.16). They are terminated by a "return" and will therefore return directly to the calling program. Refer to the flow chart, figure 12.9. 12.6.1. ROUTINE LOCO

\section{(a) Purpose}

To enable the operator to effect changes on the recipe of the feed mix to be fed to certain specified furnace hoppers. The operator can specify for how many batches per furnace hopper the altered recipe is to be used before reverting to the 'old' recipe. An example of the printout produced during such a communication between the operator and processor is shown in Figure 12.7. Note that the underlined printing is 


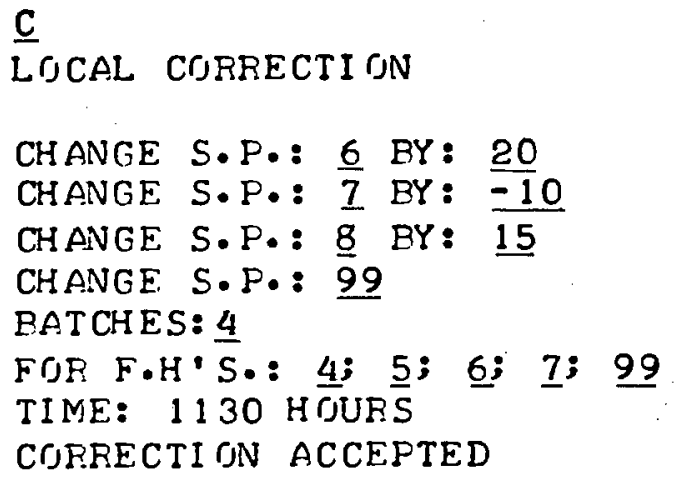

$\underline{\mathrm{C}}$

LOCAL CORRECTIINN

CHANGE S.P.: 6 BY: 20

CHANGE S.P.: 7 BY: -10

CHANGE S.P.: 8 BY: 15

CHANGE S.P.: 99

BATCHES: $\underline{4}$

FOR F.H'S.: 4; $\underline{5} ; \underline{6} ; \underline{7} ; \underline{99}$

TIME: 1130 HOURS

CORRECTION ACCEPTED

FIGURE 12.7 TEMPORARY ADJUSTMENT TO AFFECT A LOCAL CORRECTION

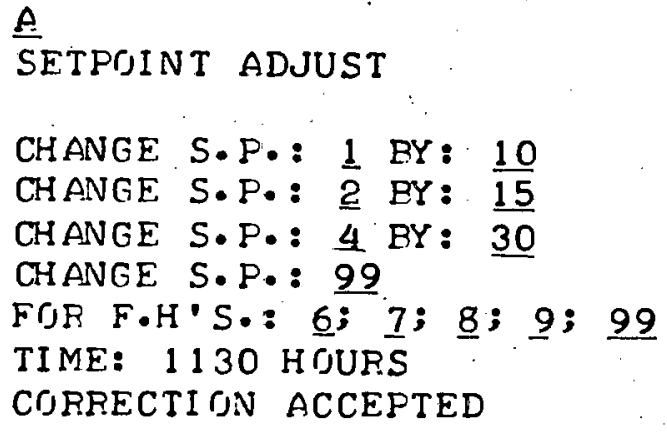

FIGURE 12.8 A PERMANENT SETPOINT ADJUSTMENT 


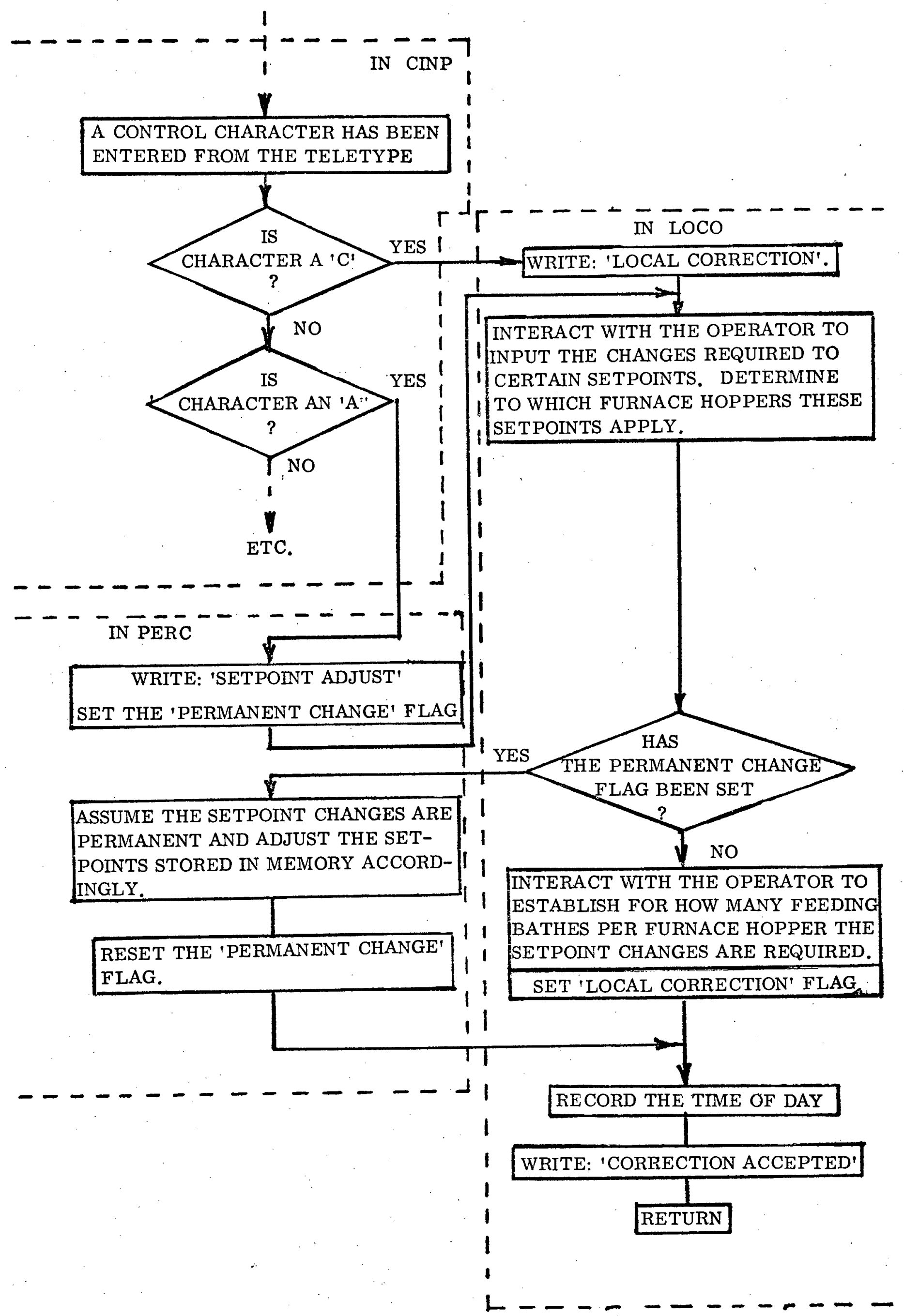


(Chapter 12 - continued)

done by the operator from the teletype keyboard.

(b) Limitations

A maximum of 256 batches per furnace hopper can be specified.

(c) Error Checks

See Section (f) below.

(d) Program Location

Loc. $14647-15250$

Loc. $6600-6750$ : the literal list for output coded in ASCII

(e) Entrance

Via a jump to 1oc. 14647 .

(f) Other Routines Used

(i) Sub-routine OUTP $(11.15)$

(ii) Sub-routine TIREC (proposed)

(iii) Sub-routine INPN (11.10)

(iv) Sub-routine SPAD (12.6.3)

(v) Sub-routine Time 1 (11.17)

(vi) Sub-routine $(11.18)$

(g) Description

Refer to the flow chart, figure 12.9.

12.6.2 SUB-ROUTINE PERC.

(a) Purpose

To enable the operator to effect changes in the recipe to the feed mix

to be supplied to specified furnace hoppers. These changes will be used

for all the succeeding batches. (See Figure 12.8).

(b) Program Location

Loc. $14470-114646$

Loc. $14400-14404$

Loc. $6600-6750$ : the literal list of characters for output coded in ASCII

(c) Program $/ . .$. . 
(Chapter 12 - continued)

(c) Program Entrance

(i) Via a jump to loc. 14625

(ii) The program is re-entered from Loc0 via a jump to loc. 14470 .

(d) Description

Refer to the flow chart, figure 12.9.

12.6.3. TO ADUUST THE SETPOINTS ACCORDING TO OPERATOR SPECIFICATIONS Sub-routine SPAD

(a) Purpose

During a setpoint adjustment the operator specifies the change in the setpoint required according to this change.

(b) Program location

$$
\text { 10c. } 1620-1710
$$

(c) Entrance

Via a sub-routine call to loc. 1620 .

12.7 TO PRODUCE A SHIFT REPORT

Sub-routine INVEN

(a) Purpose

To print onto the teletype an inventory of:

(i) The raw materials used from each holding bin, (10c. 5650 - 5677);

(ii) The feed mix fed to each furnace hopper (1oc. 5014 - 5051);

(iii) The total mass of raw material used (1oc. 5060 - 5062).

INVEN also lists the current setpoints required for each furnace hopper.

See Figure 12.10 for an example of such a shift report.

(b) Program Location

Ioc. $3540-3766$

1oc. $10570-.10746$

1oc. $6747-7127$ : literal list of the output characters coded in ASCII.

(c) Entrance/..... 
INUENTOFY REFORT

TIME: OB CO HOURS

THE CUARENT SETPOINTS ARE:

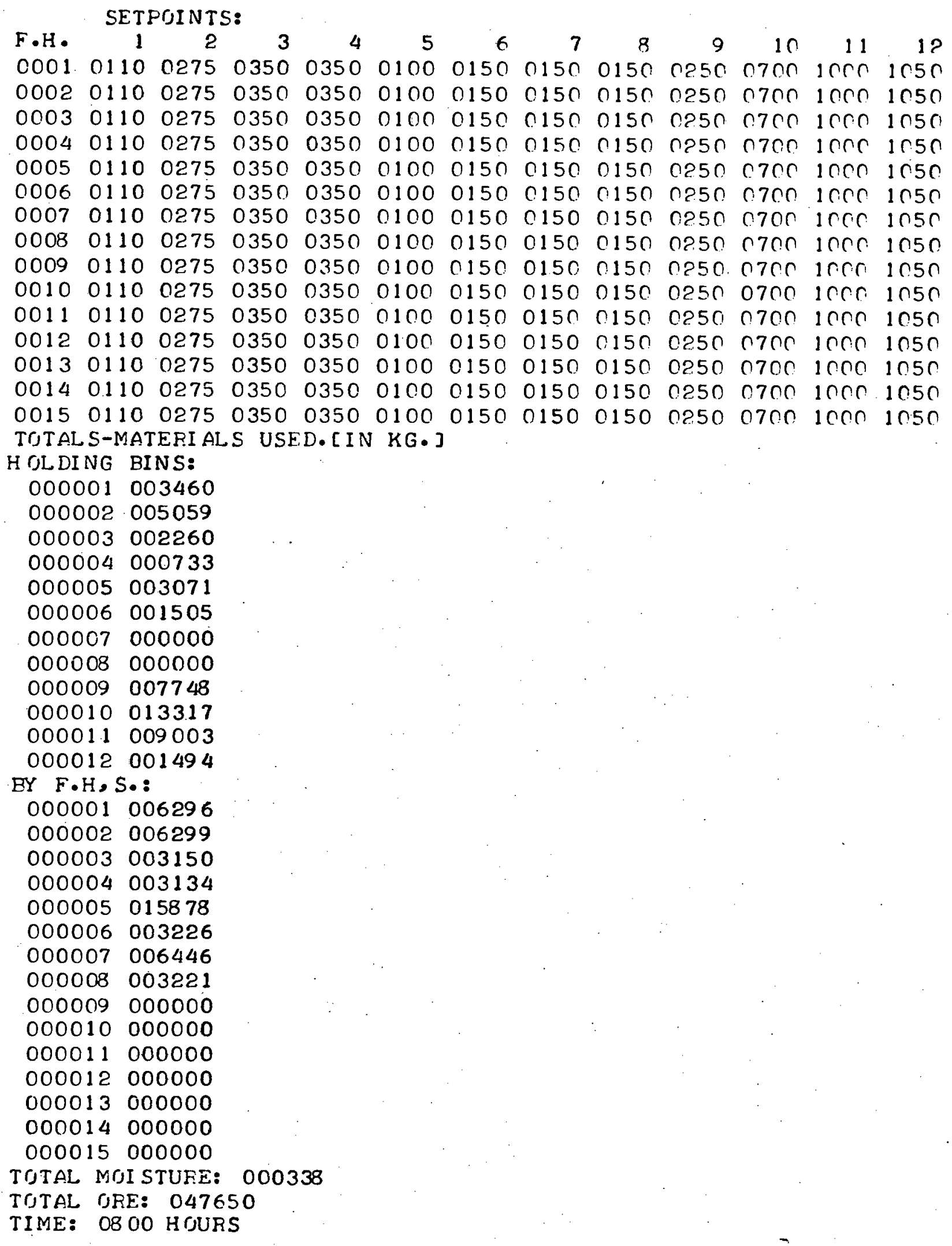

FIGURE 12.10 AN INVENTORY REPORT 
(Chapter 12 - continued)

(c) Entrance

Via a sub-routine call to loc. 3540 .

(d) Description

INVEN can be used either for a shift report in which case the programmer should set 10c. 5063 to zero causing the inventory

parameters to reset. If Ioc. 5063 is non-zero, the inventory parameters will not be reset. Note that the word sizes chosen to store the parameters are such that they can hold any number expected to accumulate within an eight-hour shift period. The progranmer should therefore ensure that INVEN is used with the 1oc. 5063 equal to zero at least once every shift.

\subsection{TO UPDATE THE INVENTORY RECORD}

Sub-routine UPIN

(a) Purpose

To accumulate and store the inventory of raw materials used each time a furnace hopper has been serviced. UPIN therefore prepares the data which is used by INVEN (Chapter 12.7) to make up the data for inventory or shift reports.

(b) Limitations

(i) The quantity of feed mix fed to each furnace hopper and the quantity of raw material fed from each holding bin is accumulated in 16-bit positive words. This gives a maximm of $65,536 \mathrm{~kg}$. of material per shift per accumulator.

(ii) The grand total of all the feed mix consumed is accumulated in a 3-byte word. The routine which outputs numbers is 6 decimal digits long. Therefore a maximu of $999,999 \mathrm{~kg}$ per shift is allowed for.

(c) Program/... 
(Chapter 12 - continued)

(c) Program Location

1oc. $13720-14230$

(d) Entrance

Via a sub-routine call to loc. 13720.

(e) Other Routines Used

(i) Sub-routine SUBR (11.5)

(ii) Sub-routine ADDM (11.1)

(f) Description

Refer to the flow chart, figure 12.11. The quantity of raw material used from each holding bin is calculated as follows:

Let $\mathrm{Hx}$ and $\mathrm{Hx}-1$ be holding bins feeding into the same weighing hopper.

Let SPX and ESPX be the desired setpoint and the error in the desired setpoint.

Then the quantity of raw material fed from $\mathrm{Hx}$ will be:

Quantity from $\mathrm{Hx}=\mathrm{SPX}-\mathrm{ESPX}-$ (quantity from $\mathrm{Hx}-1$ )

SPx - ESPx is stored as an intermediate total called ITx.

The intermediate totals corresponding to the highest setpoint of each weighing hopper is added together to obtain the total feed mix fed to the currently selected furnace hopper.

12.9 TO DETERMINE WHETHER A FURNACE HOPPER REQUIRES ATTENTION:

\section{Sub-routine FEMP}

(a) Purpose

To determine whether the furnace hopper indicated by $B_{R}$ requires service by examining 10c. 6000-6004 (Chapter 12.10). FEMP sets the carry flag to 1 if the selected furnace hopper's feed mix level is low. FEMP sets $D_{R}$ to indicate to which 'filling group' (see Chapter 12.10) the selected furnace hopper belongs.

(b) Program Location

1oc. $14240-14320$

(c) Entrance/..... 
SUBROUTINE UPIN

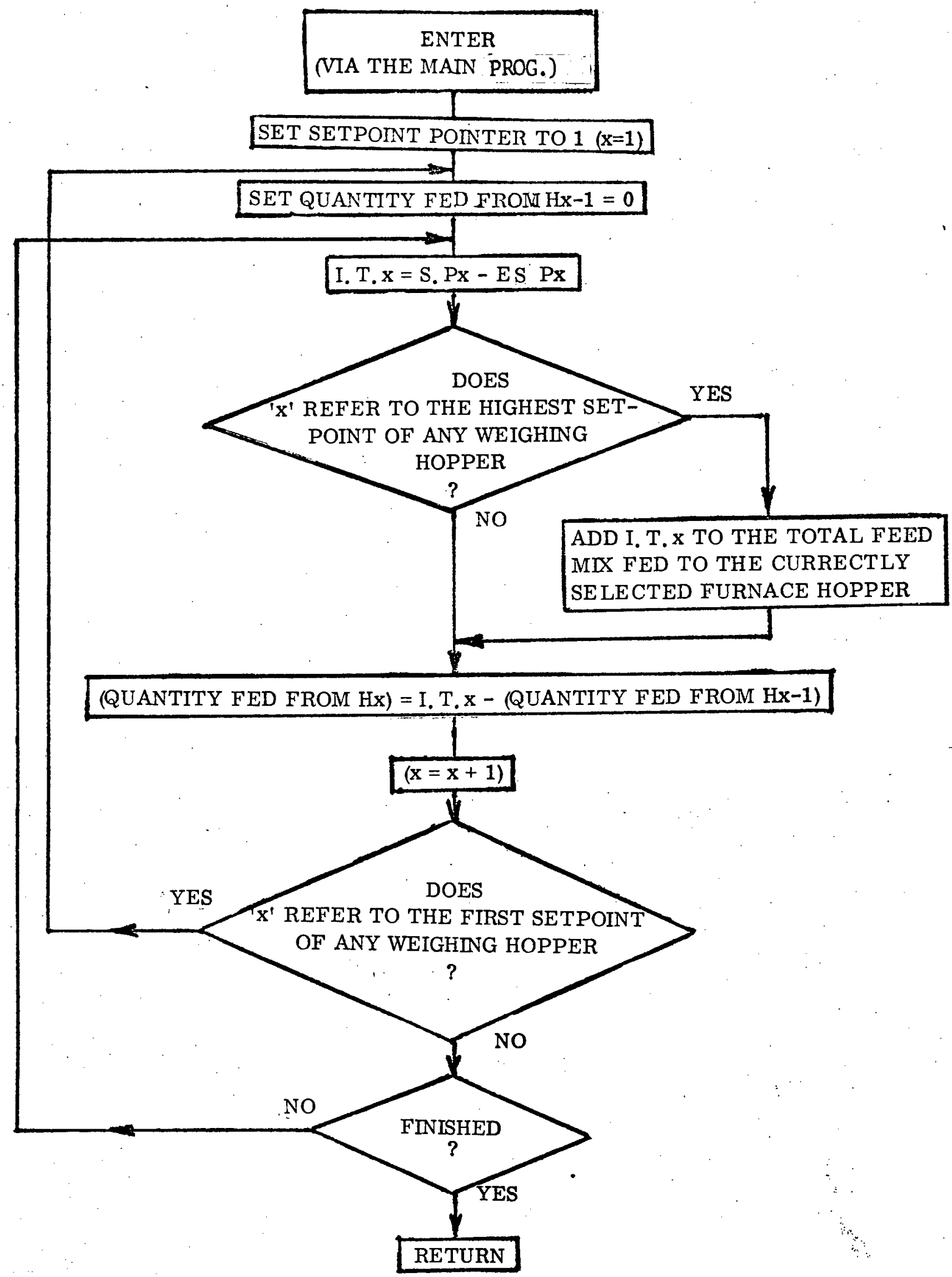

FIGURE 12.11 TO UPDATE THE INVENTORY RECORD 
(Chapter 12 - continued)

(c) Entrance

Via a sub-routine call to loc. 14240.

12.10 TO SELECT THE NEXT FURNACE HOPPER TO BE SERVICED

Sub-routine NEFH

(a) Purpose

To minimise mechanical wear and tear on the conveyor system, it was decided that the fumace hoppers should be grouped into 'filling groups' fed from a common shuttle. NEFH examines $B_{R}$ to see which is the last furnace hopper to have been serviced and uses the value of $D_{R}$ (Chapter 12.9) to see if any furnace hoppers in the same filling group require service. This is used as a first priority selection of which furnace is to be serviced next. The second priority selection is based purely on a numerical cycle. Note that an emergency priority selection mode is used when mamy (more than six) furnace hoppers become empty. (Chapter 12. 11. (e) ii:)

(b) Program Location 1oc. $144330-14370$

(c) Entrance

Via a sub-routine call to loc. 144330 .

\subsection{TO SELECT WHICH FURNACE HOPPER IS TO BE SERVICED}

Routine HOPR.

(a) Purpose

(i) To determine if any furnace hoppers require service.

(ii) To arrange the furnace hoppers which require service into their filling groups and to store these as follows:

loc. 6000 furnace hoppers $1,2,3$ and/or 4 .

loc. 6001 furnace hoppers $5,6,7$, and/or 8 .

1oc. 6002 furnace hoppers $9,10,11$ and/or 12

1oc. 6003 furnace hoppers 13,14 and/or 15 . 
(Chapter 12 - continued)

(iii) To examine and set the 'FURNACE HOPPER SERVICE' flags, thus determining the filling mode which is applicable.

(iv) As a result of (i), (ii) and (iii) above, to select which furnace hopper must then be actually serviced.

(b) Program Location

loc. $11000-11337$
loc. $11456-11574$
loc. $13345-13370$

(c) Entrance

Via a jump to loc. 11000 from the main program. Note that as HOPR is entered via a jump instruction and as HOPR a1so returns to the main program via a jump instruction, it is really part of the main program. It is, however, convenient to consider it separately as a sub-routine.

(d) Other Routines Needed

(i) Sub-routine FEMP (12.9)

(ii) Sub-routine NEFH (12.10)

(iii) Sub-routine SWOP (12.12)

(e) Description

Loc. $6000-6003$ is examined to determine how many furnace hoppers require service. The furnace filling mode is chosen (see the flow chart, Figure 12.12), according to this quantity and the 'FURNACE HOPPER SERVICE FLAGS' adjus ted for:

(i) Filling Mode 1:

Chosen when less than 7 furnace hoppers require ser vice. This is the normal mode (see flow chart, figure 12.12).

(ii) Filling Mode 2.

More than six furnace hoppers require service (see the flow chart, figure 12.13). Mode 2 is a quick filling mode. Whenever it is entered, 2 loads are dumped into each furnace hopper which requires service. 
NTER

VIA A 'JUMP' FROM THE MAIN PROGRAM

READ AND STORE THE STATE OF THE FURNACE : HOPPER LEVEL INDICATORS

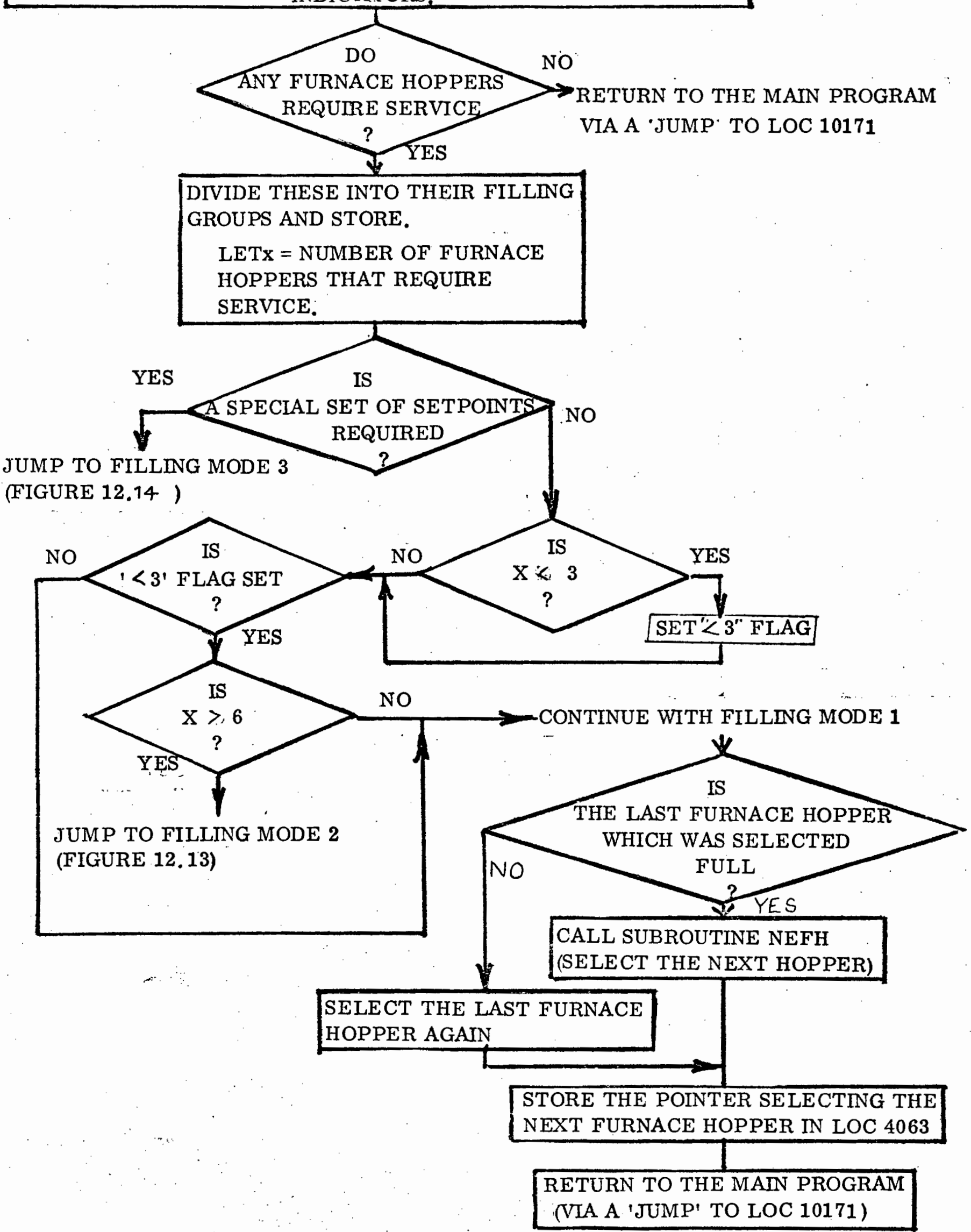

FIGURE 12.12 FILLING MODE 1. 


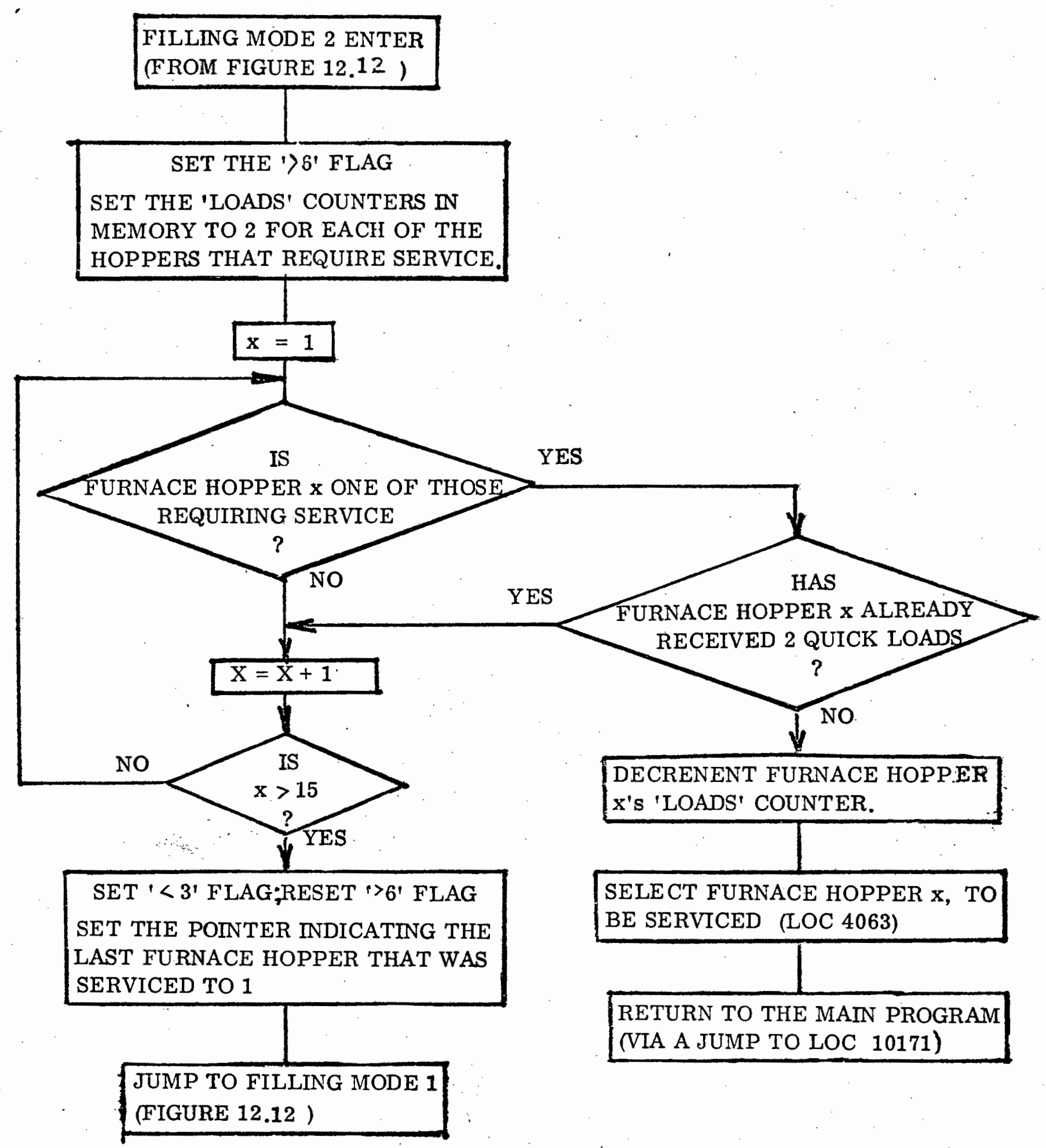

FIGURE 12.13 FILLING MODE 2 


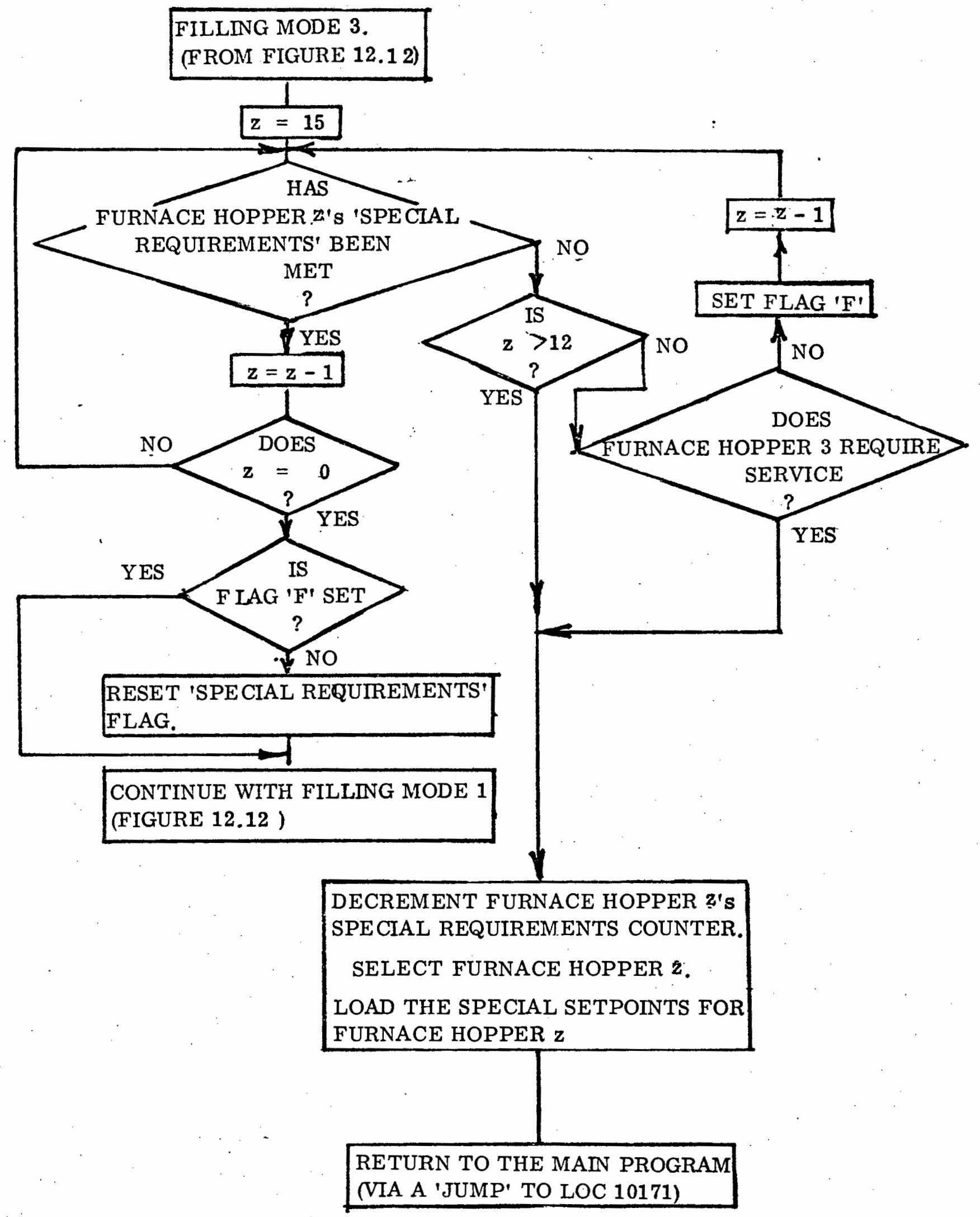

FIGURE 12.14 FILLING MODE 3. 


$$
\text { (Chapter } 12 \text { - continued) }
$$

Control is then passed back to mode 1 and mode 2 cannot be re-entered unless a state has been reached when only two or less furnace hoppers require service. Mode 2 is primarily included so that some buffer capacity of feed mix can be placed into each furnace hopper after the furnace has been tapped. Note the default condition when re-starting the processor will always be with the flag set indicating that two or less furnace hoppers have been emptied.

\section{(iii) Filling Mode 3}

This mode is entered when a flag is set indicating that the operator requires a special set of setpoints for a limited number of batches (12.6.1). (See the flow chart, Figure 12.14).

\subsection{TO LOAD A "SPECIAL RECIPE" FOR A SEEECTED FURNACE HOPPER} Sub-routine SWOP

(a) Purpose

This routine is used to add the differences between the actual recipe and the special recipe to the error factors used for correcting the setpoints before they are used. After the weighing hoppers have been loaded the same routine is used to reverse the above process.

(b) Program Location

$$
\text { 1oc. } 11341-11454
$$

(c) Entrance

Via a sub-routine call to 1oc. 11341 .

(d) Other Routines Used

(i) Sub-routine FISP (12.3)

(ii) Sub-routine COMP (11.4)

\section{(e) Description}

If the flag stored in loc. 6113 is not set, this indicates that SWOP has been used before loading the weighing hoppers and the 'special requirements' are added to the errors in the setpoints. Otherwise, 
(Chapter 12 - continued)

the errors are restored to their previous values.

12.13 COORDINATION OF THE DIFFERENT ROUTINES INTO A CONTROL PROGRAM

THE MAIN PROGRAM

\section{(a) Purpose}

To produce a flexible controlling program. Any major changes in the control philosophy could be accommodated by partly or completely changing the main program. Al1 the sub-programs are designed in such a way that they can fit into any new control philosophy.

(b) Limitations

The main program must operate within the restrictions imposed by the sub-programs and hardware configuration provided.

(c) Error Checks

Many error checks are described in Chapter 12. As discussed in Chapter 10.1, many more possible fault conditions must still be evaluated and, if necessary, included. Some suggestions for possible corrective measures which may be important are given in Section 4.

(d) Program Location

$$
\text { 1oc. } 10000-10470
$$

Note: Provision has been made in this main program to insert new routines and instructions.

(e) Other Routines Used

All the other routines in Chapters 11 and 12 are used directly or indirectly by the main program.

\section{(f) Description}

Refer to all previous descriptions and to the flow chart. Figure 10.1. 
SECTION 4.

FROM PROTOTYPE TO PROCESS. 


\section{CHAPTER 13}

\section{EQUIPMENT FAILURE}

\section{$13.1 \quad$ INTRODUCTION}

As discussed in Chapter 10.1 , it is proposed to evaluate likely equipment failures and develop corrective measures, using the prototype system. This chapter contains a preliminary survey of possible fault conditions and the necessary corrective measures.

It should be noted that at the expense of (1) memory capacity, (2) processor operating speed, (3) software simplicity, and (4) system simplicity, the system designer can specify elaborate and very sophisticated fault detection and correction measures. The prototype only occupies approximately seven kilowords of memory out of a maximum of sixteen, so that the memory capacity is not likely to be exceeded. Although the system contains considerable 'dead-time' periods during which the processor will be idle, faults are most likely to occur during 'active' periods. when operating speed might be critical.

In view of operator and industry inexperience with microprocessor systems, it is desirable to sacrifice as little as possible of the software and systems simplicity. The above factors and also the fact that the furnace hoppers have a large buffer capacity - even when they require service indicate that fault detection and correction should be as simple and as direct as possible.

\subsection{PROCESSOR FAILURE}

To limit possible equipment damage and plant breakdown when the processor fails, it is recommended that the following process interlocks and/or fault detection should exist. (This is excluding the normal over/under voltage trips, etc.)

(i) None/..... 
(i) None of the feeders loading the weighing hoppers should be allowed to operate when any of the feeders emptying them are operating, and vice versa.

(ii) Only one feeder loading a particular weighing hopper must operate at a time.

(iii) None of the feeders loading a weighing hopper must operate when its load cell output has exceeded a maximum value.

(iv) A break in the load cell system must cause the load cell output to indicate its maximm value.

(v) The feeders emptying the weighing hoppers must not operate when the conveyor belt onto which they feed is not running.

(vi) No conveyor should operate unless signals are present, indicating that a specific furnace hopper has been selected and that the plant is therefore in the transportation mode.

\subsection{PROCESS FAILURE}

By using the processor the system designer could utilize the following methods to detect a plant failure.

(a) TIME:

Using the hardware timer (Chapter 6.10) or a time-of-day digital clock, certain events can be timed to ensure that they occur within certain time limits. Areas where this approach can be fruitfully applied are, for instance,

(i) To check that the weighing hoppers are emptied within a certain period. This could indicate the malfunctioning of any of the feeders which empty the weighing hoppers.

(ii) To ensure that the weighing hoppers are loaded within a certain period. This could indicate the malfunctioning of any of the feeders which fill the weighing hoppers.

(iii) To check that the signals indicating that the route to a furnace hopper is working are all present within a certain period. This 
(Chapter 13 - continued)

This would indicate if:

any belt has slipped off;

any belt has 'jammed';

any motor has failed to start;

any motor is rotating too slowly;

a malfunctioning of the shuttle system;

a malfunctioning of a transducer.

(iv) If the operator initiates a commuication routine and for some reason abandons the data terminal, control should automatically be returned to the processor after a certain period.

(b) ALARM RQRT

As explained in Chapter 8.3, an interrupt vector can be used by the plant to initiate a data transfer to the processor. This facility can be used by the process to inform the processor of fault conditions which do not immediately affect the essential operation of the plant and therefore cannot readily be detected. An example of such conditions might be a signal that a motor is overheating or that a holding bin is nearly empty.

(c) CONTINUOUS PROCESS MONITORING

The processor can, whenever it has any idle time, be continuously monitoring the signals from the process. This method could be used while the feed-mix is being transported to a fumace hopper to ensure that the route remains working correctly.

\subsection{CORRECTIVE MEASURES}

(a) During a processor failure, the operator will have to switch over to the manual control system until the processor fault has been corrected.

(b) There are two types of plant failure which should be considered separately:

1) Failures which prevent the system from operating at all and which require immediate attention.

2) Failures which do not affect the operation of the whole process $/ . .$. . 
(Chapter 13 - continued)

process.

Typical corrective measures which can be employed would then be as follows:

(i) When any fault is first detected:

do the necessary operation to prevent any further mishap; inform the operator of the fault;

wait for a response from the operator to confirm the error.

(ii) If the fault is of category (1) above, output an inventory of the materials used since the last shift report and perform an ordered shutdown of the system.

(iii) If the fault is of category (2) above, wait for a further comminication from the operator to indicate how to continue.

The above scheme enables the operator to investigate the error and perhaps by some simple action - like removing a lump of ore sticking into a feeder enable the process to continue normally. The operator can also detect that the error is not due to the plant malfunctioning, but that a transducer is not operating; he can therefore instruct the processor to ignore, for exarmle, a specific 'belt up to speed' signal not being present. 


\title{
CHAPTER 14
}

\section{SYSTEM EVALUATION}

\subsection{INTRODUCTION}

It was found convenient to evaluate the prototype system from the following viewpoints:

\author{
Hardware \\ Software \\ System.
}

\subsection{HARDWARE EVALUATION}

\section{(a) General}

The hardware facilities provided proved to be adequate and there is ample buffer capacity except in the case of the interrupt controller.

(b) The Microprocessor

Doubts had been raised as to whether a micro-computer would prove fast. enough to control the proper loading of the weighing hoppers. The controlling factor is, of course, the operating speed of the 8008 microprocessor. The simulator was operated at speeds in excess of those in a typical plant (see Appendix 5), and the processor was always able successfully to control the weighing hopper 1oading. No overshoot errors occurred which would indicate that a setpoint, having been reached, had not been detected in time. In (5.3) the possibility of upgrading the system to an 8080 micro-processor was raised. It may still prove attractive to change to a faster micro-processor, for reasons discussed in (14.4).

(c) The Interrupt Controller

All eight interrupt vectors provided for have been utilized. See (14.4) for an alternative suggestion as to how the processor should determine that the weighing hoppers are empty. This will free one of the interrupt vectors providing some additional buffer capacity. 
(Chapter 14 - continued)

Experience gained during development of the prototype system suggests that not much is to be accrued in the use of intermpts as a direct means of process control in this type of application. Generally, the processor knows when it expects some process action to happen and can therefore continually monitor the appopriate input port to determine this. Indications are that the areas where interrupt vectors can be put to good use are:

(1) To introduce major branches in the program flow:

(i) To start up the processor:

(ii) To detect a power failure;

(iii) To inform the processor of 'umexpected' operator-initiated commication:

(iv) To inform the processor of 'unexpected' failures or alarm conditions which have occurred.

(2) During Peripheral Commuication:

If all the peripheral controllers are designed so that an intermpt is generated for every commuication action, then the progranmer would be able to write control software without having to include any peripheral 'sense loops'. This implies that disabling of the interrupts for long, continuous periods is undesirable.

(d) The Mimic Pane1

The mimic panel proved to be of great value. A possible improvement would be to have i.e.d. displays indicating which furnace hopper is to be serviced, i.e. the position of the shuttle above that furnace hopper. At present, this can only be determined when the 1.e.d., indicating that it is being charged with feed mix, operates.

(e) The Operator's Control Pane1

The facilities provided seem to be adequate but this, as with point (d) above, can only be determined by introducing the prototype system to operators of a real plant.

(f) Current/.... 
(Chapter 14 - continued)

(f) Current Processing Phase

If the present control strategy is adhered to, namely, that long periods of time exist during which the interrupts are disabled and no peripherals will respond to external commmications requests, then it is strongly recommended that an external hardware flag register, under program control, exist to indicate to other devices, i.e. a controlling computer or to the operator (via 1.e.d. displays), which processing phase is being executed.

\subsection{SOFTWARE}

In Chapter 10.1, the objectives of the software for the prototype were stated. These were to:

Evaluate the hardware;

Evaluate the programing aids;

Develop control strategy.

The hardware evaluation was done in Chapter 14.2. The control strategy will be discussed in Chapter 14.4 .

An Evaluation of the Programming Aids

The hardware programing aids proved to be useful and functional. The system which loads the processor from the VARIAN minicomputer proved to be particularly useful. The progranmer could use the facilities provided by the VARIAN to:

- Store programs on paper tape;

- Change programs using VARIAN's debug techniques;

- Load and re-load the RAM memory of the processor easily and quickly:

The Direct Memory access loader and display was invaluable once the memory was loaded for the following applications: 
(Chapter 14 - continued)

- Checking programs;

- Changing locations in memory;

- Checking memory locations when stepping through a program to debug it.

A device which would most probably be needed for debugging the system when it is being installed on the plant and after its control program has been committed to ROM would be a hardware 'program breakpoint'. This is a device which would inhibit the 'ready' signal whenever the address bus compares with an address specified via front panel switches. This would enable the field engineer to insert breakpoints in the control flow of the program during the system checkout phase. Although a similar function was implemented using software on the prototype - remember that random excess memory was used - it would have been useful during program development also.

All the software was develpped using the $8008^{\prime}$ 's machine coded instructions. No complex calculations were required and the control functions are relatively simple. A process control orientated macro-assembler with the utility routines mentioned in Chapter 11 plus some general control routines available as some of its functions within the instruction set, would considerably reduce the programming time and complexity in this type of application.

It is doubtful if any conventional compilers or translators would have provided the programmer with the same degree of input/output control and freedom of memory allocation which were considered invaluable during the machine-coded program development.

\subsection{SYSTEMS EVALUATION}

The problem of whether the system would be fast enough to execute certain control functions led to the development of initial control strategy in which interrupts are disabled for long continuous periods. External devices required to initiate communication with the processor therefore have to 'wait' for considerable periods during certain processing phases. The software techniques to implement 
this type of strategy is simpler than those for a conventional, "time-shared system"which will, for instance, give the operator the impression that he has continual access to the processor. If the present system is adhered to, the hardware flag register mentioned in Chapter 14.2 (f) will become a necessity. It will 'tell' the operator when the teletype will 'respond' to his 'requests' and will inform other devices (1ike a controlling computer) when access to the processor is possible.

Initial investigation suggests, however, that the $8008 \mathrm{micro-}$ porcessor is so fast that the interrupts need never be disabled after the system is initialized. This would enable the programmer to change, for example, the routines communicating with the teletype so that interrupts from the teletype interface would cause immediate control actions rather than just setting a flag in memory.

At present, an interrupt is generated to the processor when all three weighing hoppers are empty. This system has the following disadvantages:

(i) It:will be difficult for external hardware to tell when a weighing hopper is empty because of possible taring errors.

(ii) It will be difficult to pinpoint an error while the hoppers are being emptied.

A preferable scheme is to let the processor periodically monitor the load cell: outputs when the feeders emptying the feeding hoppers are operating. This would enable the individual monitoring of the discharge rates, and thereby facilitate error detection. This also releases one of the interrupt vectors. 
(Chapter 14 - continued)

\subsection{CONCLUSION}

The microprocessor provides the control systems designer with an exciting alternative in applications where computer contro1 is desirable. In many such applications, however, conventional mini-computer based systems would be too expensive or too elaborate - like cracking a nut with a sledge hammer. In view of its low price, flexibility and miniature size, the microprocessor is opening up new application areas which previously were not considered.

The features comprising the micro-processor based controller described in this thesis were designed with a specific application in mind. However, only the timing range of the 'timer' and the software developed have any specific characteristics relating to the batch-weighing and feed-mix transportation system of an arc furnace. The fact that such a low cost system (no higher than R1000 in hardware costs) would easily adapt to many entirely different systems underlines the great flexibility of microprocessor based systems.

A flexible, intelligent controller has been developed. The practicability of applying a micro-processor in such an original application as this has been established. Furthermore, the micro-processor based system produces a practical control tool, with a degree of accuracy and with inventory logging facilities which are far cheaper than any offered by competitive analog or digital systems. 


\section{BIBLIOGRAPHY,}

1. ASPHAUG, B. and HEMPEL A. : A computer controlled batch weighing system for electric smelting furnaces.

* Paper. ELKEM-SPIGERVERKET. NORWAY. 1972.

2. NIM TECHNICAL REPORT : The computer control of a ferrochromium furnace.

* REPORT NO. 1676.

3. ARNESEN A. G. and ASPHAUG, B. : Computer control of a 39 MVA Electric Furnace for the Production of High Carbon Ferromanganese.

* Proc. El. Furn. Conf. 1972: Special Arc Session. pp. 67-77.

4. CASCIANTI. R.A., FRANSON P.D. and WILBEM, W. L. : Computer Control of Submerged Arc Furnace.

* Proc. El. Furn. Conf. 1967. Vol. 25, Chicago, pp. 68-71.

5. SPAL. J. : Digital computers in the control of electrosteel making processes.

* Elektrowárme Intermational 32 (1974) B 5, Oktober.

6. MICROCOMPUTER TECHNIQUES INC. : Microprocessor scorecard.

* New Logic Notebook. Sept. 1974. Vol. 1, No. 1.

7. GLADSTONE. BRUCE : Designing with microprocessors instead of wired logic asks more of designers.

* Electronics (Oct. 11. 1973).

8. NELSON. ¿̇. R. : Microprocessor Applications

* Computer (Aug., 1974).

9. NELSON, D.R. : Microprocessor Architecture

* Computer (July, 1974).

10. SABA, M. M. and GRIMES, J.D. : Microprocessors : A component for all seasons.

* Wescon 1974 (A Tekronix technical reprint).

11. LEWANDOWSKI, $R_{0}$ : Preparation : The key to success with microprocessors.

SANTONI, A. : Microprocessors await the call.

*. Electronics (Mar. 20, 1975).

12. DAVIS. S. : A fresh view of Mini - and Microcomputers.

* Computer Design (May, 1974). 
Bibliography - Continued.

13. BAILEY, S. J. : Microprocessor : Candidate for distributed computing control.

* Control Engineering (Mar., 1974.)

14. DAVIS, S. : Selection and Applications of Semiconductor Memories.

* Computer Design (Jan., 1974)

15. SPECIAL REPORT : Logic

* Electronics (Feb., 21, 1974.)

16. GARRET, L.S. : Integrated-circuit digital logic families.

* IEEE Spectrum (Oct., Nov., Dec., 1970.)

17. THE TTL DATA BOOK

* Texas Instruments (1973)

18. INTEL DATA CATALOG. (Oct., 1973.)

19. THE INTEL MEMORY DESIGN HANDBOOK. (Aug., 1973.) 
146

A P P E N I CES 


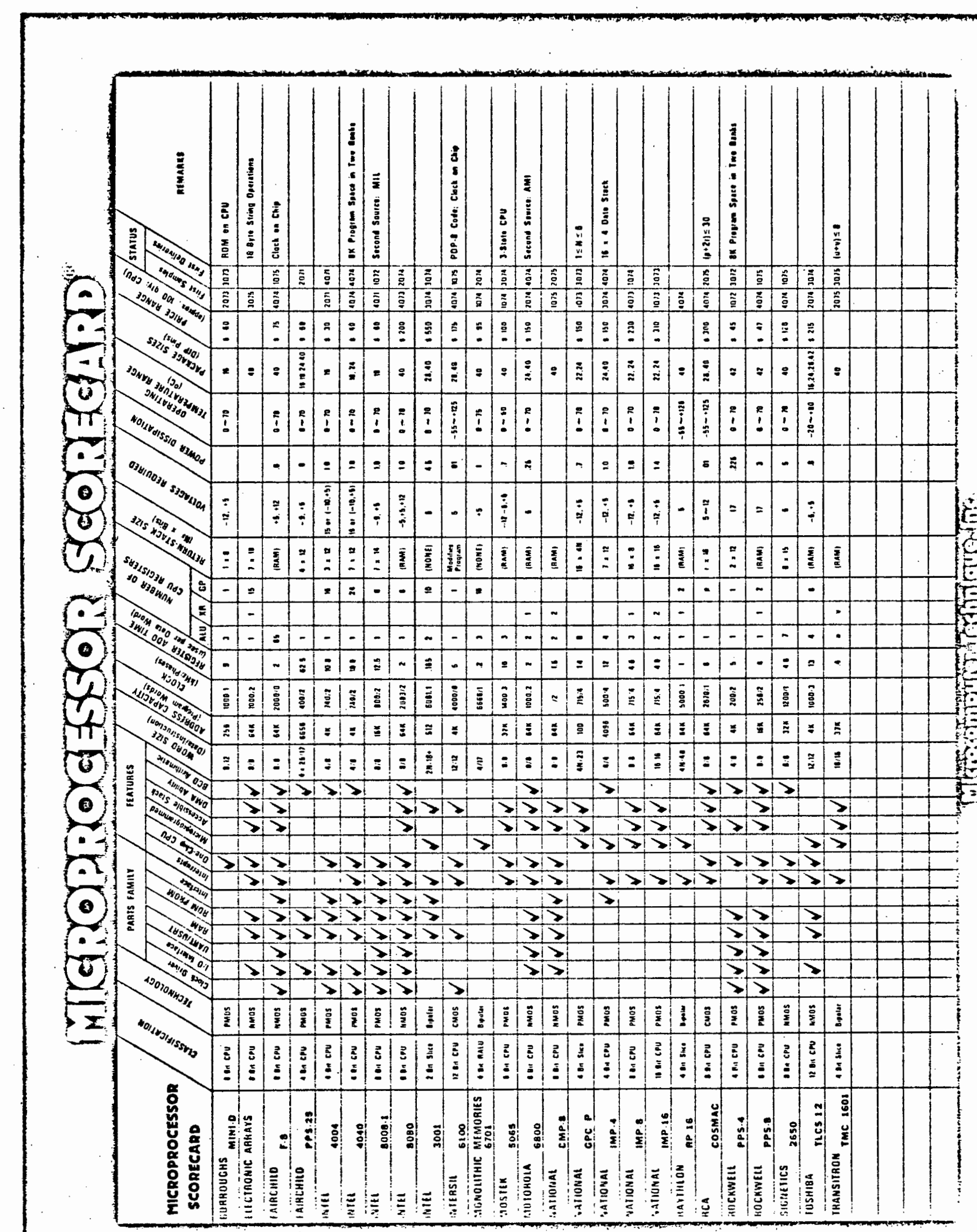


APPENDIX $2-8008$ DATA

\section{INTRODUCTION}

The 8008 is a single chip MOS 8 -bit parallel central processor unit for the MCS 8 micro computer system. A micro computer system is formed when the 8008 is interfaced with any type or speed standard semiconductor memory up to 16K 8-bit words. Examples are INTEL's 1101, 1103, 2102 (RAMs), 1302, 1602A, 1702A (ROMis), 1404, 2405 (Shift Registers).

The processor communicates over an 8-bit data and address bus $\left(D_{0}\right.$ through $\left.D_{7}\right)$ and uses two input leads (READY and INTERRUPT) and four output leads $\left(S_{0}, S_{1}, S_{2}\right.$ and $\left.S y n c\right)$ for control. Time multiplexing of the data bus allows control information, 14 bit addresses, and data to be transmitted between the CPU and external memory.

This CPU contains six 8-bit data registers, an 8-bit accumulator, two 8-bit temporary registers, four flag bits, and an 8-bit parallel binary arithmetic unit which implements addition, subtraction, and logical operations. A memory stack containing a 14 -bit program counter and seven 14-bit words is used internally to store program and subroutine addresses. The 14-bit address permits the direct addressing of $16 \mathrm{~K}$ words of memory (any mix of RAM, ROM or S.R.).

The control portion of the chip contains logic to implement a variety of register transfer, arithmetic control, and logical instructions. Most instructions are coded in one byte (8 bits); data immediate instructions use two bytes; jump instructions utilize three bytes. Operating with a $500 \mathrm{kHz}$ clock, the $8008 \mathrm{CPU}$ executes non-memory referencing instructions in 20 microseconds. A selected device, the 8008-1, executes non-memory referencing instructions in 12.5 microseconds when operating from an $800 \mathrm{kHz}$ clock.

All inputs (including clocks) are TTL compatible and all outputs are low-power TTL compatible:

The instruction set of the 8008 consists of 48 instructions including data manipulation, binary arithmetic, and jump to subroutine.

The normal program flow of the 8008 may be interrupted through the use of the "INTERRUPT" control line. This allows the servicing of slow $1 / 0$ peripheral devices while also executing the main program.

The "READY" command line synchronizes the 8008 to the memory cycle allowing any type or speed of semiconductor memory to be used.

STATE and SYNC outputs indicate the state of the processor at any time in the instruction cycle. 


\section{PROCESSOR TIMING}

The 8008 -is a complete central processing unit intended for use in any arithmetic, control, or decision. making system. The internal organization is centered around an 8-bit internal data bus. All communication within the processor and with external components occurs on this bus in the form of 8-bit bytes of address, instruction or data. (Refer to the accompanying block diagram for the relationship of all of the internal elements of the processor to each other and to the data bus.) For the MCS 8 a logic " 1 " is defined as a high level and a logic " 0 " is defined as a low level.

\section{A. State Control Coding}

The processor controls the use of the data bus and determines whether it will be sending or receiving data. State signals $S_{0}, S_{1}$, and $S_{2}$, along with SYNC inform the peripheral circuitry of the state of the processor. A table of the binary state codes and the designated state names is shown below.

\begin{tabular}{|c|c|c|c|l|}
\hline$S_{0}$ & $s_{1}$ & $s_{2}$ & STATE \\
\hline 0 & 1 & 0 & T1 \\
0 & 1 & 1 & T11 \\
0 & 0 & 1 & T2 \\
0 & 0 & 0 & WAIT \\
1 & 0 & 0 & T3 \\
1 & 1 & 0 & STOPPED \\
1 & 1 & 1 & T4 \\
1 & 0 & 1 & T5 \\
\hline
\end{tabular}

\section{B. Timing}

Typically, a machine cycle consists of five states, two states in which an address is sent to memory (T1 and T2), one for the instruction or data fetch (T3), and two states for the execution of the instruction (T4 and T5). If the processor is used with slow memories, the READY line synchronizes the processor with the memories. When the memories are not available for either sending or receiving data, the processor goes into the WAIT state. The accompanying diagram illustrates the processor activity during a single-cycle.

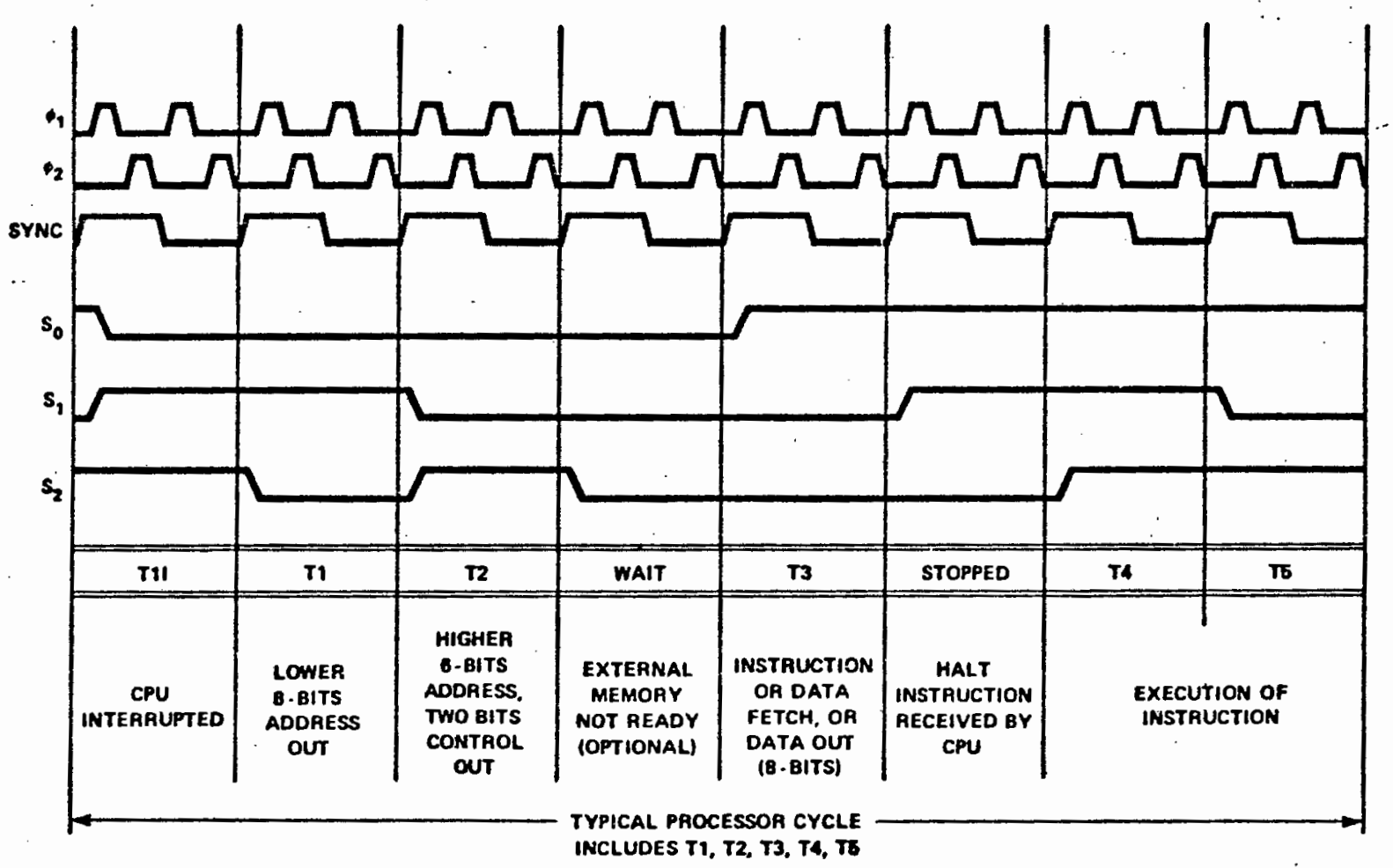

Figure 1. Basic 8008 Instruction Cycle 
The receipt of an INTERRUPT is acknowledged by the T11. When the processor has been interrupted, this state replaces T1. A READY is acknowledged by T3. The STOPPED state acknowledges the receipt of a HALT instruction.

Many of the instructions for the 8008 are multi-cycle and do not require the two execution states, T4 and T5. As a result, these states are omitted when they are not needed and the 8008 operates asynchronously with respect to the cycle length. The external state transition is shown below. Note that the WAIT state and the STOPPED may be indefinite in length (each of these states will be $2 \mathrm{n}$ clock periods). The use of READY and INTERRUPT with regard to these states will be explained later.

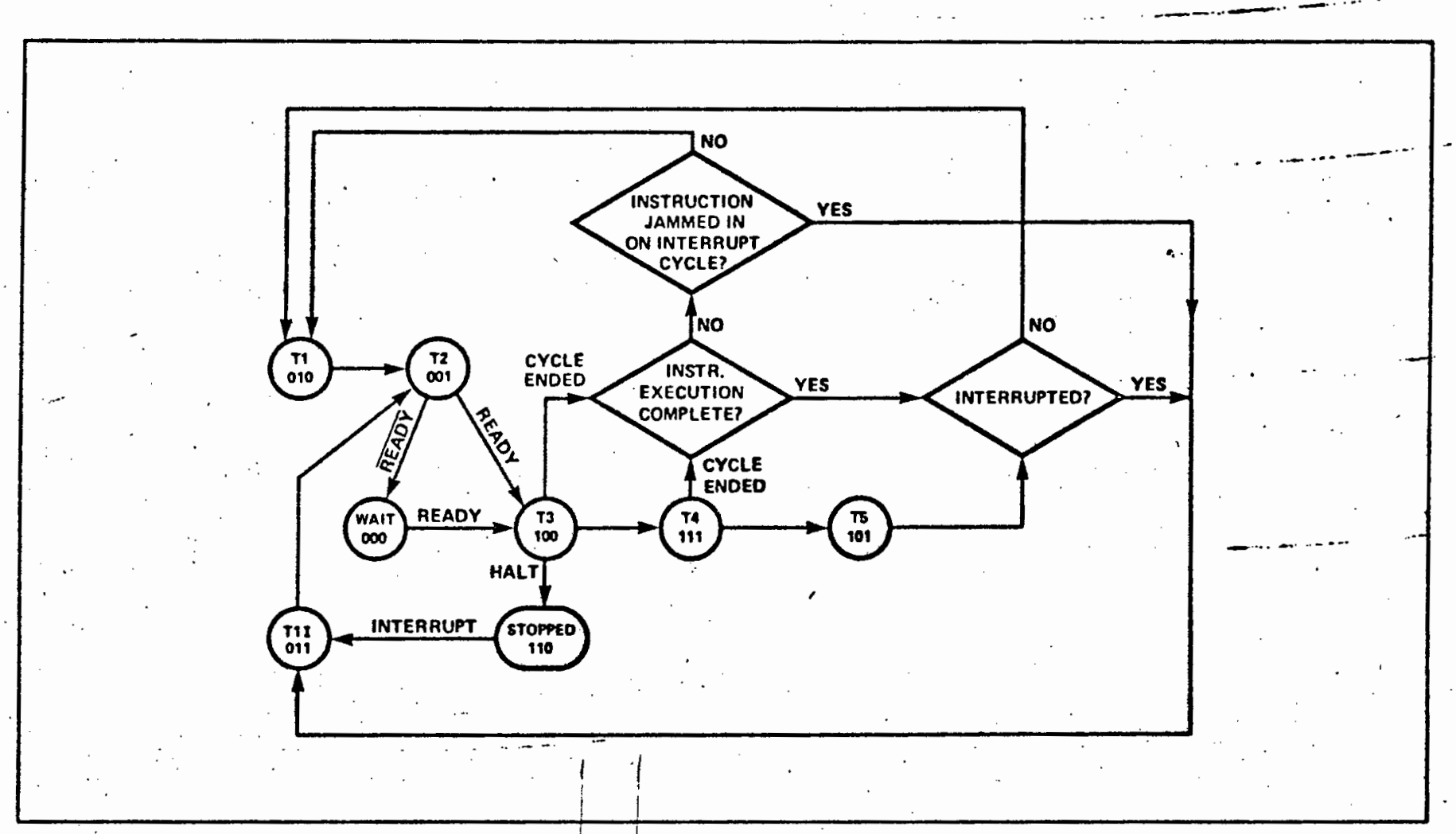

Figure 2. CP.U State Transition Diagram

\section{Cycle Control Coding}

As previously noted, instructions for the 8008 require one, two, or three machine cycles for complete execution. The first cycle is always an instruction fetch cycle (PCI). The second and third cycles are for data reading (PCR), data writing (PCW), or I/O operations (PCC).

The cycle types are coded with two bits, $D_{6}$ and $D_{7}$, and are only present on the data bus during $T 2$.

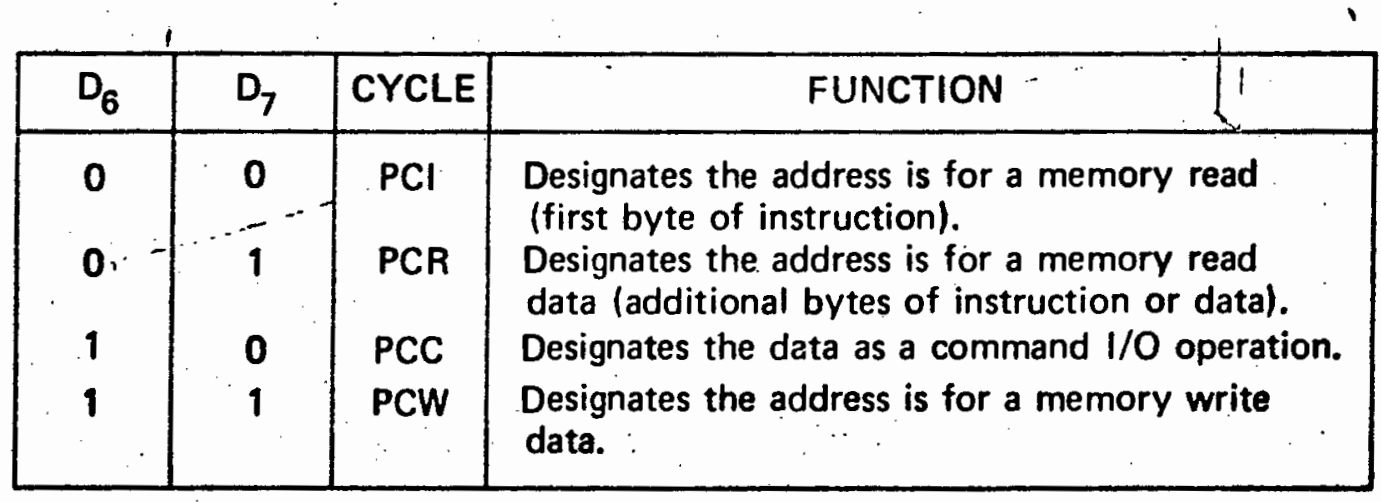




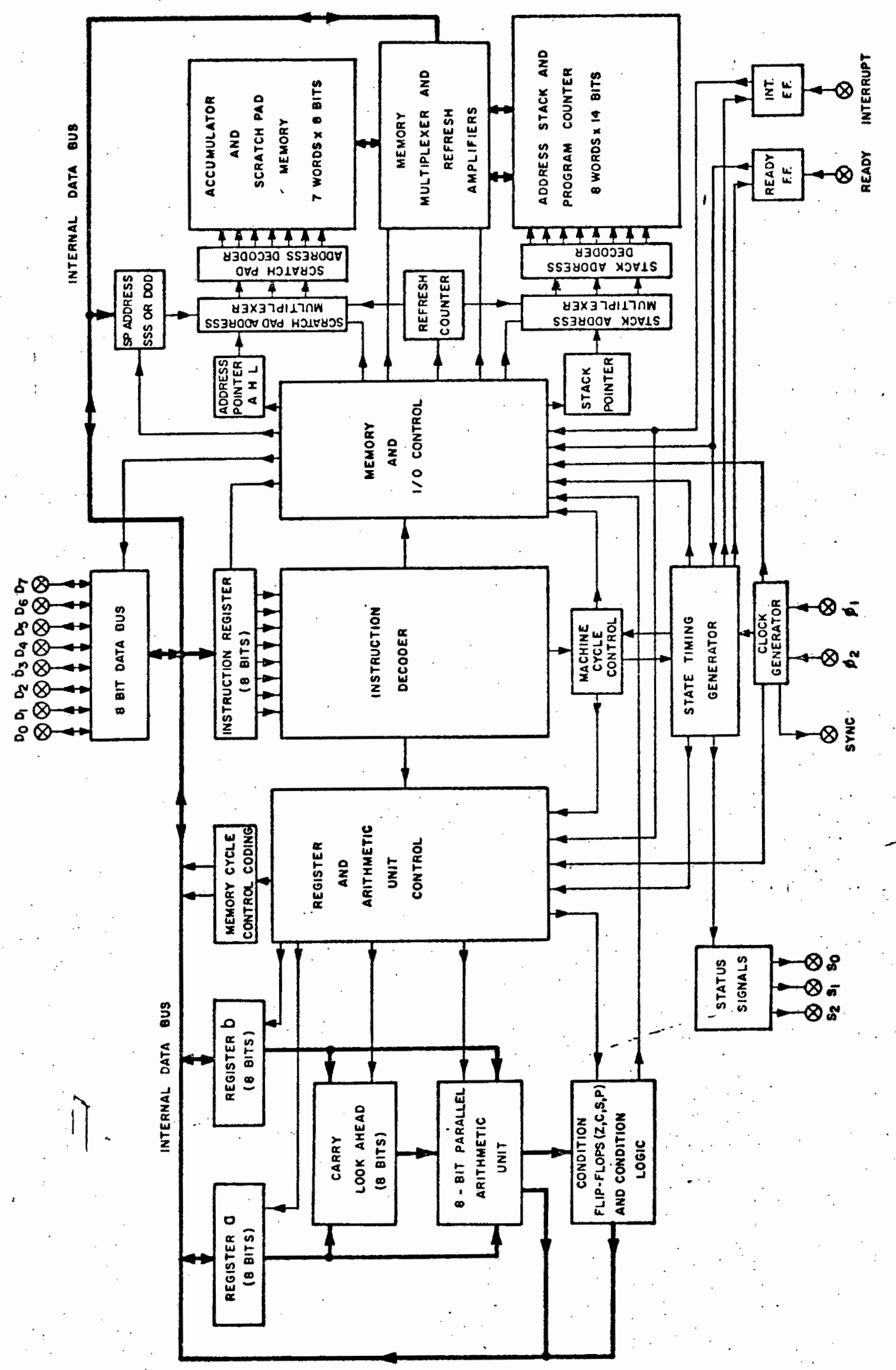

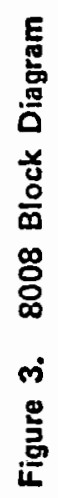




\section{BASIC FUNCTIONAL BLOCKS}

The four basic functional blocks of this Intel processor are the instruction register, memory, arithmeticlogic unit, and 1/O buffers. They communicate with each other over the internal 8-bit data bus.

\section{A. Instruction Register and Control}

The instruction register is the heart of all processor control. Instructions are fetched from memory, stored in the instruction register, and decoded for control of both the memories and the ALU. Since instruction executions do not all require the same number of states, the instruction decoder also controls the state transitions.

\section{B. Memory}

Two separate dynamic memories are used in the 8008, the pushdown address stack and a scratch pad: These internal memories are automatically refreshed by each WAIT, T3, and STOPPED state. In the worst case the memories are completely refreshed every eighty clock periods.

\section{Address Stack}

The address stack contains eight 14-bit registers providing storage for eight lower and six higher order address bits in each register. One register is used as the program counter (storing the effective address) and the other seven permit address storage for nesting of subroutines up to seven levels. The stack automatically stores the content of the program counter upon the execution of a CALL instruction and automatically restores the program counter upon the execution of a RETURN. The CALLs may be nested and the registers of the stack are used as last in/first out pushdown stack. A three-bit address pointer is used to designate the present location of the program counter. When the capacity of the stack is exceeded the address pointer recycles and the content of the lowest level register is destroyed. The program counter is incremented immediately after the lower order address bits are sent out. The higher order address bits are sent out at $T 2$ and then incremented if a carry resulted from T1. The 14-bit program counter provides direct addressing of $16 \mathrm{~K}$ bytes of memory. Through the use of an $1 / 0$ instruction for bank switching, memory may be indefinitely expanded.

\section{Scratch Pad Memory or Index Registers}

The scratch pad contains the accumulator (A register) and six additional 8-bit registers (B, C, D, $E, H, L$ ). All arithmetic operations use the accumulator as one of the operands. All registers are independent and may be used for temporary storage. In the case of instructions which require operations with a register in external 'memory, scratch pad registers $H \& L$ provide indirect addressing capability; register $\mathrm{L}$ contains the eight lower order bits of address and register $\mathrm{H}$ contains the six higher order bits of address (in this case bit 6 and bit 7 are "don't cares").

\section{Arithmetic/Logic Unit (ALU)}

All arithmetic and logical operations (ADD, ADD with carry, SUBTRACT, SUBTRACT with borrow, AND, EXCLUSIVE OR, OR, COMPARE, INCREMENT, DECREMENT) are carried out in the 8-bit parallef arithmetic unit which includes carry-look-ahead logic. Two temporary resisters, register "a" and register " $b$ ", are used to store the accumulator and operand for ALU operations. In addition, they are used for temporary address and data storage during intra-processor transfers. Four control bits, carry flip-flop (c) , zero flip-flop (z), sign flip-flop (s), and parity flip-flop (p), are set as the result of each arithmetic and logical operation. These bits provide conditional branching capability through CALL. JUMP, or RETURN on condition instructions. In addition, the carry bit provides the ability to do mul . tiple precision binary arithmetic.

\section{I/O Buffer}

This buffer is the only link between the processor and the rest of the system. Each of the eight buffers is bi-directional and is under control of the instruction register and state timing. Each of the buffers is low power $T T L$ compatible on the output and $T L$ compatible on the input. 


\section{BASIC INSTRUCTION SET}

The following section presents the basic instruction set of the 8008 .

\section{A. Data and Instruction Formats}

Data in the 8008 is stored in the form of 8 -bit binary integers. All data transfers to the system data bus will be in the same format.

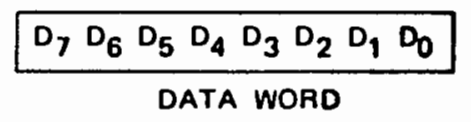

The program instructions may be one, two, or three bytes in length. Multiple byte instructions must be stored in successive words in program memory. The instruction formats then depend on the particular operation executed.

\begin{tabular}{|c|c|}
\hline \multicolumn{2}{|l|}{ One Byte Instructions } \\
\hline 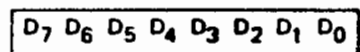 & OP CODE \\
\hline Two Bvie Instructions & \\
\hline 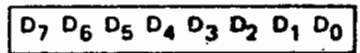 & OP CODE \\
\hline $\begin{array}{llllllllll}D_{7} & D_{6} & D_{5} & D_{4} & D_{3} & D_{2} & D_{1} & D_{0}\end{array}$ & OPERAND \\
\hline Three Byte Imseructions & \\
\hline$D_{7} D_{6} D_{5} D_{4} D_{3} D_{2} D_{1} D_{0}$ & OP COOE \\
\hline$D_{7} D_{6} D_{5} D_{4} D_{3} D_{2} D_{1} D_{0}$ & LOW ADDRESS \\
\hline 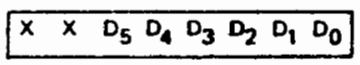 & \\
\hline
\end{tabular}

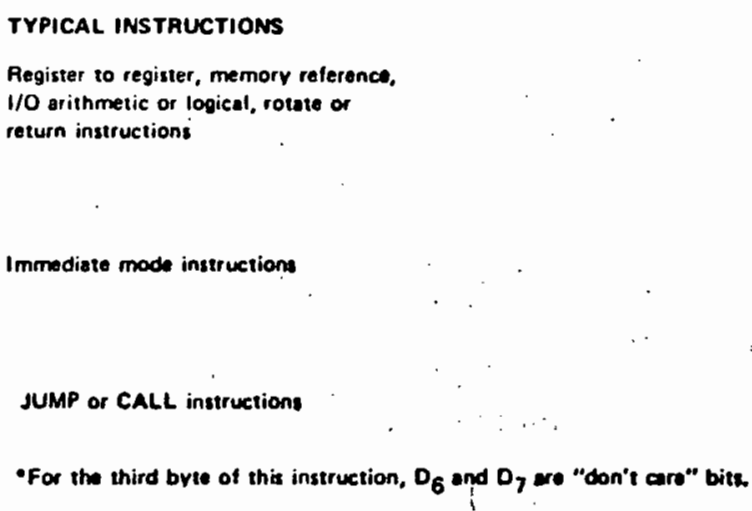

For the MCS-8 a logic " 1 " is defined as a high level and a logic " 0 " is defined as a low level.

\section{B. Summary of Processor Instructions}

\section{Index Register Instructions}

The load instructions do not affect the flag flip-flops. The increment and decrement instructions affect all flipflops except the carry.

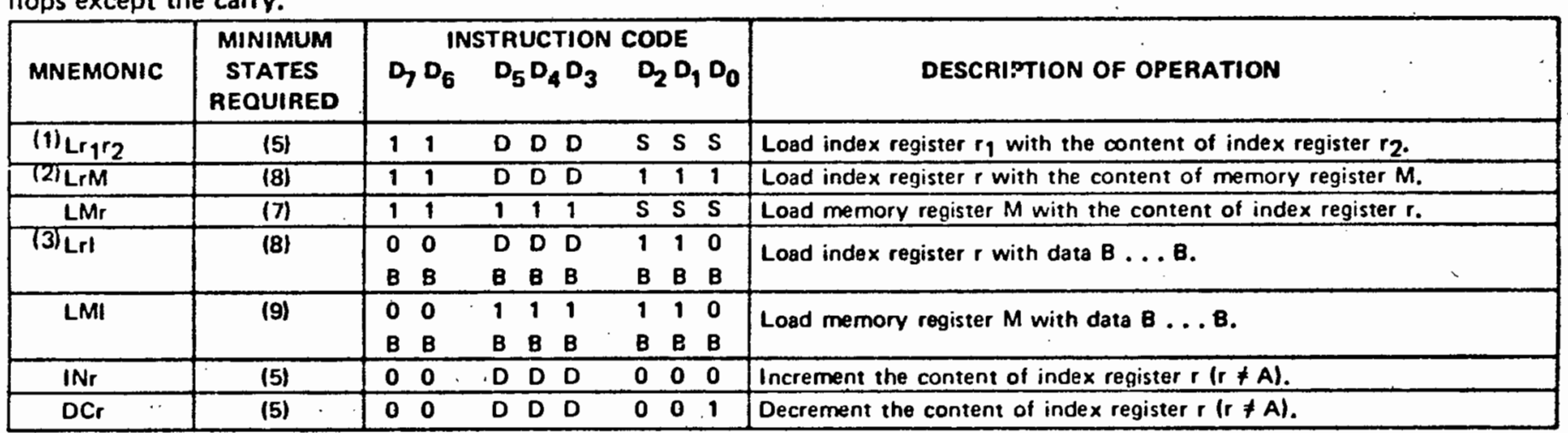

\section{Accumulator Group Instructions}

The result of the ALU instructions affect all of the flag flip-flops. The rotate instructions affect only the carry flip-flop.

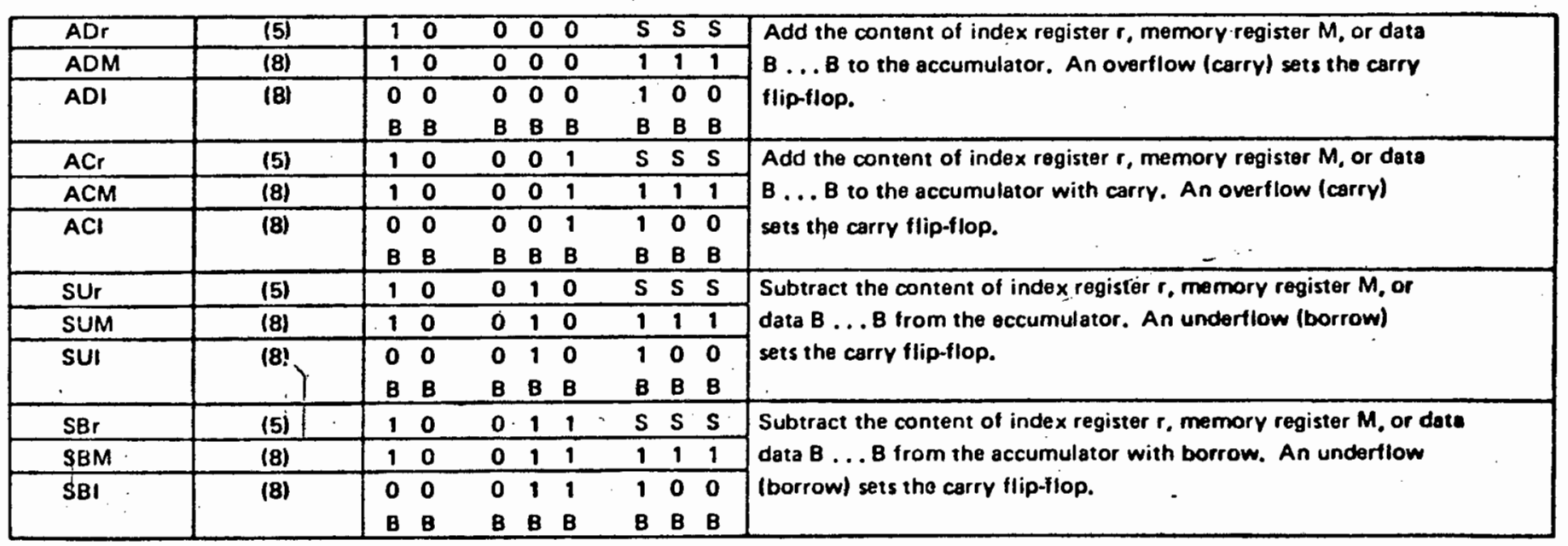




\begin{tabular}{|c|c|c|c|c|c|c|}
\hline \multirow{3}{*}{$\begin{array}{l}\text { MNEMONIC } \\
\text { NDr }\end{array}$} & \multirow{2}{*}{$\begin{array}{r}\text { MINIMUM } \\
\text { STATES } \\
\text { REQUIRED }\end{array}$} & \multicolumn{4}{|c|}{ INSTRUCTION CODE } & \multirow[b]{2}{*}{ DESCAIPTION OF OPERATION } \\
\hline & & $D_{7} D_{6}$ & \multicolumn{2}{|c|}{$D_{5} D_{4} D_{3}$} & $D_{2} D_{1} D_{0}$ & \\
\hline & (5) & 10 & 10 & 0 & $S$ S S & \multirow{3}{*}{$\begin{array}{l}\text { Compute the logical AND of the content of index register } r \text {, } \\
\text { memory register } M \text {, or data } B \ldots \text { with the Bccumulator. }\end{array}$} \\
\hline NDM & (8) & 10 & 10 & $\mathbf{0}$ & 111 & \\
\hline NDI & (8) & $\begin{array}{ll}0 & 0 \\
B & B \\
\end{array}$ & $\begin{array}{ll}1 & 0 \\
B & B \\
\end{array}$ & & $\begin{array}{lll} & 0 & 0 \\
B & B & B \\
\end{array}$ & \\
\hline$X R_{r}$ & (5) & 10 & 10 & 1 & S S S & \multirow{3}{*}{$\begin{array}{l}\text { Compute the EXCLUSIVE OR of the content of index register } \\
\text { r. memory register } M \text {, or dats B . . B with the occumulator. }\end{array}$} \\
\hline XRM & (8) & 10 & 10 & 1 & 111 & \\
\hline $\mathbf{X R !}$ & (8) & $\begin{array}{ll}0 & 0 \\
B & B \\
\end{array}$ & $\begin{array}{ll}1 & O \\
B & B \\
\end{array}$ & $\begin{array}{l}1 \\
8\end{array}$ & \begin{tabular}{lll|} 
& $\mathbf{O}$ & $\mathbf{0}$ \\
$\mathrm{B}$ & $\mathrm{B}$ & $\mathrm{B}$ \\
\end{tabular} & \\
\hline ORr & (5) & 10 & 11 & 0 & $S S S$ & \multirow{3}{*}{$\begin{array}{l}\text { Compute the INCLUSIVE OR of the content of index register } \\
r \text {, memory register } m \text {, or date B . B with the accumulator. }\end{array}$} \\
\hline ORM & (8) & 10 & 11 & 0 & 111 & \\
\hline ORI & (8) & $\begin{array}{ll}\mathbf{0} & \mathbf{0} \\
\mathrm{B} & \mathrm{B} \\
\end{array}$ & & $\begin{array}{l}\mathbf{0} \\
\mathbf{B}\end{array}$ & \begin{tabular}{lll|} 
& 0 & 0 \\
$B$ & $B$ & $B$ \\
\end{tabular} & \\
\hline $\mathrm{CPr}$ & (5) & 10 & 11 & 1 & $\begin{array}{lll} & 5 & 5 \\
\end{array}$ & \multirow{3}{*}{$\begin{array}{l}\text { Compare the content of index register } r \text {, memory register } M \text {. } \\
\text { or data } B \ldots B \text { with the accumulator. The content of the } \\
\text { accumulator is unchanged. }\end{array}$} \\
\hline CPM & 181 & 10 & 11 & 1 & 111 & \\
\hline CPI & (8) & $\begin{array}{ll}\mathbf{O} & 0 \\
\mathrm{~B} & \mathrm{~B}\end{array}$ & $\begin{array}{ll}1 & 1 \\
B & B\end{array}$ & $\begin{array}{l}1 \\
B\end{array}$ & $\begin{array}{lll}1 & 0 & 0 \\
B & B & B\end{array}$ & \\
\hline RLC & (5) & $0 \quad 0$ & 00 & 0 & $0 \quad 1 \quad 0$ & Rotate the content of the accumulator left. \\
\hline RRC & (5) & 0.0 & 00 & 1 & $\begin{array}{lll}0 & 1 & 0 \\
\end{array}$ & Rotate the content of the accumulator right. \\
\hline RAL & (5) & 00 & 01 & 0 & $0 \quad 10$ & Rotate the content of the accumulator left through the carry. \\
\hline RAR & (5) & 00 & 01 & 1 & 010 & Rotate the content of the accumulator right through the carry. \\
\hline
\end{tabular}

Program Counter and Stack Control Instructions

\begin{tabular}{|c|c|c|c|c|c|c|}
\hline (4) JMP. & (11) & $\begin{array}{ll}0 & 1 \\
B_{2} & B_{2} \\
X & X\end{array}$ & $\begin{array}{lll}X & x & x \\
B_{2} & B_{2} & B_{2} \\
B_{3} & B_{3} & B_{3}\end{array}$ & & 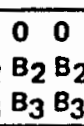 & Unconditionally jump to memory address $\mathrm{B}_{3} \ldots \mathrm{B}_{3} \mathrm{~B}_{2} \ldots \mathrm{B}_{2}$. \\
\hline (5) JFC & $(9$ or 11$)$ & $\begin{array}{ll}0 & 1 \\
B_{2} & B_{2} \\
X & X\end{array}$ & 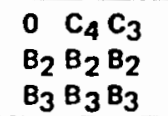 & $\begin{array}{l}\mathbf{O} \\
\mathbf{B}_{2} \\
\mathrm{~B}_{3}\end{array}$ & $\begin{array}{ll}0 & 0 \\
B_{2} & B_{2} \\
B_{3} & B_{3}\end{array}$ & $\begin{array}{l}\text { Jump to memory address } B_{3} \ldots B_{3} B_{2} \ldots B_{2} \text { if the condition } \\
\text { flip-flop } c \text { is false. Otherwise, execute the next instruction in sequence. }\end{array}$ \\
\hline JTC & (9 or 11$)$ & 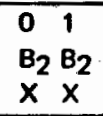 & 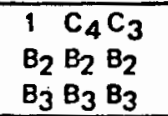 & $\begin{array}{l}0 \\
B_{2} \\
B_{3}\end{array}$ & 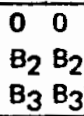 & $\begin{array}{l}\text { Jump to memory address } B_{3} \ldots B_{3} B_{2} \ldots B_{2} \text { if the condition } \\
\text { flip-flop } c \text { is true. Otherwise, execute the naxt instruction in sequence. }\end{array}$ \\
\hline CAL & (11) & $\begin{array}{l}01 \\
B_{2} B_{2} \\
\times \quad x\end{array}$ & $\begin{array}{lll} & x & x \\
B_{2} & B_{2} & B_{2} \\
B_{3} & B_{3} & B_{3}\end{array}$ & $\begin{array}{l} \\
B_{2} \\
B_{3}\end{array}$ & & $\begin{array}{l}\text { Unconditionally call the subroutine at memory address } B_{3} \ldots \\
B_{3} B_{2} \ldots B_{2} \text {. Save the current address (up one level in the stack). }\end{array}$ \\
\hline $\mathrm{CFe}$ & $(9$ or 11$)$ & $\begin{array}{l}01 \\
B_{2} B_{2} \\
\times \quad \times \\
\end{array}$ & $\begin{array}{lll}0 & C_{4} & C_{3} \\
B_{2} & B_{2} & B_{2} \\
B_{3} & B_{3} & B_{3}\end{array}$ & $\begin{array}{l} \\
B_{2} \\
B_{3}\end{array}$ & $\begin{array}{ll}1 & 0 \\
B_{2} & B_{2} \\
B_{3} & B_{3} \\
\end{array}$ & $\begin{array}{l}\text { Call the subroutine at memory address } B_{3} \ldots B_{3} B_{2} \ldots B_{2} \text { if the } \\
\text { condition flip.flop } c \text { is false, and save the current address lup one } \\
\text { level in the stack.l Otherwise, execute the next instruction in sequence. }\end{array}$ \\
\hline CTC & $(9$ or 11$)$ & $\begin{array}{l}01 \\
B_{2} B_{2} \\
\times \quad \times\end{array}$ & $\begin{array}{lll}1 & C_{4} & C_{3} \\
B_{2} & B_{2} & B_{2} \\
B_{3} & B_{3} & B_{3}\end{array}$ & $\begin{array}{l}\mathrm{O} \\
\mathrm{B}_{2} \\
\mathrm{~B}_{3}\end{array}$ & $\begin{array}{ll} & 0 \\
B_{2} & B_{2} \\
B_{3} & B_{3}\end{array}$ & $\begin{array}{l}\text { Call the subroutine at memory address } B_{3} \ldots B_{3} B_{2} \ldots B_{2} \text { if the } \\
\text { condition flip-flop } c \text { is true, and save the current address (up one } \\
\text { level in the stack). Otherwise, execute the next instruction in sequence. }\end{array}$ \\
\hline RET & (5) & 00 & $\times \times x$ & 1 & 11 & Unconditionally return (down one level in the stack). \\
\hline RFc & $\begin{array}{l}(3 \text { or } 5) \\
\end{array}$ & 00 & $0 c_{4} c_{3}$ & 0 & $\begin{array}{ll}1 & 1\end{array}$ & $\begin{array}{l}\text { Return (down one level in the stack) if the condition-flip-flop } c \text { is } \\
\text { false. Otherwise, execute the next instruction in sequence. }\end{array}$ \\
\hline RTC & $\mid 3$ or $5 \mid$ & 00 & $1 c_{4} c_{3}$ & $\mathbf{0}$ & $\begin{array}{ll}1 & 1\end{array}$ & $\begin{array}{l}\text { Return (down one level in the stack) if the condition flip-flop } c \text { is } \\
\text { true. Otherwise, execute the next instruction in sequence. }\end{array}$ \\
\hline RST & (5) & 00 & $A A A$ & 18 & 01 & Call the subroutine at memory address AAAOOO lup one level in the, st \\
\hline
\end{tabular}

Input/Output Instructions

\begin{tabular}{|l|l|lllll|l|}
\hline INP & $(8)$ & 0 & 1 & 0 & 0 & $M$ & $M$ \\
\hline OUT & $(6)$ & 0 & 1 & A R M & $\begin{array}{l}\text { Read the content of the selected input port (MMM) into the } \\
\text { accumulator. }\end{array}$ \\
\hline
\end{tabular}

\section{Machine Instruction}

\begin{tabular}{|c|c|ccccccccc|c|}
\hline HLT & $(4)$ & 0 & 0 & 0 & 0 & 0 & 0 & 0 & $\times$ & Enter the STOPPED state and remain there until interrupted. \\
\hline HLT & $(4)$ & 1 & 1 & 1 & 1 & 1 & 1 & 1 & 1 & Enter the STOPPED state and remain there until interrupted. \\
\hline
\end{tabular}

(1) SSS = Source Index Register 7 These registers, $r_{1}$, are designated A(accumulator-000),

DDD = Destination Index Register $\int \mathrm{B}(001), \mathrm{C}(010), \mathrm{D}(011), \mathrm{E}(100), \mathrm{H}(101), \mathrm{L}(110)$.

(2) Memory registers are addressed by the contents of registers $H \& L$.

(3) Additional bytes of instruction are designated by BBBBBBBB.

(4) $X=$ "Don't Care".

(5) Flag flip-flops are defined by $\mathrm{C}_{4} \mathrm{C}_{3}$ : carry (00-overflow or underflow), zero (01-result is zero), sign (10-MSB of result is "1"7. parity (11-parity is oven). 
1

\section{Complete Functional Definition}

The following pages present a detailed description of the complete 8008 Instruction Set.

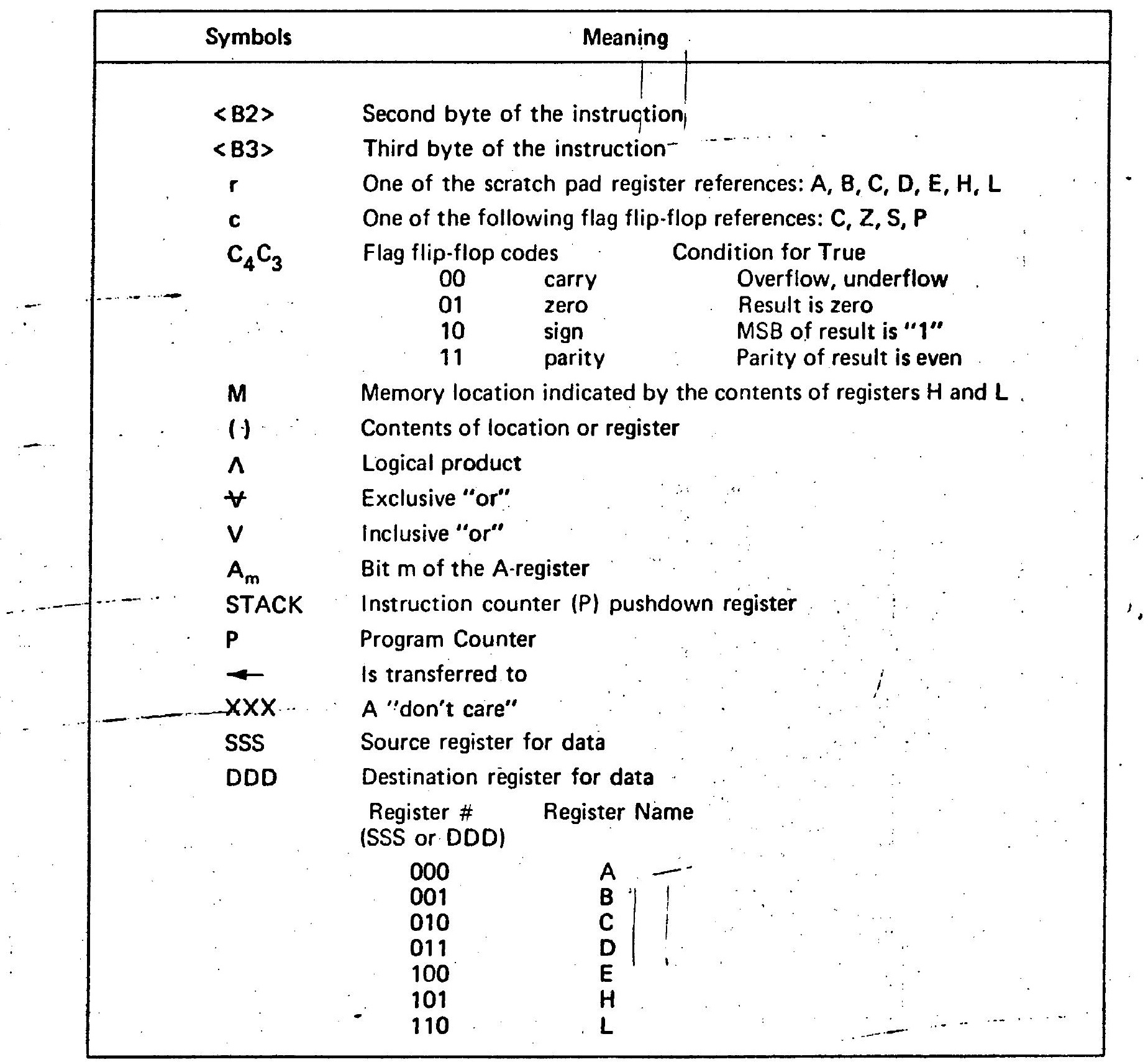




\section{INDEX REGISTER INSTRUCTIONS}

LOAD DATA TO INDEX REGISTERS - One Byte

Data may be loaded into or moved between any of the index registers, or memory registers.

\begin{tabular}{|c|c|c|c|c|}
\hline $\begin{array}{l}\mathrm{Lr} r_{1} r_{2} \\
\text { (one cycle - PCI) }\end{array}$ & 11 & DDD & SSS & $\begin{array}{l}\left(r_{1}\right)-\left(r_{2}\right) \text { Load register } r_{1} \text { with the content of } r_{2} \text {. } \\
\text { The content of } r_{2} \text { remains unchanged. If SSS=DDD, } \\
\text { the instruction is a NOP (no operation). }\end{array}$ \\
\hline $\begin{array}{l}\text { LrM } \\
\text { (two cycles - } \\
\text { PCI/PCR) }\end{array}$ & 11 & DDD & 111 & $\begin{array}{l}(r)-(M) \text { Load register } r \text { with the content of the } \\
\text { memory location addressed by the contents of } \\
\text { registers } H \text { and } L \text {. (DDD } \neq 111-H A L T \text { instr.) }\end{array}$ \\
\hline $\begin{array}{l}\mathrm{LMr} \\
\text { (two cycles - } \\
\mathrm{PCl} / \mathrm{PCW} \text { ) }\end{array}$ & 11 & 111 & SSS & $\begin{array}{l}(M)-(r) \text { Load the memory location addressed by } \\
\text { the contents of registers } H \text { and } L \text { with the content } \\
\text { of register } r . \text { (SSS } \neq 111-H A L T \text { instr.) }\end{array}$ \\
\hline
\end{tabular}

LOAD DATA IMMEDIATE - Two Bytes

A byte of data immediately following the instruction may be loaded into the processor or into the memory

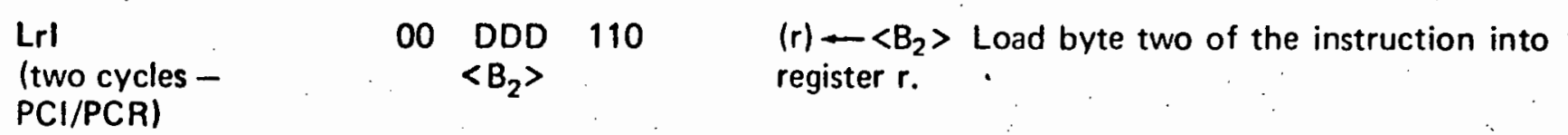

$\begin{array}{lll}\text { LMI } & 00 \quad 111 & 110 \\ \text { (three cycles } & \left\langle B_{2}\right\rangle\end{array}$

(M) $-\left\langle\mathrm{B}_{2}\right\rangle$ Load byte two of the instruction into the memory location addressed by the contents of registers $H$ and $L$.
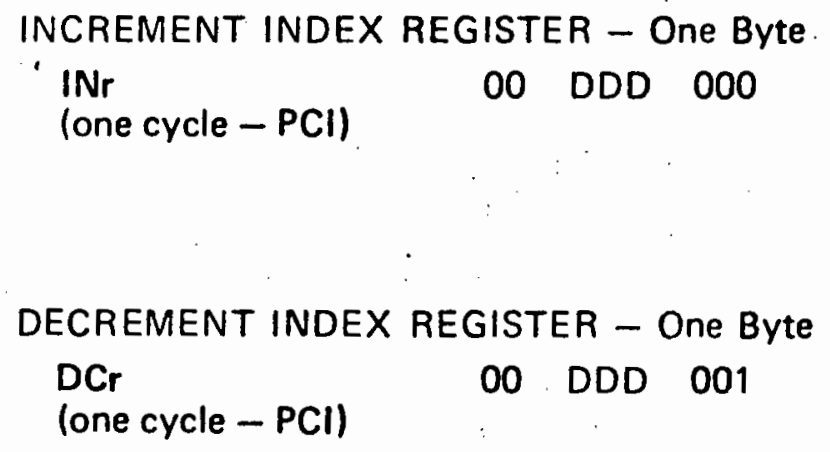

$(r)-(r)+1$. The content of register $r$ is incremented by one. All of the condition flip-flops except carry are affected by the result. Note that DDD $\neq 000$ (HALT instr.) and DDD $\neq 111$ (content of memory may not be incremented).

$(r)-(r)-1$. The content of register $r$ is decremented by one. All of the condition flip-flops except carry are affected by the result. Note that DDD $\neq 000$ (HALT instr.) and $D D D \neq 111$ (content of memory may not be decremented).

\section{ACCUMULATOR GROUP INSTRUCTIONS}

Operations are performed and the status flip-flops, $C, Z, S, P$, are set based on the result of the operation. Logical operations (NDr, XRr, ORr) set the carry flip-flop to zero. Rotate operations affect only-the carry flip-flop. Two's complement subtraction is used.

\section{ALU INDEX REGISTER INSTRUCTIONS - One Byte}

(one cycle - PCI)

Index Register operations are carried out between the accumulator and the content of one of the index registers (SSS=000 thru SSS=110). The previous content of register SSS is unchanged by the operation.

\begin{tabular}{|c|c|c|c|c|c|}
\hline ADr & & 10 & 000 & SSS & $\begin{array}{l}(A)-(A)+(r) \text { Add the content of register } r \text { to the } \\
\text { content of register } A \text { and place the result into } \\
\text { register } A \text {. }\end{array}$ \\
\hline $\mathrm{ACr}$ & 1 & 10 & 001 & SSS & $\begin{array}{l}(A)-(A)+(r)+(\text { carry) Add the content of register } r \\
\text { and the contents of the carry flip-flop to the content } \\
\text { of the } A \text { register and place the result into Register } A \text {. }\end{array}$ \\
\hline SUr & & 10 & 010 & SSS & $\begin{array}{l}(A)-(A)-(r) \text { Subtract the content of register } r \text { from } \\
\text { the content of register } A \text { and place the result into } \\
\text { register } A \text {. Two's complement subtraction is used. }\end{array}$ \\
\hline
\end{tabular}


ACCUMULATOR GROUP INSTRUCTIONS - Cont'd.

$\begin{array}{lrrrr}\text { SBr } & \ldots & 011 & \text { SSS } \\ & & 10 & 100 & \text { SSS } \\ \text { NDr } & 10 & 101 & \text { SSS } \\ \text { XRr } & 10 & 110 & \text { SSS } \\ \text { ORr } & 10 & 111 & \text { SSS } \\ \text { CPr } & & 11 & & \\ \end{array}$

\section{ALU OPERATIONS WITH MEMORY - One Byte}

(two cycles - PCI/PCR)

Arithmetic and logical operations are carried out between the accumulator and the byte of data addressed by the contents of registers $H$ and $L$.

$\begin{array}{lllll}\text { ADM } & 10 & 000 & 111 & (A)-(A)+(M) \text { ADD } \\ \text { ACM } & 10 & 001 & 111 & (A)-(A)+(M)+(\text { carry) ADD with carry } \\ \text { SUM } & 10 & 010 & 111 & (A)-(A)-(M) \text { SUBTRACT } \\ \text { SBM } & 10 & 011 & 111 & (A)-(A)-(M)-(\text { borrow) SUBTRACT with borrow } \\ \text { NDM } & 10 & 100 & 111 & (A)-(A) \wedge(M) \text { Logical AND } \\ \text { XRM } & 10 & 101 & 111 & (A)-(A) \forall(M) \text { Exclusive OR } \\ \text { ORM } & 10 & 110 & 111 & (A)-(A) V(M) \text { Inclusive OR } \\ \text { CPM } & 10 & 111 & 111 & \text { (A)-(M) COMPARE }\end{array}$

\section{ALU IMMEDIATE INSTRUCTIONS - Two Bytes}

(two cycles -PCI/PCR)

Arithmetic and logical operations are carried out between the accumulator and the byte of data immediately following the instruction.

\begin{tabular}{|c|c|c|c|c|}
\hline$A D I$ & 00 & $\begin{array}{l}000 \\
\left\langle B_{2}\right\rangle\end{array}$ & 100 & $\begin{array}{l}(A)-(A)+\left\langle B_{2}>\right. \\
A D D\end{array}$ \\
\hline $\mathrm{ACl}$ & 00 & $\begin{array}{l}001 \\
\left\langle B_{2}\right\rangle\end{array}$ & 100 & $\begin{array}{l}(A)--(A)+\left\langle B_{2}\right\rangle+(\text { carry }) \\
A D D \text { with carry }\end{array}$ \\
\hline SUI & 00 & $\begin{array}{l}010 \\
\left\langle\mathrm{~B}_{2}\right\rangle\end{array}$ & 100 & $\begin{array}{l}(A)-(A)-\left\langle B_{2}\right\rangle \\
\text { SUBTRACT }\end{array}$ \\
\hline SBI & 00 & $\begin{array}{l}011 \\
\left\langle B_{2}\right\rangle\end{array}$ & 100 & $\begin{array}{l}\text { (A) }-(A)-<B_{2}>\text {-(borrow) } \\
\text { SUBTRACT with borrow }\end{array}$ \\
\hline NDI & 00 & $\begin{array}{l}100 \\
\left\langle B_{2}\right\rangle\end{array}$ & 100 & $\begin{array}{l}(A)-(A) \wedge\left\langle B_{2}>\right. \\
\text { Logical AND }\end{array}$ \\
\hline XRI & 00 & $\begin{array}{l}101 \\
\left\langle B_{2}\right\rangle\end{array}$ & 100 & $\begin{array}{l}(A)-(A) \forall\left\langle B_{2}\right\rangle \\
\text { Exclusive OR }\end{array}$ \\
\hline ORI & 00 & $\begin{array}{c}110 \\
\left\langle B_{2}\right\rangle\end{array}$ & 100 & $\begin{array}{l}(A)-(A) \vee<B_{2}> \\
\text { Inclusive } O R\end{array}$ \\
\hline CPI & 00 & $\begin{array}{l}111 \\
\left\langle B_{2}\right\rangle\end{array}$ & 100 & $\begin{array}{l}(A)-\left\langle B_{2}\right\rangle \\
\text { COMPARE }\end{array}$ \\
\hline
\end{tabular}




\section{ROTATE INSTRUCTIONS - One Byte \\ (one cycle - PCI)}

The accumulator content (register A) may be rotated either right or left, around the carry bit or through the carry bit. Only the carry flip-flop is affected by these instructions; the other flags are unchanged.
RLC
00
010
$A_{m+1}-A_{m}, A_{0}-A_{7}$, (carry) $-A_{7}$

Rotate the content of register $A$ left one bit.

RRC

$00 \quad 001 \quad 010$

Rotate $A_{7}$ into $A_{0}$ and into the carry flip-flop.

$A_{m}-A_{m+1}, A_{7}-A_{0}$, (carry) $-A_{0}$

Rotate the content of register $A$ right one bit.

Rotate $A_{0}$ into $A_{7}$ and into the carry flip-flop.

RAL

$00 \quad 010 \quad 010$

$A_{m+1}-A_{m}, A_{0}-$ (carry), (carry) $-A_{7}$

Rotate the content of Register $A$ left one bit.

Rotate the content of the carry flip-flop into $A_{0}$.

Rotate $A_{7}$ into the carry flip-flop.

RAR

$00 \quad 011 \quad 010$

$A_{m}-A_{m+1}, A_{7}-$ (carry), (carry) $-A_{0}$

Rotate the content of register $A$ right one bit.

Rotate the content of the carry flip-flop into $A_{7}$.

Rotate $A_{0}$ into the carry flip-flop.

\section{PROGRAM COUNTER AND STACK CONTROL INSTRUCTIONS}

JUMP INSTRUCTIONS - Three Bytes

(three cycles - PCI/PCR/PCR)

Normal flow of the microprogram may be altered by jumping to an address specified by bytes two and three of an instruction.

\begin{tabular}{|c|c|c|}
\hline $\begin{array}{l}\text { JMP } \\
\text { (Jump Uncondition }\end{array}$ & 01 & $\begin{array}{r}X X X \\
\left\langle\left\langle B_{2}\right\rangle\right. \\
\left\langle B_{3}\right\rangle\end{array}$ \\
\hline $\begin{array}{l}\text { JFc } \\
\text { (Jump if Condition } \\
\text { False) }\end{array}$ & 01 & $\begin{array}{l}\mathrm{OC}_{4} \mathrm{C}_{3} \\
\left\langle\mathrm{~B}_{2}\right\rangle \\
\left\langle\mathrm{B}_{3}\right\rangle\end{array}$ \\
\hline $\begin{array}{l}\text { JTc } \\
\text { (Jump if Condition } \\
\text { True) }\end{array}$ & 01 & $\begin{array}{l}1 C_{4} C_{3} \\
\left\langle B_{2}\right\rangle \\
\left\langle B_{3}\right\rangle\end{array}$ \\
\hline
\end{tabular}

(P) $-\left\langle B_{3}\right\rangle\left\langle B_{2}\right\rangle$ Jump unconditionally to the instruction located in memory location addressed by byte two and byte three.

If $(c)=0,(P)-\left\langle B_{3}\right\rangle\left\langle B_{2}\right\rangle$. Otherwise, $(P)=(P)+3$. If the content of flip-flop $c$ is zero, then jump to the instruction located in memory location $\left\langle\mathrm{B}_{3}\right\rangle\left\langle\mathrm{B}_{2}\right\rangle$; otherwise, execute the next instruction in sequence.

If $(c)=1,(P)-\left\langle B_{3}\right\rangle\left\langle B_{2}\right\rangle$. Otherwise, $(P)=(P)+3$. If the content of flip-flop $c$ is one, then jump to the instruction located in memory location $\left\langle\mathrm{B}_{3}\right\rangle\left\langle\mathrm{B}_{2}\right\rangle$; otherwise, execute the next instruction in sequence.

CALL INSTRUCTIONS - Three Bytes

(three cycles - PCl/PCR/PCR)

Subroutines may be called and nested up to seven levels.

\begin{tabular}{|c|c|c|}
\hline $\begin{array}{l}\text { CAL } \\
\text { (Call subroutine } \\
\text { Unconditionally) }\end{array}$ & 01 & $\begin{array}{l}X X X \\
\left\langle B_{2}\right\rangle \\
\left\langle B_{3}\right\rangle\end{array}$ \\
\hline $\begin{array}{l}\text { CFc } \\
\text { (Call subroutine } \\
\text { if Condition False) }\end{array}$ & 01 & $\begin{array}{l}\mathrm{OC}_{4} \mathrm{C}_{3} \\
\left\langle\mathrm{~B}_{2}\right\rangle \\
\left\langle\mathrm{B}_{3}\right\rangle\end{array}$ \\
\hline
\end{tabular}

(Stack) $-(P),(P)-\left\langle B_{3}\right\rangle\left\langle B_{2}\right\rangle$. Shift the content of $P$. to the pushdown stack. Jump unconditionally to the instruction located in memory location addressed by byte two and byte three.

If $(c)=0$. (Stack $)-(P), \quad(P)-\left\langle B_{3}\right\rangle\left\langle B_{2}\right\rangle$. Otherwise, $(P)=(P)+3$. If the content of flip-flop $c$ is zero, then shift contents of $P$ to the pushdown stack and jump to the instruction located in memory location $\left\langle B_{3}\right\rangle\left\langle B_{2}\right\rangle$; otherwise, execute the next instruction in sequence.

$\begin{array}{lll}\text { CTc } & 01 & 1 C_{4} C_{3} \quad 010 \\ & \left\langle B_{2}\right\rangle \\ \text { (Call subroutine } & \left\langle B_{3}\right\rangle \\ \text { if Condition True) } & \left\langle B_{3}\right\rangle\end{array}$

If $(c)=1,($ Stack $)-(P),(P)-\left\langle B_{3}\right\rangle\left\langle B_{2}\right\rangle$. Otherwise, $(P)=(P)+3$. If the content of flip flop $c$ is one, then shift contents of $P$ to the pushdown stack and jump to the instruction located in memory location $\left\langle B_{3}\right\rangle\left\langle B_{2}\right\rangle$; otherwise, execute the next instruction in sequence.

In the above JUMP and CALL instructions $\left\langle B_{2}\right\rangle$ contains the least significant half of the address and $\left\langle B_{3}\right\rangle$ contains the most significant half of the address. Note that $D_{6}$ and $D_{7}$ of $\left\langle B_{3}>\right.$ are "don't care" bits since the CPU uses fourteen bits of address. 


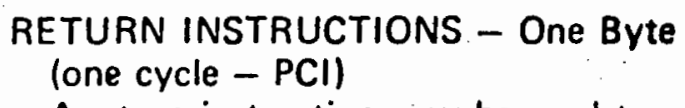

A return instruction may be used to exit from a subroutine; the stack is popped-up one level at a time. RET

$00 \times \times \times 111$ (P)-(Stack). Return to the instruction in the memory location addressed by the last value shifted into the pushdown stack. The stack pops up one level.

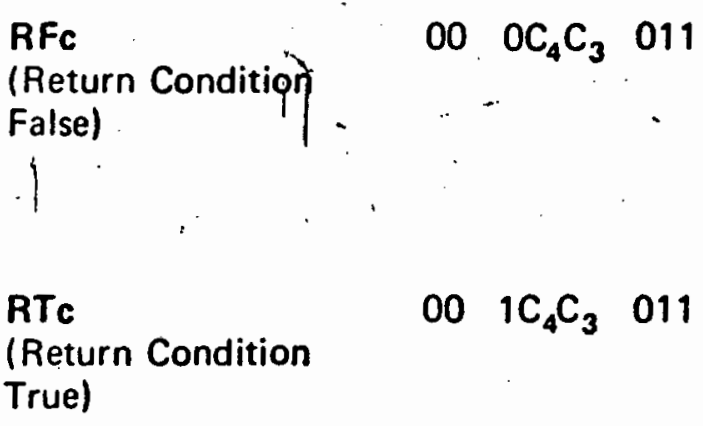

If $(c)=0,(P)-($ Stack $)$; otherwise, $(P)=(P)+1$.

If the content of flip-flop $c$ is zero, then return to the instruction in the memory location addressed by the last value inserted in the pushdown stack. The stack pops up one level. Otherwise, execute the next instruction in sequence.

(Return Condition

If $(c)=1,(P)-($ Stack $)$; otherwise, $(P)=(P)+1$.

If the content of flip-flop $c$ is one, then return to the instruction in the memory location addressed by the last value inserted in the pushdown stack. The stack pops up one level. Otherwise, execute the next instruction

RESTART INSTRUCTION - One BYte in sequence.

(one cycle - PCI)

The restart instruction acts as a one byte call on eight specified locations of page 0 , the first 256 instruction words.
RST
00 AAA 101

(Stack)-(P),(P)-1000000 00AAA000)

Shift the contents of $P$ to the pushdown stack.

The content, AAA, of the instruction register is shifted into bits 3 through 5 of the P-counter. All other bits of the P-counter are set to zero. As a oneword "call", eight eight-byte subroutines may be accessed in the lower 64 words of memory.

\section{One Byte \\ (two cycles - PCI/PCC)}

INPUT/OUTPUT INSTRUCTIONS

Eight input devices may be referenced by the input instruction

INP $\quad 0100 M$ MM1 $\quad$ (A)-(input data lines). The content of register A is made available to external equipment at state $T 1$ of the PCC cycle. The content of the instruction register is made available to external equipment at state T2 of the PCC cycle. New data for the accumulator is loaded at T3 of the PCC cycle. MMM denotes input device number. The content of the condition flip-flops, $S, Z, P, C$, is output on $D_{0}, D_{1}, D_{2}, D_{3}$ respectively at $\mathrm{T} 4$ on the $\mathrm{PCC}$ cycle.

Twenty-four output devices may be referenced by the output instruction.
OUT
01 RRM MMI
(Output data lines)-(A). The content of register $A$ is made available to external equipment at state $\mathrm{T} 1$ and the content of the instruction register is made available to external equipment at state T2 of the PCC cycle. RAMMM denotes output device number $(R R \neq$ 001 .

HALT INSTRUCTION - One Byte (one cycle - PCI)

$\begin{array}{cccc}\text { HLT } & 000 & 00 \times \\ & \text { or } & \\ 11 & 111 & 111\end{array}$

\section{MACHINE INSTRUCTION}

On receipt of the Halt Instruction, the activity of the processor is immediately suspended in the STOPPED state. The content of all registers and memory is unchanged. The P-counter has been updated and the internal dynamic memories continue to be refreshed. 


\section{Internal Processor Operation}

Internally the processor operates through five different states:

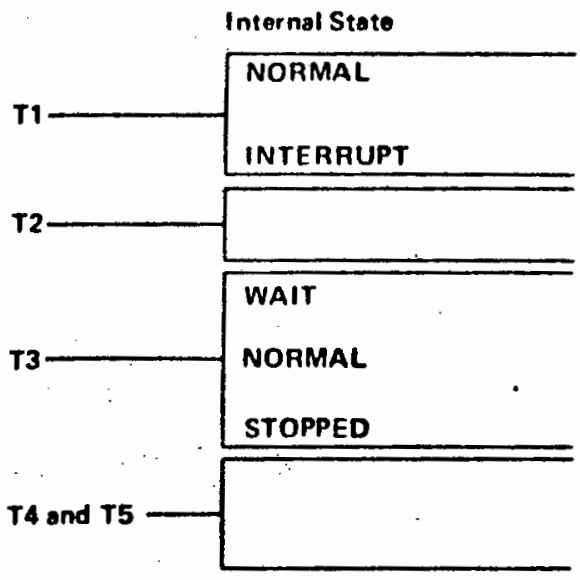

Trpical function

Send out lower eight bits of address and increment program counter.

Send out lower eight bits of address and suppress incrementing of program counter and acknowledge interrupt.

Send out six higher order bits of address and two control bits, $D_{6}$ and $D_{7}$. Increment program counter if there has been a carry from T1.

Wait for READY signal to come true. Refresh internal dynamic memories while waiting.

Fetch and decode instruction; fetch data from memory; output data to memory. Refresh internal memories.

Remain stopped until INTERRUPT occurs. Refresh internal memories.

Execute instruction and appropriately transfer data within processor. Content of data bus transfer is available at $1 / 0$ bus for convenience in testing. Some cycles do not require these states. In those cases, the states are skipped and the processor goes directly to T1.

The 8008 is driven by two non-overlapping clocks. Two clock periods are required for each state of the processor. $\phi_{1}$ is generally used to precharge all data lines and memories and $\phi_{2}$ controls all data transfers within the processor. A SYNC signal (divide by two of $\phi_{2}$ ) is sent out by the 8008 . This signal distinguishes between the two clock periods of each state.

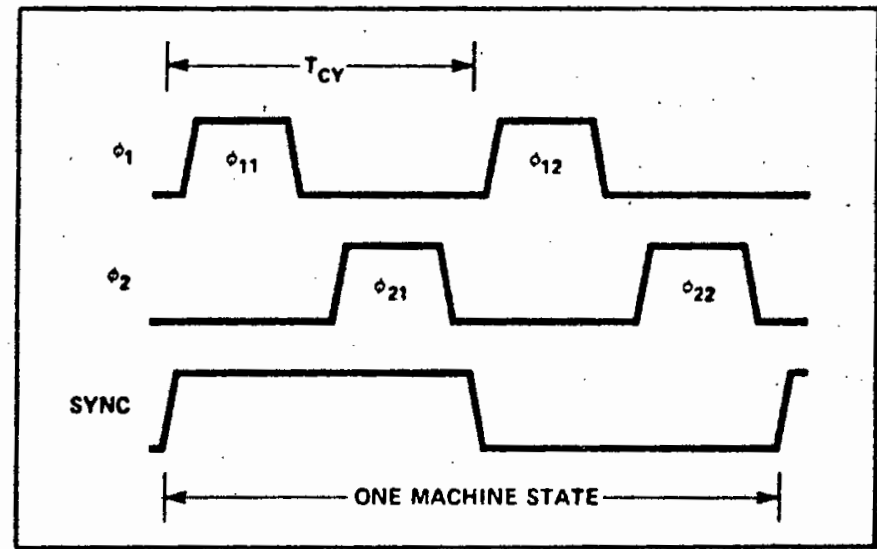

Processor Clocks

The figure below shows state transitions relative to the internal operation of the processor. As noted in the previous table, the processor skips unnecessary execution steps during any cycle. The state counter within the 8008 operates is a five bit feedback shift register with the feedback path controlled by the instruction being executed. When the processor is either waiting or stopped, it is internally cycling through the T3 state. This state is the only time in the cycle when the internal dynamic memories can be refreshed.

ICYCLE 1) (HLT + INT + RETURN (CF) + (CYCLE 2) (OUT + LMH) + (CYCLE 3) (LMI + JUMP (CF) + CALL (CF)

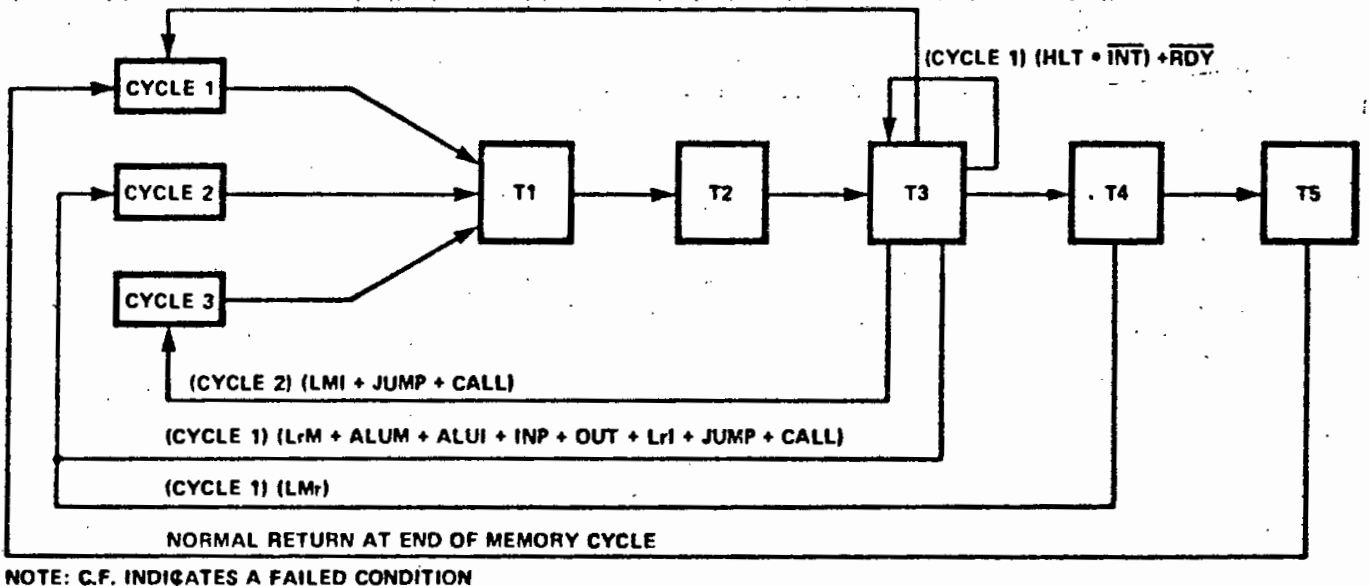

Transition State Diagram (Internal)

The following pages show the processor activity during each state of the execution of each instruction. 
INTERNAL PROCESSOR OPERATION

INDEX REGISTER INSTRUCTIONS

\begin{tabular}{|c|c|c|c|c|c|c|c|c|c|}
\hline \multicolumn{3}{|c|}{ INSTRUCTION CODING } & \multirow{2}{*}{ OPERATION } & \multirow{2}{*}{$\begin{array}{l}\text { \#OF STATES } \\
\text { TO EXECUTE } \\
\text { INSTRUCTION }\end{array}$} & \multicolumn{5}{|c|}{ MEMORY CYCLE ONE (I) } \\
\hline $\mathrm{D}_{7} \mathrm{D}_{6}$ & $D_{5} D_{4} D_{3}$ & $D_{2} D_{1} D_{0}$ & & & $T 1(2)$ & T2 & 73 & $T 4(3)$ & T5 \\
\hline 11 & D D D & 5 s s & $\operatorname{Lr}_{1} r_{2}$ & (5) & $\begin{array}{r}P C_{L} \text { OUT } \\
(4) \\
\end{array}$ & $\mathrm{PC}_{\mathrm{H}} \mathrm{OUT}$ & $\begin{array}{l}\text { FETCH INSTA. (5) } \\
\text { TO IR \& REG. }\end{array}$ & $\begin{array}{c}\text { SSS TO REG. b } \\
\text { (6) }\end{array}$ & REG. b TO DDD \\
\hline 11 & DDD & 111 & LrM & (8) & PGLOUT & $\mathrm{PC}_{\mathrm{H}} \mathrm{OUT}$ & $\begin{array}{l}\text { FETCH INSTR. } \\
\text { TO IR \& REG. }\end{array}$ & (7) & \\
\hline 11 & $\begin{array}{lll}1 & 1 & 1\end{array}$ & s s s & LMr & (7) & $P C_{L}$ OUT & PCHOUT & $\begin{array}{l}\text { FETCH INSTR. } \\
\text { TOIR \& REG.b }\end{array}$ & SSS TO REG. $b$ & - \\
\hline 00 & DOD & 710 & Lil & (8) & PCLOUT & PCHOUT & $\begin{array}{l}\text { FETCH INSTR. } \\
\text { TO IR \& REG. }\end{array}$ & & \\
\hline 00 & 111 & 110 & LMI & (9) & $P C_{L}$ OUT & $P C_{H} O U T$ & $\begin{array}{l}\text { FETCH INSTR. } \\
\text { TO IR \& REG. } b\end{array}$ & & \\
\hline 00 & DDD & 000 & $\mathrm{INr}$ & (5) & PCLOUT & $P C_{H}$ OUT & $\begin{array}{l}\text { FETCH INSTR. } \\
\text { TO IR \& REG. b }\end{array}$ & $\bar{x}$ & $\begin{array}{l}\text { ADD OP - FLAGS } \\
\text { AFFECTED }\end{array}$ \\
\hline 00 & DOD & 001 & $\begin{array}{r}\mathrm{DCr} \\
-\cdots\end{array}$ & (5) & $P C_{L}$ OUT & $\mathrm{PC}_{\mathrm{H}} \mathrm{OUT}$ & $\begin{array}{l}\text { FETCH INSTR. } \\
\text { TO IR \& REG. }\end{array}$ & $\bar{x}$ & $\begin{array}{l}\text { SUB OP - FLAGS } \\
\text { AFFECTED }\end{array}$ \\
\hline
\end{tabular}

\begin{tabular}{|c|c|c|c|c|c|c|c|c|c|c|}
\hline 1 & 0 & $P P P$ & S S s & ALUOP & (5) & PCLOUT & $\mathrm{PC}_{\mathrm{H}} \mathrm{OUT}$ & $\begin{array}{l}\text { FETCH INSTR. } \\
\text { TO IR \& REG. }\end{array}$ & SSS TO REG, $b$ & $\begin{array}{l}\text { ALU OP - FLAGS } \\
\text { AFFECTED }\end{array}$ \\
\hline 1 & & $P P P$ & 111 & ALU OPM & (8) & PCLOUT & $\mathrm{PC}_{\mathrm{H}} \mathrm{OUT}$ & $\begin{array}{l}\text { FETCH INSTR. } \\
\text { TO IR \& REG. b }\end{array}$ & & \\
\hline 0 & 0 & $P P P$ & 100 & ALU OPI & (8) & $P_{\text {PCOUT }}$ & PCHOUT & $\begin{array}{l}\text { FETCH INSTR. } \\
\text { TOIR \& AEG.b }\end{array}$ & & \\
\hline & 0 & $\begin{array}{lll}0 & 0 & 0 \\
- & - & \end{array}$ & 010 & RLC & $|5|$ & PCLOUT & PCHOUT & $\begin{array}{l}\text { FETCH INSTR. } \\
\text { TO IR \& REG. }\end{array}$ & $\bar{x}$ & $\begin{array}{l}\text { ROTATE REG. A } \\
\text { CARAY AFFECTED }\end{array}$ \\
\hline 00 & & $\begin{array}{lll}0 & 0 & 1\end{array}$ & 010 & RRC & (5) & $\mathrm{PC}_{\mathrm{L}} \mathrm{OUT}$ & $\mathrm{PC}_{\mathrm{HOUT}}$ & $\begin{array}{l}\text { FETCH INSTR. } \\
\text { TOIR \& REG. }\end{array}$ & $\bar{x}$ & $\begin{array}{l}\text { ROTATE REG. A } \\
\text { CARRY AFFECTEO }\end{array}$ \\
\hline$\overline{\mathrm{u}} \mathrm{c}$ & & 010 & 010 & RAL & (5) & $P C_{L} O U T$ & PCHOUT & $\begin{array}{l}\text { FETCH INSTR. } \\
\text { TOIR \& AEG. }\end{array}$ & $\bar{x}$ & $\begin{array}{l}\text { ROTATE REG. A } \\
\text { CARRY AFFECTED }\end{array}$ \\
\hline 00 & 0 & $\begin{array}{lll}0 & 1 & 1\end{array}$ & 010 & RAR & (5) & $P C_{L}$ OUT & $\mathrm{PC}_{\mathrm{HOUT}}$ & $\begin{array}{l}\text { FETCH INSTA. } \\
\text { TO IA \& REG. } b\end{array}$ & $\bar{x}$ & $\begin{array}{l}\text { ROTATE REG. A } \\
\text { CARRY AFFECTED }\end{array}$ \\
\hline
\end{tabular}

PROGRAM COUNTER AND STACK CONTROL INSTRUCTIONS

\begin{tabular}{|c|c|c|c|c|c|c|c|c|c|}
\hline 01 & $x \times x$ & 10 & JMP & (11) & PCLOUT & $\mathrm{PC}_{\mathrm{H}} \mathrm{OUT}$ & $\begin{array}{l}\text { FETCH INSTR. } \\
\text { TO IR \& REG. }\end{array}$ & & \\
\hline 01 & $u c c$ & 000 & $\mathrm{JFc}$ & $(9$ or 111$)$ & PCLOUT & $\mathrm{PC}_{\mathrm{H}} \mathrm{OUT}$ & $\begin{array}{l}\text { FETCH INSTR. } \\
\text { TO IR \& REG. }\end{array}$ & & \\
\hline 01 & 1 C C & 00 & JTc & 19 or 111 & $P C_{L}$ OUT & $\overline{P C_{H O U T}}$ & $\begin{array}{l}\text { FETCH INSTR. } \\
\text { TO IR \& REG. b }\end{array}$ & & \\
\hline 01 & $x \times x$ & 110 & $\overline{\text { CAL }}$ & (11) & PCLOUT & $\overline{P C}_{H O U T}$ & $\begin{array}{l}\text { FETCH INSTR, } \\
\text { TO IR \& REG. }\end{array}$ & & \\
\hline $0 !$ & $\therefore \mathrm{CC}$ & 010 & CFc & (9 or 11$)$ & $P C_{L}$ OUT & $\mathrm{PC}_{\mathrm{H}} \mathrm{OUT}$ & $\begin{array}{l}\text { FETCHINSTA. } \\
\text { TO IR \& REG. }\end{array}$ & & \\
\hline $0 \cdot 1$ & $1 \cdot C \mathrm{C}$ & 010 & CTC & (9 or 11$)$ & $P C_{L}$ OUT & PCHOUT & $\begin{array}{l}\text { FETCH INSTA. } \\
\text { TOIR \& REG. b }\end{array}$ & & \\
\hline 00 & $x \times x$ & 111 & RET & (5) & $\overline{P C_{L} \text { OUT }}$ & PCHOUT & $\begin{array}{l}\text { FETCH INSTR. } \\
\text { TO IR \& REG. }\end{array}$ & POP STACK & $\bar{x}$ \\
\hline 00 & $0 \mathrm{CC}$ & 011 & Af & 13 or 51 & $P C_{L} O U T$ & $\mathrm{PC} \mathrm{C}_{\mathrm{H}} \mathrm{OUT}$ & $\begin{array}{l}\text { FETCH INSTR. } \\
\text { TOIR \& REG. b }\end{array}$ & POP STACK (13) & $\bar{x}$ \\
\hline 00 & $1 \mathrm{cc}$ & 011 & RTc & $(3$ or 5$)$ & PCLOUT & PCHOUT & $\begin{array}{l}\text { FETCH INSTR. } \\
\text { TO IP \& REG. }\end{array}$ & POP STACK (13) & $x$ \\
\hline & $A A A$ & 101 & $\begin{array}{c}\text { RST } \\
\cdots \\
-\end{array}$ & (5) & PCLOUT. & $\mathrm{PC}_{\mathrm{H}} \mathrm{OUT}$ & $\begin{array}{l}\text { FETCH INSTR. } \\
\text { TOREG.b AND } \\
\text { PUSH STACK } \\
\text { (O-REG. al }\end{array}$ & REG. a $T O P C_{H}$ & $\begin{array}{c}\text { REG. D TO PC } \\
\text { (14) }\end{array}$ \\
\hline
\end{tabular}

$1 / 0$ INSTRUCTIONS

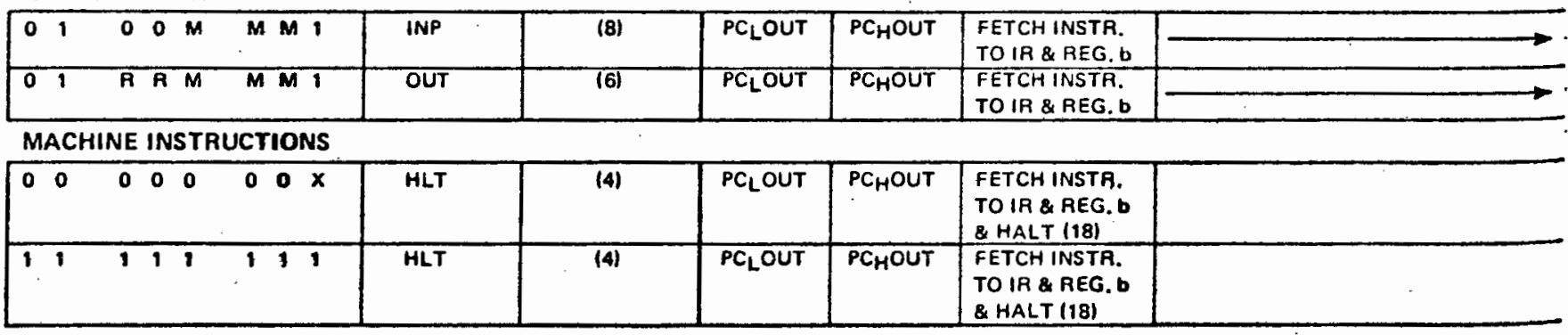

\section{NOTES:}

1. The first memory cyele is always a PCl (instruction) cyclo.

2. Internally, states ere defined es T1 through T5. It some cases

more than one memory cycle is required to execute an instruction.

3. Content of the internal date bus at T4 end T5 is aveilable at the

dats bus. This is designed for testing purposes only.

4. Lower order address bits in the program counter are denoted

by $P C_{L}$ and higher order bits are designated by $P C_{H}$.

5. During an instruction fetch the instruction comes from memory to the instruction register and is decoded.
6. Temporary registers are used internally for arithmetic operations and date transfers (Register a and Registar b.l

7. These states are skipped.

8. PCR cycle (Memory Read Cycte).

9: " $X$ " denotes an idle state.

10. PCW cycle (Mamory Writo Cycto).

11. When the JUMP is conditional and ithe condition falis, sutes T4 and T5 are skipped and the state counter advances to the next memary cycle. 


\begin{tabular}{|c|c|c|c|c|c|c|c|c|c|}
\hline \multicolumn{5}{|c|}{ MEMORY CYCLE TWO } & \multicolumn{5}{|c|}{ MEMORY CYCLE THREE } \\
\hline$r$ & 12 & $\mathbf{T 3}$ & $T 4$ & T5 & $T 1$ & T2 & $\mathbf{T 3}$ & TA & T5 \\
\hline $\begin{array}{l}\text { REG.L OUT } \\
\text { (B) }\end{array}$ & REG. H OUT & $\begin{array}{l}\text { DATA TO } \\
\text { REG. b } \\
\end{array}$ & $\begin{array}{l}x \\
(9) \\
\end{array}$ & $\begin{array}{l}\text { AEG. } \\
\text { TODOD }\end{array}$ & & & & & \\
\hline $\begin{array}{l}\text { REG. L OUT } \\
\text { (110) }\end{array}$ & REG. H OUT & \begin{tabular}{|l|} 
REG.'b \\
TO OUT \\
\end{tabular} & & & & & & & \\
\hline$P C_{L}$ OUT (8) & ${ }^{P C_{H} O U T}$ & $\begin{array}{l}\text { DATA TO } \\
\text { REG. } 6\end{array}$ & $x$ & $\begin{array}{l}\text { REG. b } \\
\text { TODDD }\end{array}$ & & & & & \\
\hline PCLOUT (8) & PCHOUT & $\begin{array}{l}\text { DATA TO } \\
\text { REG.b }\end{array}$ & & $\rightarrow$ & $\begin{array}{l}\text { REG.L } \\
\text { OUT(10) }\end{array}$ & $\begin{array}{c}\text { REG.H } \\
\text { OUT }\end{array}$ & $\begin{array}{l}\text { REG. } \\
\text { TO OUT } \\
\end{array}$ & & \\
\hline & & & & & & & & & \\
\hline & & & & & & & & & \\
\hline REG.L OUT & REG. HOUT & DATA TO & $x$ & ALUOP - FLAGS & & & & & \\
\hline$\frac{|8|}{P C_{L} O U T(B)}$ & PCHOUT & $\begin{array}{l}\text { REG.b } \\
\text { DATA TO } \\
\text { REG.b } \\
\end{array}$ & $x$ & $\begin{array}{l}\text { AFFECTED } \\
\text { AAITH OP - FLAGS } \\
\text { AFFECTED }\end{array}$ & & & & & \\
\hline & & & & & & & & & \\
\hline & & & & & & & & & \\
\hline & & & & . & & & & & \\
\hline & & . & & & & & & & \\
\hline & & LOWER ADD. & & & $P C, O U T(B)$ & $\mathrm{PC}_{\mathrm{H}} \mathrm{OUT}$ & HIGHER ADD, & REG. & REG.b \\
\hline PCLOUT(8) & $\mathrm{PC}_{\mathrm{H}} \mathrm{OUT}$ & TO REG. 6 & & $\rightarrow$ & $P C L O U 181$ & PCHOUT & AEG. a & TO $P C_{H}$ & TO PC \\
\hline$P C_{L}$ OUT (B) & $\mathrm{PC}_{\mathrm{H}} \mathrm{OUT}$ & $\begin{array}{l}\text { LOWER ADD. } \\
\text { TO REG. } \mathrm{b}\end{array}$ & & $\rightarrow$ & PCLOUT (8) & $\mathrm{PC}_{\mathrm{H}} \mathrm{OUT}$ & $\begin{array}{r}\text { HIGHER ADD. } \\
\text { REG. a (111) }\end{array}$ & $\begin{array}{l}\text { AEG. } \\
\text { TO PC }\end{array}$ & $\begin{array}{l}\text { REG.b } \\
\text { TO PC }\end{array}$ \\
\hline PG OUT (8) & $\mathrm{PC}_{\mathrm{H}} \mathrm{OUT}$ & $\begin{array}{l}\text { LOWER ADD. } \\
\text { TO AEG. } \mathrm{b}\end{array}$ & $\ldots$ & $\rightarrow$ & $P C_{L}$ OUT(8) & $\mathrm{PC}_{\mathrm{HOUT}}$ & $\begin{array}{r}\text { HIGHER ADD } \\
\text { PEG. a } 1111 \\
\end{array}$ & $\begin{array}{l}\text { REG. } \\
\text { TO } \text { PC }_{H}\end{array}$ & $\begin{array}{l}\text { REG.b } \\
\text { TO PCL }\end{array}$ \\
\hline$P C_{L} O U T(8)$ & $\mathrm{PC}_{\mathrm{H}} \mathrm{OUT}$ & $\begin{array}{l}\text { LOWER ADD. } \\
\text { TO REG. }\end{array}$ & 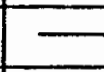 & $\longrightarrow$ & $P C_{L}$ OUT(8) & $\mathrm{PC}_{\mathrm{H}} \mathrm{OUT}$ & $\begin{array}{l}\text { HIGHEF ADD. } \\
\text { REG. A }\end{array}$ & $\begin{array}{l}\text { AEG. } \\
\text { TO } P C_{H}\end{array}$ & $\begin{array}{l}\text { REG.b } \\
\text { TO PCL }\end{array}$ \\
\hline$P C_{L}$ OUT(8) & $\mathrm{PC}_{\mathrm{H}} \mathrm{OUTT}$ & $\begin{array}{l}\text { LOWER ADO } \\
\text { TO REG.b }\end{array}$ & - & $\longrightarrow$ & $P C_{L}$ OUT/Bi & ${ }^{P C} C_{H}$ OUT & $\begin{array}{r}\text { HIGHER ADD } \\
\text { REG. } 8 \text { (12) }\end{array}$ & $\begin{array}{l}\text { REG. } \\
\text { TOPC }\end{array}$ & $\begin{array}{l}\text { REG. b } \\
\text { TOPC }\end{array}$ \\
\hline$P C_{L}$ OUT (8) & $\mathrm{PC}_{\mathrm{H}} \mathrm{OUT}$ & $\begin{array}{l}\text { LOWEA ADD. } \\
\text { TO REG. } \mathrm{b}\end{array}$ & & $\rightarrow$ & $P C_{L}$ OUTI(8) & $\mathrm{PC}_{\mathrm{HOUT}}$ & $\begin{array}{r}\text { HIGHER ADO } \\
\text { PEG. O (12) } \\
\end{array}$ & $\begin{array}{l}\text { REG. } \\
\text { TO } P C_{H}\end{array}$ & $\begin{array}{l}\text { REG.b } \\
\text { TO PCL }\end{array}$ \\
\hline & & & & & & & & & \\
\hline & & & & & & & & & \\
\hline & & & & & & & & & \\
\hline & & & & & & . & & & \\
\hline$\cdot$ & & & & & & & & & \\
\hline $\begin{array}{l}\text { REG. A } \\
\text { TO OUT (15) }\end{array}$ & $\begin{array}{l}\text { REG.b } \\
\text { TO OUT }\end{array}$ & $\begin{array}{l}\text { DATA TO } \\
\text { PEG. b }\end{array}$ & $\begin{array}{l}\text { COND } 11 \\
\text { OUT } 116 \\
\end{array}$ & $\begin{array}{l}\text { AEG.b } \\
\text { TO REG. A }\end{array}$ & & & 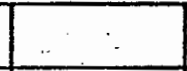 & & . \\
\hline $\begin{array}{l}\text { REG. A (15) } \\
\text { TO OUT }\end{array}$ & $\begin{array}{l}\text { REG. } \\
\text { TO OUT }\end{array}$ & $\begin{array}{c}x \\
(17) \\
\end{array}$ & & & & & & & \\
\hline
\end{tabular}

\begin{tabular}{|c|c|c|c|c|c|c|c|c|c|}
\hline & & & & & & $\cdots$ & & & \\
\hline \\
\hline
\end{tabular}

12. When the CALL is conditional and the condition fails, stotes T4 and T5 are skipped' and the state counter advances to the next memory cycle. If the condition is true, the stock is pushed of T4, and the lower and higher order addrews bytes are loaded into the program counter.

13. When the RETURN condition is true, pop up the suck: otherwise, advance to next mermory cycle skipping T4 and T6. 4. Bits $D_{3}$ through $D_{5}$ are loaded into $P C_{L}$ and all other bin are set to zero; zeros ore looded into $\mathrm{PC}_{\mathrm{H}}$.
15. PCC cycle (1/O Crcle).

16. The content of the condition flip-flops is avillable of the date but: $S$ at $D_{0}, Z$ at $D_{1}, P$ at $D_{2}, C$ at $D_{3}$.

17. A READY Command must be supplied for the OUT operation to be completed. An idle T3 state is used and then the state counter odvences to the next memory cycle.

18. When HALT commend occurs, the CPU internelly remains in the T3 state unsil an INTERRUPT in recognized. Externally. the STOPPED state is indiceted. 


\section{V.- PROCESSOR CONTROL SIGNALS}

\section{A. Interrupt Signal (INT)}

\section{1) INTERRUPT REQUEST}

If the interrupt line is enabled (Logic " 1 "), the CPU recognizes an interrupt request at the next instruction fetch (PCI) cycle by outputting $S_{0} S_{1} S_{2}=011$ at T1l time. The lower and higher order address bytes of the program counter are sent out, but the program counter is not advanced. A successive instruction fetch cycle can be used to insert an arbitrary instruction into the instruction register in the CPU. IIf a multi-cycle or multibyte instruction is inserted, an interrupt need only be inserted for the first cycle.)

When the processor is interrupted, the system INTERRUPT signal must be synchronized with the leading edge of the $\phi_{1}$ or $\phi_{2}$ clock. To assure proper operation of the system, the interrupt line to the CPU must not be allowed to change within $200 \mathrm{~ns}$ of the falling edge of $\phi_{1}$. An example of a synchronizing circuit is shown on the schematic for the SIM8-01 (Section VII).

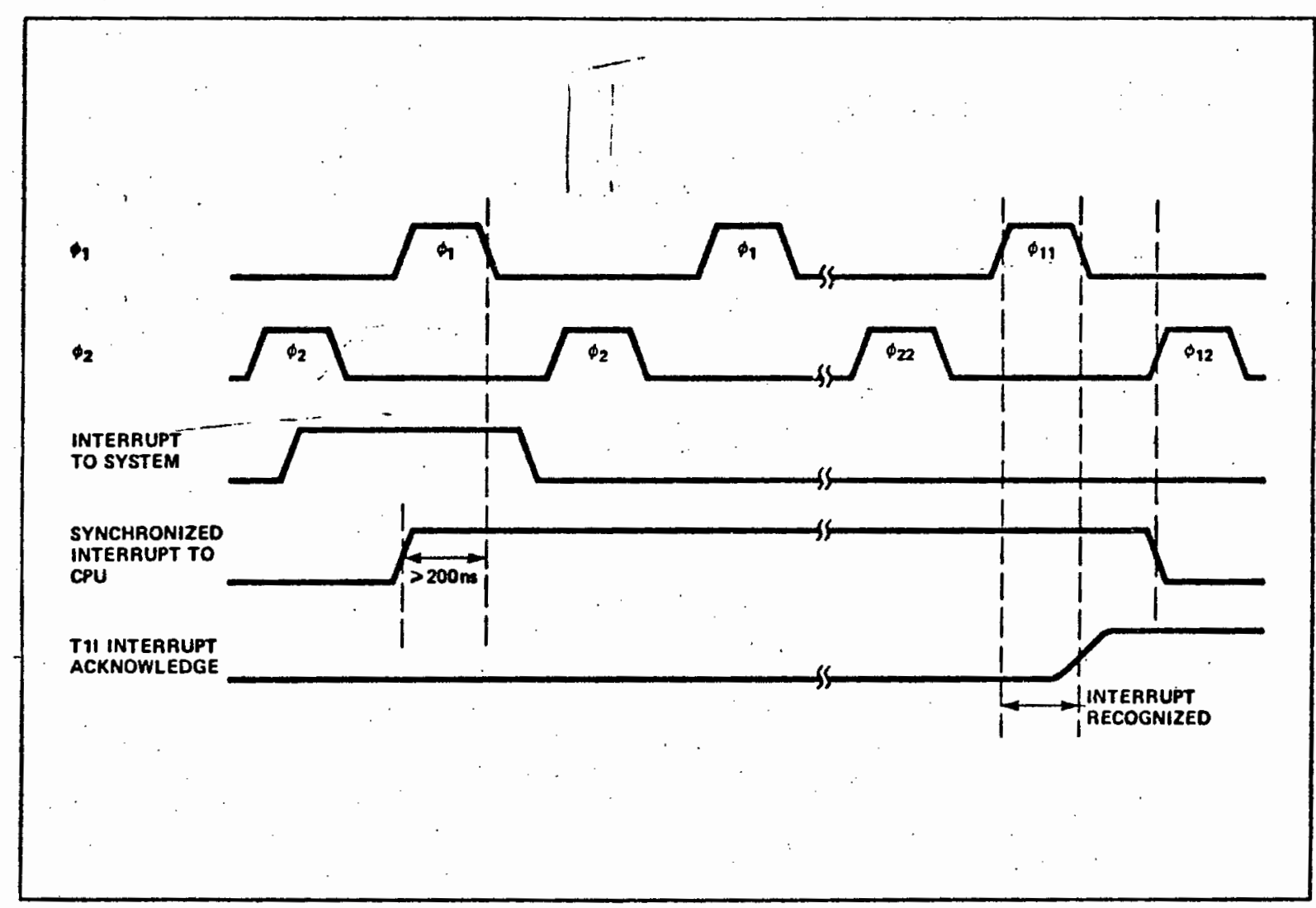

Figure 4. Recognition of Interrupt

If a HALT is inserted, the CPU enters a STOPPED state; if a NOP is inserted, the CPU continues; if a "JUMP to 0 " is inserted, the processor executes program from location 0 , etc. The RESTART instruction is particularly useful for handling interrupt routines since it is a one byte call. 


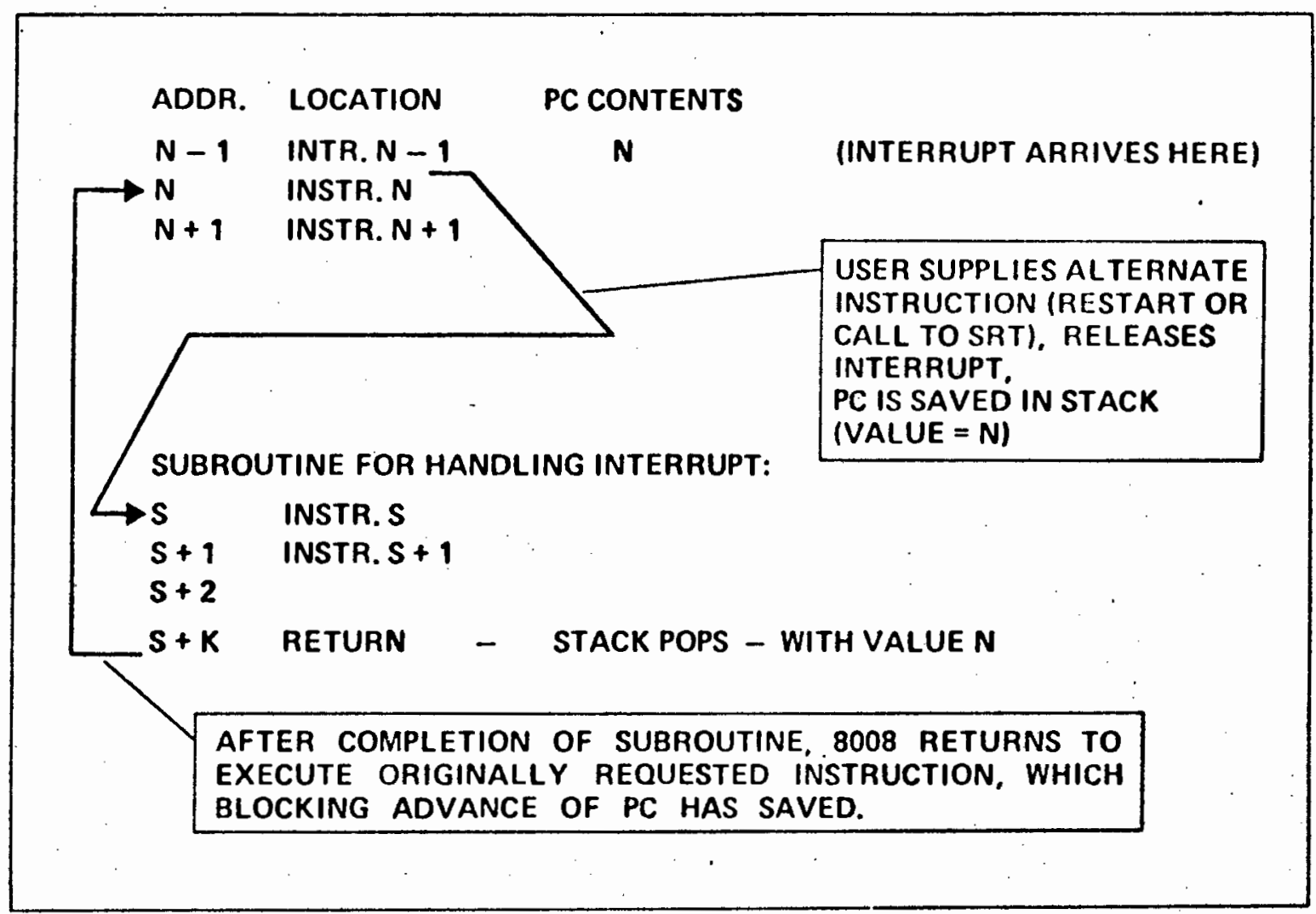

Figure 5. 8008 Interrupt

\section{2) START-UP OF THE 8008}

When power $\left(V_{D D}\right)$ and clocks $\left(\phi_{1}, \phi_{2}\right)$ are first turned on, a flip-flop internal to the 8008 is set by sensing the rise of $V_{D D}$. This internal signal forces a HALT $(00000000)$ into the instruction register and the 8008 is then in the STOPPED state. The following sixteen clock periods after entering the STOPPED state are required to clear (logic " 0 ") memories (accumulator, scratch pad, program counter, and stack). During this time the interrupt line has been at logic " 0 ". Any time after the memories are cleared, the 8008 is ready for normal operation.

To reset the flip-flop and also escape from the stopped state, the interrupt line must go tor a logic " $1 " ;$; It should be returned to logic " 0 " by decoding the state T1l at some time later than $\phi_{11}$. Note that whenever the 8008 is in a T1l state, the program counter is not incremented. As a result, the same address is sent out on two successive cycles.

Three possible sequences for starting the 8008 are shown on the following page. The RESTART instruction is effectively a one cycle call instruction, and it is convenient to use this instruction to call an initiation subroutine. Note that it is not necessary to start the 8008 with a RESTART instruction.

The selection of initiation technique to use depends on the sophistication of the system using the 8008. If the interrupt feature is used only for the start-up of the 8008 use the ROM directly, no additional external logic associated with instructions from source other than the ROM program need be considered. If the interrupt feature is used to jam instructions into the 8008 , it would then be consistent to use it to jam the initial instruction.

The timing for the interrupt with the start-up timing is shown on an accompanying sheet. The jamming of an instruction and the suppression of the program counter update are handled the same for all interrupts. 


\section{EXAMPLE 1:}

Shown below are two start-up alternatives where an instruction is not forced into the 8008 during the interrupt cycle. The normal program flow starts the 8008 .

a. 8008 ADDRESS OUT
00000000
0000000000
$\begin{array}{llllllllllll}0 & 0 & 0 & 0 & 0 & 0\end{array}$
$\begin{array}{lllllllll}0 & 0 & 0 & 0 & 0 & 0 & 0 & 0\end{array}$
$\begin{array}{llllll}0 & 0 & 0 & 0 & 0 & 0\end{array}$
$\begin{array}{lllllllll}0 & 0 & 0 & 0 & 0 & 0 & 0 & 1\end{array}$
$\begin{array}{llllll}0 & 0 & 0 & 0 & 0 & 0\end{array}$
$\begin{array}{llllllll}0 & 0 & 0 & 0 & 0 & 0 & 1 & 0\end{array}$

NOP
NOP
INSTR
INSTR
IN

b. 8008 ADDRESS OUT

\begin{tabular}{|c|c|c|}
\hline $\begin{array}{llllll}0 & 0 & 0 & 0 & 0 & 0 \\
0 & 0 & 0 & 0 & 0 & 0 \\
0 & 0 & 0 & 0 & 0 & 0\end{array}$ & $\begin{array}{llllllll}0 & 0 & 0 & 0 & 0 & 0 & 0 & 0 \\
0 & 0 & X & Y & Z & 0 & 0 & 0 \\
0 & 0 & X & Y & Z & 0 & 0 & 1\end{array}$ & $\begin{array}{l}\text { RST } \\
\text { INSTR }_{1} \\
\text { INSTR }_{2}\end{array}$ \\
\hline . & • & $\dot{.}$ \\
\hline
\end{tabular}

\section{INSTRUCTION IN ROM}

(LAA 11000000 ) Entry Directly To Main Program

\section{EXAMPLE 2:}

A RESTART instruction is jammed in and first instruction in ROM initially ignored.

\section{ADDRESS OUT}

$\begin{array}{llllllllllllll}0 & 0 & 0 & 0 & 0 & 0 & 0 & 0 & 0 & 0 & 0 & 0 & 0 & 0\end{array}$

$00000000 \quad 00 X Y Z 000$

$00000000000 \times Y Z O 001$

0000000.00

$\begin{array}{llllll}0 & 0 & 0 & 0 & 0 & 0\end{array}$

00000000 i 0 n n n n n $\begin{array}{lllllllllll}0 & 0 & 0 & 0 & 0 & 0 & 0 & 0\end{array}$ 0000000001 .

\section{INSTRUCTION IN ROM}

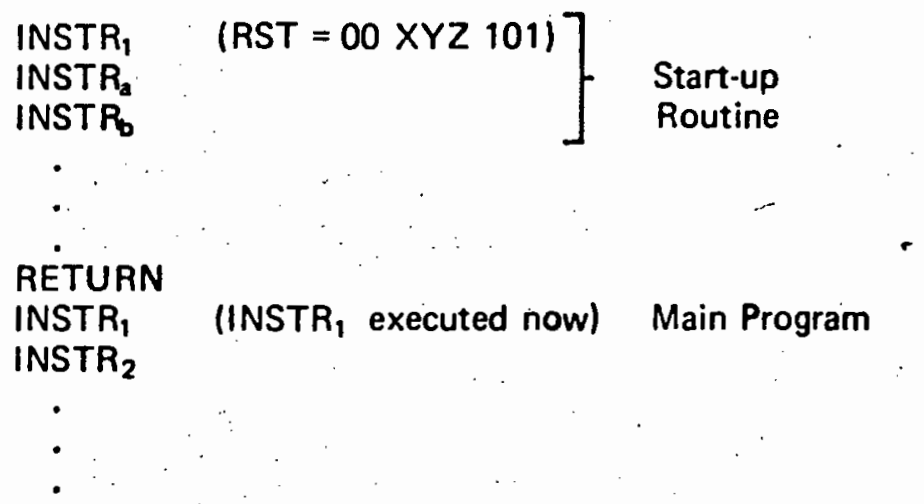

Note that during the interrupt cycle the flow of the instruction to the 8008 either from ROM or another source must be controlled by hardware external to 8008 .

\section{B. Ready (RDY)}

The 8008 is designed to operate with any type or speed of semiconductor memory. This flexibility is provided by the READY command line. A high-speed memory will always be ready with data (tie READY line to $V_{C C}$ / almost immediately after the second byte of the address has been sent out. As a result the 8008 will never be required to wait for the memory. On the other hand, with slow ROMs, RAMs or shift registers, the data will not be immediately available; the 8008 must wait until the READY command indicates that the valid memory data is available. As a result any type or any combination of memory types may be used. The READY command line synchronizes the 8008 to the memory cycle. When a program is being developed, the READY signal provides a means of stepping through the program, one cycle at a time. 


\section{ELECTRICAL SPECIFICATION}

The following pages provide the electrical characteristics for the 8008. All of the inputs are TTL compatible, but input pull-up resistors are recommended to insure proper $V_{1 H}$ levels. All outputs are low-power TTL compatible. The transfer of data to and from the data bus is controlled by the CPU. During both the WAIT and STOPPED states the data bus output buffers are disabled and the data bus is floating.

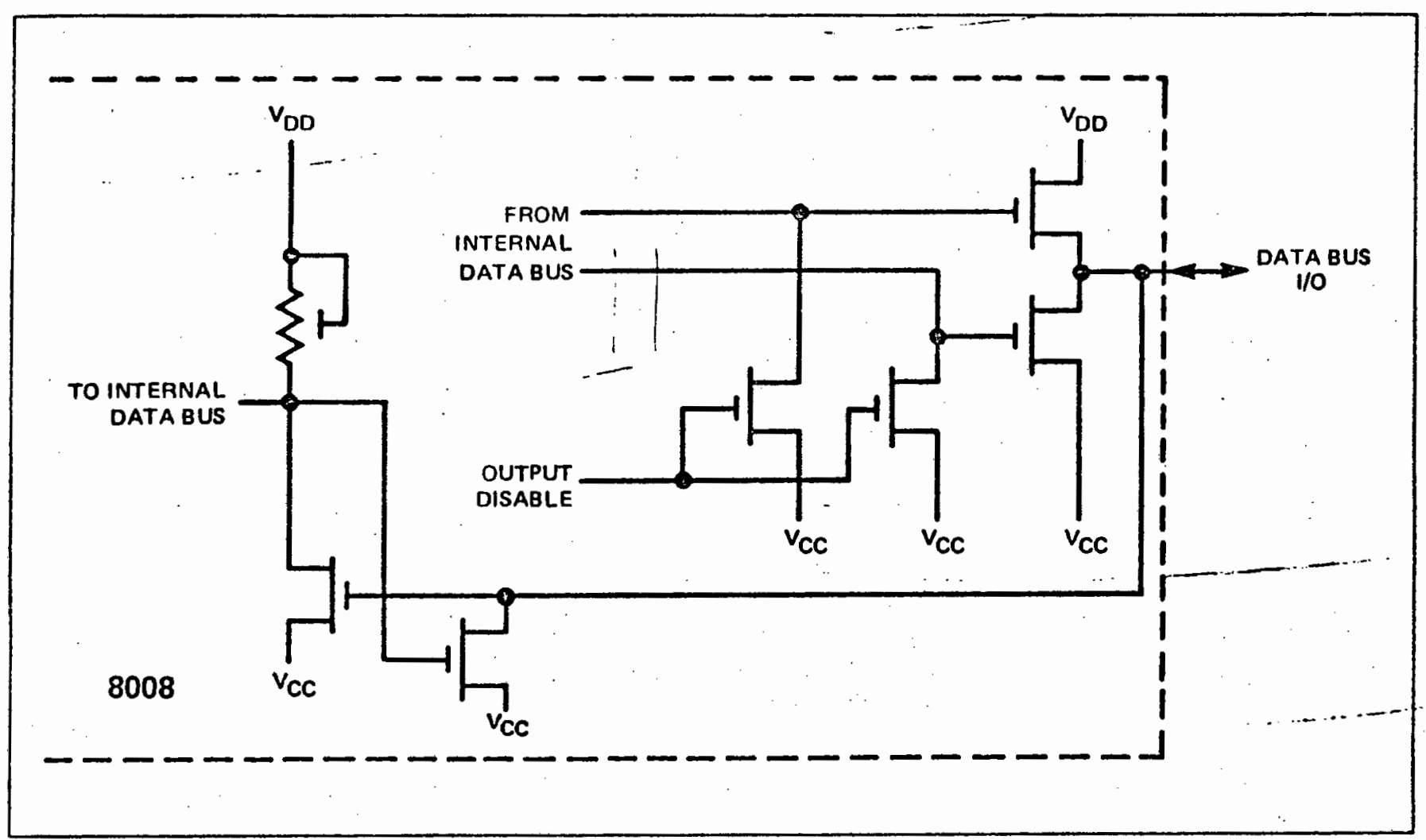

Figure 6. Data Bus 1/0 Buffer

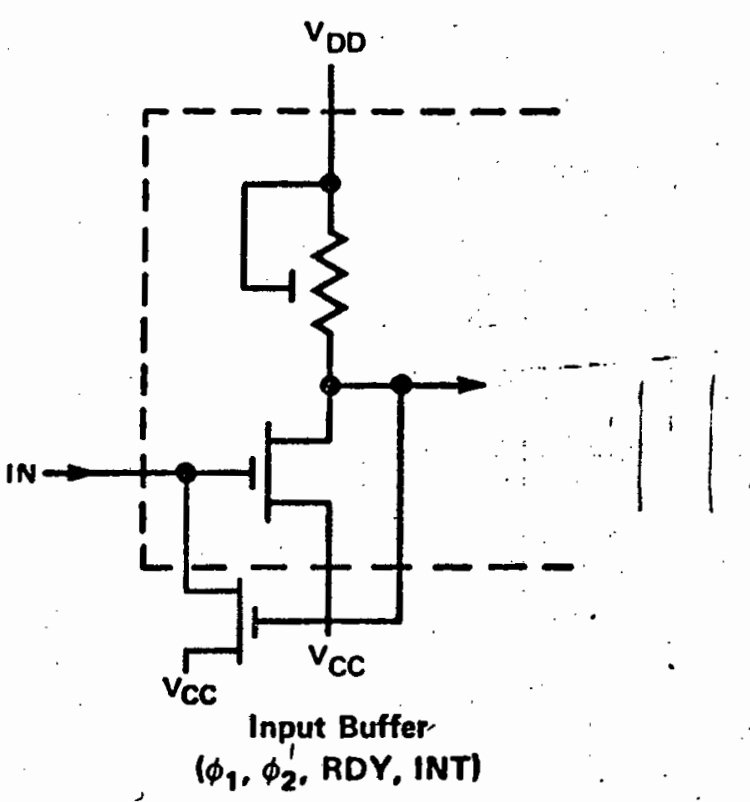

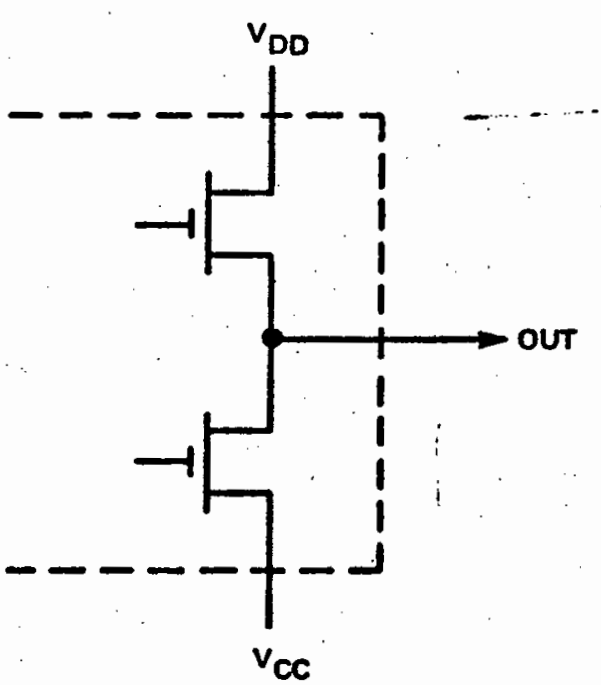

Outpat Buffer

(SYNC, $s_{0}, s_{1}, s_{2}$ )

Figure 7. 1/0 Circuitry 


\section{ABSOLUTE MAXIMUM RATINGS*}

\begin{tabular}{|c|c|c|}
\hline $\begin{array}{c}\text { Ambient Temperature } \\
\text { Under Bias }\end{array}$ & $0^{\circ} \mathrm{C}$ to $+70^{\circ} \mathrm{C}$ & 'COMMENT \\
\hline Storage Temperature & $-55^{\circ} \mathrm{C}$ to $+150^{\circ} \mathrm{C}$ & $\begin{array}{l}\text { Stresses above those listed under "Absolute Max- } \\
\text { imum Ratings" may cause permanent damage to }\end{array}$ \\
\hline $\begin{array}{l}\text { Input Voltages and Supply } \\
\text { Voltage With Respect } \\
\text { to } V_{\mathrm{CC}}\end{array}$ & +0.5 to $-20 \mathrm{~V}$ & $\begin{array}{l}\text { the device. This is a stress rating only and func- } \\
\text { tional operation of the device at these or any other } \\
\text { condition above those indicated in the operational }\end{array}$ \\
\hline Power Dissipation & $1.0 \mathrm{~W} @ 25^{\circ} \mathrm{C}$ & $f$ this specification is not implied. \\
\hline
\end{tabular}

\section{D.C. AND OPERATING CHARACTERISTICS}

$T_{A}=0^{\circ} \mathrm{C}$ to $70^{\circ} \mathrm{C}, V_{C C}=+5 V \pm 5 \%, V_{D D}=-9 V \pm 5 \%$ unless otherwise specified. Logic " 1 " is defined as the more positive tevel $\left(V_{1 H}, V_{O H}\right)$. Logic " 0 " is defined as the more negative level $\left(V_{1 L}, V_{O L}\right)$.

\begin{tabular}{|c|c|c|c|c|c|c|}
\hline \multirow{2}{*}{ SYMBOL } & \multirow{2}{*}{ PARAMETER } & \multicolumn{3}{|c|}{ LIMITS } & \multirow{2}{*}{ UNIT } & \multirow{2}{*}{$\begin{array}{c}\text { TEST } \\
\text { CONDITIONS }\end{array}$} \\
\hline & & MIN. & TYP. & MAX. & & \\
\hline$I_{D O}$ & $\begin{array}{l}\text { AVERAGE SUPPLY CURRENT- } \\
\text { OUTPUTS LOADED }\end{array}$ & & 30 & 60 & $m A$ & $T_{A}=25^{\circ} \mathrm{C}$ \\
\hline$I_{L I}$ & INPUT LEAKAGE CURRENT & & & 10 & $\mu \mathrm{A}$ & $v_{i N}=0 V$ \\
\hline$V_{1 L}$ & $\begin{array}{l}\text { INPUT LOW VOLTAGE } \\
\text { (INCLUDING CLOCKS) }\end{array}$ & $V_{D D}$ & & $V_{c c}-4.2$ & v & \\
\hline$V_{\text {IN }}$ & $\begin{array}{l}\text { INPUT HIGH VOLTAGE } \\
\text { (INCLUDING CLOCKS) }\end{array}$ & $v_{c c}-1.5$ & & $V_{c c}+0.3$ & v & \\
\hline $\mathrm{V}_{\mathrm{OL}}$ & OUTPUT LOW VOLTAGE & & & 0.4 & v & $\begin{array}{l}I_{Q L}=0.44 \mathrm{~mA} \\
C_{L}=200 \mathrm{pF}\end{array}$ \\
\hline $\mathrm{V}_{\mathrm{OH}}$ & OUTPUT HIGH VOLTAGE & $v_{c c}-1.5$ & & & v & $I_{\mathrm{OH}}=0.2 \mathrm{~mA}$ \\
\hline
\end{tabular}

- Measurements are made while the 8008 is executing a typical sequence of instructions. The test load is selected such that at $V_{O L}=0.4 \mathrm{~V} .1_{O L}=0.44 \mathrm{~mA}$ on each output.

\section{A.C. CHARACTERISTICS}

$T_{A}=0^{\circ} \mathrm{C}$ to $70^{\circ} \mathrm{C} ; V_{C C}=+5 \mathrm{~V} \pm 5 \%, V_{D D}=-9 \mathrm{~V} \pm 5 \%$. All measurements are referenced to $1.5 \mathrm{~V}$ levels.

\begin{tabular}{|c|c|c|c|c|c|c|c|}
\hline \multirow{3}{*}{ SYMBOL } & \multirow{3}{*}{ PARAMETER } & \multirow{2}{*}{\multicolumn{2}{|c|}{$\frac{8008}{\text { LIMITS }}$}} & \multirow{2}{*}{\multicolumn{2}{|c|}{ 8008-1 }} & \multirow{3}{*}{ UNIT } & \multirow{3}{*}{ TEST CONDITIONS } \\
\hline & & & & & & & \\
\hline & & MIN. & MAX. & MIN. & MAX. & & \\
\hline$t_{\mathrm{Cr}}$ & CLOCK PERIOD & 2 & 3 & 1.25 & 3 & $\mu s$ & $t_{R}, t_{F}=50 \mathrm{~ns}$ \\
\hline$t_{R}, t_{F}$ & CLOCK RISE AND FALL TIMES & & 50 & & 50 & ns & ? \\
\hline $\mathbf{t}_{\phi_{1}}$ & PULSE WIDTH OF $\phi_{1}$ & .70 & & .35 & & $\mu s$ & $\therefore$ \\
\hline${ }^{t} \phi 2$ & PULSE WIDTH OF $\phi_{2}$ & .55 & & .35 & & $\mu s$ & \\
\hline$t_{D 1}$ & $\begin{array}{l}\text { CLOCK DELAY FROM FALLING } \\
\text { EDGE OF } \phi_{1} \text { TO FALLING EDGE } \\
\text { OF } \phi_{2}\end{array}$ & .90 & 1.1 & & 1.1 & $\mu \mathbf{s}$ & $\vdots$ \\
\hline$t_{\mathrm{D} 2}$ & CLOCK DELAY FROM $\phi_{2}$ TO $\phi_{1}$ & .40 & & .35 & & $\mu \mathbf{s}$. & $\vdots$ \\
\hline$t_{D 3}$ & CLOCK DELAY FROM $\phi_{1}$ TO $\phi_{2}$ & .20 & & $.20-$ & 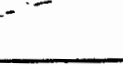 & $\mu s$ & : \\
\hline$t_{D D}$ & DATA OUT DELAY & & 1.0 & & 1.0 & $\mu \mathrm{s}$ & $C_{L}=100 \mathrm{pF}$ \\
\hline $\mathrm{t}_{\mathrm{OH}}$ & HOLD TIME FOR DATA BUS OUT & .10 & & .10 & & $\mu \mathrm{s}$ & \\
\hline$t_{1 H}$ & HOLD TIME FOR DATA IN & $|1|$ & & {$[1]$} & . & $\mu \mathrm{s}$ & \\
\hline $\mathrm{t}_{\mathrm{SD}}$ & SYNC OUT DELAY & & .70 & & .70 & $\mu s$ & $C_{L}=100 \mathrm{pF}$ \\
\hline$t_{s i}$ & $\begin{array}{l}\text { STATE OUT DELAY (ALL STATES } \\
\text { EXCEPT T } 1 \text { AND T } 11)^{(2)}\end{array}$ & & 1.1 & & 1.1 & $\mu s$ & $C_{L}=100 \mathrm{pF}$ \\
\hline$t_{s 2}$ & $\begin{array}{l}\text { STATE OUT DELAY (STATES } \\
\text { TI AND TII) }\end{array}$ & & .1 .0 & & 1.0 & $\mu s$ & $C_{\mathrm{L}}=100 \mathrm{pF}$ \\
\hline$t_{R W}$ & $\begin{array}{l}\text { PULSE WIDTH OF READY DURING } \\
\phi_{22} \text { TO ENTER T3 STATE }\end{array}$ & .35 & & .35 & & $\mu \mathrm{s}^{\circ}$ & 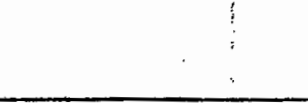 \\
\hline$t_{R D}$ & $\begin{array}{l}\text { READY DELAY TO ENTER WAIT } \\
\text { STATE }\end{array}$ & .20 & & .20 & & $\mu \mathrm{s}$ & \\
\hline
\end{tabular}

\footnotetext{
$[1]_{t_{1 H}} \mathrm{MIN} \geq t_{\text {SO }}$
}

$|2|$ If the INTERRUPT is not used, all states hafe the came output delay, isi. 
TIMING DIAGRAM

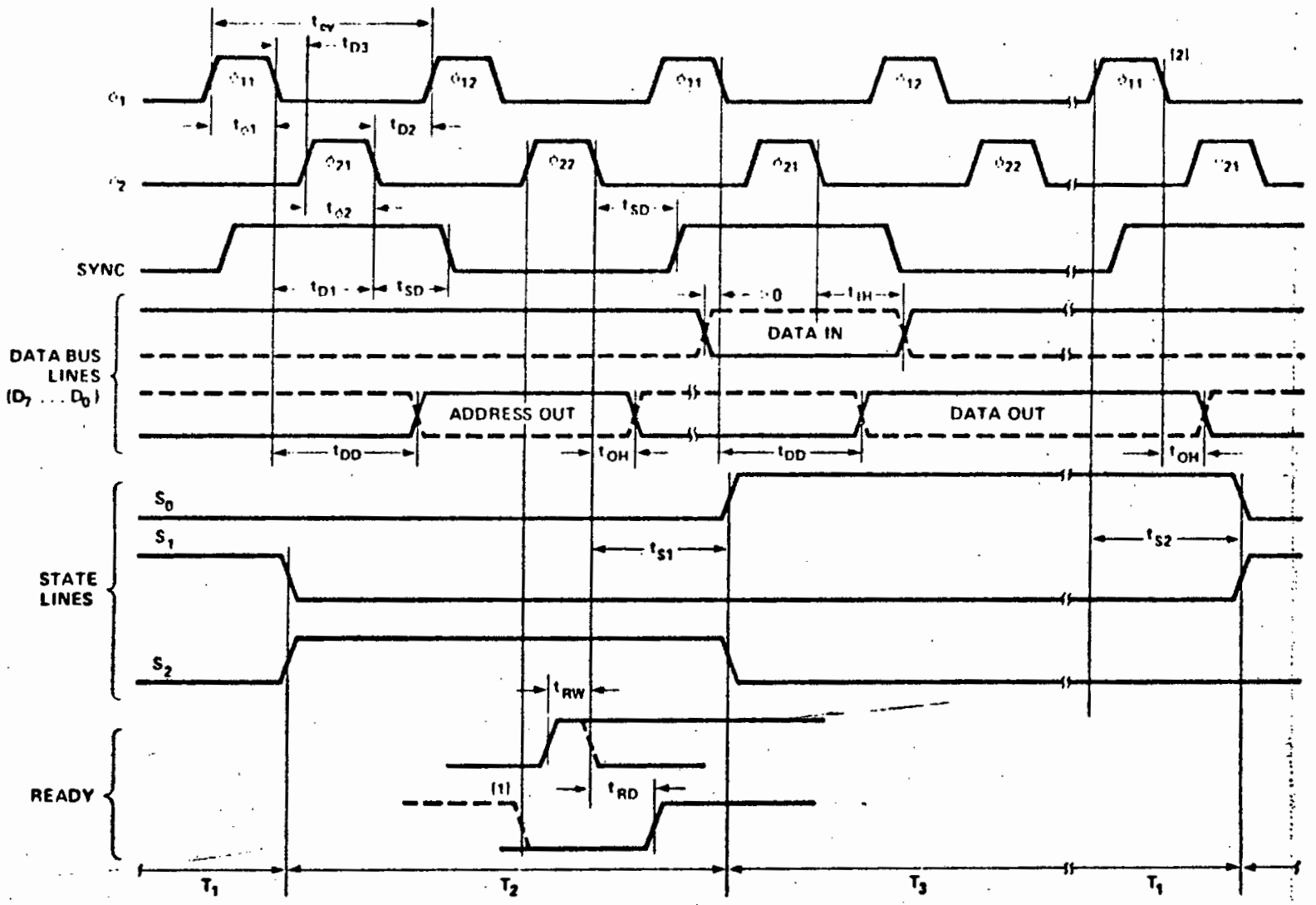

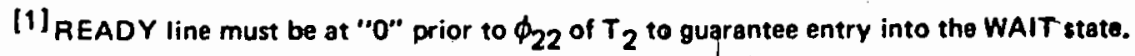

TYPICAL D.C. CHARACTERISTICS
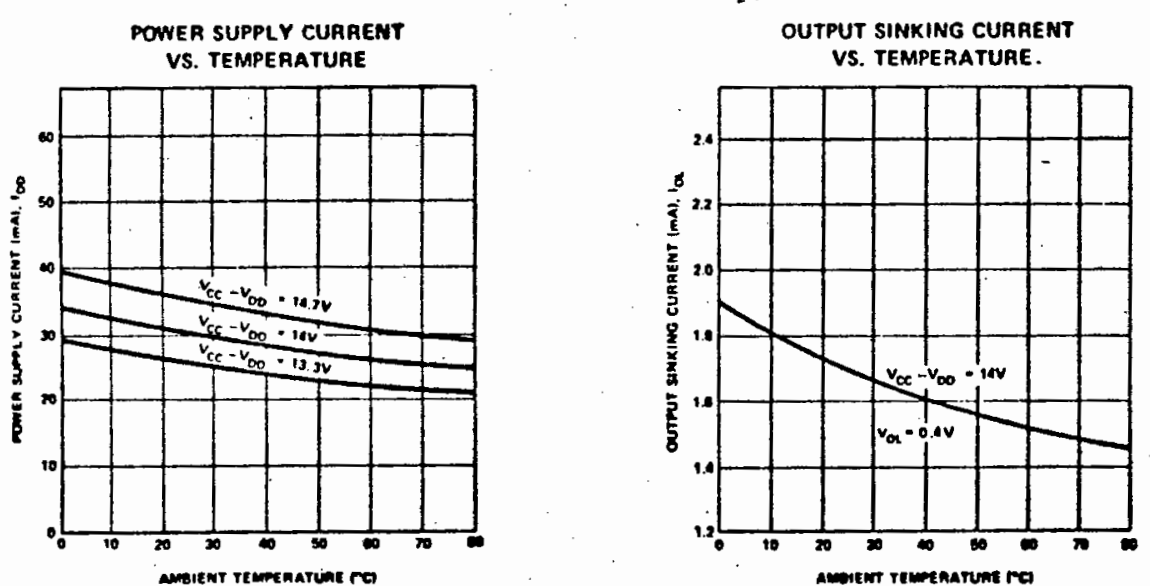

TYPICAL A.C. CHARACTERISTICS

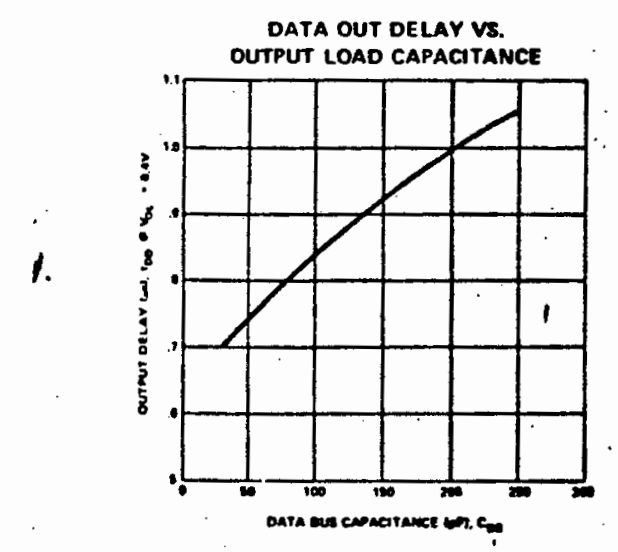

OUTPUT SOUREE CURRENT VS. OUTPUT VOLTAGE

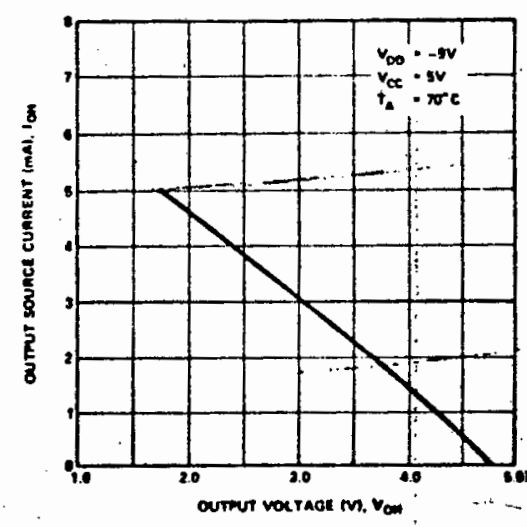

CAPACITANCE $f=1 \mathrm{MHz} ; T_{A}=25^{\circ} \mathrm{C}$; Unmeasured Pins Grounded.

\begin{tabular}{|c|c|c|c|}
\hline \multirow{2}{*}{ SYMBOL } & TEST & \multicolumn{2}{|c|}{ LIMUT (pF) } \\
\cline { 3 - 4 } & $\mathrm{C}_{\mathrm{IN}}$ & TYP. & MAX. \\
\hline $\mathrm{C}_{\mathrm{DB}}$ & INPUT CAPACITANCE & 5 & 10 \\
\hline $\mathrm{C}_{\mathrm{OUT}}$ & DATA BUS I/O CAPACITANCE & 5 & 10 \\
\hline
\end{tabular}




\section{BIT FULLY DECODED STATIC MOS RANDOM ACCESS MEMORY}

- Single +5 Volts Supply Voltage

- Directly TTL Compatible - All Inputs and Oulput

- Static MOS - No Clocks or Refreshing Required

- Low Power - Typically $150 \mathrm{~mW}$

- Access Time - Typically 500 nsec

- Three-State Output - OR-Tle Capability
- Simple Memory Expansion - Chip Enable input

- Fully Decoded - On Chip Address Decode

- Inputs Protected - All Inputs Have Protection Against Static Charge

- Low Cost Packaging - 16 Pin Plastic - Dual-In-Line Configuration

The Intel $\left.\right|^{\oplus} 2102$ is a 1024 word by one bit static random access memory element using normally off N-channel MOS devices integrated on a monolithic array. It uses fully DC stable (static) circuitry and therefore requires no clocks or refreshing to operate. The data is read out nondestructively and has the same polarity as the input data.

The 2102 is designed for memory applications where high performance, low cost, large bit storage, and simple interfacing are important design objectives.

It is directly $T T L$ compatible in all respects: inputs, output, and a single +5 volt supply. A separate chip enable $(\overline{C E})$ lead allows easy selection of an individual package when outputs are OR-tied.

The Intel 2102 is fabricated with $\mathrm{N}$-channel silicon gate technology. This technology allows the design and production of high performance easy to use MOS circuits and provides a higher functional density on a monolithic chip than either conventional MOS technology or P-channel silicon gate technology.

Intel's silicon gate technology also provides excellent protection against contamination. This permits the use of low cost silicone packaging.

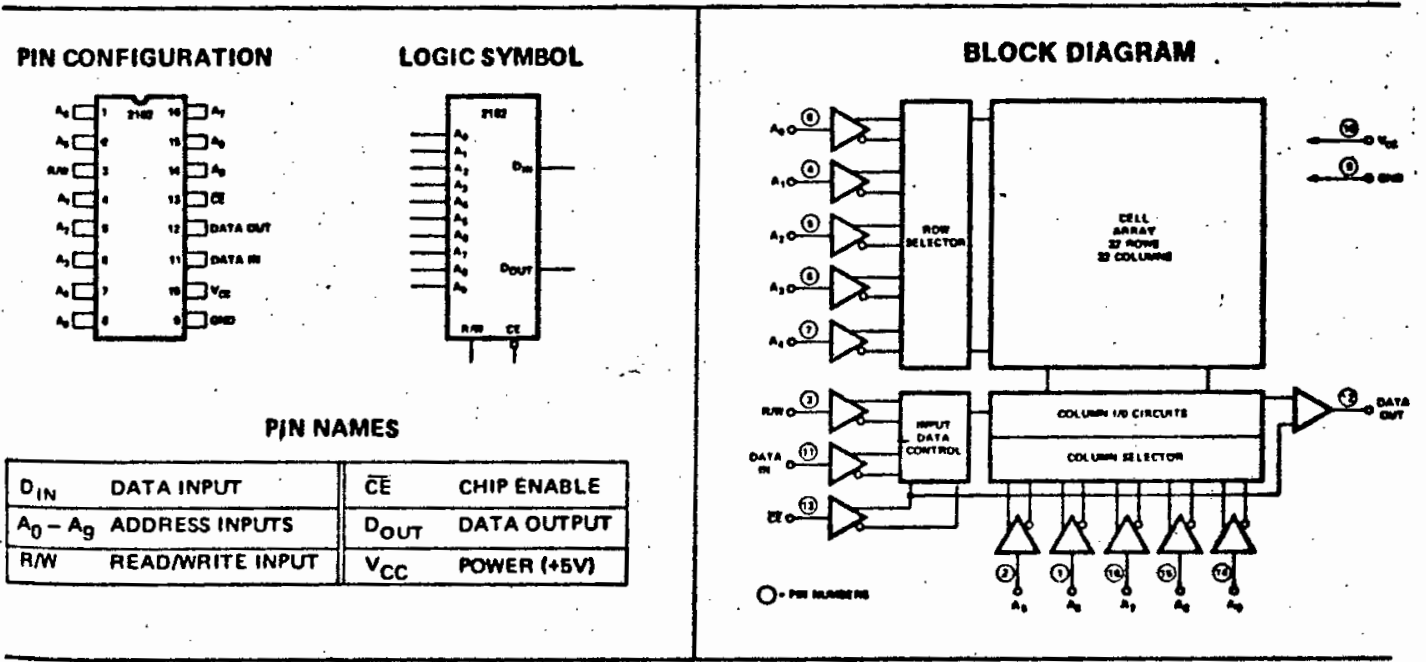


SILICON GATE MOS 2102

\section{Absolute Maximum Ratings*}

Ambient Temperature Under Bias

$0^{\circ} \mathrm{C}$ to $3 \mathrm{C}^{\circ} \mathrm{C}$

Storage Temperature

$-65^{\circ} \mathrm{C}$ to +150

Voltage On Any Pin

With Respect To Ground

Power Dissipation
$-0.5 V$ to +70

1 Wo:?

\section{EUAAMENT:}

St Si:n Pating" may cause permanent damage to the t.:. . This is a stress rating oniv and functional couraisun of the device at these or at any other compicion above those indicated in the operational secicions of this specification is not implied. Exposur to absoltte raximum rating conditions for extended periods may affect device reliability.

\section{C. and Operating Characteristlcs}

$T_{A}=0^{\circ} \mathrm{C}$ to $+70^{\circ} \mathrm{C}, V_{c C}=5 \mathrm{~V} \pm 5 \%$ unless otherwise specified

\begin{tabular}{|c|c|c|c|c|c|c|}
\hline \multirow{2}{*}{ SYMEOL } & \multirow{2}{*}{ PARAMETER } & \multicolumn{3}{|c|}{ LIMiTS } & \multirow{2}{*}{ UNIT } & \multirow{2}{*}{ TEST CONOITIONS } \\
\hline & & MUN. & TYP.11 & $\operatorname{MAX}$. & & \\
\hline$T_{L I}$ & $\begin{array}{l}\text { INPUT LOAD CURRENT } \\
\text { (ALL INPUT PINS) }\end{array}$ & & & 10 & $\mu \mathrm{A}$ & $V_{1 N}=0105.26 \mathrm{~V}$ \\
\hline ILOH & OUTPUT LEAKAGE CURRENT & & & 10 & $\mu \mathrm{A}$ & $\mathrm{CE}=2.2 \mathrm{~V}, \mathrm{~V}_{\mathrm{OUT}}=4.0 \mathrm{~V}$ \\
\hline ILOL & OUTPUT LEAKAGE CURRENT & & & -100 & $\mu A$ & $\overline{\mathrm{CE}}=2.2 \mathrm{~V}, \mathrm{~V}_{\mathrm{QUT}}=0.45 \mathrm{~V}$ \\
\hline Ioci & POWER SUPPLY CURAENT & & 30 & 60 & $m A$ & $\begin{array}{l}\text { ALLINPUTS }=5.25 \mathrm{~V} \\
\text { DATA OUT OPEN } \\
T_{A}=25^{\circ} \mathrm{C}\end{array}$ \\
\hline Tcc2 & POWER SUPPLY CURRENT & & & 70 & $m$ & $\begin{array}{l}\text { ALL INPUTS }=5.25 \mathrm{~V} \\
\text { DATA OUT OPEN } \\
T_{A}=0^{\circ} \mathrm{C}\end{array}$ \\
\hline$\overline{\nabla_{I L}}$ & INPUT "LOW" VOLTAGE & -0.5 & & +0.65 & $\bar{v}$ & 9 \\
\hline$V_{I H}$ & INPUT "HIGH" VOLTAGE & 2.2 & & $v_{C C}$ & $\bar{v}$ & \\
\hline$\frac{\text { in }}{V_{O L}}$ & OUTPUT "LOW" VOLTAGE & & & +0.45 & $\mathrm{~V}$ & $\mathrm{~T}_{\mathrm{OL}}=1.8 \mathrm{~mA}$ \\
\hline$V_{O H}$ & OUTPUT "HIGH" VOLTAGE & 2.2 & & & v & $T_{O H}=-100 \mu A$ \\
\hline
\end{tabular}

\section{Typical D.C. Characteristics}
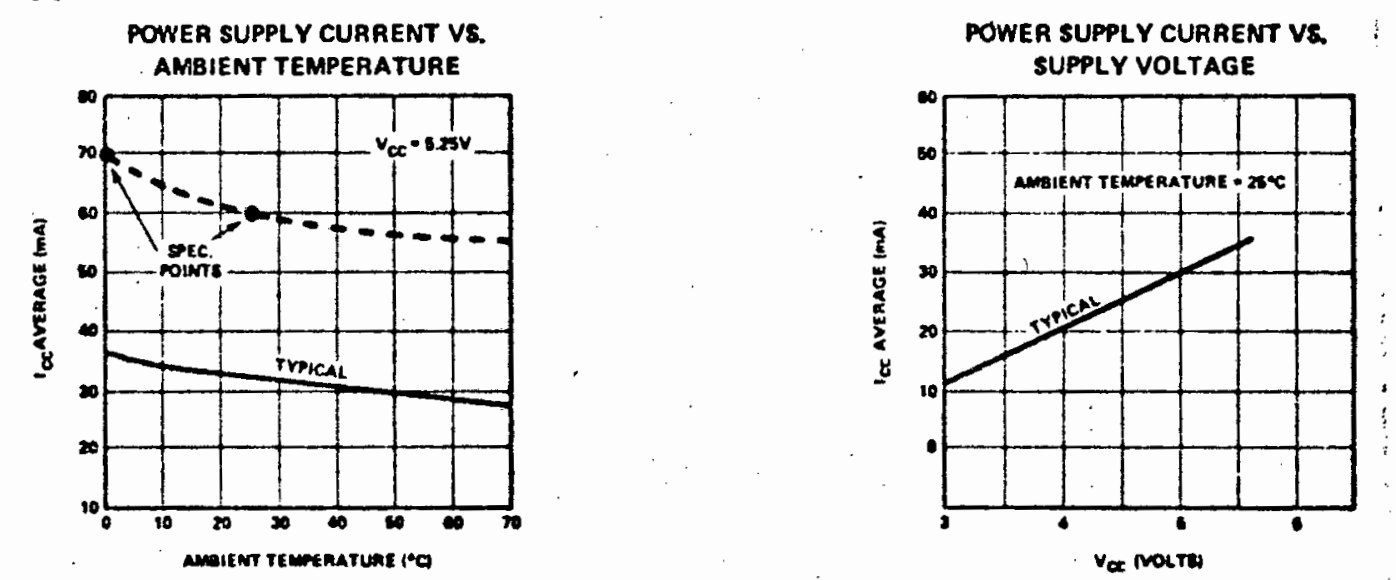

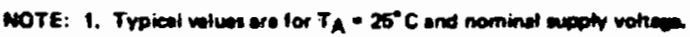


SILCON GATE MOS 2102

A. C. Characteristics $T_{A}=0^{\circ} \mathrm{C}$ to $70^{\circ} \mathrm{C}, V_{c C}=5 \mathrm{~V} \pm 5 \%$ unless otherwise specified

\begin{tabular}{|c|c|c|c|c|c|}
\hline \multirow{2}{*}{ SYMBOL } & \multirow{2}{*}{ PARAMETER } & \multicolumn{3}{|c|}{ LIMITS } & \multirow{2}{*}{ UNIT } \\
\hline & & MIN. & TYP. ${ }^{111}$ & $\max$ & \\
\hline \multicolumn{6}{|c|}{ READ CYCLE } \\
\hline$t_{R C}$ & READ CYCLE & 1000 & & & $m$ \\
\hline$t_{A}$ & ACCESS TIME & & 500 & 1000 & $n=$ \\
\hline$t_{\infty}$ & CHIP ENABLE TO OUTPUT TIME & & & 500 & $m$ \\
\hline tomi & $\begin{array}{l}\text { PREVIOUS READ DATA VALIO WITH RESPECT } \\
\text { TO ADDRESS }\end{array}$ & 50 & & & $n$ \\
\hline OH2 & $\begin{array}{l}\text { PREVIOUS READ DATA VALID WITH RESPECT } \\
\text { TO CHIP ENABLE }\end{array}$ & 0 & & & $m$ \\
\hline \multicolumn{6}{|c|}{ WRITE CYCLE } \\
\hline$i_{\text {we }}$ & WRITE CYCLE & 1000 & & & $m$ \\
\hline tAW & ADDRESS TO WRITE SETUP TIME & 200 & & & ns \\
\hline twp & WRITE PULSE WIDTH & 750 & & & $m$ \\
\hline$t_{\text {wh }}$ & WRITE RECOVERY TIME & 50 & & & ns \\
\hline$t_{\text {Dew }}$ & DATA SETUP TIME & 800 & & & $m$ \\
\hline $\mathrm{t}_{\mathrm{OH}}$ & DATA HOLD TIME & 100 & & & $m$ \\
\hline$t_{\mathrm{CW}}$ & CHIP ENABLE TO WRITE SETUP TIME & 900 & & & m \\
\hline
\end{tabular}

\section{A.C. CONDITIONS OF TEST}

\section{Inout Puive Lievols:}

Input Pulsa Rise and Fall Timos:

+0.65 Volt to 2.2 Volt

Timing Meourement Reference Lovel: $\quad 1.5 \mathrm{Volt}$

Output Load: $\quad 1 \pi L$ Gate and $C_{L}=100$ pF

\begin{tabular}{|c|c|c|c|}
\hline \multirow{2}{*}{ SYMBOL } & \multirow{2}{*}{ TEST } & \multicolumn{2}{|c|}{ LIMITS (pF) } \\
\hline & & TYP.(11) & MAX. \\
\hline$c_{\text {IN }}$ & $\begin{array}{l}\text { INPUT CAPACITANCE } \\
\text { (ALL INPUT PINS) } V_{\text {IN }}=\text { ON }\end{array}$ & 3 & 5 \\
\hline$c_{\text {out }}$ & $\begin{array}{l}\text { OUTPUT CAPACITANCE } \\
V_{\text {OUT }}=\text { OV }\end{array}$ & 7 & 10 \\
\hline
\end{tabular}

\section{Waveforms}

AEAD CrCLE

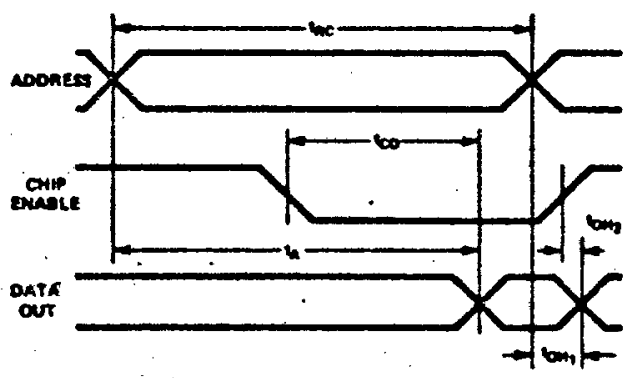

WAITE CYCLE

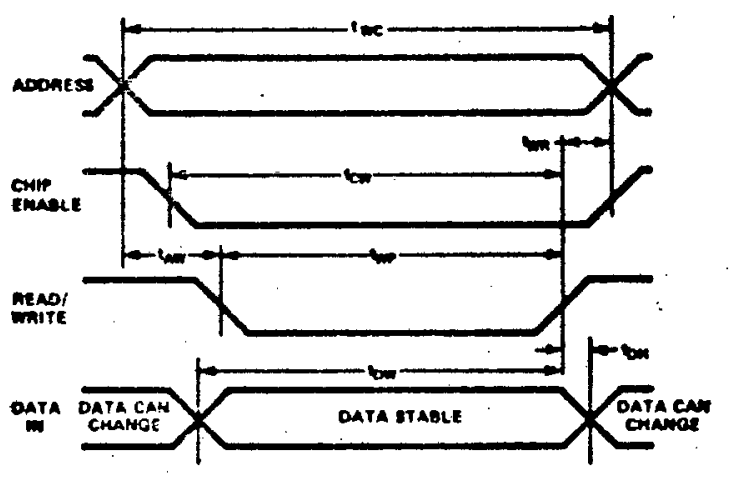

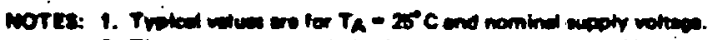

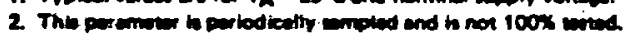




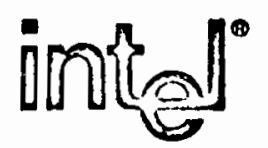

8212

EIGHT-BST. INPITT/OUTPUT PORT
The 8212 input/output port consists of an 8-bit latch with tri-state output buffers along with control and device selection logic. Also included is a service request flip-flop for the generation and control of interrupts to the microprocessor.

The device is multimode in nature. It can be used to implement latches, gated buffers or multiplexers. Thus, all of the principal.peripheral and input/output functions of a microcomputer system can be implemented with this device.

\section{Microcomputer Peripherals-- Schottky Bipolar}

- 3.65V Output High Voltage for Direct Interface to 8080 CPU or $8003 \mathrm{CPU}$

- Service Request Flip-Flop ifor Interrupt Generation

- Low Input Load Current $.25 \mathrm{~mA}$ Max.

- Three State Outputs

- Outputs Sink 15 mA
- Asynchronous Register Clear

- Replaces Buffers, Latches and Multiplexers in Microcomputer Systems

- Reduces System Package Count

PIN NAMES

\begin{tabular}{|l|l|}
\hline$D I_{1} \cdot D_{8}$ & DATA IN \\
\hline$D O_{1}-D O_{8}$ & DATA OUT \\
\hline$\overline{D S_{1}} \cdot D S_{2}$ & DEVICE SELECT \\
\hline$M D$ & MODE \\
\hline STB & STROBE \\
\hline$\overline{I N T}$ & INTERRUPT (ACTIVE LOW) \\
\hline$\overline{C L R}$ & CLEAR (ACTIVE LOW) \\
\hline
\end{tabular}

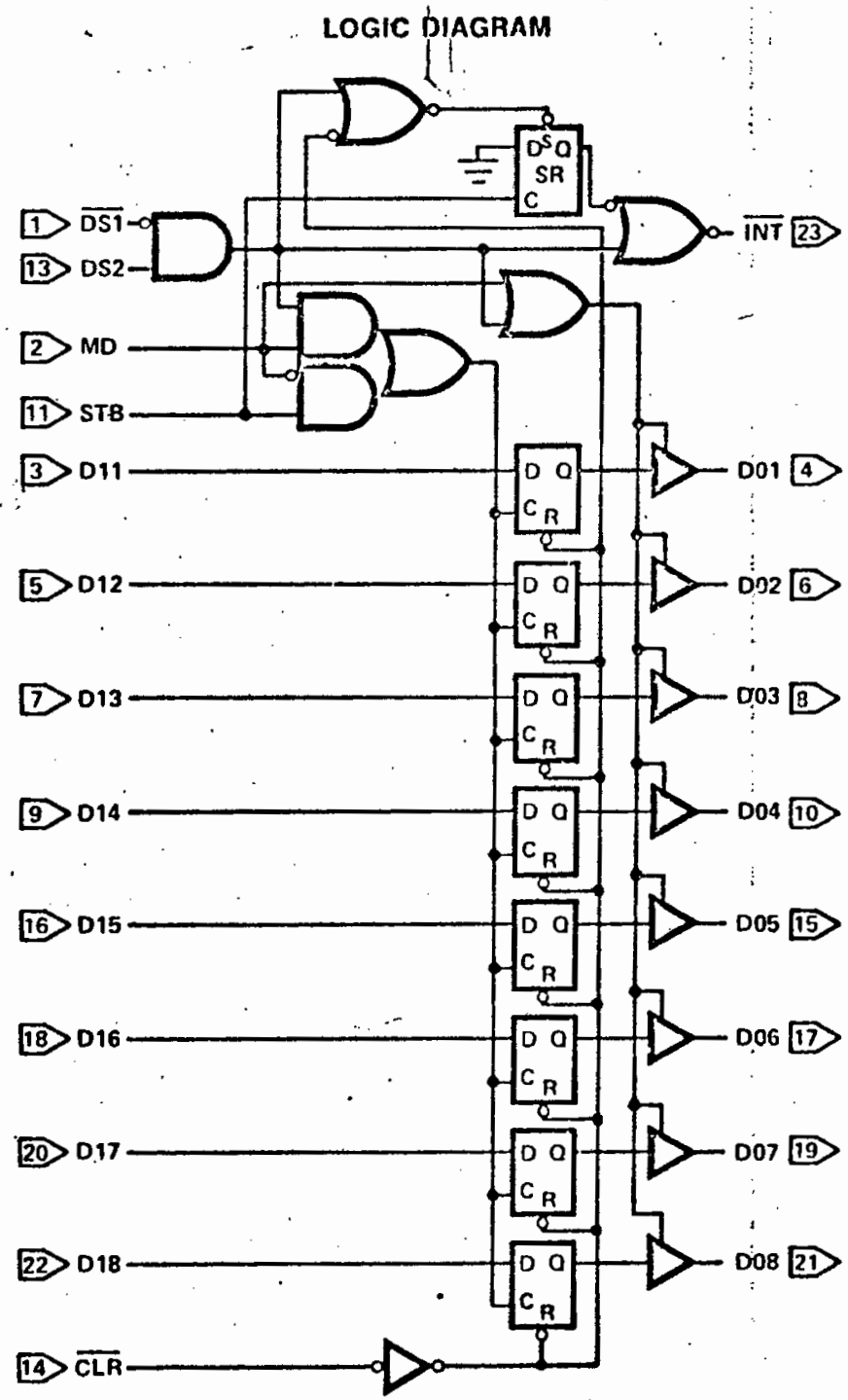


APPENDIX 5 - WITBANK SYSTEM PARAMETERS

Shown below.is a table giving the relevant constants of the weighing system used at present at Witbank.

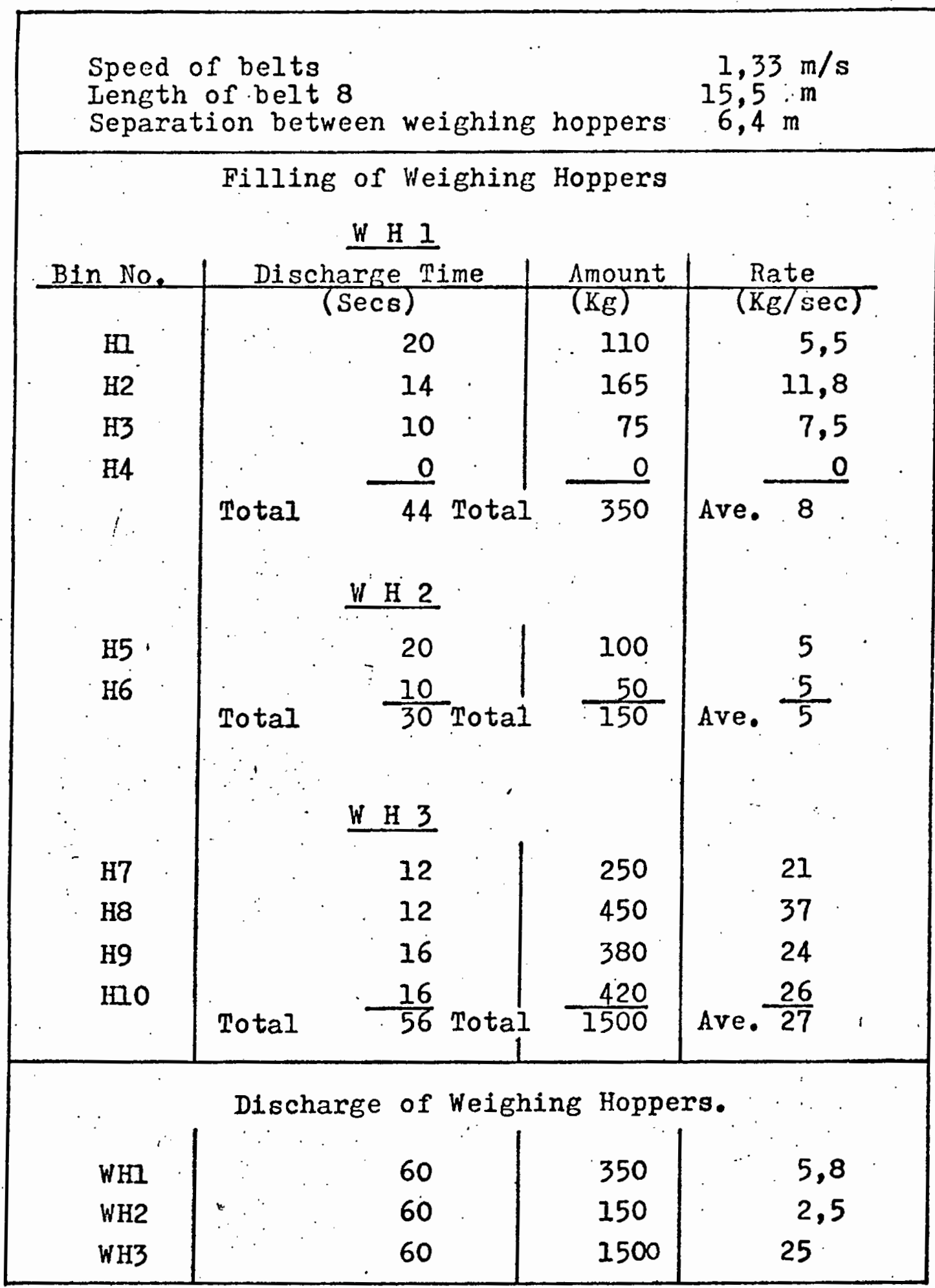

